\title{
MINOS Sterile Neutrino search
}

by

\author{
David Jason Koskinen
}

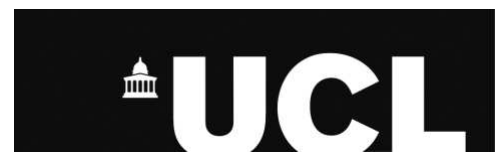

In partial fulfillment of the requirements for the degree of Doctor of Philosophy.

[February 2009] 


\section{Declaration}

I, David Jason Koskinen, confirm that the work presented in this thesis is my own. Where information has been derived from other sources, I confirm that this has been indicated in the thesis.

The original efforts and work contained in this thesis span three separate subjects: sterile neutrino formalism, work pertaining to the resolution of the muon flux from the NuMI beam and the Far/Near extrapolation method. Constructing a neutrino model containing a sterile neutrino was done in concert with the MINOS Neutral Current Convenor Brian Rebel and theorist Stephen Parke whilst at Fermilab. 


\section{Abstract}

The Main Injector Neutrino Oscillation Search (MINOS) is a long-baseline accelerator neutrino experiment designed to measure properties of neutrino oscillation. Using a high intensity muon neutrino beam, produced by the Neutrinos at Main Injector (NuMI) complex at Fermilab, MINOS makes two measurements of neutrino interactions. The first measurement is made using the Near Detector situated at Fermilab and the second is made using the Far Detector located in the Soudan Underground laboratory in northern Minnesota. The primary goal of MINOS is to verify, and measure the properties of, neutrino oscillation between the two detectors using the $v_{\mu} \rightarrow v_{\tau}$ transition. A complementary measurement can be made to search for the existence of sterile neutrinos; an oft theorized, but experimentally unvalidated particle.

The following thesis will show the results of a sterile neutrino search using MINOS RunI and RunII data totaling $\sim 2.5 \times 10^{20}$ protons on target. Due to the theoretical nature of sterile neutrinos, complete formalism that covers transition probabilities for the three known active states with the addition of a sterile state is also presented. 


\section{Acknowledgements}

I would like to thank Jenny for useful advice on my studies and a critical eye on this thesis. I am grateful to friends and office mates at UCL: Dan, Tom, Shiva and especially Mark and Leo for lively (non-physics) conversation as well as pints at the pub. Mark Lancaster has singularly saved my financial future from certain ruin in wading through the UCL bureaucracy on my behalf.

Whilst at Fermilab Caius Howcroft, Peter Shanahan and Bob Zwaska have become good friends and saved my sanity. Eric Grashorn has been kind enough to share his wine, cooking and friendship. Mandy and David Rominsky are good people. Phil Adamson was instrumental in easing my transition to Fermilab. Alex Sousa, Robert Pittam and the rest of the NC working group have been consistently helpful and gracious colleagues. Sacha Kopp has taught me things that I never thought I would need to learn, and I am all the more grateful for it. Instrumental to this thesis and my work on MINOS has been the considerable help and sagacious advice from Brian Rebel, who was also kind enough to join me on many bike rides along the Fox river.

My family, from Manuela Forte to the Portillas, grandma and grandpa, cousins and on to the Roberts, have been instrumental in providing a bedrock of support during my studies. Ben, Greg and Sherm have been great friends. I thank my wife for being continually supportive and understanding when either studies or work kept us apart.

Lastly, I would like to express my unbounded gratitude to my mother. 


\section{Contents}

\begin{tabular}{llr}
\hline Introduction & 15
\end{tabular}

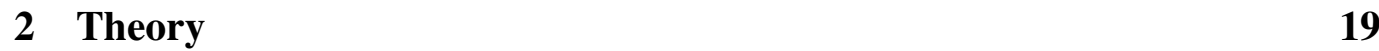

2.1 Three neutrinos . . . . . . . . . . . . . . . . . . 19

2.2 Oscillation Theory $\ldots \ldots \ldots 22$

2.2 .1 Three neutrino model . . . . . . . . . . . . 23

2.2 .2 Neutrino Oscillations in Matter - MSW . . . . . . . . . 27

2.2 .3 Oscillation including a sterile neutrino . . . . . . . . 30

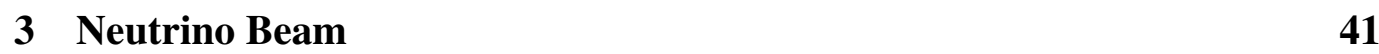

3.1 Protons . . . . . . . . . . . . . . . . . . . . 41

$3.1 .1 \mathrm{H}^{-}$source/Cockroft-Walton . . . . . . . . . . 42

3.1 .2 Linac . . . . . . . . . . . . . . . . 44

3.1 .3 Booster . . . . . . . . . . . . . . . 45

3.1 .4 Main Injector . . . . . . . . . . . . . . . . . . . . 47

3.2 NuMI Beamline $\ldots \ldots \ldots$. . . . . . . . . . . . . . . 49

3.3 Hadron reweighting - SKZPTV . . . . . . . . . . . . . . . 53

3.4 Muon Monitors . . . . . . . . . . . . . . . . . . . . . . 57

\begin{tabular}{|lll}
\hline 4 & The Detectors & 66
\end{tabular}

$4.1 \quad$ Collective properties $\ldots \ldots \ldots 6$

4.2 Calibration $\ldots \ldots \ldots \ldots$ 
$4.2 .1 \quad$ Light Injection $\ldots \ldots \ldots$. . . . . . . . . . . 70

4.2 .2 Cosmic Rays . . . . . . . . . . . . . . . 71

$4.2 .3 \quad$ Mapper . . . . . . . . . . . . . . 72

4.3 Magnet $\ldots \ldots \ldots \ldots . \ldots \ldots$

4.4 Near Detector . . . . . . . . . . . . . . . . 76

$4.4 .1 \quad$ Near Detector Electronics $\ldots \ldots \ldots 77$

4.5 Far Detector . . . . . . . . . . . . . . . . . . . . . . 79

$4.5 .1 \quad$ Far Detector Electronics $\ldots \ldots \ldots$. . . . . . . 80

4.6 Calibration Detector . . . . . . . . . . . . . . . . . . . 82

4.6 .1 The CalDet setup . . . . . . . . . . . . . . 82

5 Event Selection, Far/Near Extrapolation and Systematic Uncertainties 84

5.1 Event Reconstruction . . . . . . . . . . . . . . . . . . 85

5.1 .1 CandStrip . . . . . . . . . . . . . 86

5.1 .2 CandSlice . . . . . . . . . . . . . . . 87

5.1 .3 CandCluster $\ldots \ldots \ldots \ldots . \ldots . \ldots 8$

5.1 .4 CandShower . . . . . . . . . . . . . . 88

5.1 .5 CandEvent . . . . . . . . . . . . . . . 89

5.2 Near Detector Event Selection $\ldots \ldots \ldots$

$5.2 .1 \quad$ Split Events Pathology . . . . . . . . . . . . . . . 91

$5.2 .2 \quad$ Vertexing Failure Pathology $\ldots \ldots \ldots$

5.3 Far Detector Event Selection $\ldots \ldots . \ldots 95$

$5.3 .1 \quad$ Non-neutrino Pathologies $\ldots \ldots \ldots$. . . . . . . 95

$5.3 .2 \quad$ Particle Pathology $\ldots \ldots \ldots . \ldots 98$

5.4 Neutral-current/Charged-current Separation . . . . . . . . . . . 101

5.5 Neutral-current Extrapolation to Far Detector $\ldots$. . . . . . . . . 105

$5.5 .1 \quad$ Far Over Near Extrapolation . . . . . . . . . . . . . 106

5.5 .2 Optimization . . . . . . . . . . . . . 110 
5.6 Systematic Uncertainties . . . . . . . . . . . . . . . . . 110

5.6 .1 Neutrino-Nucleus Interaction. . . . . . . . . . . . . . 111

5.6 .2 Beam Modeling. . . . . . . . . . . . . . . . 112

5.6 .3 Hadronic Energy . . . . . . . . . . . . . . . . . . 112

5.6 .4 Normalization . . . . . . . . . . . . . . . . . . 114

$5.6 .5 \quad$ Charged-Current Background in the Neutral-Current Sample . . . . . . . . . . . . . . . 115

5.6 .6 Event Selection . . . . . . . . . . . . . . . . 116

5.6 .7 Electron Appearance Background $\ldots \ldots \ldots$. . . . . . 118

$\begin{array}{lll}6 & \text { Neutral-current Analysis } & 121\end{array}$

6.1 3Flavor Model . . . . . . . . . . . . . . . . . . . . . . . . . . 122

6.1 .1 Initial F/N Validation . . . . . . . . . . . . . . . . 122

6.1 .2 Systematic Uncertainty Shifts . . . . . . . . . . . . 125

6.2 4Flavor Model . . . . . . . . . . . . . . . . . . . . . . . . 131

$6.2 .1 \quad$ F/N Validation using 4Flavor Model . . . . . . . . . . . 132

6.2 .2 Systematic Uncertainty Shifts . . . . . . . . . . . . 134

6.3 Data Results . . . . . . . . . . . . . . . . . . . . . . . . . . 134

$6.3 .1 \quad 3$ Flavor . . . . . . . . . . . . . . . . . 134

6.3 .2 4Flavor . . . . . . . . . . . . . . . . 138

$\begin{array}{lll}7 & \text { Conclusion } & 142\end{array}$

\begin{tabular}{|l|l|}
\hline A Constraining the 3Flavor model probabilities & 145
\end{tabular}

\begin{tabular}{lll}
\hline B & F/N Systematic Effects & 151
\end{tabular} 


\section{List of Figures}

$2.1 \quad Z^{0}$ boson width from the combined results of the four LEP experi-

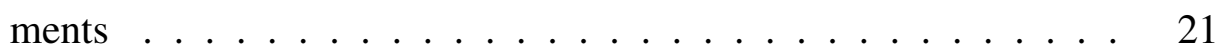

2.23 flavor neutrino hierarchy $\ldots \ldots \ldots$. . . . . . . . . . . 24

$2.3 \quad$ Survival probabilities in vacuo of $v_{\mu} \rightarrow v_{\mu}$ for three different values

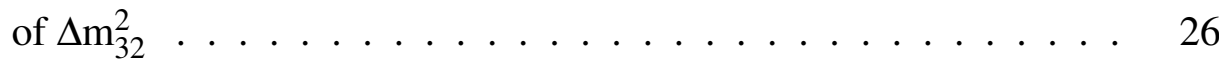

2.4 Feynman diagrams of the charge current forward scattering of the electrons with both electron neutrinos and antin-neutrinos. . . . . 28

2.5 Oscillation probabilities for $v_{\mu} \rightarrow v_{e} \ldots \ldots \ldots 29$

$3.1 \quad$ Magnetron plasma source . . . . . . . . . . . . . . . . 43

3.2 Linac to Booster Orbump focusing magnets and path . . . . . . . 45

3.3 Cartoon of slip-stacking in the Main Injector . . . . . . . . . . . . 48

$3.4 \quad$ NuMI beam path after extraction from the Main Injector $\ldots . . .50$

$3.5 \quad$ Monte Carlo energy spectrum of muon neutrino charged-current events in the Near Detector for three different beam configurations. 50

$3.6 \quad$ Neutrino production and focusing from the NuMI target through the magnetic horns, decay pipe, Hadron absorber and muon monitors 51

3.7 Monte Carlo visualization of a Muon Monitor with the spacing and size of the ionization cells $\ldots \ldots \ldots \ldots$. . . . . . . . . 52

3.8 SKZPTV weights as a function of $p_{t}$ vs. $p_{z} \ldots \ldots \ldots 5$ 
3.9 Near Detector charged-current energy spectrum for the RunII data set before and after applying SKZPTV weights $\ldots . . . . .56$

3.10 Feynman diagram of a pion decay producing a muon and muon neutrino in a one-to-one relationship . . . . . . . . . . . 57

3.11 Photo of the nine streamer tubes that make up each muon monitor 58

3.12 Location of each muon monitor in the NuMI beam . . . . . . . 59

3.13 Grid of that separates the end of the decay pipe into 6 regions . . . 60

3.14 Efficiency curves for a muon to reach the three muon monitors . . 61

3.15 Histogram of the muon momentum at the end of the decay pipe for the LE000z010i target position . . . . . . . . . . . . . . 62

3.16 Monte Carlo predicted spectrum of muons at the first muon monitor 63

3.17 Monte Carlo predicted signal compared to data . . . . . . . . . . 64

4.1 Connection made by the optical fibers for the detectors . . . . . 68

4.2 Cross section of scintillator module maniforld . . . . . . . . . . . 69

4.3 Drift response for the Near and Far detector . . . . . . . . . . . 72

4.4 Magnetic field map . . . . . . . . . . . . . . . . . . . . . . 74

4.5 Hysterisis looping showing the orientation of the magnetic domains during magnetic field reversal . . . . . . . . . . . . . 75

4.6 Scintillator plane layout . . . . . . . . . . . . . . . . . 77

$4.7 \quad$ Response of the different QIE chip ranges . . . . . . . . . . . . 78

4.8 Picture supermodule 2 of the Far Detector . . . . . . . . . . . . 80

4.9 Picture of the MINOS mux box $\ldots \ldots \ldots$. . . . . . . . 81

4.10 Picture of the Calibration detector located in the CERN PS . . . . 83

$5.1 \quad$ Flow diagram of the MINOS reconstruction $\ldots \ldots$. . . . . . . . 86

5.2 Fiducial volume of the Near Detector. . . . . . . . . . . . . . . . 90

$5.3 \quad$ NC energy spectrum of the Near Detector before application of the cleaning cuts to remove reconstruction pathologies $\ldots \ldots$. . . . 91 
$5.4 \quad$ NC energy spectrum in the Near Detector after the cleaning cuts are applied . . . . . . . . . . . . . . . 92

5.5 Time difference in between reconstructed events . . . . . . . . . . 93

5.6 Number of neutrino events in the FD after timing, fiducial and preselection cuts . . . . . . . . . . . . . . 100

5.7 Event display in both the U and V view of 3 Monte Carlo events . 102

5.8 Near Detector distributions of the three NC/CC separation vari-

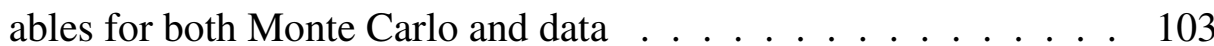

5.9 RunI and RunII ND data after all cuts as well as NC/CC event separation . . . . . . . . . . . . . . . . 104

5.10 Two histograms showing the relationship between true and reconstructed energy for the MINOS Monte Carlo data set in the Near Detector . . . . . . . . . . . . . . . . 108

$5.11 \mathrm{~F} / \mathrm{N}$ ratio for the extrapolation of the NC events from the Near Detector to the Far Detector . . . . . . . . . . . . . . . . . . 109

5.12 Muon neutrino cross-section calculations from the NEUGEN-V3 neutrino event generator $\ldots \ldots \ldots \ldots 111$

5.13 Ratio plots used for assessing the charged-current $v_{\mu}$ background using the alternate horn current method $\ldots \ldots$. . . . . . . 117

5.14 Histogram of the efficiency of classifying a true $\mathrm{v}_{e}$ charged-current event as a neutral-current event as a function of true neutrino energy 119

5.15 Charged-current spectra of the $v_{e}$ and $v_{\tau}$ backgrounds . . . . . 119

6.1 Mass squared splitting of the three active neutrinos as respective amounts of mass eigenstates in each flavor eigenstate . . . . . . . 122

6.2 Flow diagram of two methods used to test the Far/Near extrapolation method . . . . . . . . . . . . . . . . . . . 123 
6.3 Monte Carlo predictions of the charged-current and neutral-current energy spectrum using the standard 3Flavor model $\ldots . . . .124$

6.4 Results of the validation test for the Far/Near extrapolation . . . . 126

6.5 Histogram of the change in number of events in the $\mathrm{F} / \mathrm{N}$ predicted spectrum when the absolute hadronic energy is shifted by 11\% . . 128

6.6 Mass squared splitting and mass eigenstate location for the 4Flavor Parke model of neutrino oscillation . . . . . . . . . . . . . . . 131

6.7 Predicted CC and NC energy spectrum results of a Monte Carlo test using the 4Flavor model without systematic uncertainty shifts using two different data sets $\ldots \ldots \ldots . \ldots . \ldots 133$

6.8 3Flavor neutral-current and charged-current energy spectrum for the combined RunI and RunII data set with a Far Detector exposure of $2.46 \times 10^{20}$ POT, $\theta_{13}=0$ and $\delta_{C P}=0 \ldots \ldots \ldots \ldots$

6.9 3Flavor neutral-current and charged-current energy spectrum for the combined RunI and RunII data set with a Far Detector exposure of $2.46 \times 10^{20}$ POT, $\theta_{13}=0.21$ and $\delta_{C P}=3 \pi / 2 \ldots \ldots \ldots . . . . .137$

6.10 4Flavor neutral-current and charged-current energy spectrum for the combined RunI and RunII data set . . . . . . . . . . . . . . 139

6.11 Contour plots, 1D projections and fit values of the 4Flavor model for the oscillation parameters $\left|U_{\mu 3}\right|^{2}$ and $\left|U_{s 3}\right|^{2}$. The 1D projec\begin{tabular}{|c|c|}
\hline tions are computed by fixing the corresponding oscillation param- \\
\hline
\end{tabular} eters to their best fit value, i.e. the $\left|U_{\mu 3}\right|^{2}$ plot uses $\left|U_{s 3}\right|^{2}=0.11$ or 0.19 . The vertical abscissa on the 1D plots are in units of $\Delta \chi^{2}$. The dashed lines in the contour plot represent the $68 \%$ confidence interval while the solid lines represents the $90 \%$ interval. . . . . . 141

A.1 3Flavor transition probabilities for the set I inputs from table $\mid$ A.1 and the probability is constrained to be in the range $0-1 \ldots$. . . 146 
A.2 3Flavor transition probabilities for the set I inputs from table $\mid$ A.1 and the probability is unconstrained. . . . . . . . . . . . . . 147

A.3 3Flavor transition probabilities for the set II inputs from table A.1 and the probability is constrained to be in the range $0-1$. . . . . . 148

A.4 3Flavor transition probabilities for the set III inputs from table A.1 and the probability is constrained to be in the range $0-1$. . . . . . 149

A.5 3Flavor transition probabilities for the set IV inputs from table A.1 and the probability is constrained to be in the range $0-1 \ldots$. . . . 150

B.1 Energy spectrum shift caused by the changing the absolute hadronic calibration uncertainty by $\pm 1 \sigma$.

B.2 Energy spectrum shift caused by the changing the CC background uncertainty by $\pm 1 \sigma . \ldots \ldots \ldots$. . . . . . . . . . . 152

B.3 Energy spectrum shift caused by the changing the CCMA uncertainty by $\pm 1 \sigma . \ldots \ldots \ldots \ldots$. . . . . . . . . . 153

B.4 Energy spectrum shift caused by the changing the uncertainty on

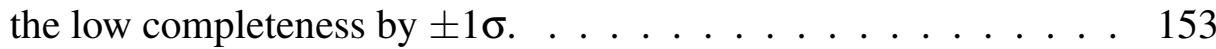

B.5 Energy spectrum shift caused by the changing the normalization uncertainty by $\pm 1 \sigma . \ldots \ldots \ldots$. . . . . . . . . . 154

B.6 Energy spectrum shift caused by the changing the relative hadronic calibration uncertainty by $\pm 1 \sigma . \ldots$. . . . . . . . . . . . . 154

B.7 Energy spectrum shift caused by the changing the uncertainty SKZP beam reweighting by $\pm 1 \sigma . \ldots \ldots \ldots \ldots$ 


\section{List of Tables}

2.1 Current best fit values for neutrino oscillation . . . . . . . . . . 30

5.1 Systematic errors on the absolute energy scale . . . . . . . . . . . 113

5.2 Event number uncertainty stemming from the event selection criteria 118

5.3 Values used in calculating the effect of $v_{e}$ appearance . . . . . . 119

$6.1 \Delta \mathrm{m}_{31}^{2}, \Delta \mathrm{m}_{32}^{2}$, and $\theta_{23}$ results for the validations tests of the Far/Near extrapolation method . . . . . . . . . . . . . . 125

6.2 Shifts in the oscillation parameters from the systematics in the 3Flavor model . . . . . . . . . . . . . . . . . . . . 128

6.3 The uncertaintity on the number of events in the Far Detector neutralcurrent spectrum for each systematic . . . . . . . . . . . . . 130

6.4 Shift in oscillation values from the two different methods of validating the Far/Near extrapolation method with the 4Flavor model . 134

6.5 Shifts in the oscillation parameters from the individual systematics

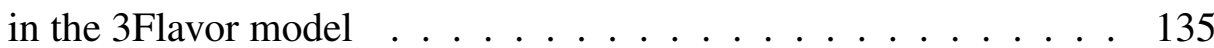

6.6 Number of events and level of agreement between data and F/N neutral-current prediction for the two MINOS sensitive energy regions . . . . . . . . . . . . . . . . 138 
A.1 Input parameters for the 3Flavor model. All values are best fit results from reactor, atmospheric and accelerator neutrino experiments, except for $\delta_{C P}$ which has no constraint between $0-2 \pi$. . . . 145 


\section{Chapter 1}

\section{Introduction}

The Standard Model has been one of the most successful theories ever created. It has elegantly combined Quantum Electrodynamics, Quantum Chromodynamics as well as the Weak force. Along with their antiparticles, the Standard Model (SM) contains 6 quarks, 6 leptons and the gauge bosons that mediate the electromagnetic, weak and strong force and has stood up to experimental verification at a level more precise than any other theory of what was known and has provided a template for the existence of new particles. While remaining a robust theory, the SM is incomplete in describing certain aspects of particle phenomena, such as Dark Matter, Dark Energy and neutrino masses to name a few. Specifically important to this thesis is that neutrino oscillations exist outside the Standard Model.

The neutrino was first proposed as a particle in 1930 by Wolfgang Pauli[1] in an effort to explain the electron energy spectrum anomaly in nuclear beta decay. The neutrino, as initially proposed, was an additional particle involved in the nuclear decay that had no electric charge, and a mass no greater than $1 \%$ the mass of the proton. While postulated in 1930, due to the low mass, small cross-section, zero charge and lack of direct detection via a decay chain, the neutrino waited until the mid 1950's for experimental verification.

In 1953, Frederick Reines and Clyde Cowan began work to prove the existence 
of the neutrino. Their experiment used liquid scintillator to record the production of three gamma rays from an electron anti-neutrino and proton interaction[2]. The positron produced in the interaction, $\overline{\mathrm{v}}_{e}+p \rightarrow n+e^{+}$, pair annihilates with an atomic electron to produce back-to-back gamma rays. The liquid scintillator was doped with cadmium to absorb the remnant neutron and produce an additional gamma ray $5 \mu \mathrm{sec}$ after the initial interaction. Because the dominant background to the neutrino signature was gamma ray production from cosmic ray muons, by either radiation, decay etc... the experiment required a high neutrino flux to amplify the signal and detector shielding to reduce the cosmic ray background.

The initial experiment was constructed near the Hanford nuclear reactor, which produced a neutrino flux of $10^{12}-10^{13}$ per square centimeter per second. The experiment was located on the surface of the earth and surrounded by 4-8 inches of lead shielding in combination with 4-6 feet of paraffin. It aimed to measure a rate difference in the three gamma ray signal when the reactor was operational versus dormant. The rate difference between on/off was within predicted errors and the result was consistent with no excess from neutrinos[3]. While the initial result was inconclusive, moving the detector from its surface location at the Hanford reactor to a buried location at the Savannah River nuclear plant produced convincing results that constituted verification[4]].

Later experiments in neutrino physics were once again predicated on nuclear reactions producing a large neutrino flux. The sun, being the largest available nuclear reactor, was utilized by John Bahcall and Ray Davis Jr. in a solar neutrino experiment started in 1961. The experiment used 100,000 gallons of cleaning fluid, tetrachlorethylene $\left(\mathrm{C}_{2} \mathrm{Cl}_{4}\right)$, located 4,850 feet underground in the Homestake Mine in South Dakota to detect solar neutrinos. The signature was radioactive ${ }_{18}^{37} \mathrm{Ar}$ produced from the interaction $\mathrm{v}_{e}+{ }_{17}^{37} \mathrm{Cl} \rightarrow e^{-}+{ }_{18}^{37} \mathrm{Ar}$. While the experiment worked in providing a sufficient signal to background ratio, a peculiarity appeared in the 
expected number of solar neutrinos; there existed a deficit of neutrinos compared to predictions using the standard solar model [5]. Later experiments using radiochemical detection, such as GALLEX [6] and SAGE[7], found a similar deficit in the solar neutrino rate. Other experiments measuring atmospheric neutrino rates, such as IMB [8], MACRO [9, 10], Soudan-2 [11, 12], and Kamiokande[13] also recorded a deficit in comparison to prediction.

The explanation of the neutrino deficit anomaly is a theory of leptonic flavor nonconservation originally proposed by Bruno Pontecorvo[ [14, 15]. The theory proposes that neutrinos are created and detected as weak, or flavor eigenstates, but propagate as a superposition of mass eigenstates. The difference in mass between the mass eigenstates would cause an oscillation between the flavors and explain the deficit anomaly; instead of neutrinos disappearing, they are instead oscillating to a flavor for which the experiment lacks sensitivity. The theory was expanded by Maki, Nakagawa and Sakata to incorporate the three known neutrino flavors muon, tau and electron[16] and successfully explains both the solar neutrino deficit[17 18, 19], and the atmospheric deficit[20].

New Physics in regard to neutrinos potentially extends beyond oscillations to the possibility of a sterile neutrino; a neutrino with no coupling to either the $\mathrm{W}^{ \pm}$ or $\mathrm{Z}^{0}$. The prospect of a sterile neutrino could provide one possible solution to various astrophysical puzzles such as cosmological nucleosynthesis[21].

The results from the Liquid Scintillator Neutrino Detector (LSND)[22] appeared to give a first to hint at the existence of sterile neutrinos in order to explain anomolous results for the $\overline{\mathrm{v}}_{\mu} \rightarrow \overline{\mathrm{v}}_{e}$ transition. The oscillation parameters measured from an excess of $\bar{v}_{e}$ stood in contrast to the results from the well measured atmospheric and solar oscillation parameters. The addition of a $4^{\text {th }}$ flavor of neutrino (sterile) went a long way towards rectifying the incongruence, but fell out of favor when the MiniBooNE experiment saw no similar excess[23, 24, 25]. In addition to 
the MiniBooNE refutation, a global fit to the LSND, MiniBooNE and other shortbaseline (SBL) experiments disfavors a 3 active plus 1 sterile neutrino scheme at the $4 \sigma$ level[26]. SBL experiments though are not the only means to test the existence of a sterile neutrino. While the parameter space available for a sterile neutrino is severely restricted from previous SBL and solar measurements, long-baseline accelerator (LBL) experiments probe a region of $v_{\text {active }} \rightarrow v_{\text {sterile }}$ parameter space to which previous experiments have been insensitive.

Because the three active neutrinos couple to the $Z^{0}$, the rate of neutrino neutralcurrent (NC) events should be unaffected by the standard 3 flavor neutrino oscillation. Conversely, existence of a sterile neutrino adds the possibility of a $v_{\text {active }} \rightarrow$ $v_{\text {sterile }}$ transition that would create a deficit in the rate of NC events. Neutrino oscillation experiments using different baselines, energies and initial neutrino flavors that measure the $\mathrm{NC}$ rate can in concert search for, or limit, the parameter space available for a sterile neutrino by looking for a deficit.

Using solar neutrinos, SNO has made a neutral-current rate measurement that favors $v_{e}$ to only $v_{\text {active }}$ at the $5.3 \sigma$ level[27] versus $v_{e}$ to only $v_{\text {sterile. }}$ In the case where the solar neutrinos can oscillate to both $v_{\text {active }}$ and $v_{s}$ the sterile amount is bound at a less than stringent $45 \%$ at the $99 \%$ C.L. [28, 29]. Super-K has similarly made a solar NC rate measurement that heavily favors pure $\mathrm{v}_{e} \rightarrow \mathrm{v}_{\text {active }}$ versus pure $v_{e} \rightarrow v_{s}$, but does allow for a small admixture of $v_{e}$ to $v_{s}$ as well as $v_{\text {active }}[30$, 31].

The following thesis contains such a LBL sterile neutrino search. The theory of neutrino oscillation and the perturbation of accommodating a sterile neutrino is covered in Chapter 2, the experimental apparatus is detailed in Chapters 3 and 4 and results of the search are detailed in Chapter 6 . 


\section{Chapter 2}

\section{Theory}

\subsection{Three neutrinos}

The discovery of the tau neutrino proved to be the last particle described by the Standard Model (SM)[32]. Seeing that a neutrino was the last discovered of the SM predicted fundamental particles, the question naturally arises, "If the SM predicts three neutrinos what is the experimental evidence that there are only three?" While many experiments probing the nature of neutrinos use a neutrino beam over a baseline, the number of active light neutrinos can be determined by measurement of the $Z$ boson width at collider experiments. The most complete set of such measurements to determine the number of neutrinos was conducted at CERN by the LEP experiments.

The Large Electron Positron (LEP) collider at CERN was home to four different detectors measuring the results of $e^{+} e^{-}$collisions: ALEPH, DELPHI, L3 and OPAL. From these collisions, measurements were made of the partial widths from the $Z^{0}$ decay, whose resonance follows a relativistic Breit-Wigner probability

distribution. Inherent in the decay products of the $Z^{0}$ is an invisible width, $\Gamma_{i n v}$, that is postulated to be the result of unobserved neutrinos. The invisible width can be expressed as the partial widths of the corresponding quarks and charged leptons 
subtracted from the $Z^{0}$ width $\left(\Gamma_{Z^{0}}\right)$ :

$$
\Gamma_{i n v}=\Gamma_{Z^{0}}-\left(\Gamma_{e e}+\Gamma_{\mu \mu}+\Gamma_{\tau \tau}+\Gamma_{h a d}\right),
$$

where $\Gamma_{i n v}$ is the invisible partial width, $\Gamma_{e e}$ the electron partial width, $\Gamma_{\mu \mu}$ the muon partial width, $\Gamma_{\tau \tau}$ the tau partial width and $\Gamma_{\text {had }}$ the hadronic partial width. Assuming lepton universality so that the branching ratios are the same for the three different flavors of lepton $\Gamma_{e e}+\Gamma_{\mu \mu}+\Gamma_{\tau \tau}=3 \Gamma_{l l}$, the above expression reduces to

$$
\Gamma_{i n v}=\Gamma_{Z^{0}}-3 \Gamma_{\ell \ell}-\Gamma_{h a d}
$$

The following set of equations give the ratio of the invisible partial width to the leptonic partial width in terms of measurable quantities,

$$
\begin{aligned}
R_{i n v}^{0} & \equiv \frac{\Gamma_{i n v}}{\Gamma_{\ell l}}, \\
& =\frac{\Gamma_{Z^{0}}-3 \Gamma_{l l}-\Gamma_{h a d}}{\Gamma_{\ell l}}, \\
& =\frac{\Gamma_{Z^{0}}-\Gamma_{h a d}}{\Gamma_{\ell l}}-3 .
\end{aligned}
$$

Using the expression for the hadronic pole cross-section ${ }^{1}$ below, the value $R_{i n v}^{0}$ can be written in terms of experimentally measured values[34].

$$
\begin{aligned}
\sigma_{\text {had }}^{0} & \equiv \frac{12 \pi}{m_{Z^{0}}^{2}} \frac{\Gamma_{e e} \Gamma_{\text {had }}}{\Gamma_{Z^{0}}^{2}}, \\
\Rightarrow \Gamma_{Z^{0}} & =\left(\frac{12 \pi}{m_{Z^{0}}^{2}} \frac{\Gamma_{l \ell} \Gamma_{h a d}}{\sigma_{h a d}^{0}}\right)^{\frac{1}{2}} .
\end{aligned}
$$

\footnotetext{
1 The 'pole' nomenclature denotes a method of establishing the observables of an unstable particle, in this case the $Z^{0}$ boson, using scattering matrix theory. The theory is gauge invariant and model-independent[33].
} 


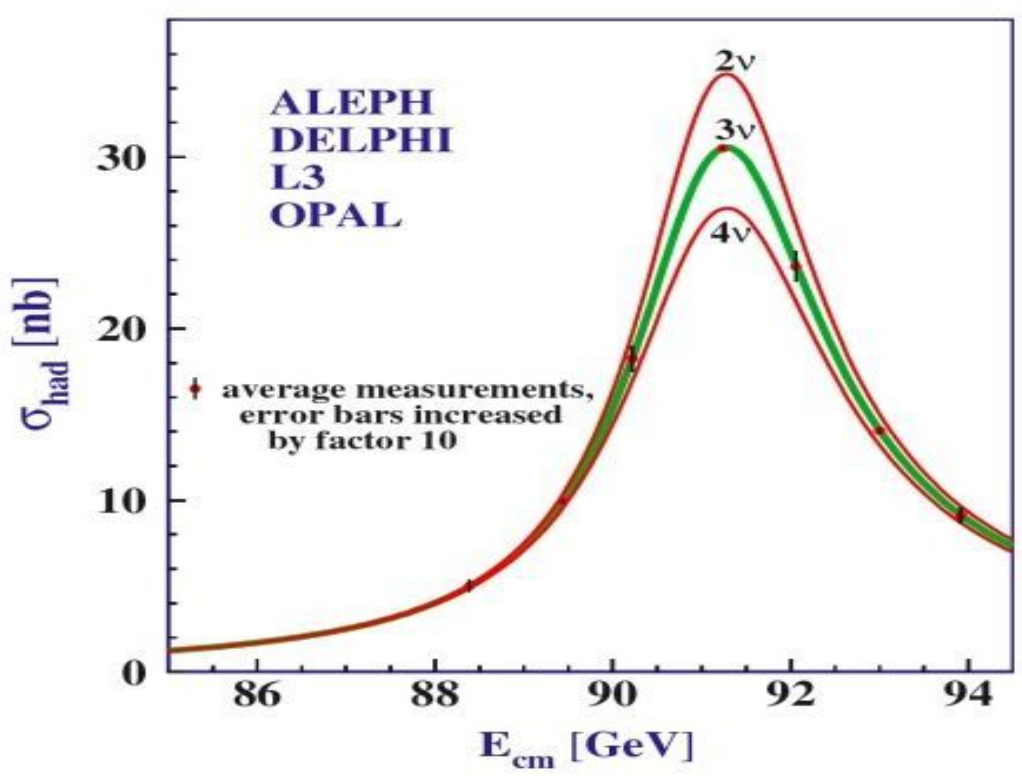

Figure 2.1: Combined measurement from the LEP experiments (ALEPH, DELPHI, L3 and OPAL) that measured the width of the $Z^{0}$ boson [34]. The two red lines show the expected width if the $Z^{0}$ boson coupled to either 2 or 4 neutrinos instead of 3. The result from the LEP experiments for the total number of active light neutrinos is $N_{v}=2.984 \pm 0.008$.

Substituting the expression of $\Gamma_{Z^{0}}$ from eq.2.7 into eq.2.5 gives,

$$
\begin{aligned}
R_{i n v}^{0} & =\frac{\left(\frac{12 \pi}{m_{Z^{0}}^{2}} \frac{\Gamma_{\ell \Gamma_{\text {had }}}}{\sigma_{\text {had }}^{0}}\right)^{\frac{1}{2}}-\Gamma_{\text {had }}}{\Gamma_{\ell l}}-3, \\
& =\left(\frac{12 \pi}{m_{Z^{0}}^{2}} \frac{R_{\ell l}^{0}}{\sigma_{\text {had }}^{0}}\right)^{\frac{1}{2}}-R_{l l}^{0}-3,
\end{aligned}
$$

where $R_{\ell l}^{0}$ represents the hadronic to lepton partial width ratio, defined as $\Gamma_{\text {had }} / \Gamma_{\ell l}$.

The measured value of $R_{i n v}^{0}$, as given by the expression above, is $5.943 \pm$ $0.016[35]$. The importance of the experimentally measurable quantity $R_{i n v}^{0}$ is that if the missing partial width given in eq.2.3) is due to neutrinos, then the expression can be rewritten in terms of the SM derived ratio of the neutrino to lepton partial width

$$
R_{i n v}^{0} \equiv \frac{\Gamma_{i n v}}{\Gamma_{\ell \ell}}=N_{v}\left(\frac{\Gamma_{v \bar{v}}}{\Gamma_{\ell \ell}}\right)_{S M},
$$


where the SM value of $\Gamma_{v \bar{v}} / \Gamma_{\ell}$ is $1.99125 \pm 0.00083$. Substituting the $\Gamma_{\bar{v} \bar{v}} / \Gamma_{\ell \ell}$ value into the previous expression yields the number of neutrinos that couple to the $Z, N_{v}=2.984 \pm 0.008$. The combined LEP data and the 2, 3 and 4 neutrino predictions are shown in Fig. 2.1.

The coupling of only the three known neutrinos to the $Z^{0}$ is an important result in its own right, but also illustrates that a non-interacting neutrino or sterile neutrino would exist outside the framework of the Standard Model. The theory of neutrino oscillation also exists outside the framework of the SM and can act as a vehicle to probe the possible existence of a sterile neutrino.

\subsection{Oscillation Theory}

Experiments probing atmospheric and solar neutrino deficit, such as Super-K[20, 30, 31] and SNO[18], demonstrate a non-zero mass. Oscillation theory has helped accelerator experiments, such as MINOS[36], KEK to Kamioka (K2K)[37], and Cern Neutrinos to Gran Sasso (CNGS)[38] optimize neutrino beams to probe neutrino structure and behavior. In order to make sense of the results of neutrino oscillation experiments and their implications for a sterile neutrino, an understanding of neutrino oscillation is essential.

Neutrinos are detected in their weak or flavor eigenstate, yet propagate in their mass eigenstate. The two bases are related through a unitary matrix akin to the Cabibbo-Kobayashi-Maskawa (CKM) [39, 40] and known as the Pontecorvo-MakiNakagawa-Sakata (PMNS) matrix[14, 16]. In the following section, oscillation probabilities for the three active neutrinos make explicit use of the PMNS matrix, while probabilities with the addition of a theorized sterile neutrino can be found in Sec. 2.2 .3 


\subsubsection{Three neutrino model}

The PMNS mixing matrix for the standard 3 flavor neutrino model can be written in terms that relate the mass to flavor eigenstates. In this approach the unitary mixing matrix, $U$, is written as

$$
\begin{aligned}
\left(\begin{array}{c}
\left|v_{e}\right\rangle \\
\left|v_{\mu}\right\rangle \\
\left|v_{\tau}\right\rangle
\end{array}\right) & =\mathcal{U}\left(\begin{array}{c}
\left|v_{1}\right\rangle \\
\left|v_{2}\right\rangle \\
\left|v_{3}\right\rangle
\end{array}\right) \\
& =\left(\begin{array}{lll}
U_{e 1} & U_{e 2} & U_{e 3} \\
U_{\mu 1} & U_{\mu 2} & U_{\mu 3} \\
U_{\tau 1} & U_{\tau 2} & U_{\tau 3}
\end{array}\right)\left(\begin{array}{c}
\left|v_{1}\right\rangle \\
\left|v_{2}\right\rangle \\
\left|v_{3}\right\rangle
\end{array}\right)
\end{aligned}
$$

where $U_{e 1}$ is the amount of $v_{e}$ flavor in the first mass eigenstate and $U_{\mu 2}$ the amount of $v_{\mu}$ flavor in the second mass eigenstate. For a generic $v_{\alpha} \rightarrow v_{\beta}$ transition, the PMNS matrix elements are used to calculate the oscillation probability, which is conventionally broken up into the $\operatorname{real}(\mathfrak{R})$ and imaginary $(\mathfrak{I})$ portions $s^{2}$ as [41]

$$
\begin{aligned}
P\left(v_{\alpha} \rightarrow v_{\beta}\right)= & \delta_{\alpha \beta}-4 \sum_{i>j} \Re\left(U_{\alpha i}^{*} U_{\beta i} U_{\alpha j} U_{\beta j}^{*}\right) \sin ^{2} \Delta_{i j} \\
& +2 \sum_{i>j} \mathfrak{I}\left(U_{\alpha i}^{*} U_{\beta i} U_{\alpha j} U_{\beta j}^{*}\right) \sin 2 \Delta_{i j} \\
\Delta_{i j} \equiv & \left(m_{i}^{2}-m_{j}^{2}\right) L / 4 E \\
= & \Delta m_{i j}^{2} L / 4 E
\end{aligned}
$$

where $U_{\alpha i}$ is the unitary matrix mixing element of flavor eigenstate $\alpha$ and mass eigenstate $i, L$ is the baseline in $\mathrm{km}, E$ is the energy in $\mathrm{GeV}$ and $m$ is the mass of the neutrino in $\mathrm{eV}$. The mass difference as well as the mixing elements are pictorally presented in Fig. 2.2

\footnotetext{
${ }^{2}$ The full derivation to produce eq. $(2.13)$ is shown in Sec. 2.2 .3 .
} 


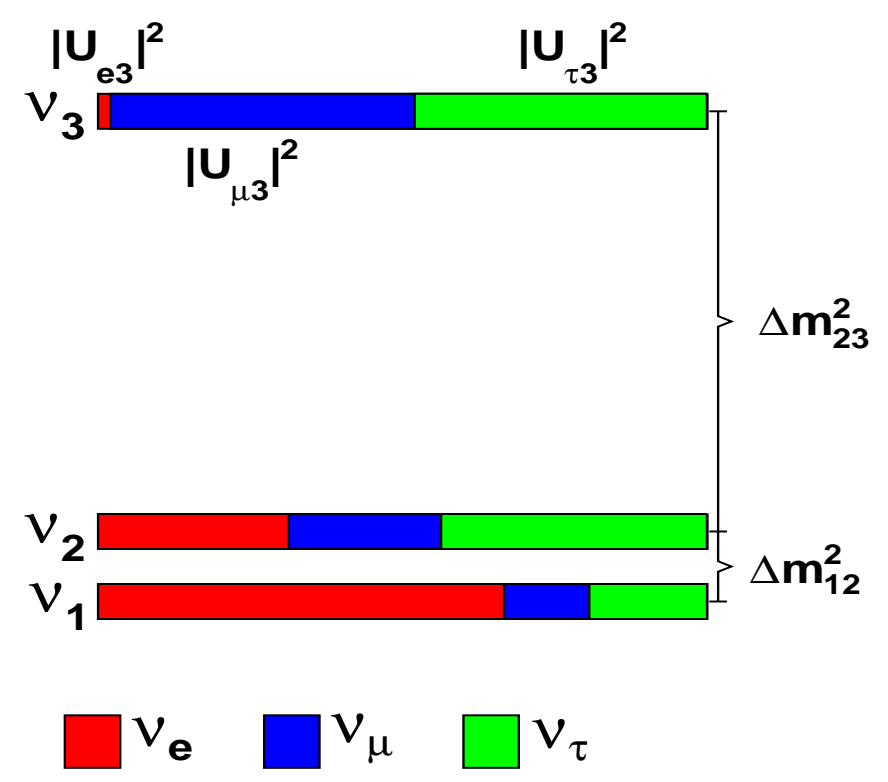

Figure 2.2: 3 flavor neutrino hierarchy, using the normal hierarchy orientation.

In order to formulate MINOS-specific oscillation probabilities, the following substitutions are made:

- $\Delta_{21} \cong 0$ At the baseline and energy of MINOS, the first two mass eigenstates, $m_{1}$ and $m_{2}$, are approximately degenerate.

- $\Delta_{23} \approx \Delta_{31}$ From Fig. 2.2, $\Delta_{31}=\Delta_{21}+\Delta_{32}$.

which, in concert with eq.2.13, produces the transition probability,

$$
P\left(v_{\alpha} \rightarrow v_{\beta}\right) \cong\left|U_{\alpha 3}\right|^{2}\left|U_{\beta 3}\right|^{2} \sin ^{2} \Delta_{31}
$$

While the previous expression calculates the transition probabilities in terms of the matrix elements, they are often written in terms of rotation angles, which 
tranform the expression in eq. 2.12 to

$$
\left(\begin{array}{c}
\left|v_{e}\right\rangle \\
\left|v_{\mu}\right\rangle \\
\left|v_{\tau}\right\rangle
\end{array}\right)=\left[\begin{array}{ccc}
c_{12} c_{13} & s_{12} c_{13} & s_{13} e^{-i \delta} \\
-s_{12} c_{23}-c_{12} s_{23} s_{13} e^{i \delta} & c_{12} c_{23}-s_{12} s_{23} s_{13} e^{i \delta} & s_{23} c_{13} \\
s_{12} s_{23}-c_{12} c_{23} s_{13} e^{i \delta} & c_{12} s_{23}-s_{12} c_{23} s_{13} e^{i \delta} & c_{23} c_{13}
\end{array}\right]\left(\begin{array}{c}
\left|v_{1}\right\rangle \\
\left|v_{2}\right\rangle \\
\left|v_{3}\right\rangle
\end{array}\right)
$$

where $c_{i j}=\cos \theta_{i j}$ and $s_{i j}=\sin \theta_{i j}$ are the weak mixing angles and $\delta$ is the CPviolating phase angle. Substituting the elements of the PMNS matrix in terms of angles into the MINOS specific oscillation probability expression yields,

$$
\begin{aligned}
P\left(v_{\mu} \rightarrow v_{e}\right) & =4\left|U_{\mu 3}\right|^{2}\left|U_{e 3}\right|^{2} \sin ^{2} \Delta_{31} \\
& =4 \sin ^{2} \theta_{23} \cos ^{2} \theta_{13} \sin ^{2} \theta_{13} \sin ^{2} \Delta_{31}, \\
& =4 \sin ^{2} \theta_{23}\left(\frac{\sin 2 \theta_{13}}{2}\right)^{2} \sin ^{2} \Delta_{31}, \\
& =\sin ^{2} \theta_{23} \sin ^{2} 2 \theta_{13} \sin ^{2} \Delta_{31}, \\
P\left(v_{\mu} \rightarrow v_{\tau}\right) & =4\left|U_{\mu 3}\right|^{2}\left|U_{\tau 3}\right|^{2} \sin ^{2} \Delta_{31} \\
& =4 \sin ^{2} \theta_{23} \cos ^{2} \theta_{13} \cos ^{2} \theta_{23} \cos ^{2} \theta_{13} \sin ^{2} \Delta_{31}, \\
& =\sin ^{2} 2 \theta_{23} \cos ^{4} \theta_{13} \sin ^{2} \Delta_{31}, \\
P\left(v_{\mu} \rightarrow v_{\mu}\right) & =1-P\left(v_{\mu} \rightarrow v_{e}\right)-P\left(v_{\mu} \rightarrow v_{\tau}\right) .
\end{aligned}
$$

The expressions derived above are the common transition probabilities for neutrino oscillations in vacuo, where an illustration of the $v_{\mu} \rightarrow v_{\mu}$ probability as a function of energy is shown in the top plot of Fig. 2.3. For MINOS, the three oscillation probabilities are dependent on one mass squared splitting, $\Delta m_{31}^{2}$, and two mixing angles, $\theta_{13}$ and $\theta_{23}$, in addition to the baseline and neutrino energy. When the neutrino path passes through matter, the transition probabilities differ from those in vacuo, and further treatment is necessary to incorporate the possibility of 

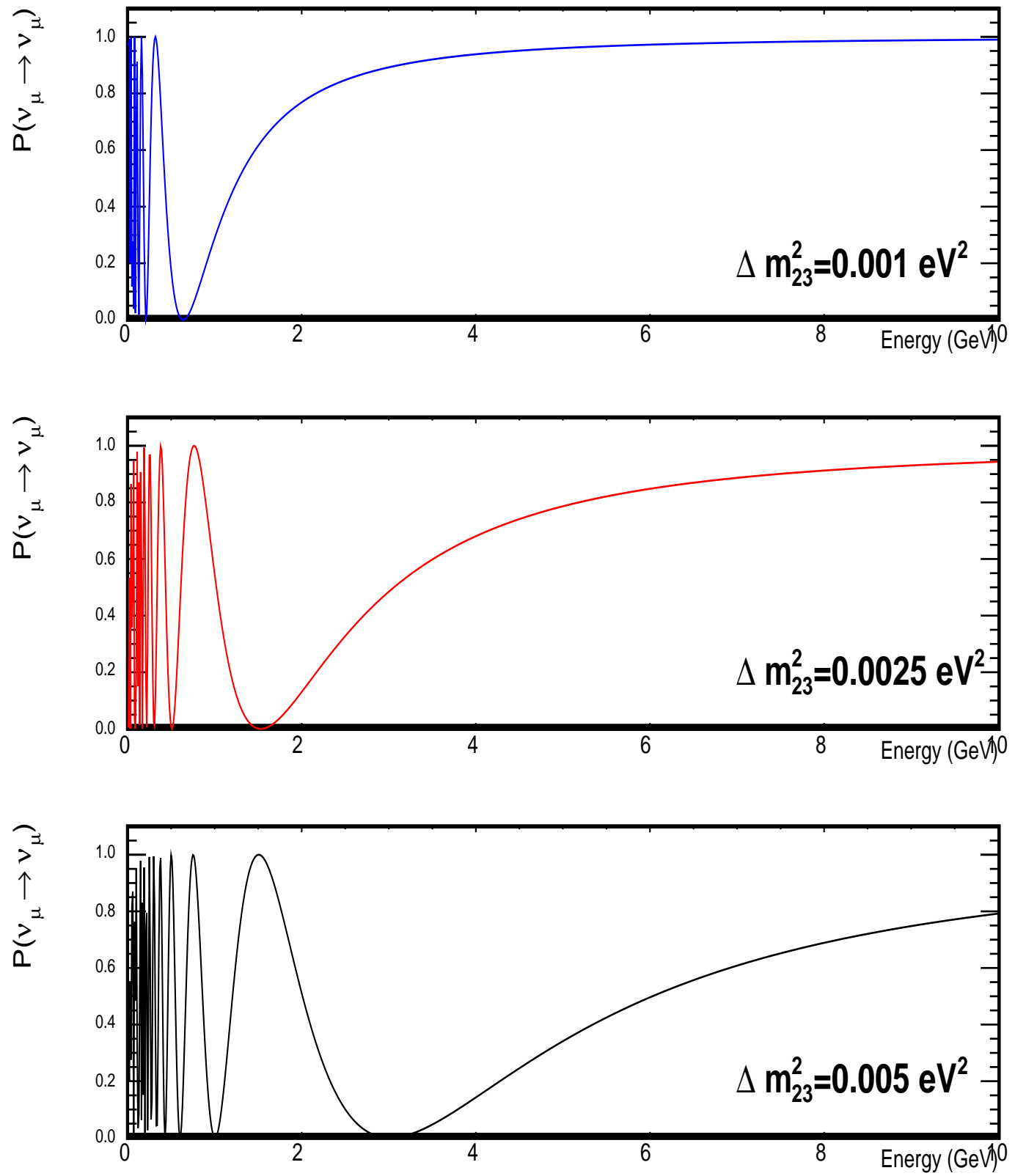

Figure 2.3: Survival probabilities in vacuo of $v_{\mu} \rightarrow v_{\mu}$ for three different values of $\Delta \mathrm{m}_{32}^{2}$ over a $735 \mathrm{~km}$ baseline. The other oscillation parameters are set as $\theta_{31}=0$, $\theta_{23}=\pi / 4, \theta_{21}=0.61$, and $\Delta \mathrm{m}_{21}^{2}=7.59 \times 10^{-5} \mathrm{eV}^{2}$. The value of $\Delta \mathrm{m}_{23}^{2}$ determines the location of the first oscillation dip. 
$v_{e}$ charged current elastic scattering with electrons, described by Wolfenstein[42] and Mikheev \& Smirnov[43][44] (MSW).

\subsubsection{Neutrino Oscillations in Matter - MSW}

Mass difference, energy and distance are not the only factors that contribute to neutrino oscillation. The $v_{e}$ and $\bar{v}_{e}$ charged current elastic forward scattering with electrons in atoms, shown in Fig. 2.4, modifies the neutrino oscillation probabilities that were derived in Sec. 2.2.1. The effect is seen in solar neutrinos and depends on energy, but the probability difference between propagation through matter and in vacuo is small for MINOS due to the low density of the rock in the earth as well as the fact that the neutrino beam is mainly composed of muon, and not electron, neutrinos. This being said, the little amount that the MSW effect does influence MINOS comes in the form of the $v_{e}$ appearance, which is the signal for the measurement of $\theta_{13}$ and more importantly for this thesis, a potentially small but notable background in the search for sterile neutrinos which will be covered in depth in Sec. 5.6.7.

The modified transition probabilities [45, 46] for the 3 Flavor model, when accounting for the MSW effect is

$$
\begin{aligned}
P\left(\mathrm{v}_{\mu} \rightarrow \mathrm{v}_{e}\right)= & s_{23}^{2} \sin ^{2}\left(2 \theta_{13}\right)\left(\frac{2 \Delta_{13}}{B}\right)^{2} \sin ^{2}\left(\frac{B L}{2}\right) \\
& +c_{23}^{2} \sin ^{2}\left(2 \theta_{12}\right)\left(\frac{2 \Delta_{12}}{A}\right)^{2} \sin ^{2}\left(\frac{A L}{2}\right) \\
& +J \frac{2 \Delta_{12}}{A} \frac{2 \Delta_{13}}{B} \sin \left(\frac{A L}{2}\right) \sin \left(\frac{B L}{2}\right) \cos \left(\delta-\Delta_{13} L\right)
\end{aligned}
$$




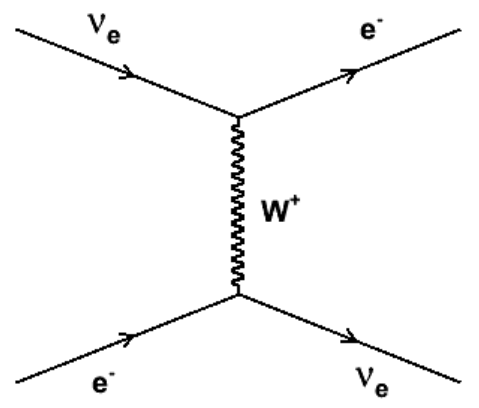

(a)

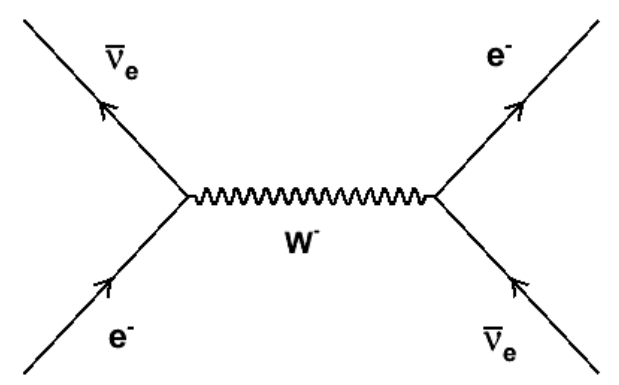

(b)

Figure 2.4: Feynman diagrams of the charged current forward scattering of electrons with (a) electron neutrinos and (b) electron anti-neutrinos.

where $\delta$ is the CP-violating phase factor and

$$
\begin{aligned}
A & =\sqrt{2} G_{f} n_{e} \\
B & =\left|A-2 \Delta_{13}\right| \\
J & =c_{13} \sin \left(2 \theta_{12}\right) \sin \left(2 \theta_{23}\right) \sin \left(2 \theta_{13}\right)
\end{aligned}
$$

where $G_{f}$ is the Fermi coupling and $n_{e}$ is the density of electrons that the neutrinos will encounter. This is a first order perturbative expansion that works for the MINOS energy spectrum, but must be modified in order to constrain all the transition probabilities to be in the physical range $0-1$. For further discussion see appendix A.

Taking into account matter affects for the NuMI beam not only introduces a dependence on the density of electrons, but also the value of the CP-violating phase 

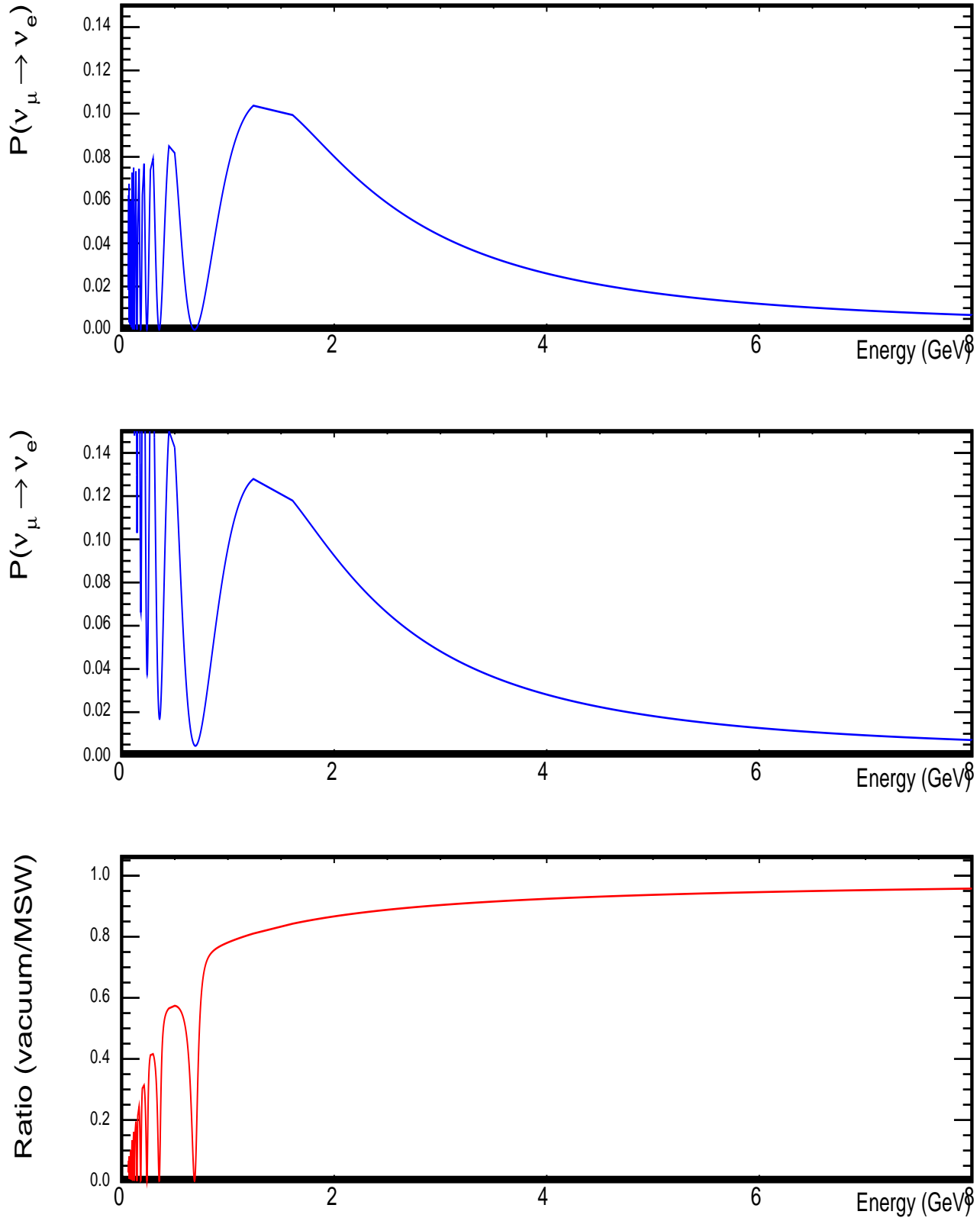

Figure 2.5: Oscillation probabilities for $v_{\mu} \rightarrow v_{e}$. The values used to construct these plots are shown in Table 2.1. The top plot is for vacuum, the middle includes the effects of the NuMI neutrino beam traveling through matter, while the bottom plot shows the ratio of vacuum/matter probability. The oscillation probabilities including matter effects is a perturbative expansion, which introduces non-physical behavior at low energies, $<0.2 \mathrm{GeV}$. Excluding the low energy region, the MSW effect causes a $\sim 10 \%$ increase in the likelihood of $v_{e}$ appearance. 


\begin{tabular}{|c||c|c|}
\hline Parameter & Value & uncertainties \\
\hline$\theta_{13}$ & 0.21 & $<0.21$ at $90 \%$ C.L. \\
$\theta_{12}$ & 0.61 & ${ }_{-0.024}^{+0.028}$ \\
$\theta_{23}$ & $\pi / 4$ & ${ }_{-0.16}$ at $90 \%$ C.L. \\
$\delta_{c p}$ & $3 \pi / 2$ & unbounded \\
$\Delta m_{12}^{2}$ & $7.59 \times 10^{-5} \mathrm{eV}^{2}$ & $\pm 0.21 \times 10^{-5} \mathrm{eV}^{2}$ \\
$\Delta m_{23}^{2}$ & $2.38 \times 10^{-3} \mathrm{eV}^{2}$ & $\pm 0.1 \times 10^{-3} \mathrm{eV}^{2}$ \\
\hline
\end{tabular}

Table 2.1: Values used to make the oscillation plots shown in Fig. 2.5. The value for $\theta_{13}$ coincides with the CHOOZ limit[47], while the $\Delta m_{12}^{2}$ and $\theta_{12}$ values are from the KamLAND and solar best fits[48]. The $\theta_{23}$ is the Super-K best fit value while the $\Delta m_{23}^{2}$ is a measurement made by the MINOS NC Analysis group. Though the value of $\delta_{c p}$ is unconstrained between $0-2 \pi$ the value of $3 \pi / 2$ maximizes the $\mathrm{CP}$-violating contribution.

factor in the third expression of eq.2.26. While $n_{e}$ can be calculated using the average density of the earth coupled with the average number of electrons in matter, the value for $\delta$ is unconstrained between $0-2 \pi$. Ultimately, introducing the MSW effect enhances the accuracy of neutrino oscillation probabilities, shown in Fig. 2.5 even considering the minimal constraint on values for the extra parameters introduced.

\subsubsection{Oscillation including a sterile neutrino}

To formulate neutrino oscillation probabilities including a sterile neutrino, it is best to start from the basic tenets.

Neutrino propagation takes place in terms of the mass eigenstates, and the relationship between the mass and flavor eigenstates is given by

$$
\left|v_{\alpha}\right\rangle=\sum_{j} U_{\alpha j}^{*}\left|v_{j}\right\rangle
$$

where $\alpha$ denotes the flavor eigenstate and $j$ denotes the mass eigenstate. Addition of a time evolution operator gives a further representation of how neutrinos change flavor. 


$$
\begin{aligned}
\left|\mathrm{v}_{j}, t\right\rangle & =e^{-i H t}\left|\mathrm{v}_{j}, 0\right\rangle \\
& =e^{-i\left(E_{j} t-p_{j} L\right)}\left|\mathrm{v}_{j}, 0\right\rangle
\end{aligned}
$$

$E_{j}$ and $p_{j}$ represent the neutrino energy and momentum in the lab reference frame respectively. Assuming that the momentum of the neutrino is much larger than the mass and that all mass eigenstates have common momentum $p$, then the energy term can be rewritten as $E_{j}=\sqrt{p^{2}+m_{j}^{2}} \approx p+m_{j}^{2} / 2 p$ using the binomial expansion, $(1+x)^{y} \approx 1+x y$.

Because the neutrinos of interest are very relativistic, $t \approx L$. Using these expressions, eq. 2.30 can be rewritten as

$$
\left|v_{\alpha}\right\rangle=\sum_{j} U_{\alpha j}^{*} e^{-i\left(m_{j}^{2} / 2 E\right) L}\left|v_{j}, 0\right\rangle
$$

Armed with expressions that detail the relationship between the neutrino flavor and mass eigenstates as well as the evolution of flavor change, it is possible to work through the mathematics to calculate the probability of a neutrino of flavor $\alpha$ to oscillate to flavor $\beta$.

$$
\begin{aligned}
P\left(v_{\alpha} \rightarrow v_{\beta}\right) & =\left|\left\langle v_{\beta} \mid v_{\alpha}\right\rangle\right|^{2}, \\
& =\left|\sum_{j} U_{\alpha j}^{*} U_{\beta j} e^{-i m_{j}^{2} L / 2 E}\right|^{2} .
\end{aligned}
$$

The probability in eq. 2.35) can be expanded in a 3 active plus $\mathrm{N}$ sterile neutrino scenario, which for the following derivation will use a $3+1$ model. In this model the probability can be expanded as 


$$
\begin{aligned}
P\left(v_{\alpha} \rightarrow v_{\beta}\right)=\mid & U_{\alpha 1}^{*} U_{\beta 1} e^{-i m_{1}^{2} L / 2 E}+U_{\alpha 2}^{*} U_{\beta 2} e^{-i m_{2}^{2} L / 2 E} \\
& +U_{\alpha 3}^{*} U_{\beta 3} e^{-i m_{3}^{2} L / 2 E}+\left.U_{\alpha 4}^{*} U_{\beta 4} e^{-i m_{4}^{2} L / 2 E}\right|^{2}
\end{aligned}
$$

Dividing through by $e^{-i m_{1}^{2} L / 2 E}$ casts the probability in terms of mass squared differences, instead of absolute masses. The new phase factors containing the mass squared splittings are rewritten as $\Delta_{j k}=\left(m_{j}^{2}-m_{k}^{2}\right) L / 4 E$

$$
\begin{aligned}
P\left(v_{\alpha} \rightarrow v_{\beta}\right)=\mid & U_{\alpha 1}^{*} U_{\beta 1}+U_{\alpha 2}^{*} U_{\beta 2} e^{-i 2 \Delta_{21}} \\
& +U_{\alpha 3}^{*} U_{\beta 3} e^{-i 2 \Delta_{31}}+\left.U_{\alpha 4}^{*} U_{\beta 4} e^{-i 2 \Delta_{41}}\right|^{2}
\end{aligned}
$$

Using unitarity, in the context of the probability above, $\sum_{j} U_{\alpha j}^{*} U_{\beta j}=1$ so that $U_{\alpha 1}^{*} U_{\beta 1}=1-U_{\alpha 2}^{*} U_{\beta 2}-U_{\alpha 3}^{*} U_{\beta 3}-U_{\alpha 4}^{*} U_{\beta 4}$. Making the $U_{\alpha 1}^{*} U_{\beta 1}$ substitution, the probability becomes

$$
\begin{aligned}
P\left(v_{\alpha} \rightarrow v_{\beta}\right)= & \mid 1+U_{\alpha 2}^{*} U_{\beta 2}\left(e^{-i 2 \Delta_{21}}-1\right)+U_{\alpha 3}^{*} U_{\beta 3}\left(e^{-i 2 \Delta_{31}}-1\right) \\
& +\left.U_{\alpha 4}^{*} U_{\beta 4}\left(e^{-i 2 \Delta_{41}}-1\right)\right|^{2}
\end{aligned}
$$

Rearranging terms and making use of the relation $\sin (x)=\frac{e^{i x}-e^{-i x}}{2 i}$, the probability becomes 


$$
\begin{aligned}
P\left(v_{\alpha} \rightarrow v_{\beta}\right)= & \mid 1+U_{\alpha 2}^{*} U_{\beta 2} e^{-i \Delta_{21}}\left(e^{-i \Delta_{21}}-e^{i \Delta_{21}}\right) \\
& +U_{\alpha 3}^{*} U_{\beta 3} e^{-i \Delta_{31}}\left(e^{-i \Delta_{31}}-e^{i \Delta_{31}}\right) \\
& +\left.U_{\alpha 4}^{*} U_{\beta 4} e^{-i \Delta_{41}}\left(e^{-i \Delta_{41}}-e^{i \Delta_{41}}\right)\right|^{2}, \\
= & \mid 1+U_{\alpha 2}^{*} U_{\beta 2} e^{-i \Delta_{21}}\left(-2 i \sin \Delta_{21}\right)+U_{\alpha 3}^{*} U_{\beta 3} e^{-i \Delta_{31}}\left(-2 i \sin \Delta_{31}\right) \\
& +\left.U_{\alpha 4}^{*} U_{\beta 4} e^{-i \Delta_{41}}\left(-2 i \sin \Delta_{41}\right)\right|^{2} .
\end{aligned}
$$

Pulling out the factor of $e^{-i \Delta_{21}} / 2 i$,

$$
\begin{aligned}
P\left(v_{\alpha} \rightarrow v_{\beta}\right)= & 4 \mid \frac{e^{i \Delta_{21}}}{2 i}-U_{\alpha 2}^{*} U_{\beta 2} \sin \Delta_{21}-U_{\alpha 3}^{*} U_{\beta 3} e^{-i \Delta_{32}} \sin \Delta_{31} \\
& -\left.U_{\alpha 4}^{*} U_{\beta 4} e^{-i \Delta_{42}} \sin \Delta_{41}\right|^{2}
\end{aligned}
$$

This expression can be written as

$$
\delta_{\alpha \beta}-4 \sum_{i>j} \Re\left(U_{\alpha i}^{*} U_{\beta i} U_{\alpha j} U_{\beta j}^{*}\right) \sin ^{2} \Delta_{i j}+2 \sum_{i>j} \mathfrak{I}\left(U_{\alpha i}^{*} U_{\beta i} U_{\alpha j} U_{\beta j}^{*}\right) \sin 2 \Delta_{i j}
$$

which is the same as eq. 2.13 in Sec. 2.2.1

Examining flavor oscillations where $\alpha \neq \beta$, we expand eq.2.41) to get the fol- 
lowing expression,

$$
\begin{aligned}
P\left(v_{\alpha} \rightarrow v_{\beta}\right)= & \Re P\left(v_{\alpha} \rightarrow v_{\beta}\right), \\
= & 4\left\{\left|U_{\alpha 2}\right|^{2}\left|U_{\beta 2}\right|^{2} \sin ^{2} \Delta_{21}+\left|U_{\alpha 3}\right|^{2}\left|U_{\beta 3}\right|^{2} \sin ^{2} \Delta_{31}+\left|U_{\alpha 4}\right|^{2}\left|U_{\beta 4}\right|^{2} \sin ^{2} \Delta_{41}\right. \\
& +\left(\left|U_{\alpha 2}^{*} U_{\beta 2} U_{\alpha 3} U_{\beta 3}^{*}\right|+\left|U_{\alpha 3}^{*} U_{\beta 3} U_{\alpha 2} U_{\beta 2}^{*}\right|\right) \sin \Delta_{21} \sin \Delta_{31} \cos \left(\Delta_{32}+\phi_{32}\right) \\
& +\left(\left|U_{\alpha 2}^{*} U_{\beta 2} U_{\alpha 4} U_{\beta 4}^{*}\right|+\left|U_{\alpha 4}^{*} U_{\beta 4} U_{\alpha 2} U_{\beta 2}^{*}\right|\right) \sin \Delta_{21} \sin \Delta_{41} \cos \left(\Delta_{42}+\phi_{42}\right) \\
& \left.+\left(\left|U_{\alpha 3}^{*} U_{\beta 3} U_{\alpha 4} U_{\beta 4}^{*}\right|+\left|U_{\alpha 4}^{*} U_{\beta 4} U_{\alpha 3} U_{\beta 3}^{*}\right|\right) \sin \Delta_{31} \sin \Delta_{41} \cos \left(\Delta_{43}+\phi_{43}\right)\right\} .
\end{aligned}
$$

The $\cos \left(\Delta_{i j}+\phi_{i j}\right)$ terms are a result of the cross terms from eq.(2.41), illustrated in a single example below

$$
\begin{aligned}
& \frac{1}{4} P\left(v_{\alpha} \rightarrow v_{\beta}\right)=\Re\left\{\ldots+\left(U_{\alpha 2}^{*} U_{\beta 2} \sin \Delta_{21}\right)\left(U_{\alpha 3}^{*} U_{\beta 3} e^{-i \Delta_{32}} \sin \Delta_{31}\right)^{*}+\ldots\right\}, \\
& =\Re\left\{\ldots+\left(U_{\alpha 2}^{*} U_{\beta 2} \sin \Delta_{21}\right)\left(U_{\alpha 3} U_{\beta 3}^{*} e^{i \Delta_{32}} \sin \Delta_{31}\right)+\ldots\right\}, \\
& =\Re\left\{\ldots+U_{\alpha 2}^{*} U_{\beta 2} U_{\alpha 3} U_{\beta 3}^{*} \sin \Delta_{21} \sin \Delta_{31} e^{i \Delta_{32}}+\ldots\right\} \text {, } \\
& =\Re\left\{\ldots+U_{\alpha 2}^{*} U_{\beta 2} U_{\alpha 3} U_{\beta 3}^{*} \sin \Delta_{21} \sin \Delta_{31}\left(\cos \left(\Delta_{32}+\phi_{32}\right)+i \sin \left(\Delta_{32}+\phi_{32}\right)\right)+\ldots\right\} \text {, } \\
& =\left\{\ldots+\left|U_{\alpha 2}^{*} U_{\beta 2} U_{\alpha 3} U_{\beta 3}^{*}\right| \sin \Delta_{21} \sin \Delta_{31} \cos \left(\Delta_{32}+\phi_{32}\right)+\ldots\right\} .
\end{aligned}
$$

At the baseline and energy of MINOS, the first two mass eigenstates, $m_{1}$ and $m_{2}$, can be approximated as degenerate, $\Delta_{12} \cong 0$. The approximation is done to get rid of free parameters to which MINOS has no sensitivity. Also, if the mass squared splitting $\Delta_{43}$ is much larger than the atmospheric splitting, then the $\sin ^{2} \Delta_{4 j}$ $(j=1,2,3)$ terms average to $\frac{1}{2}$. This is due to the MINOS baseline being optimized for atmospheric oscillations. A mass splitting significantly larger than the atmo- 
spheric translates to rapid oscillations that would generate a significantly different oscillation probability for neutrinos differing in travelled distance by only meters. This rapid oscillation averages out to $\frac{1}{2}$.

Setting $\Delta_{21}=0$ and $\sin ^{2} \Delta_{41}=\frac{1}{2}$ provides the probability for an $\alpha$ to $\beta$ neutrino flavor transition in a 3+1 framework. This model has been developed by Stephen Parke via conversations about introducing a $v_{\text {sterile }}$ into the mixing matrix, and will be referred to as the Parke model. The probability for this model extended from eq.(2.44) is defined as

$$
\begin{aligned}
P\left(v_{\alpha} \rightarrow v_{\beta}\right)= & 4\left\{\left|U_{\alpha 3}\right|^{2}\left|U_{\beta 3}\right|^{2} \sin ^{2} \Delta_{31}+\frac{1}{2}\left|U_{\alpha 4}\right|^{2}\left|U_{\beta 4}\right|^{2}\right. \\
& \left.+\left(\left|U_{\alpha 3}^{*} U_{\beta 3} U_{\alpha 4} U_{\beta 4}^{*}\right|+\left|U_{\alpha 4}^{*} U_{\beta 4} U_{\alpha 3} U_{\beta 3}^{*}\right|\right) \sin \Delta_{31} \sin \Delta_{41} \cos \left(\Delta_{43}+\phi_{43}\right)\right\} .
\end{aligned}
$$

The parameters at this stage of the Parke model are the elements of the mixing matrix for the various flavor and mass eigenstates, one phase $\phi_{43}$, and three mass squared splittings $\Delta_{41}, \Delta_{43}$ and $\Delta_{31}$.

Use of trigonometric expressions reduces the three mass squared splittings in the expression to only $\Delta_{31}$. Examining only the sin and cos factors of the interference term, where $\phi=\phi_{43}$, yields

$$
\begin{aligned}
\sin \Delta_{31} \sin \Delta_{41} \cos \left(\Delta_{43}+\phi\right)= & \sin \Delta_{31} \sin \Delta_{41}\left\{\cos \Delta_{43} \cos \phi-\sin \Delta_{43} \sin \phi\right\} \\
= & \sin \Delta_{31} \sin \Delta_{41}\left\{\cos \Delta_{41}-\Delta_{31}\right) \cos \phi \\
& \left.-\sin \left(\Delta_{41}-\Delta_{31}\right) \sin \phi\right\} \\
= & \sin \Delta_{31} \sin \Delta_{41}\left\{\left(\cos \Delta_{41} \cos \Delta_{31}+\sin \Delta_{41} \sin \Delta_{31}\right) \cos \phi\right. \\
& \left.-\left(\sin \Delta_{41} \cos \Delta_{31}-\sin \Delta_{31} \cos \Delta_{41}\right) \sin \phi\right\} .
\end{aligned}
$$

The probability expression no longer contains any terms depending on $\Delta_{43}$. 
Keeping in mind that this is a phenomenological model based on each $\Delta_{4 j}$ term being greater than the atmospheric splitting, we know that $\sin 2 \Delta_{41}$ oscillates very rapidly and averages out to zero. Expanding the expression and grouping the $\sin \phi$ and $\cos \phi$ terms gives

$\sin \Delta_{31} \sin \Delta_{41} \cos \left(\Delta_{43}+\phi\right)=\left(\sin \Delta_{31} \sin \Delta_{41} \cos \Delta_{31} \cos \Delta_{41}+\sin ^{2} \Delta_{31} \sin ^{2} \Delta_{41}\right)^{\frac{1}{2}} \cos \phi$

$$
\begin{aligned}
& -\left(\sin ^{2} \Delta_{41} \sin _{31}^{\frac{1}{2}} \cos \Delta_{31}-\sin ^{2} \Delta_{31} \sin \Delta_{41} \cos \Delta_{41}\right) \sin \phi \\
= & \frac{\sin 2 \Delta_{31}}{2} \frac{\sin 2 \Delta_{41}}{2} \cos \phi+\frac{\sin ^{2} \Delta_{31}}{2} \cos \phi \\
& -\frac{\sin 2 \Delta_{31}}{2} \sin \phi+\sin ^{2} \Delta_{31} \frac{\sin 2 \Delta_{41}}{2} \sin \phi \\
= & \frac{\sin ^{2} \Delta_{31}}{2} \cos \phi-\frac{\sin 2 \Delta_{31}}{4} \sin \phi .
\end{aligned}
$$

The $\alpha \neq \beta$ probability, building from eq. 2.50, is now in terms of only $\Delta_{31}, \phi$ and the elements of the mixing matrix.

$$
\begin{aligned}
P\left(v_{\alpha} \rightarrow v_{\beta}\right)= & 4\left\{\left|U_{\alpha 3}\right|^{2}\left|U_{\beta 3}\right|^{2} \sin ^{2} \Delta_{31}+\frac{1}{2}\left|U_{\alpha 4}\right|^{2}\left|U_{\beta 4}\right|^{2}\right. \\
& +\overbrace{\left(\left|U_{\alpha 3}^{*} U_{\beta 3} U_{\alpha 4} U_{\beta 4}^{*}\right|+\left|U_{\alpha 4}^{*} U_{\beta 4} U_{\alpha 3} U_{\beta 3}^{*}\right|\right)}^{2\left|U_{\alpha 3}\right|\left|U_{\beta 3}\right|\left|U_{\alpha 4}\right|\left|U_{\beta 4}\right|}\left(\frac{\sin ^{2} \Delta_{31}}{2} \cos \phi-\frac{\sin 2 \Delta_{31}}{4} \sin \phi\right)\}, \\
= & 4\left|U_{\alpha 3}\right|^{2}\left|U_{\beta 3}\right|^{2} \sin ^{2} \Delta_{31}+2\left|U_{\alpha 4}\right|^{2}\left|U_{\beta 4}\right|^{2} \\
& +4\left|U_{\alpha 3}\right|\left|U_{\beta 3}\right|\left|U_{\alpha 4}\right|\left|U_{\beta 4}\right|\left(\sin ^{2} \Delta_{31} \cos \phi-\frac{\sin 2 \Delta_{31}}{2} \sin \phi\right) .
\end{aligned}
$$

When examining transition probabilities, such as $v_{\mu} \rightarrow v_{e}$, a sense of magnitude needs to be taken into account to minimize extraneous parameters. Various terms are classified as 'small' and 'not small', and only the terms of leading order (quadratic 'small') are kept, while the others are dropped from the expression. 
The 'small' terms of note in the model are $U_{e 3}, U_{e 4}, U_{\mu 4}$ and $U_{\tau 4}$. The amount of sterile in the $4^{\text {th }}$ mass eigenstate is approximately one, i.e. $\left|U_{s 4}\right| \approx 1$. It is now possible to directly calculate the probabilities $P\left(\mathrm{v}_{\mu} \rightarrow \mathrm{v}_{e}\right)$ and $P\left(\mathrm{v}_{\mu} \rightarrow \mathrm{v}_{s}\right)$ using eq.2.57), while using eq. 2.13 to calculate $P\left(v_{\mu} \rightarrow v_{\mu}\right)$ and then use unitarity to obtain $P\left(v_{\mu} \rightarrow v_{\tau}\right)$. The factor

$$
\Phi=\left(\sin ^{2} \Delta_{31} \cos \phi-\frac{\sin 2 \Delta_{31}}{2} \sin \phi\right)
$$

in the following equations represents the $\mathrm{CP}$ violating phase terms.

$$
\begin{aligned}
P\left(v_{\mu} \rightarrow v_{e}\right)= & 4\left|U_{\mu 3}\right|^{2}\left|U_{e 3}\right|^{2} \sin ^{2} \Delta_{31}+2\left|U_{\mu 4}\right|^{2}\left|U_{e 4}\right|^{2^{2}} \\
& +4\left|U_{\mu 3}\right|\left|U_{e 3}\right|\left|U_{\mu 4}+\right| U_{e 4} \mid \Phi \\
= & 4\left|U_{\mu 3}\right|^{2}\left|U_{e 3}\right|^{2} \sin ^{2} \Delta_{31} \\
P\left(v_{\mu} \rightarrow v_{s}\right)= & 4\left|U_{\mu 3}\right|^{2}\left|U_{s 3}\right|^{2} \sin ^{2} \Delta_{31}+2\left|U_{\mu 4}\right|^{2}\left|U_{s 4}\right|^{2^{-1}} \\
& +4\left|U_{\mu 3}\right|\left|U_{s 3}\right|\left|U_{\mu 4}\right|\left|U_{s 4}\right| \stackrel{1}{1} \\
= & 4\left|U_{\mu 3}\right|^{2}\left|U_{s 3}\right|^{2} \sin ^{2} \Delta_{31}+2\left|U_{\mu 4}\right|^{2}+4\left|U_{\mu 3}\right|\left|U_{s 3}\right|\left|U_{\mu 4}\right| \Phi .(2.62)
\end{aligned}
$$


Using eq.2.13, the $v_{\mu}$ to $v_{\mu}$ probability is

$$
\begin{aligned}
P\left(v_{\mu} \rightarrow v_{\mu}\right)= & 1-4 \sum_{i>j}\left|U_{\mu i}\right|^{2}\left|U_{\mu j}\right|^{2} \sin ^{2} \Delta_{i j} \\
= & 1-4\left\{\left|U_{\mu 2}\right|^{2}\left|U_{\mu 1}\right|^{2} \sin ^{2} \Delta_{21}\right. \\
& +\left|U_{\mu 3}\right|^{2}\left|U_{\mu 1}\right|^{2} \sin ^{2} \Delta_{31} \\
& +\left|U_{\mu 4}\right|^{2}\left|U_{\mu 1}\right|^{2} \sin ^{2} \Delta_{41} \\
& +\left|U_{\mu 3}\right|^{2}\left|U_{\mu 2}\right|^{2} \sin ^{2} \Delta_{32} \\
& +\left|U_{\mu 4}\right|^{2}\left|U_{\mu 2}\right|^{2} \sin ^{2} \Delta_{42}{ }^{\frac{1}{2}} \\
& \left.+\left|U_{\mu 4}\right|^{2}\left|U_{\mu 3}\right|^{2} \sin ^{2} \Delta_{43}{ }^{\frac{1}{2}}\right\} \\
= & 1-4\left|U_{\mu 3}\right|^{2}\left|U_{\mu 1}\right|^{2} \sin ^{2} \Delta_{31}-2\left|U_{\mu 4}\right|^{2}\left|U_{\mu 1}\right|^{2} \\
& -4\left|U_{\mu 3}\right|^{2}\left|U_{\mu 2}\right|^{2} \sin ^{2} \Delta_{32}-2\left|U_{\mu 4}\right|^{2}\left|U_{\mu 2}\right|^{2}-2\left|U_{\mu 4}\right|^{2}\left|U_{\mu 3}\right|^{2} .
\end{aligned}
$$

For a long baseline neutrino experiment there is a degeneracy between $\Delta_{31}$ and $\Delta_{32}$, such that $\Delta_{31} \approx \Delta_{32}$. Collecting the terms and making the $\Delta_{31}$ substitution the probability becomes

$$
\begin{aligned}
P\left(v_{\mu} \rightarrow v_{\mu}\right)= & 1-4\left|U_{\mu 3}\right|^{2}\left(\left|U_{\mu 1}\right|^{2}+\left|U_{\mu 2}\right|^{2}\right) \sin ^{2} \Delta_{31} \\
& -2\left|U_{\mu 4}\right|^{2}\left(\left|U_{\mu 1}\right|^{2}+\left|U_{\mu 2}\right|^{2}+\left|U_{\mu 3}\right|^{2}\right) .
\end{aligned}
$$


Using unitarity, and dropping all non-leading order terms, the expression becomes:

$$
\begin{aligned}
P\left(v_{\mu} \rightarrow v_{\mu}\right)= & -4\left|U_{\mu 3}\right|^{2}\left(\left|U_{\mu 1}\right|^{2}+\left|U_{\mu 2}\right|^{2}\right) \sin ^{2} \Delta_{31} \\
& -2\left|U_{\mu 4}\right|^{2}\left(1-\left|U_{\mu 4}\right|^{2}\right) \\
= & 1-4\left|U_{\mu 3}\right|^{2}\left(\left|U_{\mu 1}\right|^{2}+\left|U_{\mu 2}\right|^{2}\right) \sin ^{2} \Delta_{31} \\
& -2\left|U_{\mu 4}\right|^{2}-2\left|U_{\mu 4}\right|^{2}\left|U_{\mu 4}\right|^{2} 0 \\
= & 1-4\left|U_{\mu 3}\right|^{2}\left(\left|U_{\mu 1}\right|^{2}+\left|U_{\mu 2}\right|^{2}\right) \sin ^{2} \Delta_{31}-2\left|U_{\mu 4}\right|^{2} \\
= & 1-4\left|U_{\mu 3}\right|^{2}(\overbrace{\left.\left|U_{\mu 1}\right|^{2}+\left|U_{\mu 2}\right|^{2}+\left|U_{\mu 3}\right|^{2}+\left|U_{\mu 4}\right|^{2}-\left|U_{\mu 3}\right|^{2}-\left|U_{\mu 4}\right|^{2}\right) \sin ^{2} \Delta_{31}}^{1} \\
& -2\left|U_{\mu 4}\right|^{2} \\
= & 1-4\left|U_{\mu 3}\right|^{2}\left(1-\left|U_{\mu 3}\right|^{2}\right) \sin ^{2} \Delta_{31}+4\left|U_{\mu 4}\right|^{2}\left|U_{\mu 3}\right|^{2} \sin ^{2} \Delta_{31}-2\left|U_{\mu 4}\right|^{2} \\
= & 1-4\left|U_{\mu 3}\right|^{2}\left(1-\left|U_{\mu 3}\right|^{2}\right) \sin ^{2} \Delta_{31}+4\left|U_{\mu 4}\right|^{2}\left(\left|U_{\mu 3}\right|^{2} \sin ^{2} \Delta_{31}-\frac{1}{2}\right)
\end{aligned}
$$


The final transition probabilities of the Parke model can then be written as

$$
\begin{aligned}
P\left(v_{\mu} \rightarrow v_{e}\right)= & 4\left|U_{\mu 3}\right|^{2}\left|U_{e 3}\right|^{2} \sin ^{2} \Delta_{31} \\
P\left(v_{\mu} \rightarrow v_{s}\right)= & 4\left|U_{\mu 3}\right|^{2}\left|U_{s 3}\right|^{2} \sin ^{2} \Delta_{31}+2\left|U_{\mu 4}\right|^{2}+4\left|U_{\mu 3}\right|\left|U_{s 3}\right|\left|U_{\mu 4}\right| \Phi \\
= & 4\left|U_{\mu 3}\right|^{2}\left|U_{s 3}\right|^{2} \sin ^{2} \Delta_{31}+2\left|U_{\mu 4}\right|^{2} \\
& +4\left|U_{\mu 3}\right|\left|U_{s 3}\right|\left|U_{\mu 4}\right|\left(\sin ^{2} \Delta_{31} \cos \phi_{43}-\frac{\sin 2 \Delta_{31}}{2} \sin \phi_{43}\right) \\
P\left(\mathrm{v}_{\mu} \rightarrow v_{\mu}\right)= & 1-4\left|U_{\mu 3}\right|^{2}\left(1-\left|U_{\mu 3}\right|^{2}\right) \sin ^{2} \Delta_{31} \\
& +4\left|U_{\mu 4}\right|^{2}\left(\left|U_{\mu 3}\right|^{2} \sin ^{2} \Delta_{31}-\frac{1}{2}\right) \\
P\left(v_{\mu} \rightarrow v_{\tau}\right)= & 1-P\left(v_{\mu} \rightarrow v_{e}\right)-P\left(v_{\mu} \rightarrow v_{s}\right)-P\left(v_{\mu} \rightarrow v_{\mu}\right)
\end{aligned}
$$

The probabilities for neutrino oscillations in the $3+1$ Parke model have 6 terms: $\left|U_{\mu 3}\right|^{2},\left|U_{e 3}\right|^{2},\left|U_{s 3}\right|, U_{\mu 4}, \phi_{43}$ and $\Delta_{31}$. The Parke model exists in a unique and finely tuned parameter space and should not be taken as the only or even general model to look for sterile neutrinos, but one that is optimized for the MINOS experiment. Other models may be more accurate or complete, but would produce too many free parameters to give MINOS any sensitivity to those that illuminate the nature of sterile neutrinos. This thesis will consider both the standard 3 neutrino model as well as the $3+1$ Parke model. 


\section{Chapter 3}

\section{Neutrino Beam}

The sensitivity of MINOS to neutrino oscillation is highly dependent on the flux and energy of the beam. The beam of muon neutrinos supplied to MINOS comes from the Neutrinos at the Main Injector (NuMI) complex at Fermilab. The high purity muon neutrino beam is the most intense neutrino source on Earth and can produce a variable energy spectrum. The variability allows data taking in different configurations for background studies as well as beam tuning to the most sensitive region for precision measurements of neutrino oscillation parameters.

Rudimentary understanding of the neutrino beam can be broken down into two topics: protons and the NuMI beamline.

\subsection{Protons}

NuMI supplies protons to MINOS in one of two operation modes: 'mixed' and 'NuMI only'. During Tevatron operation, batches of protons are divided between MINOS and the anti-proton source, constituting the 'mixed' mode whereas, during Tevatron downtime, MINOS receives the full share, known as 'NuMI-only' mode. The protons are the result of four separate steps:

I. Create and accelerate $\mathrm{H}^{-}$ions to $0.75 \mathrm{MeV}$ using a Cockroft-Walton ma- 
chine.

II. Accelerate $\mathrm{H}^{-}$ions to $400 \mathrm{MeV}$ using a Linear Accelerator.

III. Strip the electrons from $\mathrm{H}^{-}$to create a proton beam and use the FNAL Booster synchrotron to accelerate the proton beam to $8 \mathrm{GeV}$.

IV. Use a larger and higher energy synchrotron, the Main Injector, to accelerate the proton beam to $120 \mathrm{GeV}$.

The whole process initially produced $2 \times 10^{13}$ protons/pulse for MINOS every 2.4 seconds in mixed-mode. Advances in accelerator physics have increased the intensity to $2.6 \times 10^{13}\left(3.5 \times 10^{13}\right)$ protons for mixed-mode (NuMI only) and reduced the cycle time to $2.2 \mathrm{~s}$ for both modes. The limitations for further increases to protons are related to proton beam losses, creating radiation hazards from the Booster and radiation/machine issues in the Main Injector. The following sections provide a coherent picture of protons from their inception to extraction down the NuMI beamline, detailing where intensity limitations lie as well as steps to overcome the specific challenges.

\subsection{1 $\mathbf{H}^{-}$source/Cockroft-Walton}

The NuMI protons begin as hydrogen gas molecules, and become $\mathrm{H}^{-}$ions through a magnetron plasma source[49, 50]. The initial step is a release of hydrogen from a gas canister into a high electric field produced by an oval cathode, see Fig. 3.1 The field separates the proton and electron of the hydrogen, leaving the proton to congregate on the metal surface of the cathode and the electron to form a dense plasma. The plasma is confined in the gap between the cathode and anode by a magnetic field oriented perpendicular to the electric field. A monolayer of cesium, introduced as a vapor, present on the cathode surface reduces the work function of 
the metal allowing ejected protons to capture two electrons instead of one to make a $\mathrm{H}^{-}$ion. The ejected protons make $\mathrm{H}^{-}$ions in one of two ways:

- Collision - A proton attached to the cathode is struck by an incoming proton or a thermally excited atom. The initial cathode attached proton is ejected and captures two electrons on its escape path.

- Sputtering - A particle directly strikes the cathode and undergoes multiple collisions within the cesium monolayer or cathode surface. The subsequent collisions eject protons from the metal substrate of the cathode creating a $\mathrm{H}^{-}$ cascade.

Although the $\mathrm{H}^{-}$creation process occurs across the whole cathode, only ions near the ejection aperture continue on to the initial acceleration stage; the CockroftWalton.

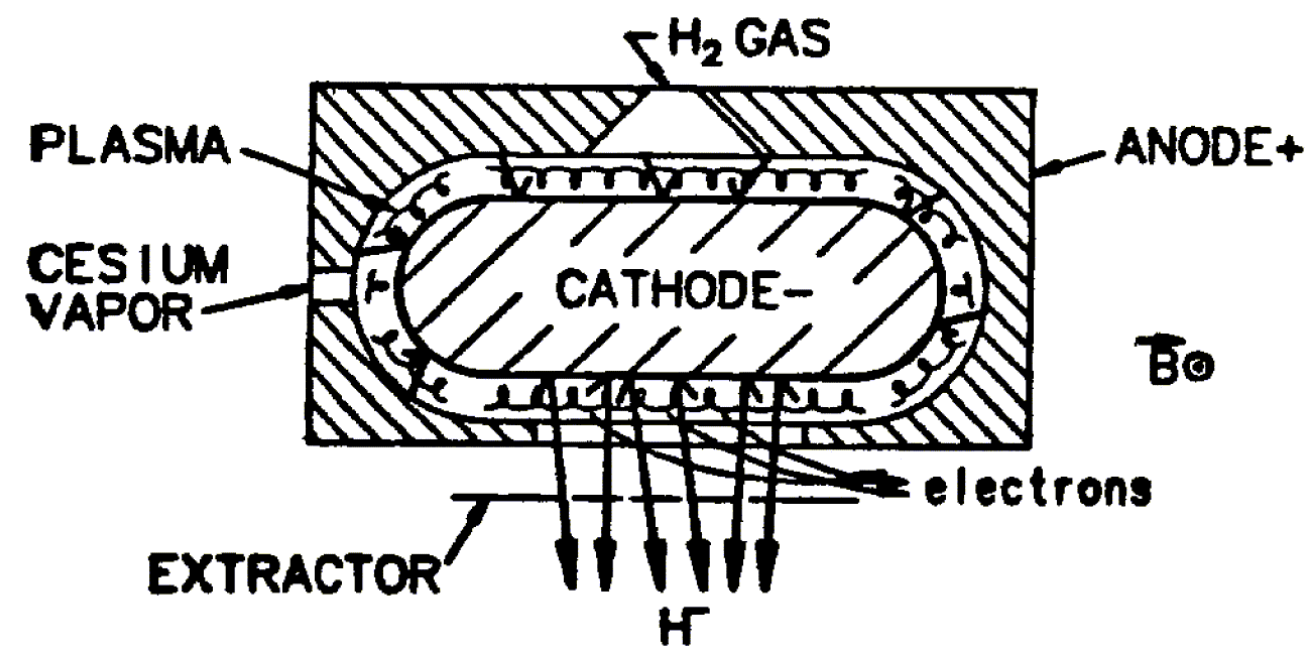

Figure 3.1: The magnetron plasma source converts hydrogen gas into $\mathrm{H}^{-}$ions. The hydrogen is introduced from the top of the diagram, where an electric field separates the proton and electron. The proton is attracted to the cathode while the electrons form a plasma in the gap between cathode and anode. A monolayer of Cesium on the cathode surface reduces the work function of the cathode metal and compels an ejected proton to attract two electrons. $\mathrm{H}^{-}$ions created near the ejection aperture are fed into the Cockroft-Walton accelerator. 
After production in the magnetron, the $\mathrm{H}^{-}$ions are accelerated in the presence of a electrostatic field supplied by a Cockroft-Walton accelerator; a direct current (DC) accelerator using multiple voltage doubling circuits in an escalating ladder. The higher the voltage the stronger the magnetic field that accelerates the $\mathrm{H}^{-}$beam. The Fermilab Cockroft-Walton achieves $750 \mathrm{kV}$ and accelerates the ion beam to $750 \mathrm{keV}$. The ions are funneled into the accelerator from the magnetron plasma source which is housed in a dome attached to the top of the Cockroft-Walton. The ions travel through evacuated glass tubes and exit the tubes into the Linear Accelerator through a series of bending magnets in a transfer line.

\subsubsection{Linac}

The Fermilab Linear Accelerator (Linac) uses staggered sections of electric field for acceleration of the $\mathrm{H}^{-}$beam to $400 \mathrm{MeV}$. Radio frequency (RF) resonators create an oscillating field which produces either a propelling and retarding force on the ions. To provide constant acceleration, there are nine drift chambers in the Linac which shield the beam when the electric field produces an impeding force, and in the gaps between the chambers the beam is advanced. The drift chambers increase in both length and inter-spaced distance to accommodate the increasing energy and wavelength of the ion beam.

The Booster lies 15 feet below the Linac, where a group of 3 dipole magnets bend the beam into the injection septum. At the injection point into the Booster there is a correcting septum magnet which removes the vertical bend, as well as additional magnets which direct the beam parallel to the Booster orbit. The proton beam, already circulating in the Booster, passes through the field free region of the septum magnet, thus avoiding any deflection. The conversion of $\mathrm{H}^{-}$ions to protons, as well as conjoining of circulating proton beam and injected $\mathrm{H}^{-}$beam, is handled by a set of Orbit bump (Orbump) magnets. 

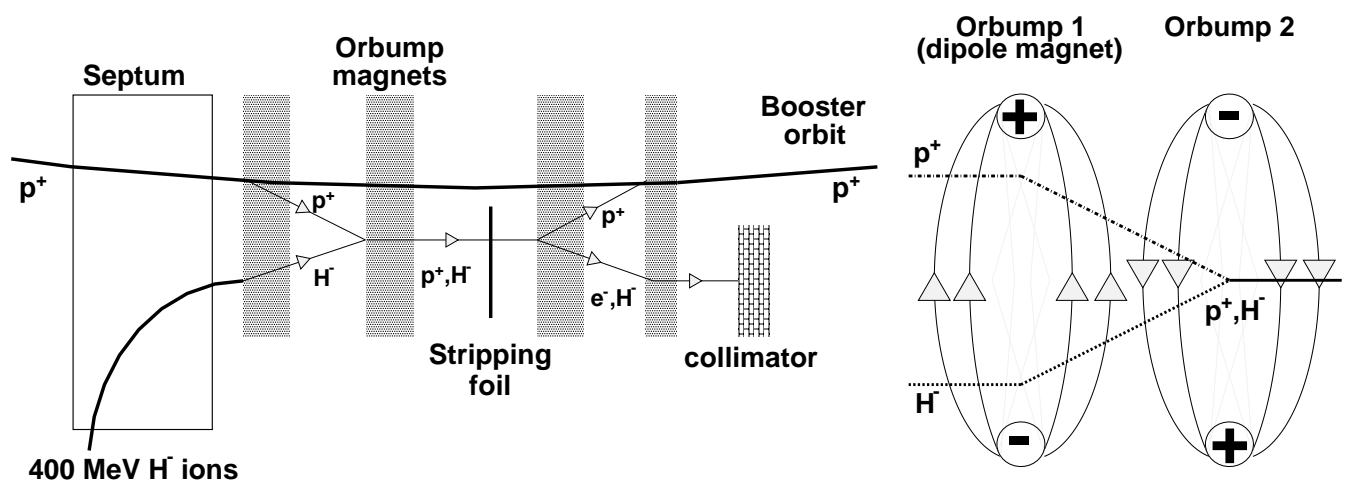

Figure 3.2: The diagram on the left details the injection of the $400 \mathrm{MeV} \mathrm{H}^{-}$ions from the Linac into the Booster and the subsequent conversion to protons by use of a carbon stripping foil. The magnets in the septum direct the $\mathrm{H}^{-}$beam parallel to the Booster orbit. When the Booster is fully loaded the Orbump magnets remain off, keeping the beam in the closed orbit. The diagram on the right is a cartoon of the magnetic field of the dipole Orbump magnets that focus the orbiting $\mathrm{p}^{+}$and injected $\mathrm{H}^{-}$into one beam.

Because of the opposite polarity of the injected beam $\left(\mathrm{H}^{-}\right)$and circulating beam (protons), the same Orbump magnets are used to focus and separate the two (Fig. 3.2(a)). The first set of focusing Orbump magnets combine the two beams into an amalgamated proton/ $\mathrm{H}^{-}$beam (Fig. 3.2(b)). The amalgamation passes through a carbon stripping foil, which removes the surplus electrons. Further Orbump magnets direct the protons into the closed orbit of the Booster while the remaining $\mathrm{H}^{-}$ ions are bent away from the Booster towards a collimator. When the Linac is not injecting beam into the Booster, the Orbump magnets are idle and the beam remains in closed orbit without passing through the stripping foil, minimizing beam loss and scattering.

\subsubsection{Booster}

The injection of beam from the Linac is continuous and fills the circular Booster over multiple revolutions or 'turns'. Each extraction of all the protons in the Booster represents a proton batch, with MINOS batches receiving 8 turns and the 
anti-proton source for the Tevatron receiving 10. Because the number of batches that can be loaded into the downstream Main Injector is fixed (details following in Sec. 3.1.4, the intensity of protons is dependent on how many turns can be injected into the Booster.

Upon injection, protons from the Linac do not initially match the bunch structure required for acceleration in the Booster. The continuous slug of protons from the Linac is adiabatically adjusted into bunches that match the harmonic number of the Booster: 84. When the Booster has been filled, RF cavities capture the beam in the Booster and accelerate it to $8 \mathrm{GeV}$.

The intensity of the protons, or number of turns, is governed by radiation losses in the proton transfer between the Booster and the Main Injector. In the event that Booster buckets 81-84 contain protons, the extraction magnets spray these protons across both the magnets and beam pipe. To reduce radiation, a notch is set, where 2-3 buckets which are synchronized with the rise time of the extraction magnets to the Main Injector are relieved of their protons early in the acceleration cycle. The notch is created by a series of magnets, which knock the protons in the desired buckets into the Booster collimator. Setting a notch allows the rise time of the extraction magnets to coincide with the proton free region of the Booster batch, mitigating radiation in areas frequently requiring human access and maintenance.

Multiple Booster batches are loaded into one cycle of the next part of the accelerator complex: the Main Injector (MI) synchrotron. To avoid beam on beam injection from the Booster to the MI, the beam in the Booster undergoes "cogging". This is a process where the notch is adjusted to coincide with a full extraction of protons in the Booster into an empty region of the Main Injector. To time the notch with the proper extraction point, the path length is altered during acceleration in the Booster. An unfortunate feature of cogging is that the increase in path length increases proton scraping along the beam pipe, which decreases the amount of 
protons. Ideally, the notch would be established in sync with the extraction point early in the acceleration. This removes the need for path length corrections, but the unpredictability of the Booster prevents a perfect prediction between notch and extraction point.

\subsubsection{Main Injector}

The Main Injector (MI) is responsible for accelerating multiple batches from the Booster to $120 \mathrm{GeV}$ for extraction down the NuMI beamline. Because the injected beam and the circulating beam are both protons, the loading structure is different than the Linac to Booster transition. The MI is 7 times the circumference of the Booster in order to provide space for 6 separate batches from the Booster and one notch in a MI cycle. Until January 2008, these regular batches constituted the majority of the operating mode for early MINOS data collecting. In 2008 an intensity breakthrough was achieved through the process of 'slip-stacking'. During slip-stacking 5 batches are injected into the MI with a small voltage offset that makes them decelerate, or 'slip', back through the MI cycle, see Fig. 3.3. Six regular batches with a voltage offset of the opposite sign are then injected so that the buckets in these Booster batches accelerate in the MI cycle. The voltage offset at injection translates to a difference in energy of $\sim 10-20 \mathrm{MeV}$ between protons in the slip-stacked and regular batch. This method of slip-stacking increases the number of Booster batches in one cycle of the MI to 5 slip-stacked plus 6 regular batches, which increases proton intensity but has an insignificant influence on the final state energy of $120 \mathrm{GeV}$.

The current limit of 5 slip-stacked batches is a result of the rise and fall time of the injection magnets. Injection of a sixth slip-stacked batch would have the rise/fall of the magnets clip the beginning or the end of the adjacent batches in the MI, causing a radiation inducing spray of protons across the magnets and beam 


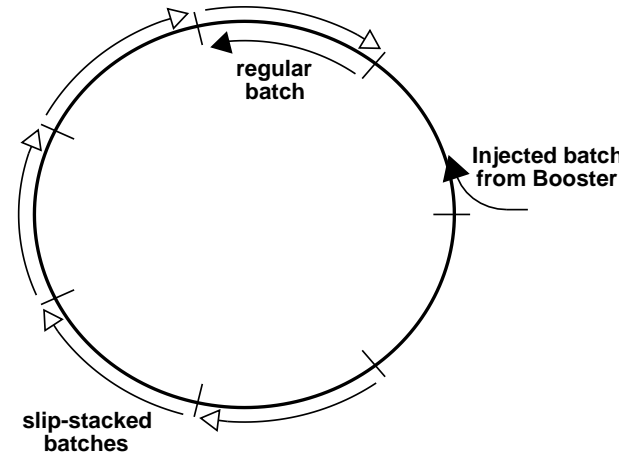

(a)
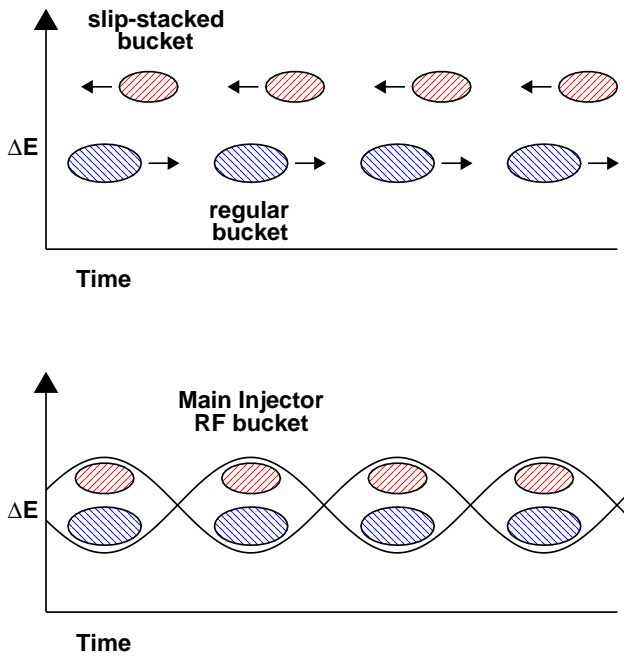

(b)

Figure 3.3: Cartoon of slip-stacking in the Main Injector. 5 slip-stacked batches are loaded into the MI in (a), while there is one orbiting regular batch and another being injected. The slip-stacked batches are slightly out of phase and slip back in the MI cycle until the buckets of the slip-stacked and regular are aligned in (b). Once the buckets are stacked the RF cavities capture the two smaller buckets into a larger MI bucket for acceleration to $120 \mathrm{GeV}$.

pipe. Ultimately, when the buckets from the slip-stacked and regular batches overlap, the RF cavities capture the protons into one of the 588 MI buckets ${ }^{1}$ (Booster buckets), see Fig. 3.3(b), Protons in the MI buckets are then accelerated to $120 \mathrm{GeV}$ for extraction to either the Tevatron anti-proton source or NuMI.

A significant limitation on proton intensity in the Main Injector results from protons injected from the Booster that rest outside the phase space of the MI buckets. These protons are left free to drift chaotically about the Main Injector ring, which has the consequence that some portion of the protons drift into areas of the MI cycle that should remain empty. When the magnets fire for injecting beam from the Booster to the Main Injector, any protons that are in the nominally free section of the Main Injector are deflected into the beam pipe and magnets. The localized radiation deposition sits at a prime access site in the Main Injector and limits the

\footnotetext{
${ }^{1}$ The 588 buckets correspond to the Main Injector being 7 times the circumference of the Booster, which itself contains 84 buckets.
} 
proton intensity to MINOS.

\subsection{NuMI Beamline}

While the accelerator complex provides the requisite protons, it is NuMI that converts the protons to neutrinos and focuses the intermediate hadron beam towards the Soudan Underground Mine.

The $120 \mathrm{GeV}$ protons from the MI are extracted in one $10 \mu$ s spill down the NuMI beamline, shown in Fig. 3.4. The proton beam is directed onto a thick carbon target, producing charged mesons, predominantly $\pi$ 's and $K$ 's, which decay to produce the desired muon neutrinos. The target consists of 47 segmented fins of carbon, each measuring $2 \mathrm{~cm}$ long by $6.4 \mathrm{~mm}$ wide by $1.8 \mathrm{~mm}$ tall. The carbon choice, type ZXF-5Q of Poco Graphite, was chosen for strength and resilience to high temperature. While each fin of the target is relatively thin, in total the target translates to two interaction lengths, which constitutes the designation as a "thick target".

Focusing the charged hadrons produced off the target is the only means to affect the direction of the neutrino beam and is achieved using two magnetic horns which are both parabolic in shape. While many of the secondary hadrons are focused, there is a small subset of high energy, low transverse momentum $\left(p_{t}\right)$ particles which pass through the middle of both horns and undergo no adjustment of direction.

The second of the parabolic horns, as well as the target, are movable in order to allow MINOS a flexibility in the neutrino energy spectrum. While data are taken between three different configurations (high, medium and low), the first MINOS physics results show the best sensitivity to oscillation parameters in the low energy configuration, LE10/185kA or LE010z185i; the different spectra are shown in Fig. 3.5. The LE010z185i configuration represents the target being set $10 \mathrm{~cm}$ into the 


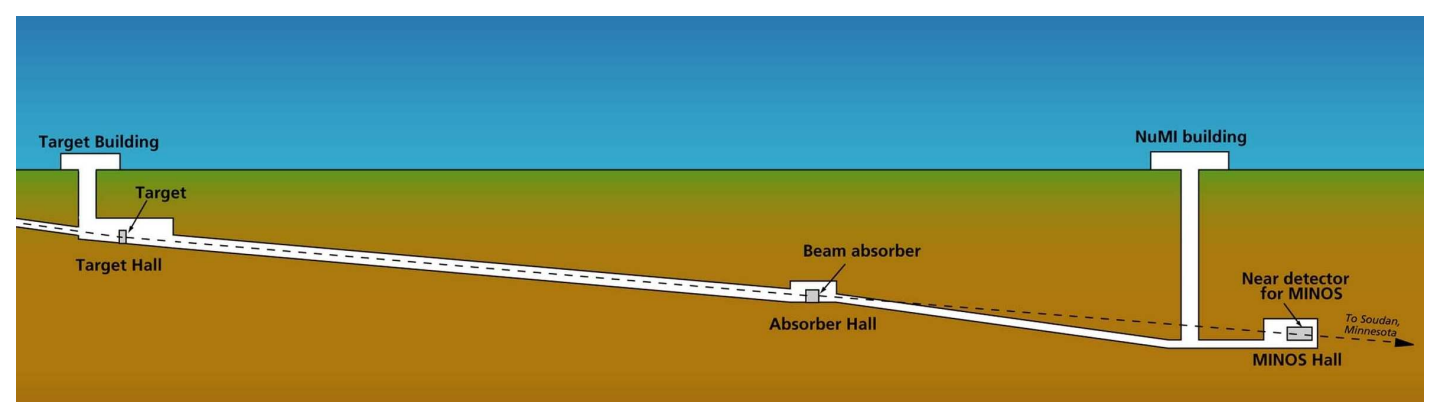

Figure 3.4: NuMI beam path, with the proton beam from the Main Injector incoming from the left. Protons from the Main Injector are directed $58 \mu \mathrm{rad}$ down towards Soudan, Minnesota.

most upstream horn while the magnetic horn pulses at $185 \mathrm{kA}$. The other configurations, varying in both target position and horn current, provide data useful for background and other systematic error studies. After passing through the horns the hadrons enter a $677 \mathrm{~m}$ evacuated decay pipe, where they decay into muons and muon neutrinos.

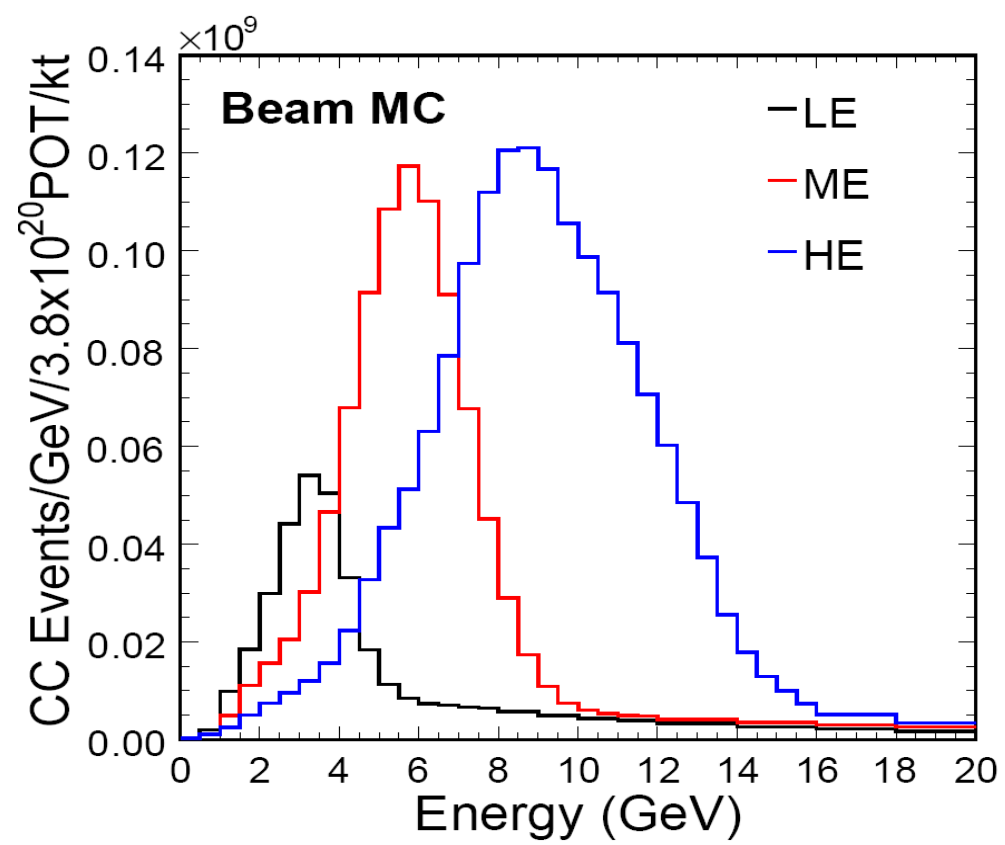

Figure 3.5: Monte Carlo energy spectrum of muon neutrino charged-current events in the Near Detector for three different beam configurations.

To limit contamination from decays that would occur beyond the decay pipe there is a hadron absorber located a few meters after the end of the decay pipe. The 


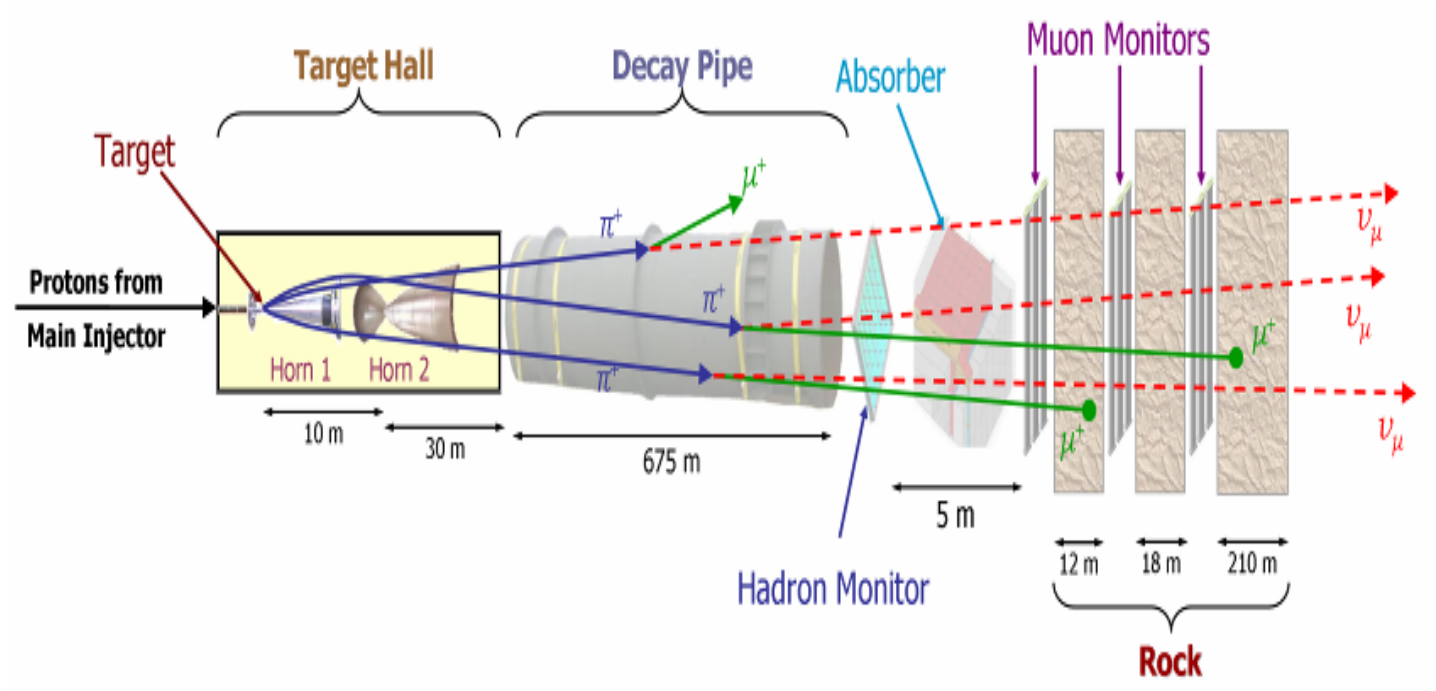

Figure 3.6: $120 \mathrm{GeV}$ Protons from the Main Injector are incident on the thick carbon target, where the ensuing hadrons are focused using two parabolic magnet horns. The hadrons then decay in an $\sim 677 \mathrm{~m}$ long decay pipe to produce neutrinos. Remnant hadrons are absorbed by the steel, aluminum and concrete hadron absorber, while muons and neutrinos continue on path. Three Muon Monitors downstream of the absorber detect muons to gauge direction and beam conditions correlated with the vertical/horizontal spread of the beam.

absorber has a core of water cooled steel and aluminum blocks which can withstand the heat and radiation from the remnant hadrons in the decay pipe. Real time analysis of the beam direction, height and width is done using three downstream muon monitors. While the main concern of the muon monitors is to observe the neutrino beam properties, measuring the muons produces a clear picture of the neutrinos that are produced in partnership. The center of the monitors are within $1 \mathrm{~cm}$ of the beam center and are formed of a 9x9 array of 3 in. $\mathrm{x} 3$ in. He ionization cells each spaced 10 in. apart from each other and shown in Fig. 3.7. The monitors have good spatial coverage and resolution of the muon beam features and have been used to diagnose beam related problems before they manifest in neutrino data.

Once the beam is focused and directed $58 \mu \mathrm{rad}$ down toward Soudan, the beam diverges from a couple of meters wide at the Near Detector to kilometers wide at the Far Detector. The divergence means that while the ND measures the whole con- 


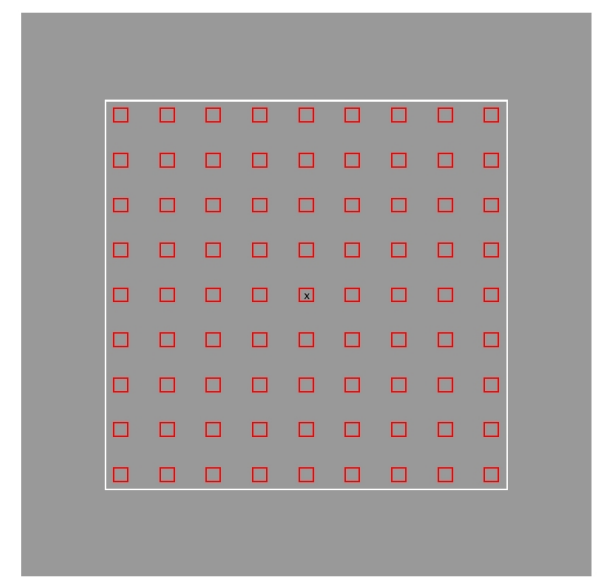

Figure 3.7: Muon Monitor from a Monte Carlo visualization. The white outline marks the housing of the monitor while each red box represents an individual $\mathrm{He}$ cell. The ' $x$ ' in the middle red cell marks the neutrino beam center, and illustrates the alignment with respect to the beam center.

stituent of the beam, the FD only measures the center of the beam. The kinematic spread of the beam core is given by

$$
E_{v} \approx \frac{\left(1-\frac{m_{\mu}^{2}}{M^{2}}\right) E}{1+\gamma^{2} \tan ^{2} \theta_{\nu}}
$$

where $m_{\mu}^{2}$ is the muon mass, $M$ is the parent hadron mass, $E$ is the parent hadron energy, $\gamma=E / M$ is the parent's Lorentz boost, and $\theta_{v}$ is the lab angle between the neutrino and parent hadrons directions. The difference in distance not only affects the energy of the neutrinos, but also the likelihood that a meson decay will produce a neutrino passing through the detectors. Decays occurring at the end of the decay pipe have a much higher probability to strike the Near Detector than the Far Detector. Differences related to neutrino energy spectra as well as solid angle acceptance between Near and Far Detector spectra are included in the Monte Carlo simulation when making energy spectrum predictions. 


\subsection{Hadron reweighting - SKZPTV}

The kinematics of secondary hadrons from the NuMI target directly affect the kinematics of the ensuing neutrinos. The resulting $\pi^{+}, \pi^{-}, K^{+}$, and $K^{-}$from the proton-target interactions are the particles that are focused by the magnetic horns and subsequently determine the flux at both the Near and Far Detectors. While there are a handful of models used to describe the resulting hadrons, BMPT and Sanford-Wang being chief amongst them, MINOS uses an intra-collaboration devised method named for the key contributors: Sacha Kopp, Žarko Pavlović and Tricia Vahle (SKZPTV).

The reason for creating a new model was that the NuMI neutrino beam resides in a region largely unexplored by previous hadron production measurements. As such, the models/parameterizations that were created and tuned using the previous data are not applicable to NuMI. For previous experiments the targets were often aluminum or beryllium, whereas NuMI uses carbon, and the incident proton beams were of energies either much lower than NuMI, 10-20 GeV/c [51, 52], or higher, > $200 \mathrm{GeV} / c[53$, 54]. The most significant difference is that most of the experiments used thin targets with an interaction length on the order of 1-5\% compared to the $\sim$ $200 \%$ interaction length NuMI target. Such a small interaction length significantly reduces any sensitivity to the effects of the secondary hadrons reinteracting within the target. For a full and complete discussion of accelerator neutrino beams see Ref. [55].

The importance of any hadron production model for a neutrino experiment is that the momentum of the secondary particles, specifically the longitudinal momentum, $p_{z}$, and transverse momentum, $p_{t}$, determine the energy of the neutrino, while the production angle determines the degree to which the neutrino is "focused" or pointed towards the detectors. The difficulty in making this prediction is illustrated in that previous experiments examining the neutrino flux found that their 
flux modeling, dependent on the secondary hadron production yields $d^{2} N / d p d \Omega$, was low by a factor of two[56] when compared with more exhaustive surveys[57] due to poor understanding of hadron production off of their respective targets. All subsequent neutrino experiments have struggled with the aspects of hadron production prediction and use different parameterizations and models to tune their Monte Carlo simulations. Creating a new model ensures that the hadron reweighting scheme is attentive to the unique aspects of the NuMI beam and ambitious goals of the MINOS experiment.

The SKZPTV uses the muon neutrino charged-current data from MINOS Near Detector, and in the future possibly muon monitor data, in order to reweight hadrons and obtain better Monte Carlo predictions ${ }^{2}$, Specifically the method smoothly warps the $\left(x_{f}, p_{t}\right)$ distribution, where $x_{f} \approx p_{z}$, of the secondary hadrons from Fluka2005. The distortion follows a loose parameterization of the BMPT [58] hadron production model, where the $p_{t}$ distribution for different slices of $p_{z}$ are fit using the following function:

$$
\frac{d^{2} N}{d x_{f} d p_{t}}=\left[A\left(x_{f}\right)+\left(B\left(x_{f}\right) p_{t}\right)\right] * e^{-C\left(x_{f}\right) p_{t}^{3 / 2}}
$$

where $A\left(x_{f}\right), B\left(x_{f}\right)$ and $C\left(x_{f}\right)$ are allowed to vary in the fit. The parameterization of $A\left(x_{f}\right), B\left(x_{f}\right)$ and $C\left(x_{f}\right)$ is established from fits that minimize the difference between Fluka2005 MC and Near Detector data from multiple beam configurations. The Near Detector data used in the fits have target offsets that range from $10-250 \mathrm{~cm}$ from the magnetic horn and horn current ranges from 0-200 kA. This covers a wide range of $\left(x_{f}, p_{t}\right)$ space and allows the SKZPTV method to disentangle the source of various systematic discrepancies. Ultimately each hadron in

\footnotetext{
${ }^{2}$ The charged-current, as opposed to the neutral-current, events are used because they provide the energy of the neutrino, which can be tied back to the parent hadron. Neutral-current neutrino events do not couple to the parent hadron kinematics as well as CC events because NC events have a visible energy over a range $0-E_{v}$.
} 


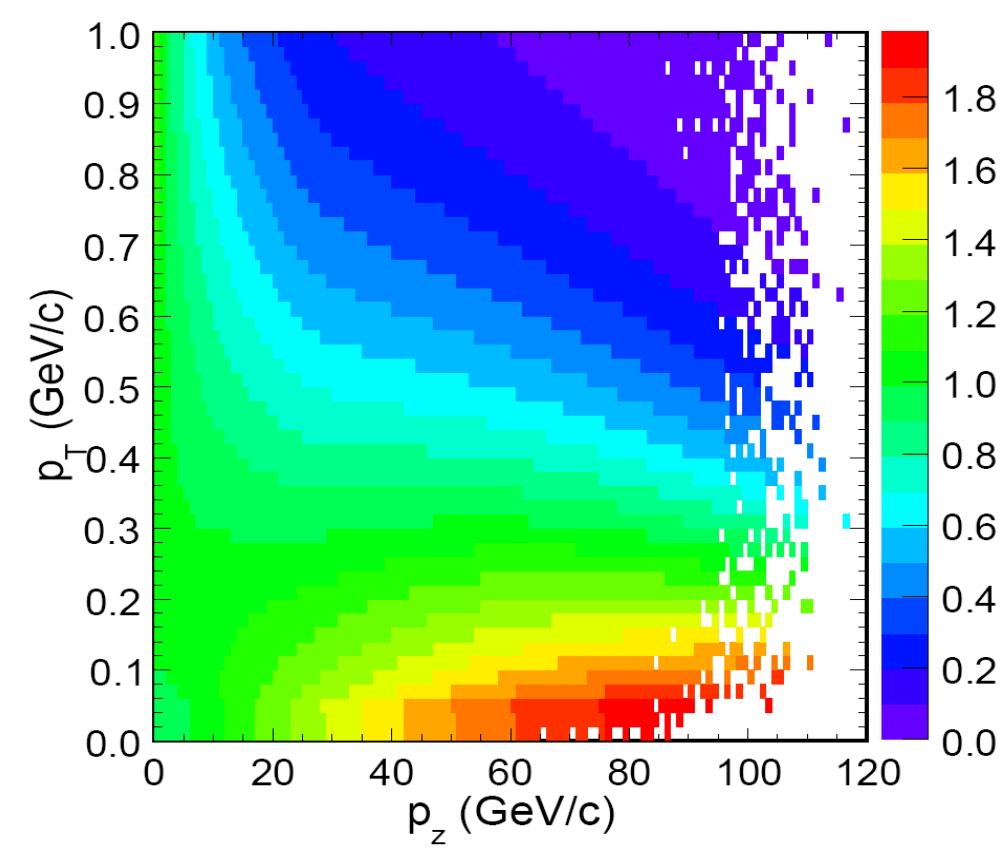

Figure 3.8: 2D histogram where the color, or z-axis, reflects the multiplicative scale factor applied to the MC neutrino parent hadron from the SKZPTV method. Determined as a function of $p_{t}$ vs. $p_{z}$, the weights from SKZPTV minimize data/Monte Carlo differences. As illustrated by the red region, corresponding to weights $>1.7$, the SKZPTV reweighting increases the contribution from the high energy tail.

the Monte Carlo is reweighted according to a combination of the parameters $A\left(x_{f}\right)$, $B\left(x_{f}\right)$ and $C\left(x_{f}\right)$. The weights applied to each hadron as a function of $p_{t}$ vs. $p_{z}$ is shown in Fig. 3.8.

While the SKZPTV method is used to distort the underlying $\left(x_{f}, p_{t}\right)$ of the neutrino parent hadrons, it also takes into account beam systematic uncertainties. Uncertainty in the horn current, target misalignment and electric current penetration within the skin of the horn are all aspects that affect the hadron production yield. The correction to the MC after application of the SKZPTV weights is shown in Fig. 3.9, where the MC/data agreement is shown to improve. The uncertainty related to the beam, and by proxy the SKZPTV reweighting, is the result of shifting all the uncertainties by $\pm 1 \sigma$, which creates $a+1 \sigma$ and $-1 \sigma$ error band around the nominal Monte Carlo prediction. 


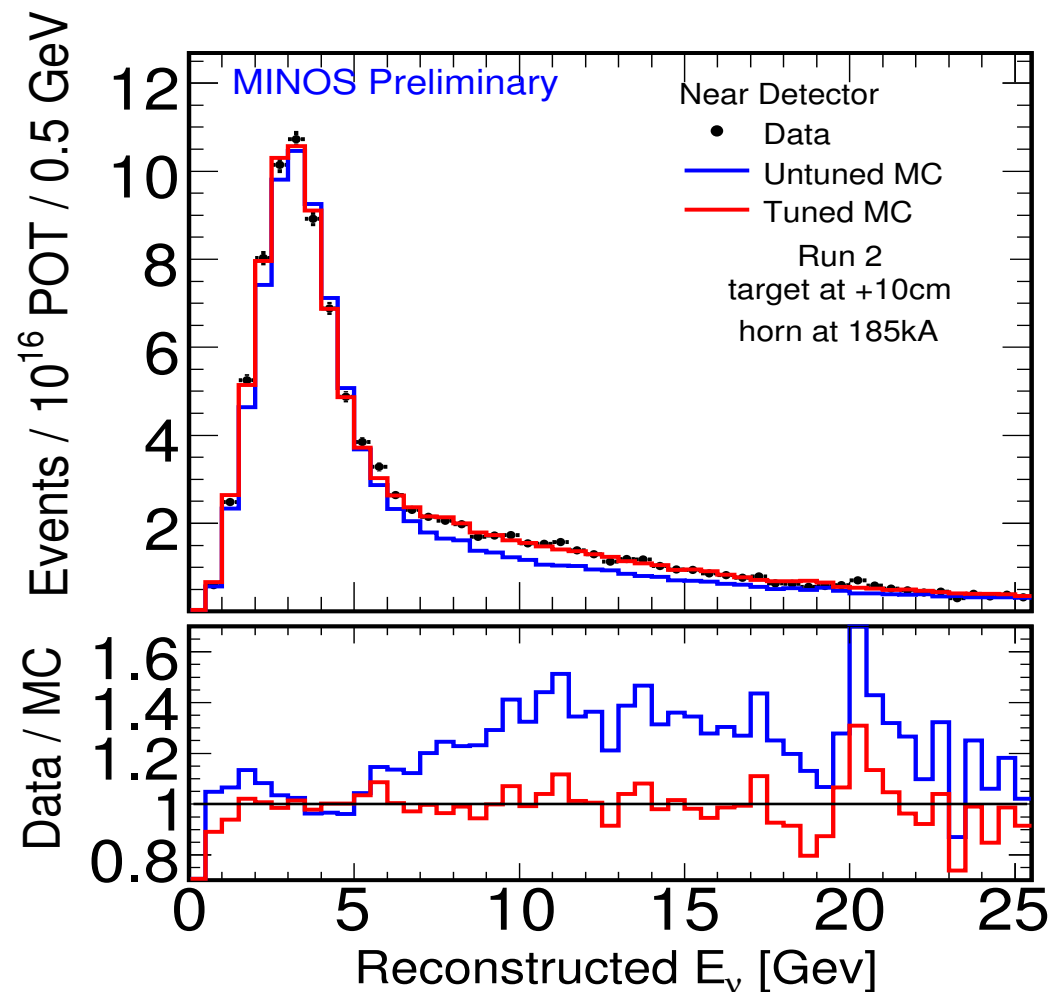

Figure 3.9: Near Detector charged-current energy spectrum for the RunII data set, before and after correction using the weights from SKZPTV. The Data/MC agreement over the $2-20 \mathrm{GeV} / \mathrm{c}$ region is greatly enhanced. 


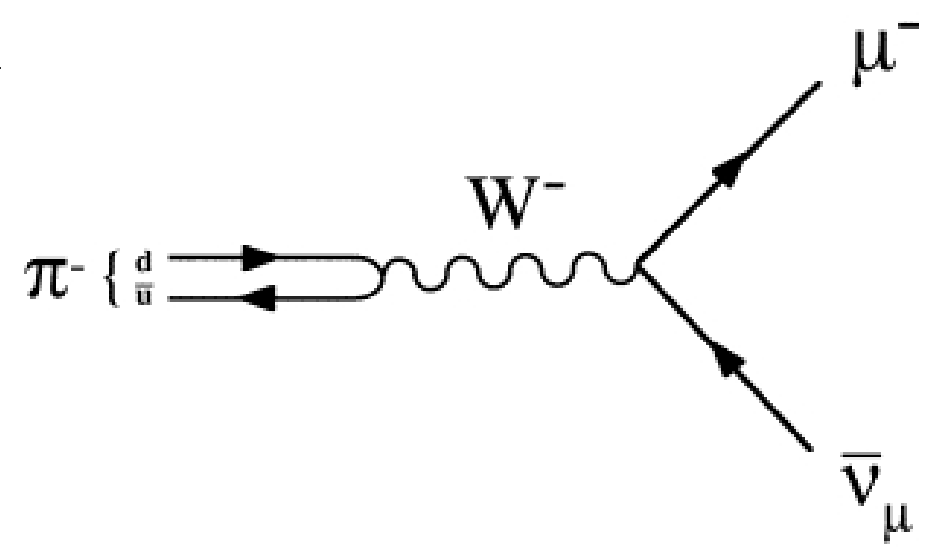

Figure 3.10: Feynman diagram of a pion decay producing a muon and muon neutrino in a one-to-one relationship. Both the muon and neutrino have the same parent hadron.

\subsection{Muon Monitors}

The main purpose of the three muon monitors is to quickly establish the NuMI neutrino beam intensity and direction. Beam issues or complications can be detected using muons on a pulse by pulse basis instead of waiting days or even weeks for the Near Detector to accumulate the necessary neutrino statistics. Beyond resolving the NuMI beam properties, the muon monitors can be used for physics analyses because of their ability to make direct measurements of muon intensity. Muons and neutrinos are produced in a one-to-one relationship from the same parent hadron, see Fig. 3.10, and any information gleaned about muon rate or the parent hadron applies to the neutrino. The difficulty in using these instruments for physics analyses lies in the fact they are not calorimetric devices, but instead simple sampling devices.

Each of the three muon monitors constitute a 9 × 9 array of helium gas ionization chambers; 9 streamer tubes each containing 9 cells (Fig. 3.11). The monitors record the amount of current caused by muons ionizing the helium, where the current is proportional, to first order, to the number of muons passing through the individual cells. As sampling devices, the monitors lack the ability to distinguish 


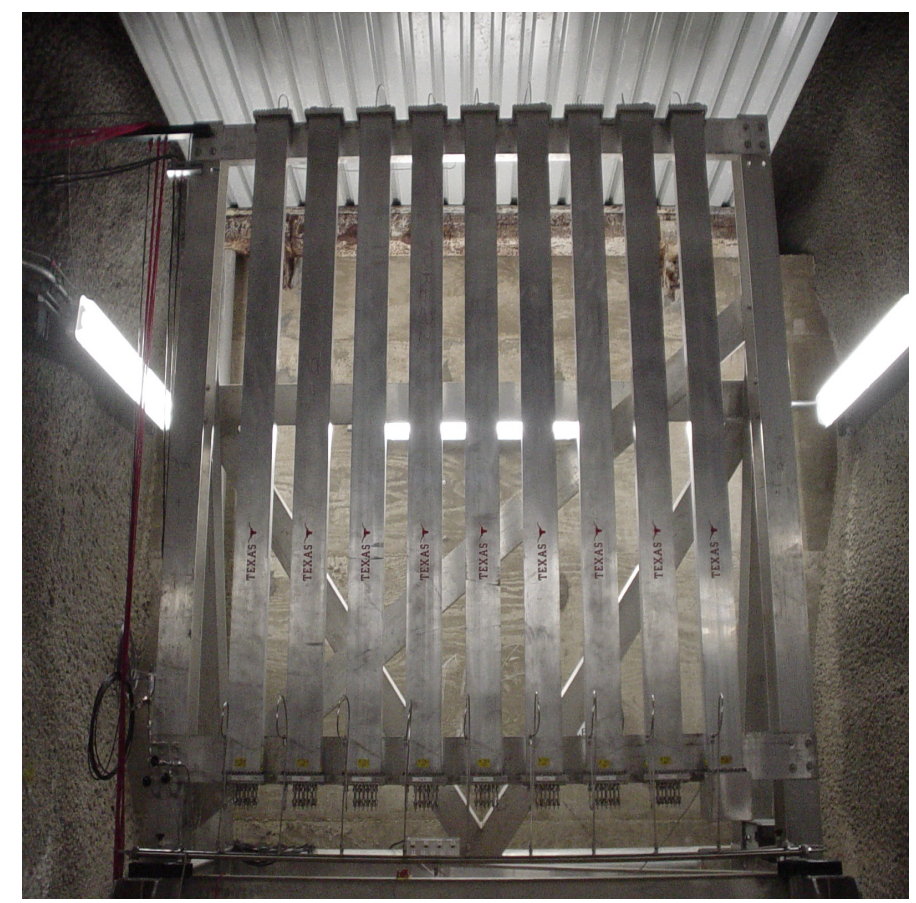

Figure 3.11: Photo of the nine streamer tubes that make up each muon monitor. Inside each tube are 9 ionization cells.

characteristics such as direction or energy from individual muons: an $8 \mathrm{GeV}$ muon will contribute as much to the muon monitor signal as a $0.5 \mathrm{GeV}$ muon. While the monitors cannot individually illuminate the energy of the muon beam, the varying amount of rock separating each monitor sets a momentum threshold required for a muon to reach the respective monitor (Fig. 3.12). With the data from the three monitors as well as from myriad of NuMI beam settings, muons can be used as direct data to do at least two important things for MINOS 1) estimate the muon/neutrino flux and 2) make further corrections to the underlying hadron production parameters. These analyses ultimately rely on an accurate MC prediction of the signal, which will be detailed in the following section.

Four different tools are used to make the prediction of the muon monitor signal: Fluka[59], GNuMI[60], a muon weighting function [61, 62], and G4NuMI. The tools have the following function:

I. Fluka - Hadron production off the NuMI target from incident $120 \mathrm{GeV}$ pro- 


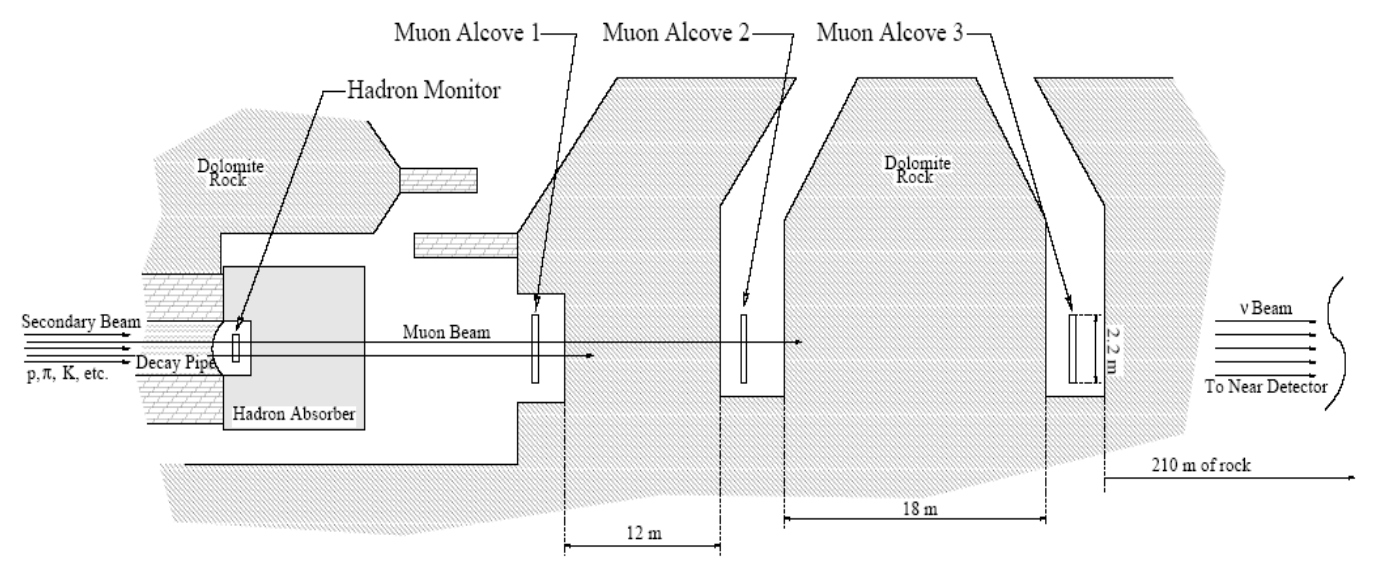

Figure 3.12: Location of each muon monitor from an above view. Illustrated is the approximate amount of rock that separates the various muon monitors. The rock increases the amount of energy of a required to reach each successive monitor.

tons

II. GNuMI - Geant3 Monte Carlo used to transport the hadrons from the NuMI target through the focusing horns to their decay point and neutrino creation.

III. Weighting Function - Provides the likelihood of muon produced in the hadron decay to propagate to the end of the decay pipe.

IV. G4NuMI - Geant4 transport simulation of muons from the end of the decay pipe through the Hadron Absorber and rock to the muon monitors.

Of the four tools used to make a prediction of the signal at the muon monitors, the least developed aspect was the integration of the NuMI beamline and muon propagation downstream of the decay pipe with the Geant4 Monte Carlo. The importance of this portion of the Monte Carlo chain is that the behavior (and, more importantly, energy loss) of muons between the end of the decay pipe (EODP) and the monitors is heavily influenced by the location and density of the objects that the muons traverse. A source of difficulty in reconstructing every possible object a muon may traverse was that many of the records, engineering drawings and 


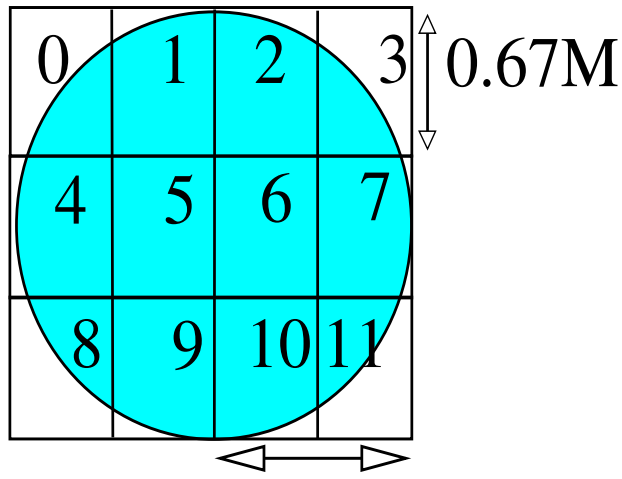

$1 \mathrm{M}$

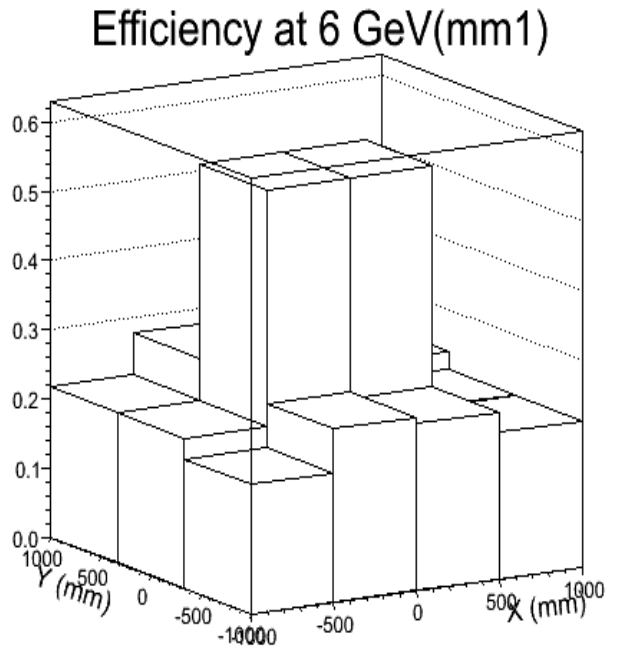

Figure 3.13: The $4 \times 3$ grid that the the muons at the end of the decay pipe are split into in order to account for differences in material. The figure on the left shows the end of the decay pipe in blue with the lines of delineation separating the different regions of common efficiency. The figure on the right shows the difference in efficiency for muons at the first muon monitor which pass through the core of the Hadron Absorber versus those that traverse the shielding.

invoices were scattered like leaves in the wind thereby arresting scientific progress in favor of a clerical scavenger hunt.

After a complete accounting and encoding of the location, density and chemical composition of known objects residing between the EODP and the muon monitors [63] the G4NuMI MC can be used to find the likelihood of a muon at the end of the decay pipe to reach the muon monitors. Instead of doing this with each individual muon, the G4NuMI was used to create efficiency curves of a muon to reach each muon alcove as a function of momentum. The efficiency is calculated as

$$
\varepsilon\left(E_{\mu}\right)_{i=1,2,3}=\frac{\sum \mu_{i}}{\sum \mu_{E O D P}}
$$

where $\varepsilon$ is the efficiency at the three different monitors, $E_{\mu}$ is the energy of the muon, $\mu_{i}$ is the number of muons reaching the muon monitor $i$, and $\mu_{E O D P}$ is the number of initial muons at the end of the decay pipe. The muon efficiency is 


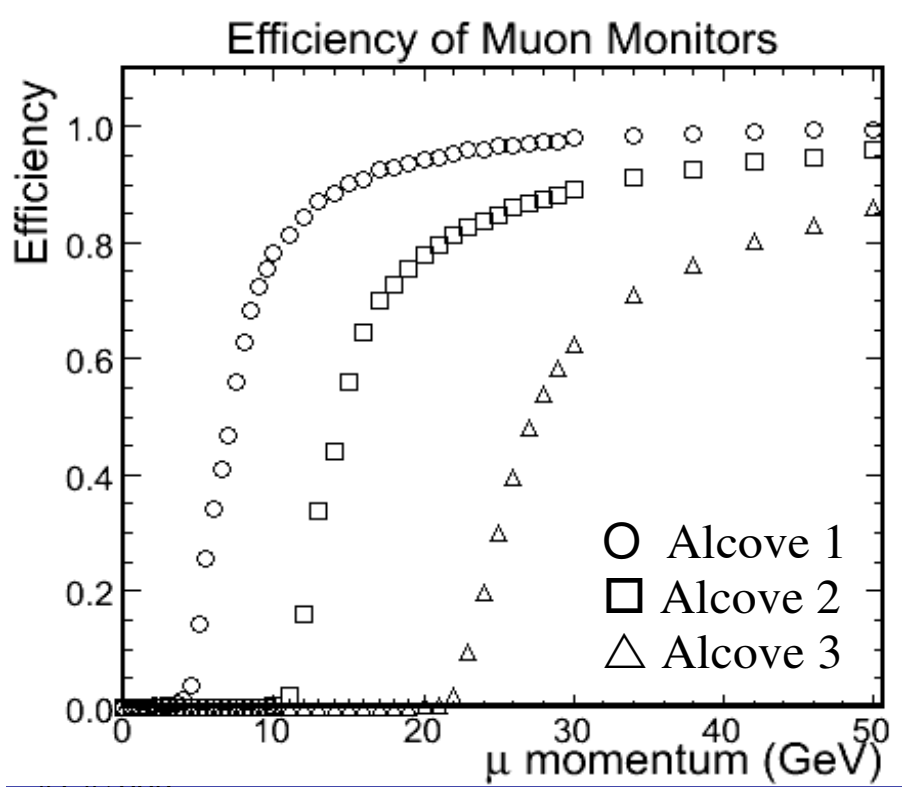

Figure 3.14: Efficiency curves for a muon to reach the three alcoves. Each subsequent downstream monitor has an increase in the minimum momentum required in order to reach the alcove. The figure is for only one of the six sections that make up the end of the decay pipe.

calculated for 6 different sections of the end of the decay pipe, because the slight downward slope of the NuMI beam as well as the different density regions of the Hadron Absorber make the efficiency of a muon to reach the muon monitors not only energy dependent but position dependent as well. The dependency on location is illustrated in the right plot of Fig. 3.13, where a uniformly distributed beam of muons at $6 \mathrm{GeV}$ shows that muons exiting the Decay Pipe in the center have a much higher likelihood of reaching the first muon monitor. The efficiency curves over all the energies are shown in Fig. 3.14

With the efficiency curves in hand, the momentum distribution at the muon monitors can be established by multiplying the efficiency by the muon EODP momentum distribution, which is shown in Fig. 3.15. The predicted spectrum, shown in Fig. 3.16, shows that there is a dramatic decrease in the number of muons that reach each successive downstream monitor, which is the effect of the rock that separates them. 


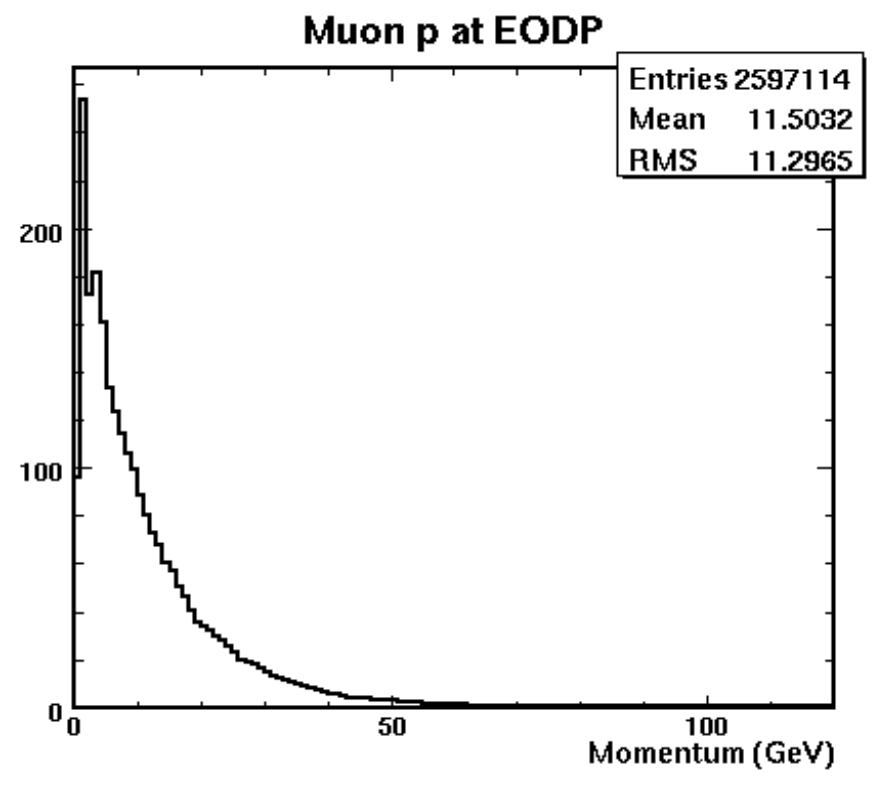

Figure 3.15: Histogram of the muon momentum at the end of the decay pipe for the L000z010i target position.

The last step to making the prediction is to convert the MC momentum spectrum at the monitors into a signal that is comparable to the data, where the signal from the muon monitors, being made up of ionization cells, is proportional to the integrated energy deposition of particles that pass through each cell. Muons have a flat energy deposition in the momentum range of muons from the NuMI beam, 1-120GeV. Subsequently, a $90 \mathrm{GeV}$ muon and a $5 \mathrm{GeV}$ will deposit approximately the same amount of energy into the muon monitor, and the predicted signal of the muon monitors is then the integration of the muon momentum distribution at the alcoves shown in Fig. 3.16. Reproducing the total process for each beam configuration gives a range of predictions that can be plotted to show the predicted response as a function of horn current and target position (Fig 3.17(a)).

A qualitative comparison between the predicted muon monitor and actual muon monitor signals in Fig. 3.17 show significant similarities. Some of the important features are

- There is a roll-over in the le250 target position when the focussing horn is 
Muon Dist. Alcoves (le000z010i, Nominal)

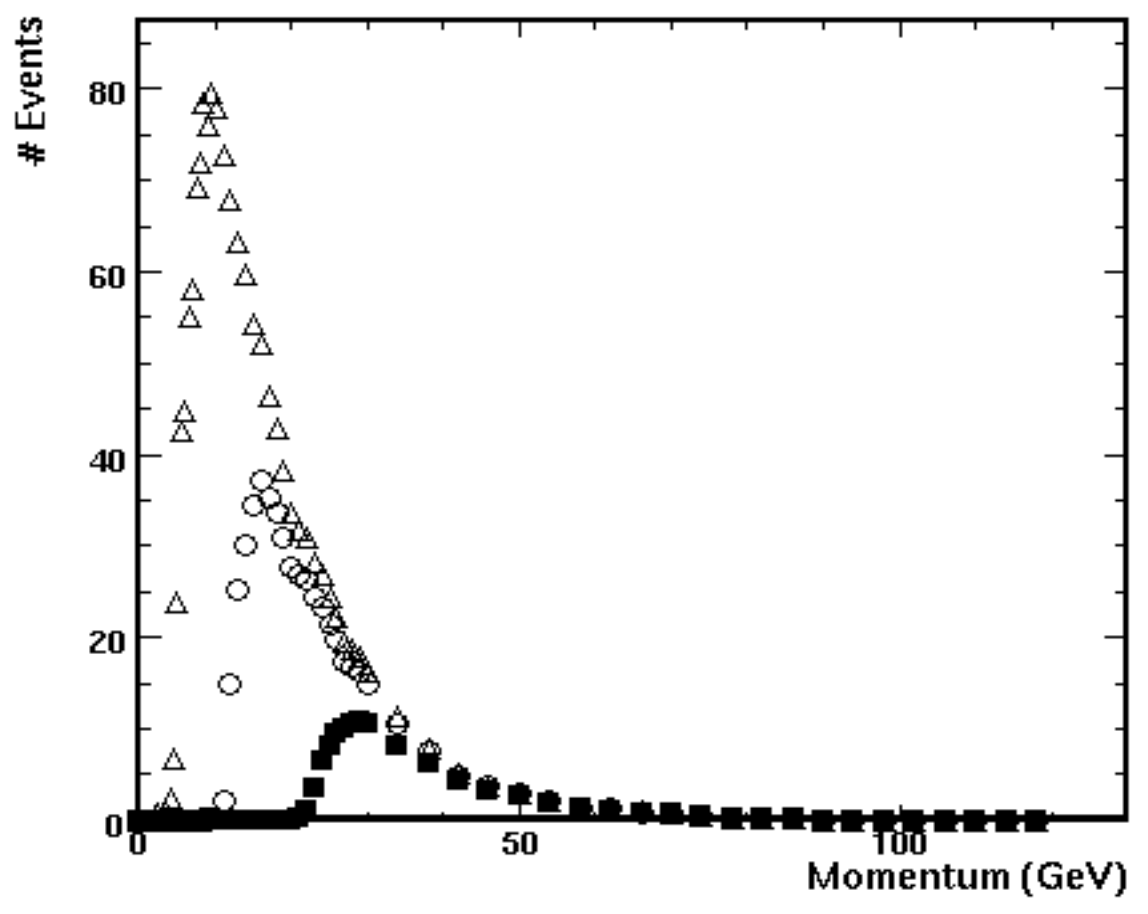

Figure 3.16: Predicted distribution of muons at the muon monitors for the LE000z010i beam. Triangles mark the muon distribution at the first alcove, circles the second, and closed squares the third. The distributions are the combined product of all 6 segregated sections that make up the end of the decay pipe. The decrease in efficiency between each monitor is the effect of the rock in between each muon alcove. 

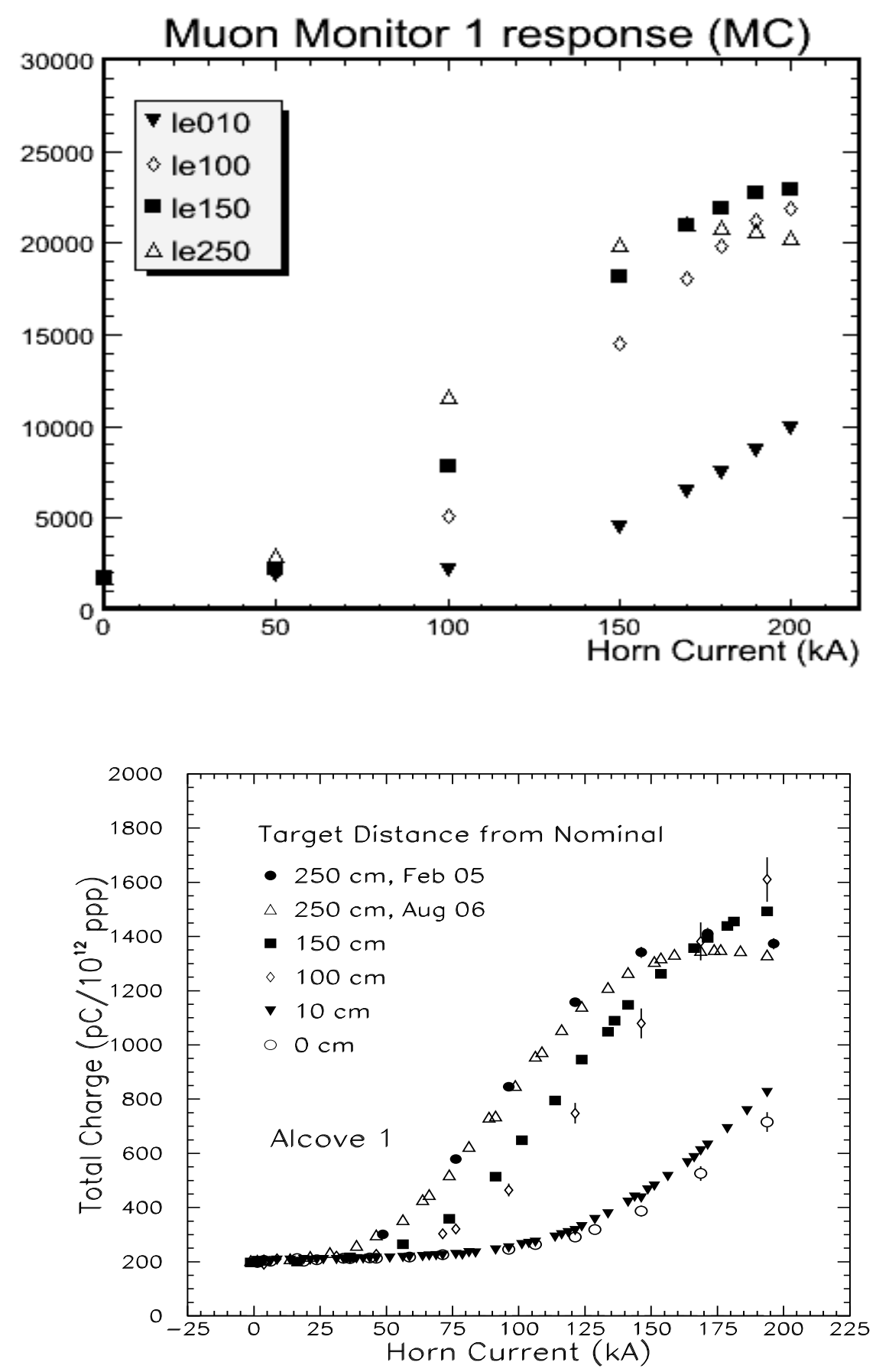

Figure 3.17: MC predicted signal(top) as well as actual data(bottom) at the first muon monitor for various beam settings. The values on the vertical abcissa of the MC prediction are quantitatively irrelevant. A future quantitative comparison between prediction and data will incorporate a normalization scheme. 
pulsing at a current of $\sim 160 \mathrm{kA}$. Settings greater than $160 \mathrm{kA}$ begin to overfocus the parent hadrons of the muons, and neutrinos, and decrease the flux.

- The separation between signal points has the same relative difference between beam settings.

The final conclusion is that the software tools have been put in place in order to make a MC comparison to data from the muon monitor signal. The prediction, without any tuning or significant modification, parallels many of the nuanced features of the data. The possible physics analyses available because of this development are a direct flux calculation and further modification to hadron production modeling. 


\section{Chapter 4}

\section{The Detectors}

The MINOS experiment consists of three detectors: the Far Detector, Near Detector and Calibration Detector. The Near Detector is located at Fermilab in Batavia, Illinois and is situated $\sim 1 \mathrm{~km}$ downstream of the NuMI target. The Far Detector is situated $735 \mathrm{~km}$ from the target in the Soudan Underground mine in northern Minnesota. The Calibration Detector resided at CERN in Geneva, Switzerland, until its dismantling in January 2004. The design of the detectors was exploited to detect particle interactions and use curvature in a magnetic field to separate $v_{\mu}$ from $\bar{v}_{\mu}$ in the Near and Far Detector.

Although the detectors are intrinsically similar in their design, the Near, Far and Calibration Detectors' purposes and construction are subtly different. The differences are related to size and electronics, while the actual construction material of the detectors, notably steel and scintillator, is the same for all three.

\subsection{Collective properties}

The detectors are constructed of 1 inch thick steel planes followed by active scintillator attached to each steel plane. Each 'sandwiched' layer of steel and scintillator is separated by an air gap necessitated by construction. The type of steel chosen, 
American Institute of Steel and Iron (AISI) 1006, maintains the tensile strength required to avoid fatigue and warping while also ensuring sufficient magnetic permeability. The selection was also made on the basis of ease of welding, as steel planes in the Far Detector were welded together out of an assortment of smaller steel plates and the scintillator planes would also be welded to the steel. Near Detector steel planes were fabricated from single 1 inch thick steel plates.

The scintillant used in MINOS is polystyrene doped with $1 \%$ PPO fluor, and $0.030 \%$ wavelength shifter POPOP[64]. The scintillant is housed in strips which measure $1 \mathrm{~cm}$ by $4.1 \mathrm{~cm}$, co-extruded and ensconced in an outer layer of $\mathrm{TiO}_{2}$, which acts as a reflective layer to keep photons created in the scintillator contained in the strip. The light from the scintillant is absorbed by Kurray Y-11 fluor Wavelength-Shifting (WLS) fibers, epoxied into a groove in the middle of the scintillator strips, Fig. 4.1. The WLS fibers run the length of the strips, with a small amount protruding from the end.

The WLS fiber absorbs the 420nm light emitted by the scintillator and shifts it to a lower wavelength to minimize attenuation. With an absorption spectrum centered on 420nm, and an emission spectrum centered at 470nm, the WLS fibers not only capture light from the scintillator and decrease attenuation but also minimize self-absorption. The light ultimately illuminates a Hamamatsu R5900 multi-anode class Photomultiplier Tube (PMT), used in both Near and Far Detectors, which has a peak wavelength efficiency at $420 \mathrm{~nm}$ with a spectral response from 300$650 \mathrm{~nm}[65][66]$.

The scintillator strips are grouped side-by-side into modules, which are arranged in bunches of either 16, 20, 24 or 28 scintillator strips per module. Each module is encased in an aluminum skin to suppress ambient light as well as protect the flammable scintillant from sources of ignition. The edges of each module are mounted with manifolds that connect the WLS fibers in each scintillator strip 


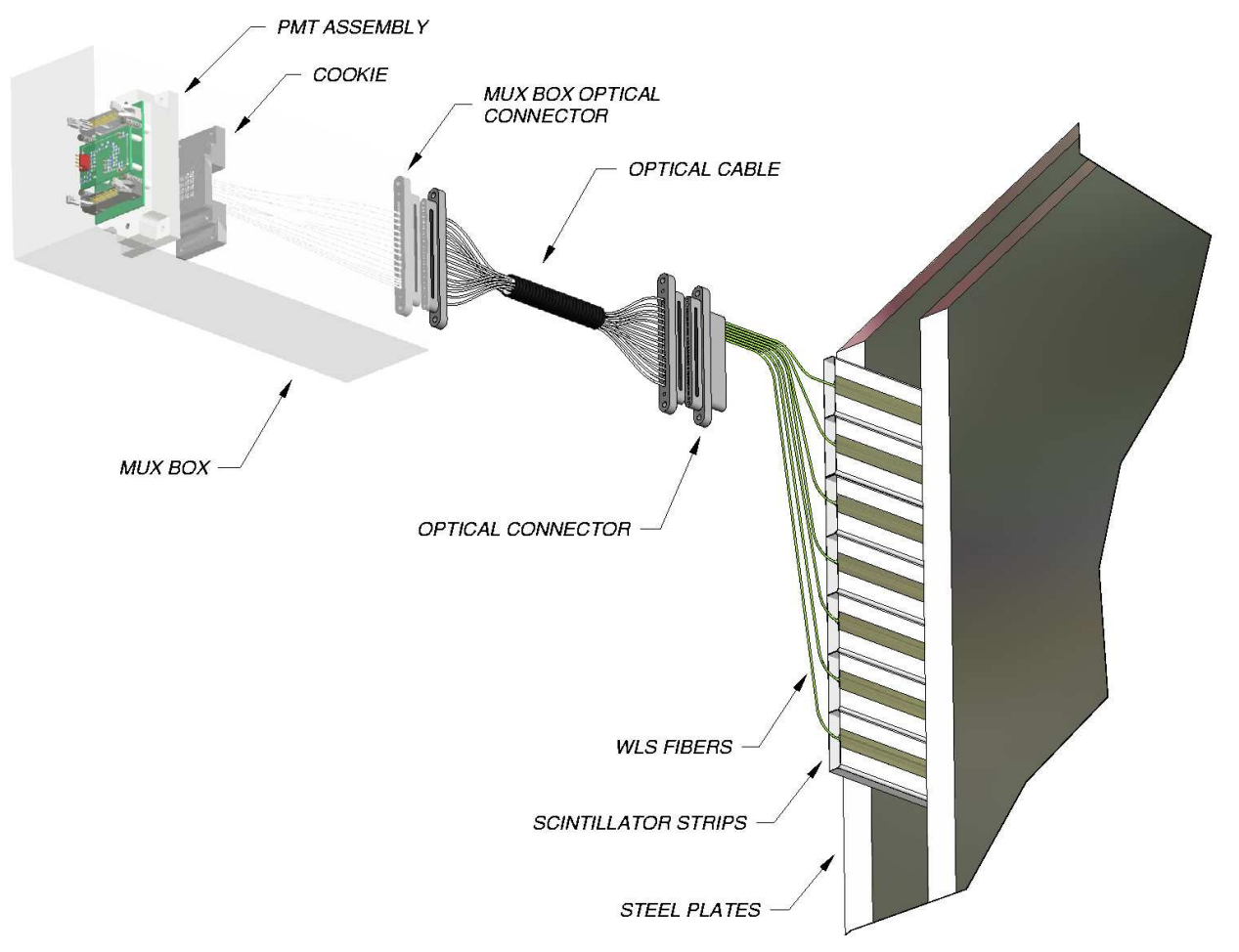

Figure 4.1: This layout shows the connections made by the optical fibers for the detectors. 


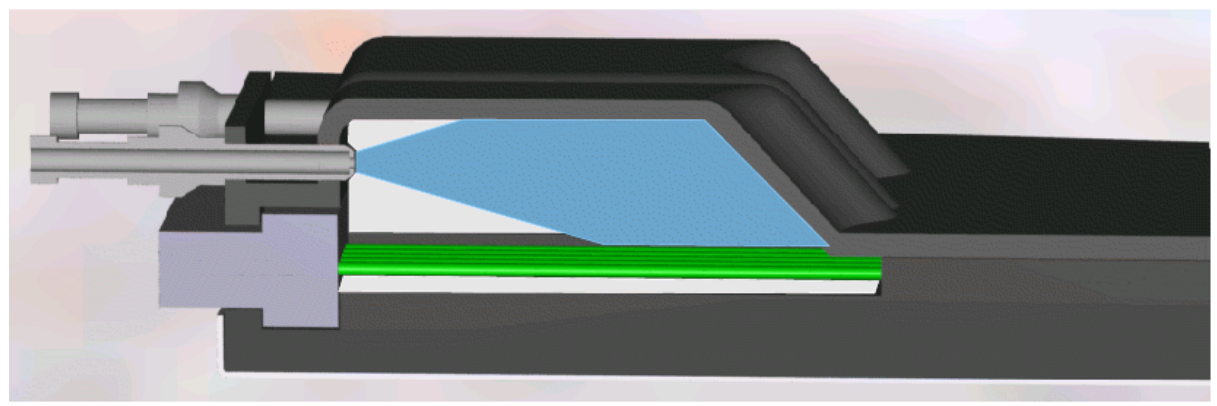

Figure 4.2: Cross section of the scintillator module manifold connecting the WLS to clear optical fiber as well as the light injection module mounted on the manifold.

to clear optical fiber cables running to a PMT. The fiber transition further reduces attenuation. In conjunction with attaching WLS fiber to clear fiber, each manifold also attaches to 2-3 optical cables from the Light Injection (LI) system that pulse, or flash, directly onto the grouped WLS fibers for calibration, see Fig. 4.2. Scintillator modules are grouped into planes, with each plane rotated $90^{\circ}$ to the preceding one in order to provide 3-dimensional tracking.

\subsection{Calibration}

To ensure that the optical readout of the detectors is working properly, a few calibration checks have been constructed. The main calibration tools used are the Light Injection (LI) system, cosmic ray muons and a mapper using a radioactive source. As the response of the detector changes, due to changed electronics, temperature changes, scintillator aging, etc..., these tools are used to either identify problems or to monitor/correct drifts of detector response over time. The total calibration chain that produces a calibrated charge, $Q_{\text {calib }}$, from the raw charge, $Q_{\text {raw }}$, can be characterized by a linear combination of calibration processes:

$$
Q_{\text {calib }}=Q_{\text {raw }} \times D(t) \times L\left(s, Q_{\text {raw }}\right) \times S(s, t) \times A(s, x) \times E
$$


where $t$ represents time, $s$ the scintillator strip hit and $x$ the position of the hit along the scintillator strip. The following sections will cover each of the intradetector calibrations constants ( $D$ - Drift, L - Linearity, $S$ - Strip-to-strip and A - Attenuation), while $E$, the absolute energy scale of each detector, is established using stopping muons.

\subsubsection{Light Injection}

The LI system is designed to test the optical readout response to blue light, similar to the scintillator emission, from a controlled source pulsed directly onto the WLS fibers. The light is supplied by 'pulser' boxes, which contain 20 Hewlett-Packard HLMPCB30 wide angle LEDs[67]. The pulser box flashes the LEDs, normally 1000 times, with linearly increasing pulse heights. Each LED illuminates a bundle of fibers, where most are routed through the rear of the box and attached to a light injection module mounted on each scintillator module manifold: 2-3 LI fibers per module. To independently verify that the LEDs are producing a linearly increasing pulse height, a subset of the LI optical fibers illuminate a group of PIN photodiodes. The aim of the LI system is to

- $D(t)$ - Monitor short-term drifts in the gain of the PMT. PMT response is very sensitive to changes in temperature as well as applied high voltage. The LI system monitors any short-term drifts in response by pulsing hourly at a constant pulse height and then comparing the PMT/PIN response to a reference point. There is also a long-term component to the $D(t)$ constant which is covered in Sec. 4.2.2.

- $L\left(s, Q_{\text {raw }}\right)$ - Map the gain curve of the PMT. The response of a PMT is inherently non-linear at high light levels, as well as different between individual PMTs. Measuring the response to a linear pulse height affords a conversion between PMT response and the amount of light 'seen' in the scintillator. 
- Determine location of single photoelectron peak. In order to use Poisson statistics for low-light events, the SPE peak must be known.

\subsubsection{Cosmic Rays}

Cosmic ray muons are the dominant tools by which MINOS calibrates the detectors. Through-going muons are used to measure the strip-to-strip variations as well as the drift in response over time.

The strip-to-strip calibration, $S(s, t)$ in the calibration chain allows for varying responses between strips to be taken into account. While the LI system is useful for characterization of PMT and electronic response, it does not account for differences related to WLS fibers or scintillant within the scintillator strips, where WLS fiber length constitutes the largest cause of strip-to-strip variations. Muons, being minimum ionizing particles, deposit a similar amount of energy in each plane traversed, and owing to their natural abundancy, make useful tools to examine intra-detector strip differences. Using cosmic ray muons the strip-to-strip calibration is calculated using the mean of the muon spectrum measured in arbitrary Minos Eenergy Units (MEU) compared to the mean response of every other strip in the detector.

$$
S(s, t)=\frac{\text { Mean Response of Detector }}{\text { Mean Response of the Strip End }}
$$

While the calibration uses the mean of the muon spectrum, corrections are done on an event-by-event basis in order to compensate for path length differences as well as statistical fluctuations relating to low light-levels.

Besides the short-term drift, there is also a long-term drift that is mainly related to scintillator aging. The light output of scintillator decreases over time at the rate of $\sim 2 \%$ per year, shown in Fig. 4.3. With a high amount of muon statistics the aging can be tracked and corrected over time. 

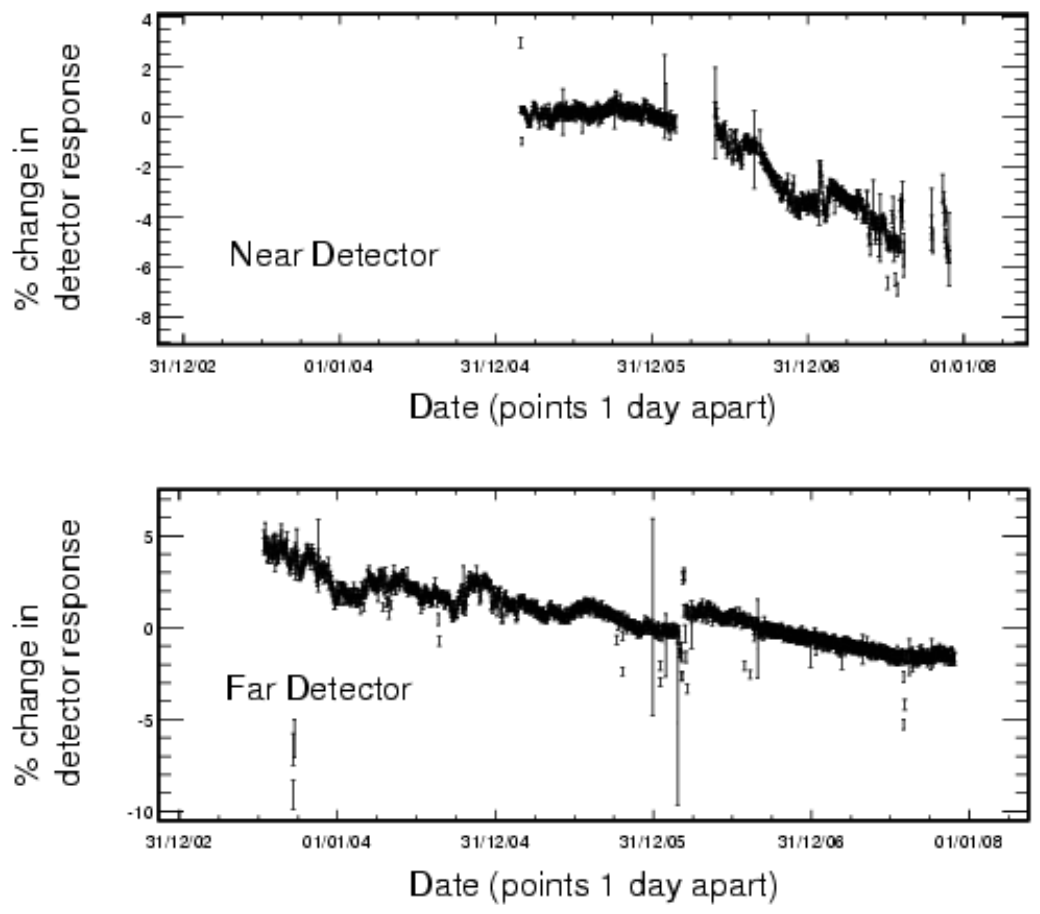

Figure 4.3: Drift in response for both the Near(top) and Far(bottom) Detector. The gap in the Near Detector represents the 2006 shutdown. The points represent percentage drift in one days data from an arbitrary zero point, where the error bars are the rms of the daily pulse heights per plane.

The absolute energy calibration, $E$, of each detector is done using a large sample of stopping muons. The calibration examines the detector response in the $0.5 \mathrm{GeV}$ - $1.1 \mathrm{GeV}$ energy region of a muon track; a region avoiding the rapid variation in energy deposition, $\mathrm{d} E / \mathrm{d} x$, at the end of the track. The mean response is calculated for each strip and the median for all strips in the detector represents the single energy constant.

\subsubsection{Mapper}

The light response as a function of location on each scintillator module was mapped upon delivery at either the Near or Far Detector with a radioactive source. The source, Cesium 137, produces $660 \mathrm{KeV}$ gamma rays which are used to provide a baseline response of individual strips in the module. The data from the mapper 
were used to provide the attenuation constant $A(s, x)$, via an empirical model of attenuation for both the WLS as well as the clear optical fiber.

$$
A(s, x)=A_{1} e^{-x / L_{1}}+A_{2} e^{-x / L_{2}}
$$

where $x$ is the length along the strip and $L_{1}, L_{2}$ stand for the two attenuation lengths corresponding to the East and West side of the module.

\subsection{Magnet}

Each detector contains a current carrying magnet coil that provides a toroidal magnetic field with a strength of $\sim 1.2$ Tesla in the respective regions of interest. The field allows MINOS the opportunity to distinguish between $v_{\mu}$ and $\bar{v}_{\mu}$ interactions as well as calculate muon momentum from track curvature. A topological map of the magnetic field strengths for both detectors is shown in Fig. 4.4. The maps are produced using a Finite Element Analysis (FEA) utilizing data from probe measurements embedded in a MINOS plane prototype. A secondary check is an event by event comparison of stopping muon momentum.

The accuracy of the field maps from the FEA analysis is checked by using the muon momentum from track curvature. Momentum of a stopping muon can be calculated using two redundant methods: range $\left(P_{\text {range }}\right)$ and curvature $\left(P_{\text {curvature }}\right)$, where calculations from $P_{\text {range }}$ have an uncertainty of $2 \%$. The calculation using $P_{\text {range }}$ when compared to $P_{\text {curvature }}$ via a double ratio of $\mathrm{MC}$ and data for $P_{\text {range }}$ $P_{\text {curvature }}$ gives the means to assess the magnetic field uncertainty in the detector(s). The double ratio yields:

$$
\left(P_{\text {range }} / P_{\text {curvature }}\right)_{\text {data }} /\left(P_{\text {range }} / P_{\text {curvature }}\right)_{M C} \approx 1.01
$$

The consistency of the magnetic field is maintained by the BDot system, which 


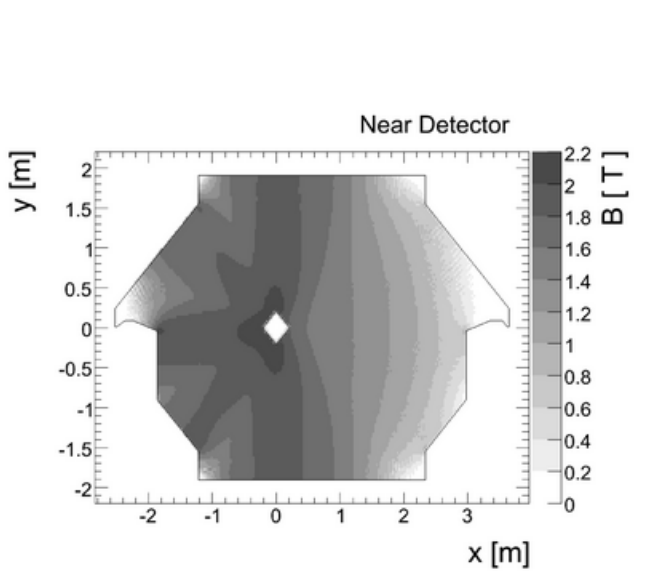

(a)

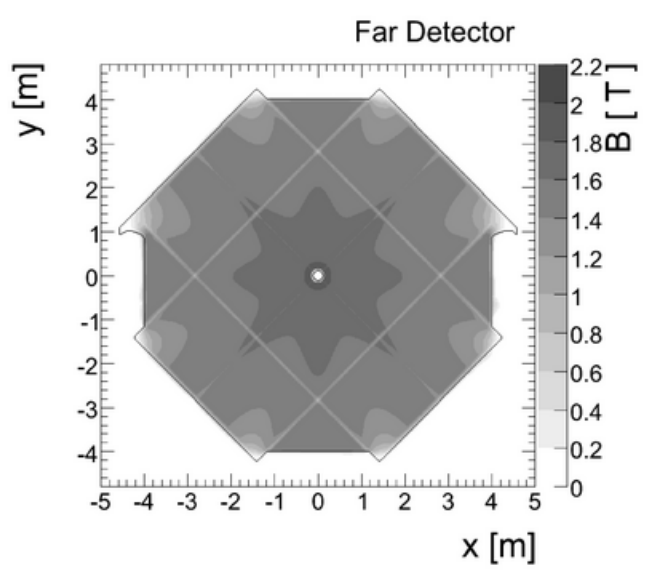

(b)

Figure 4.4: Magnetic field maps calculated from Finite Element Analysis. Plot (a) shows the Near Detector plane, while (b) shows the Far Detector. Clearly illustrated is the effect of the welding joints between steel plates in (b).

is used to reliably set the strength of the magnetic field when reversing the magnetic field or power cessation, whether deliberate or unanticipated, to the magnetic coil. This is not a routine maintenance procedure but a process performed only when the magnet is brought back to full field strength. The BDot system consists of

- BDot cables. Strung along the steel planes, the 50 wires in the BDot ribbon cables transmit an induced current in the presence of a changing magnetic field. Since the cables are attached to the steel planes, the current they produce is directly related to the changing magnetization of the steel immediately under the cable.

- Keithley card. 32 BDot cables attach to a single Keithley card which processes the Analog to Digital Conversion (ADC) and relays the digitized induced current data to a computer.

- BDot computers. A LabView software program on each slave BDot computer is used to monitor the current readings from the BDot response cables, while a similar LabView program on the master BDot computer changes the 


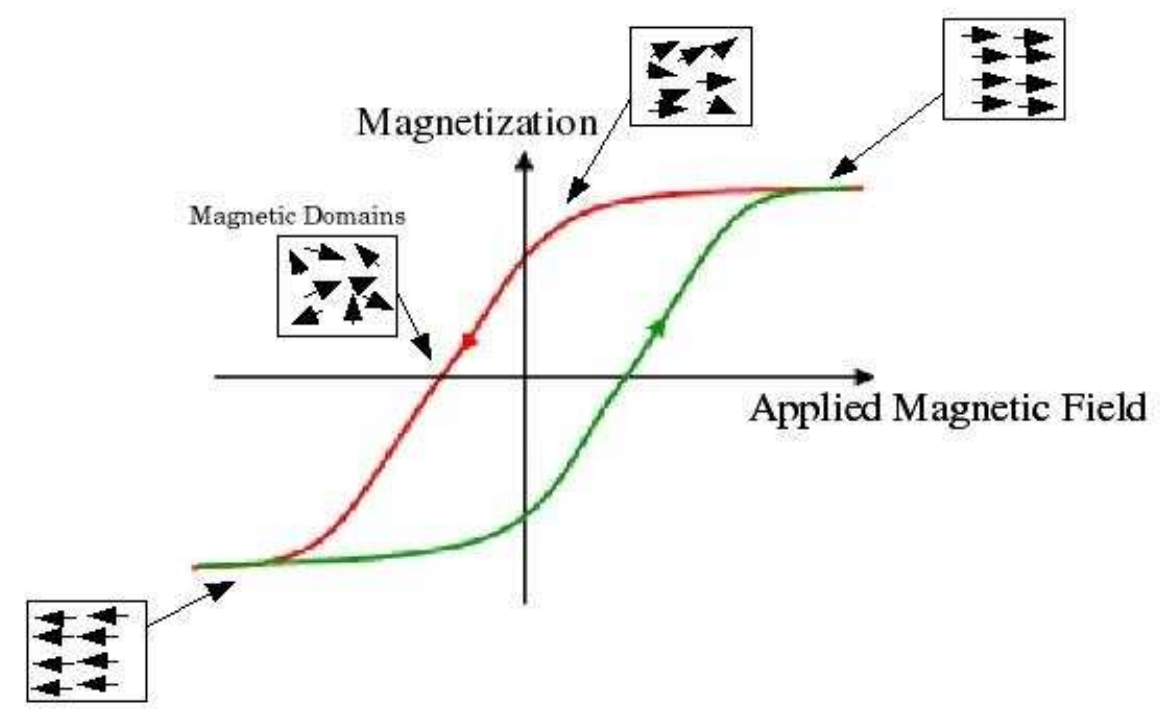

Figure 4.5: Ideal hysteresis loop showing the orientation of magnetic domains at varying imposed magnetic field strengths. The end points represent saturation where all the magnetic domains are oriented in the same direction and magnetic field strength is uniformally maximum.

input current for the magnet coil power supply.

- National Instrument FieldPoint. Relays commands from the BDot computers to the magnetic coil power supply.

When changing the magnetic field of ferromagnetic materials, in this case steel, the exercise ideally follows a hysteresis loop, which illustrates the relationship between applied magnetic field and magnetization of the steel (Fig. 4.5). The goal is to uniformally orient the magnetic moments in a similar direction, which in turn produces a uniform magnetic field across the entire face of the steel planes matching the magnetic field maps. Executing the cycle too quickly results in a random assortment of unaligned magnetic moments, leaving localized areas of the steel with unknown magnetic field strengths. Thankfully, previous analysis has automated and optimized the ramp cycle to ensure consistent magnetic field[68] 


\subsection{Near Detector}

The main purpose of the Near Detector is to measure the unoscillated neutrino spectrum. A tertiary use of the detector is as a diagnostic tool to identify beam related problems such as target location/alignment and magnetic horn failure, before they manifest themselves in the Far Detector data. The superstructure of the Near Detector weighs 980 tons and has 282 steel planes in the shape of a squashed octagon, shown in Fig. 4.6. While geometrically different from the Far Detector, the interaction region of the Near Detector, due to the low beam divergence, has the same magnetic field as that of the Far.

In the Near Detector, the scintillator modules have a staggered assembly, where every $5^{\text {th }}$ plane has scintillator coverage of $13.2 \mathrm{~m}^{2}$ (fully instrumented), while the 4 interleaved partially instrumented planes cover $6.0 \mathrm{~m}^{2}$. The partially instrumented planes provide sufficient coverage to identify the interaction vertex and measure hadronic shower activity of neutrino events, while the larger fully instrumented planes have an extended lever arm better suited for measuring the curvature of muons in the magnetic field. The Near Detector is separated for analysis purposes into two different sections: the "calorimeter" being the upstream 121 planes and the "spectrometer" being the downstream 161 planes.

The calorimeter section is used to identify the neutrino interaction vertex, provide containment for hadronic and electromagnetic showers and provide granularity for muon track reconstruction. In this region, every steel plane has an associated scintillator plane, making all 121 planes active. The 161 planes in the spectrometer are used to measure track curvature and are instrumented with fully instrumented planes in only every $5^{\text {th }}$ plane. The strips in the spectrometer are multiplexed so that 4 strips are read out by one channel in the electronics and then topographically separated in the offline reconstruction to identify energy deposits in the detector. The summing in the spectrometer is not present in the calorimeter, where each strip 


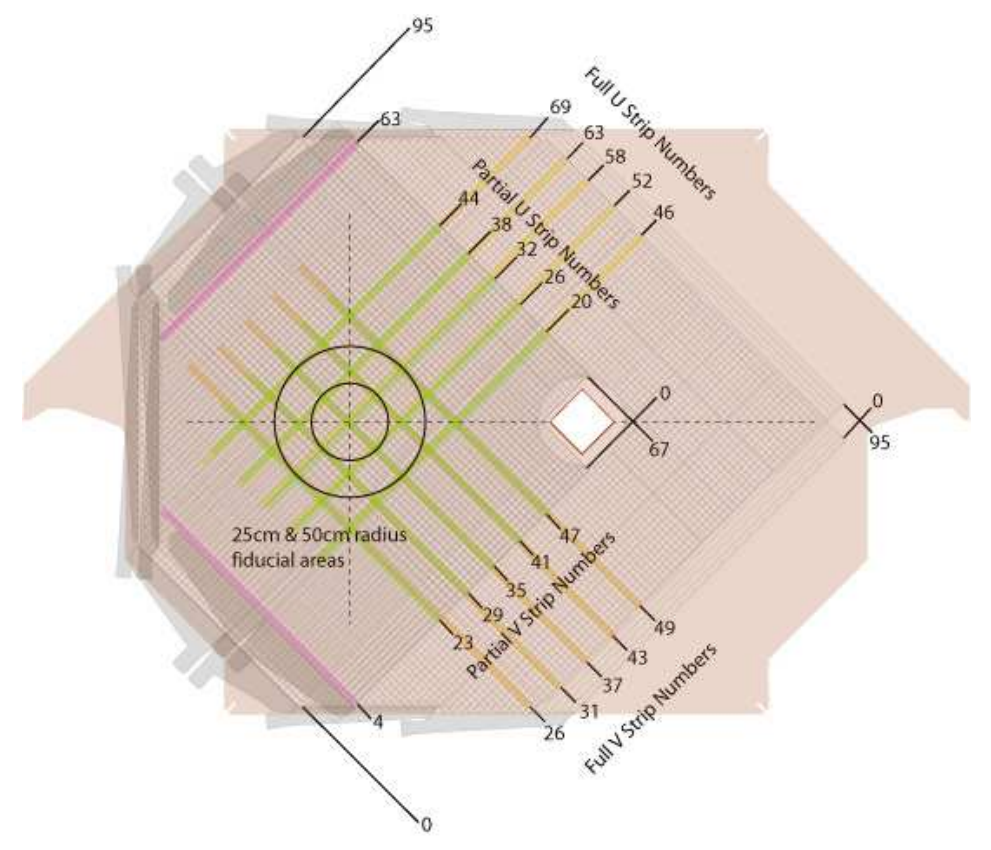

Figure 4.6: Diagram of the scintillator plane layouts for the Near Detector, where the NuMI beam spot is marked by the bullseye. This is a downstream view where the overlay of the partial and full planes illustrates the difference in coverage.

is read out independently with a dedicated electronics channel.

The Near Detector is read out on one side only. The scintillator module manifolds on the east side, opposite the electronics, are fitted with connectors that reflect the light back into the WLS fibers.

\subsubsection{Near Detector Electronics}

The high event rate in the Near Detector, from both cosmic rays and NuMI spills, requires electronics that operate without dead time. To convert the analog signal supplied by the Hamamatsu M64 PMT, MINOS uses the Fermilab developed Charge(Q) to Current(I) Encoder (QIE) circuit design. The QIE chip has 4 different phases, each taking 19ns, to complete the analog to digital conversion: integration, range select, digitization and reset. In the integration phase the charge is split in a binary fashion $\left(\frac{1}{2^{N}}: \frac{1}{2}, \frac{1}{4}, \frac{1}{8}, \frac{1}{16}, \ldots\right)$ across 8 capacitors, integrated, and then 
driven to a set of comparators. The chip has 8 separate ranges for the analog to digital conversion, where in the range select phase the comparators uniquely identify which of the 8 ranges will provide the greatest accuracy (Fig. 4.7). During the digitization phase the voltage on the capacitor of the selected range is provided to the Fast ADC (FADC) for conversion, which has 8 bit precision. The reset phase flushes the charge from the capacitors.

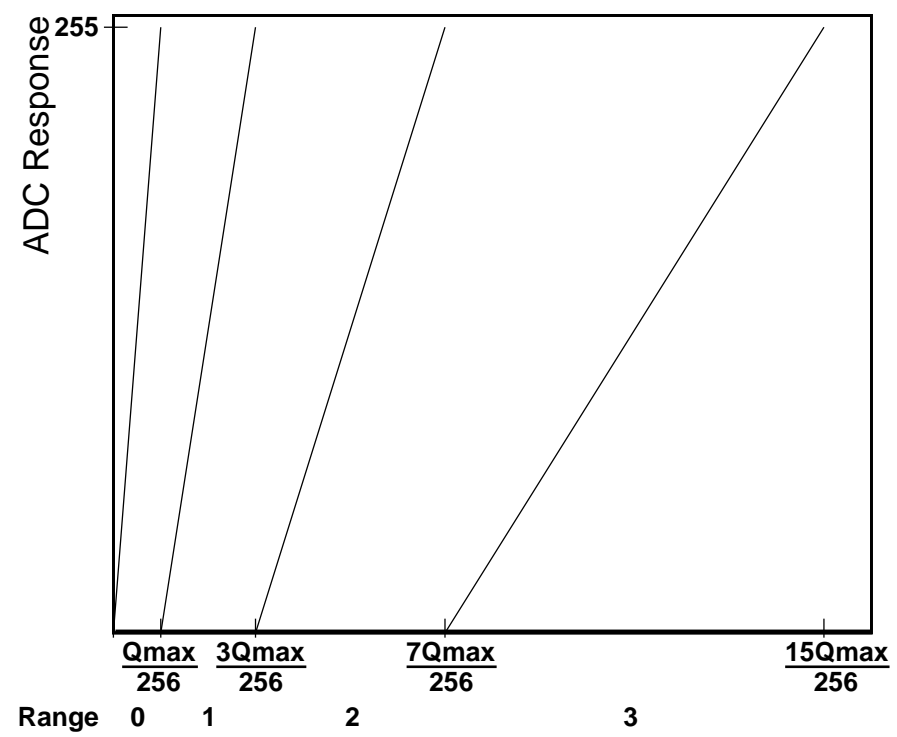

Figure 4.7: Ideal response of the different QIE chip ranges, where only ranges 0-3 are shown. The FADC digitizes to 8 bits, which dictates the precision of the conversion. The comparators select which of the appropriate ranges to use during the select phase of the QIE chip analog to digital conversion.

Data acquisition occurs only during the Integration phase, and in order to avoid $75 \%$ deadtime there are three extra sets of integrating capacitors and range selector comparators. The QIE chip cycles through each capacitor set, allowing for all the different phases without dead-time. The end product is a 13 bit data 'word' that consists of 2 capacitor ID bits (0-3), 3 Range bits (0-8) and 8 FADC bits. Each QIE chip is stored on a daughter board, known as the MENU, which contains a First In First Out (FIFO) storage device for queuing data.

The time stamping, power, control and interface of the QIE chips is handled by 
a Front End Mother Board, known as a MINDER. Each MINDER monitors and controls 16 QIE daughter boards, which are read out in the event of 3 trigger types: spill, cosmic and calibration. The spill trigger is received from the clock system, where the MINDER prompts the 16 QIE chips to store all the data during the spill time window (SGate) from the QIE chips into the FIFO storage. At the end of the NuMI spill gate, the MINDER queries each MENU for processing. The cosmic trigger is signaled by PMT dynode activity on the various PMTs and data are read over a 150ns window. There is also a calibration trigger used to measure baseline detector activity.

\subsection{Far Detector}

The main purpose of the Far Detector is to measure the oscillated $v_{\mu}$ spectrum from the NuMI beam. Additional oscillation analyses concerning $v_{e}$ appearance and existence of a sterile neutrino $v_{\mu} \rightarrow v_{s}$ also rely on using the Far Detector for measuring oscillated spectra. The large size, rock overburden and ability to distinguish between $v_{\mu}$ and $\bar{v}_{\mu}$ also makes the Far Detector an attractive tool for cosmic and atmospheric non-oscillation physics.

The detector is situated $735 \mathrm{~km}$ downstream of the NuMI target, and 2341 feet underground in the Soudan Underground Laboratory. The detector consists of 484 active planes of alternating steel and scintillator, separated into two supermodules and capped at each end by an extra steel plane. The planes, octagonal in shape and 8 meters from one side to the opposite, are unlike the Near Detector in that each

of the 484 planes has $53 \mathrm{~m}^{2}$ scintillator coverage. At the center of each plane is a coil hole, fitted with a collar upon installation, through which the magnet coils are routed to provide the magnetic field.

The detector is read out on both sides and multiplexed so that 8 strips, each separated by $1 \mathrm{~m}$, are optically summed on the same photomultiplier channel. The 


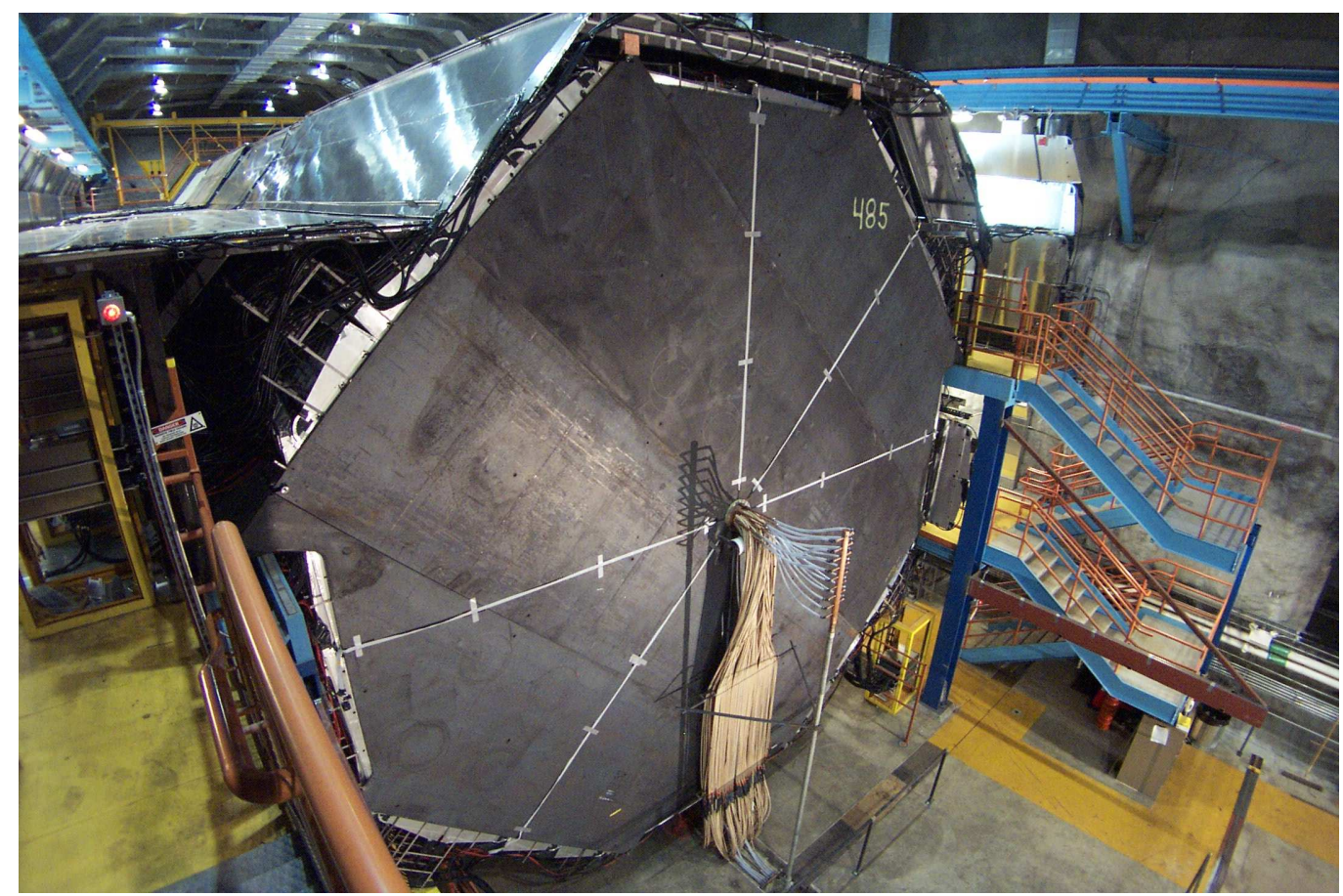

Figure 4.8: The end of supermodule 2 of the Far Detector. This view is looking south towards Fermilab from the observation deck. The coil cables running through the hole in the middle constitute the magnetic field coils, and the silver reflective cover on top of the detector are the scintillator modules of the Veto shield.

mapping occurs in multiplexing (MUX) boxes where fibers from the scintillator strips are routed between three different PMTs housed in the box (Fig. 4.9). The map differs between the two sides allowing for separation of strip hits [69].

The Far Detector is equipped with a veto shield, constructed to cover the major downward angles and locations of background cosmic rays. It is made of scintillator modules stacked two deep to provide adequate coverage and reliability of detecting cosmic rays.

\subsubsection{Far Detector Electronics}

The rate of neutrino and muon events at the Far Detector from both the NuMI beam and cosmic rays is significantly less than in the Near Detector. The electronics were designed for this low rate underground environment in that they have better resolution than the Near Detector electronics, but do suffer minimal dead-time, 


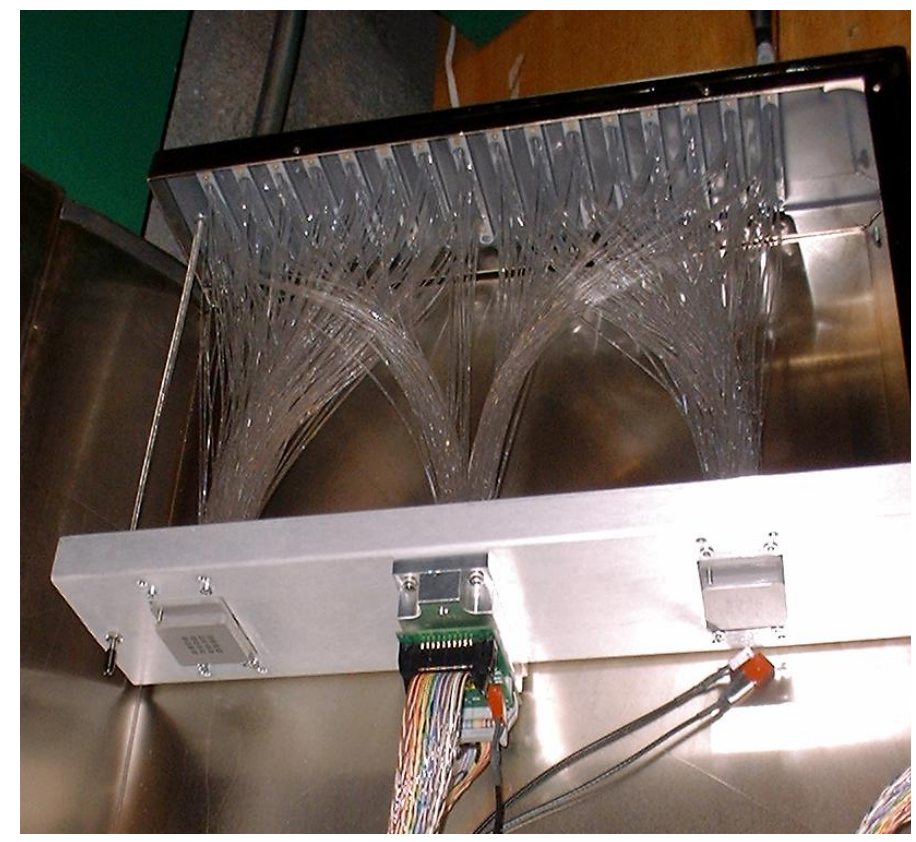

Figure 4.9: The clear fibers from the detector are shown coming in from the top of the MUX box. Multiplexing happens between strips more than 1m apart in the Near Detector grouped into bunches of eight fibers which illuminate one pixel of the PMT.

though this is negligible for an event rate of $0.5 \mathrm{~Hz}$ for cosmic ray muons[70] and 1-3 NuMI neutrino events per day[71].

Light from the Far Detector illuminates one of the 16 pixels on a given Hamamatsu M16 PMT. The readout of each PMT is handled by a VA chip, which processes data across 17 of its 32 channels, where the 15 remaining channels are unused. Each channel on the chip consists of a charge sensitive preamplifier, a shaper, a track and hold stage as well as an output switch. The VA chips are housed three to a group on a VA Front-end Board (VFB), which controls the power and initial time-stamping of the data. The analog signal from the VA chips is digitally converted by the VA Mezzanine Module (VMM), which connects to two VFBs. The VFBs operate in slave mode, controlled by the VA Read out Controller (VARC). The VARC initiates the triggering and also controls the electronics calibration. 


\subsection{Calibration Detector}

The Calibration Detector (CalDet) was, in essence, a $1 \mathrm{~m} \times 1 \mathrm{~m} \times 3.6 \mathrm{~m}$ section of the Near or Far Detector, with the exception that it was not magnetized. The CalDet weighed approximately $15 \mathrm{~T}$, with 60 planes of steel and scintillator. The main purpose of the detector was to achieve the relative calibration between the Near and Far Detector. Since the detector was the first to be constructed, it also served as a testbed for detector components and calibration procedures that would later be applied in the other two detectors.

\subsubsection{The CalDet setup}

To test the absolute and relative calibration of the Near and Far Detector, the CalDet was specifically constructed at CERN at the East Area Hall in the T11 and T7 test beams (Fig 4.10). The setup used the CERN Proton Synchotron (PS) to provide a proton beam incident on a user selected target to produce a secondary beam of pions, protons, electrons and muons. Similar to the NuMI beam, the particle beam goes through focusing and momentum selection to attain a particle beam that is energy specific. The use of the CalDet can also be extended to examine detector response characteristics to a momentum tuned beam of various particles such as pions, kaons, electrons and protons. 


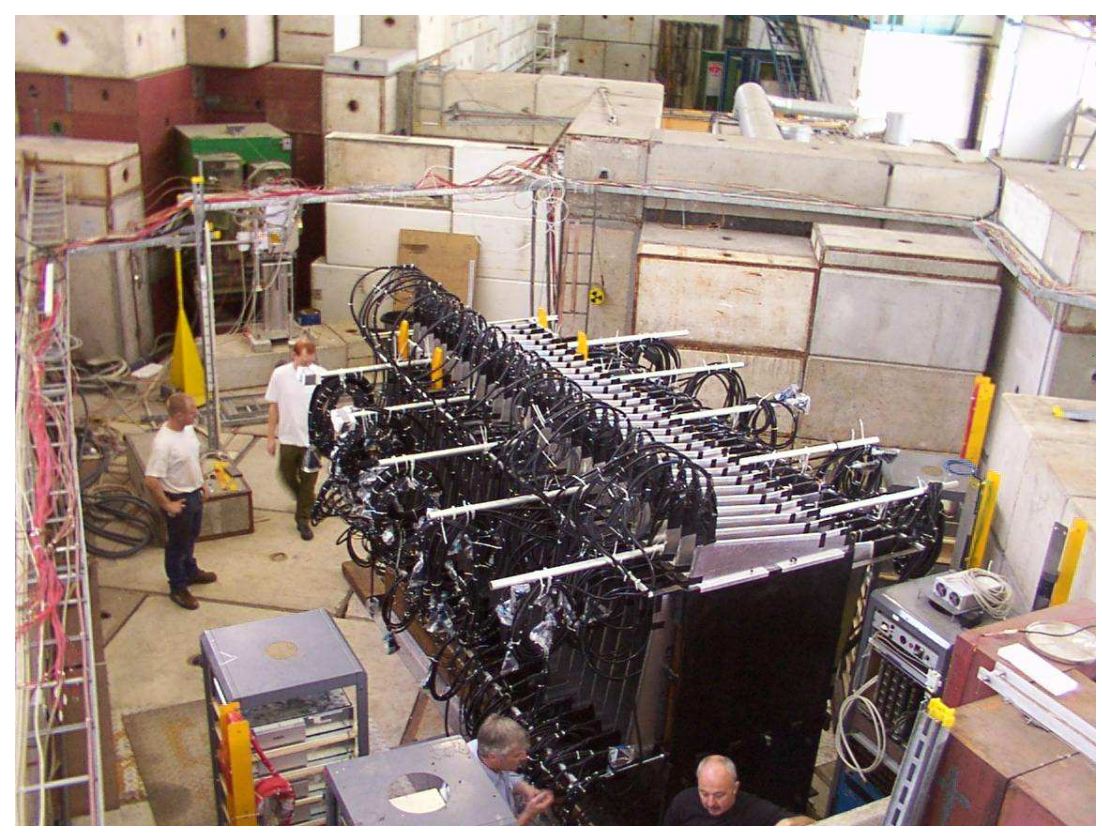

Figure 4.10: The Calibration Detector in the PS hall at CERN. The green box (top left of picture) is the electromagnet that is used to bend the beam path. 


\section{Chapter 5}

\section{Event Selection, Far/Near}

\section{Extrapolation and Systematic}

\section{Uncertainties}

The MINOS detectors are designed and optimized to detect charged current interactions from muon neutrinos generated by the NuMI beam. The detectors also serve to measure the rate of neutral-current events which manifest as a hadronic shower from the exchange of the $Z$ boson. Because the $Z$ couples only to the three light active flavors of the neutrino, a deficit of $\mathrm{NC}$ events over a long baseline would be indicative of a neutrino transition to a non-active or sterile flavor. Although there has been little indication of a sterile neutrino by previous experiments [72, 30, 73], MINOS is the first accelerator long-baseline neutrino experiment to look for a sterile neutrino, $v_{s}$.

The analysis first selects quality reconstructed neutrino events as described in Secs. 5.1 5.3 and then separates the sample into CC and NC groups as described in Sec. 5.4. Using the NC and CC selected events, energy spectra predictions are made in Sec. 5.5, which extrapolate the Near Detector data to the Far Detector. The nature, size and effect of the systematic uncertainties is included and compar- 
isons between the Far Detector data and predicted energy spectra are made using different neutrino oscillation models in Sec. 5.6. The analysis is conducted under 'blind' conditions, where the Far Detector data remains unexamined as a function of energy until all steps of the analysis are vetted. All procedures, cuts and energy ranges are determined using Monte Carlo studies, where Ch. 5 illustrates using modified Monte Carlo events as 'fake' data to test methods and algorithms.

\subsection{Event Reconstruction}

MINOS uses partially active segmented detectors, where the active segments (scintillator) are separated by inactive regions (air gaps and steel plates). The consequence of this design is that continuous particle propagation is recorded as discrete hits in scintillator strips. To make sense of the individual hits, an event reconstruction provides the energy, direction and charge sign of a particle interacting in the detector. The path of the reconstruction procedure is:

I. Record activity in the detector that satisfies criteria related to minimum and maximum detector activity.

II. Isolate, identify and assemble the activity in the detector into individual scintillator strip hits containing time, location and energy.

III. Topologically cluster the strip hits into groups relating to tracks and showers.

IV. Determine the direction and energy of tracks and showers, as well as the charge sign of tracks.

The assemblage of information from detector activity into particle properties happens in stages, where each stage produces 'candidate' objects. Each candidate object is the representation of a potential hit, strip, shower, track or event that can 


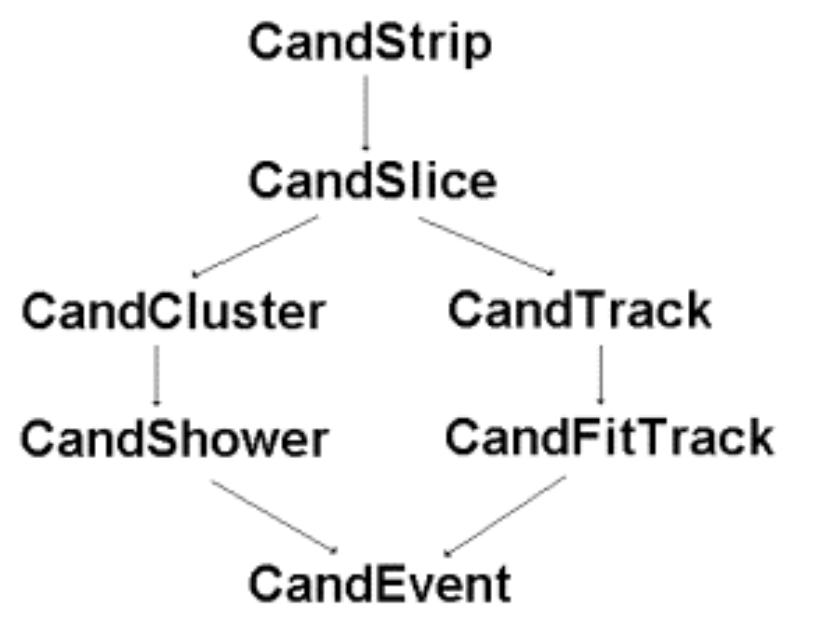

Figure 5.1: Flow diagram of the MINOS reconstruction. Each stage produces a candidate object that is broken down into the respective likelihood of being either a track or shower. Events are then constructed as an amalgamation of tracks and showers associated with a unique primary particle interacting in the detector.

be used in analyses. All events in MINOS undergo the same reconstruction and are later classified as being one of the various possible interaction types.

All the activity for a single (or group of) particle interaction(s) which are clustered in time are classified as 'snarls' in the MINOS reconstruction lexicon. On the opposite end of the spectrum the information relating to the location (plane and strip), time and energy corresponding to a given hit is termed a "CanDigit". The information in the CandDigit is stored before any calibrations have been applied and all the hits in the detector are added to a list that is used to create showers and tracks (Fig. 5.1). The reconstruction minutiae pertaining to muon tracks will not be addressed here because the focus of this dissertation rests with using $\mathrm{NC}$ events, which are identified by their hadronic shower.

\subsubsection{CandStrip}

A CandStrip object stores hits that are localized in time and occur on the same strip within the detector. Excluded from this list are PMT cross-talk hits, where hits are deemed cross-talk if the charge on the PMT pixel is less than $10 \%$ of the summed 
charged of the 4 surrounding PMT pixels in addition to the hit, or

$$
\text { cross-talk : } \frac{Q_{h i t}}{Q_{h i t}+\sum_{i}^{4} Q_{i}}<0.10
$$

where $Q_{h i t}$ is the charge on the PMT pixel for the hit strip and $Q_{i}$ the charge on each surrounding PMT pixel. The charge on the 4 nearby PMT pixels is only summed over a $40 \mathrm{~ns}$ time window from the start of the original hit. A value less than $10 \%$ is an indication that that the CandStrip in question is generated not by light on the strip but by electrons or light from a nearby pixel on the PMT base, which should be discounted.

Besides satisfying the cross-talk criteria, the hits forming a CandStrip are localized in time and must be separated by $<60 \mathrm{~ns}$ from adjacent hits and $<120 \mathrm{~ns}$ from the initial hit of the CandStrip. These requirements ensure that there are no significant time gaps between hits on a strip and that there is a sensible duration of activity for a CandStrip object. Hits that violate the time requirements become the initial entry in a new CandStrip object.

\subsubsection{CandSlice}

The next step of the reconstruction is to identify single physics events, CandSlices, from the array of CandStrips. In the Far Detector, the event rate is sufficiently low that a snarl often contains only one physics event. The high event rate in the Near Detector (7-12 events per NuMI spill for $2.5 \times 10^{13} \mathrm{POT} /$ pulse) means that one snarl has multiple events and requires sorting in order to identify the individual physics events. 


\subsubsection{CandCluster}

A CandCluster is a 2D assortment of grouped strips in the same U-view or Vview 1 slice and is the first step towards defining showers. The algorithm is viral in behavior, in that a CandCluster starts out as a single strip and adds to itself all strips that satisfy specific time/space criteria. The recently added strips in the cluster then use the same criteria to accumulate more nearby strips. The process continues until reaching the limit, where all remaining strips not in a cluster exist beyond 'infection'. The procedure repeats itself until all strips are absorbed into a CandCluster. Finally the CandClusters are designated as being either showerlike or track-like depending on the properties of their primary strip ${ }^{2}$. Shower-like CandClusters have a primary strip that has neighbors on the same plane, while track-like clusters have a primary strip that is isolated and has no nearby neighbors on the same plane.

\subsubsection{CandShower}

A CandShower is a 3 dimensional object created from CandClusters. Each 2D cluster is matched to its $\mathrm{U}$ or $\mathrm{V}$ view counterpart, making sure that they are both near in time and size. For two clusters disparate in size, the smaller of the clusters must span at least $50 \%$ of the planes of the larger in order to be matched into a CandShower.

The vertex and direction of the shower are important in determining whether the neutrino event is generated by the NuMI beam and that the event has adequate shower energy containment, i.e. exists completely within the fiducial volume. The vertex is assigned as the $\mathrm{U} / \mathrm{V}$ charge weighted position of the 5 most upstream

\footnotetext{
1 The strips in each plane are oriented at a 90 degree rotation to the preceding plane. When overlayed, a U-view and a V-view strip make an "X"; one going top left to bottom right, while the other is oriented bottom left to top right.

${ }^{2}$ Ultimately each Working Group defines their own criteria and procedure to identify specific event interaction types that optimize their physics analyses.
} 
planes of the shower. The direction is established from a linear fit to the charge weighted transverse position in each plane versus the longitudinal position. The weighted linear fit in each view provides the slopes $\mathrm{du} / \mathrm{dz}$ and $\mathrm{dv} / \mathrm{dz}$, which are then used to obtain the direction cosines of the shower axis.

\subsubsection{CandEvent}

After using fitting methods to produce tracks and shower, a CandEvent object is created, which associates tracks and showers with the primary physics event. A CandEvent may contain more than one shower or track and allows sharing of hits, or CandDigits, between them. The vertex, or primary neutrino interaction point, is defined in the NC Analysis as being the vertex of the shower, unless the primary track is longer in the horizontal direction than the shower.

\subsection{Near Detector Event Selection}

The events assembled by the MINOS reconstruction are the result of activity throughout the whole detector. Included in the events is a subset generated from non-NuMI sources: cosmic rays and noise. In order to ensure that the events used in the Neutral-current analysis are well defined neutrinos from the NuMI beam a series of cuts are used to identify good neutrino events.

In order to establish well-defined neutrino events, 'good' events are required to have a reconstructed vertex that is $>50 \mathrm{~cm}$ from the edges of the partially instrumented planes, including the center of the magnetic coil hole, as in Fig. 5.2. The fiducial volume vertex requirement eliminates events that may have been generated outside the detector, while also providing containment of the shower dispersion from a NC interaction. Shower containment is necessary in order to provide the most accurate energy reconstruction of shower inducing events. Besides the detec- 


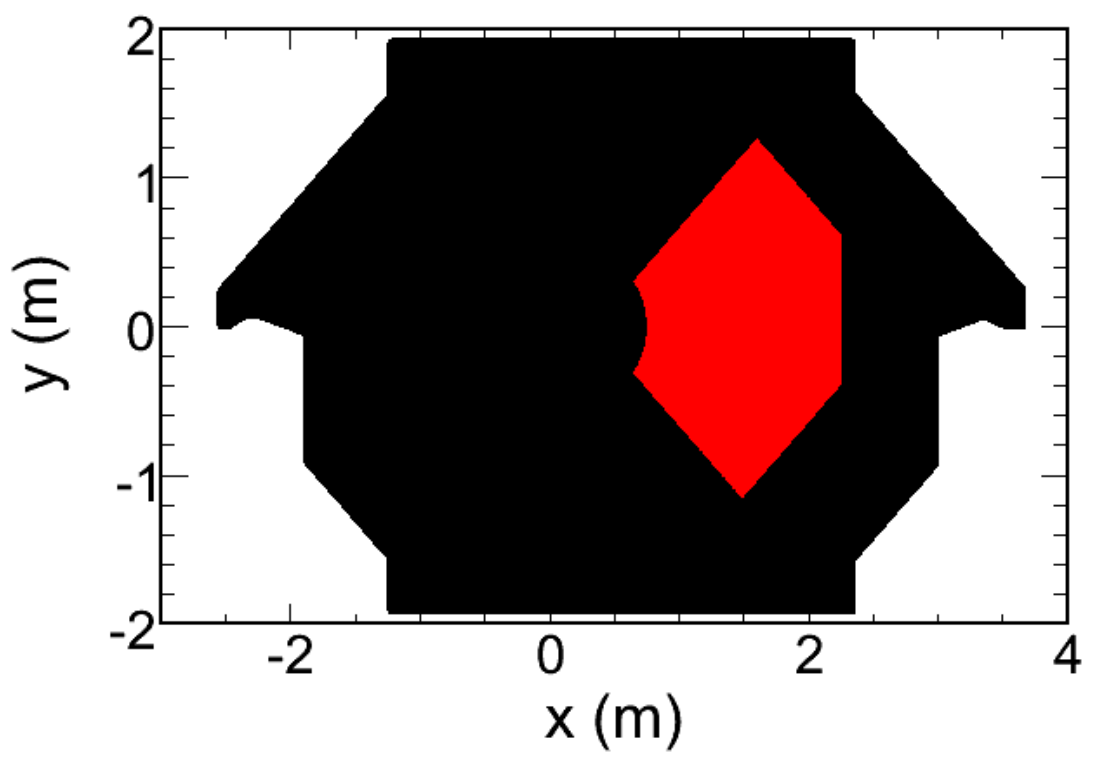

Figure 5.2: Fiducial volume diagram of the Near Detector. The light red signifies the fiducial volume, while the black represents the outline of the steel planes used in the Near Detector. The magnetic coil is located on the left side of the detector face, situated at $(0,0)$ in the diagram.

tor edge cut, an additional longitudinal vertex cut $1.7 \mathrm{~m}<z<4.7 \mathrm{~m}$, or 30 planes $<z<80$ planes, is applied to also provide shower containment.

The high rate of neutrino events in the Near Detector requires an extra set of pre-selection cuts to mitigate the effect of the following reconstruction pathologies: split events, vertexing failures and 'others'. Split events are caused when a single neutrino event is split into multiple events during reconstruction; vertexing failure is when an event outside the fiducial volume is reconstructed to lie within the fiducial volume; the majority of the catch-all set of 'other' pathologies occur for low numbers of reconstructed strips, such as large gaps within showers as well as sparse showers. Requiring that showers contain $>4$ strips minimizes events with poor coverage and significantly reduces the 'other' class of pathologies. The totality of the cuts to remove poorly reconstructed events, detailed in the upcoming Secs. 5.2.1 and 5.2.2, result in a substantial decrease in the number of poorly reconstructed events and, as an additional benefit, decrease the charged-current 


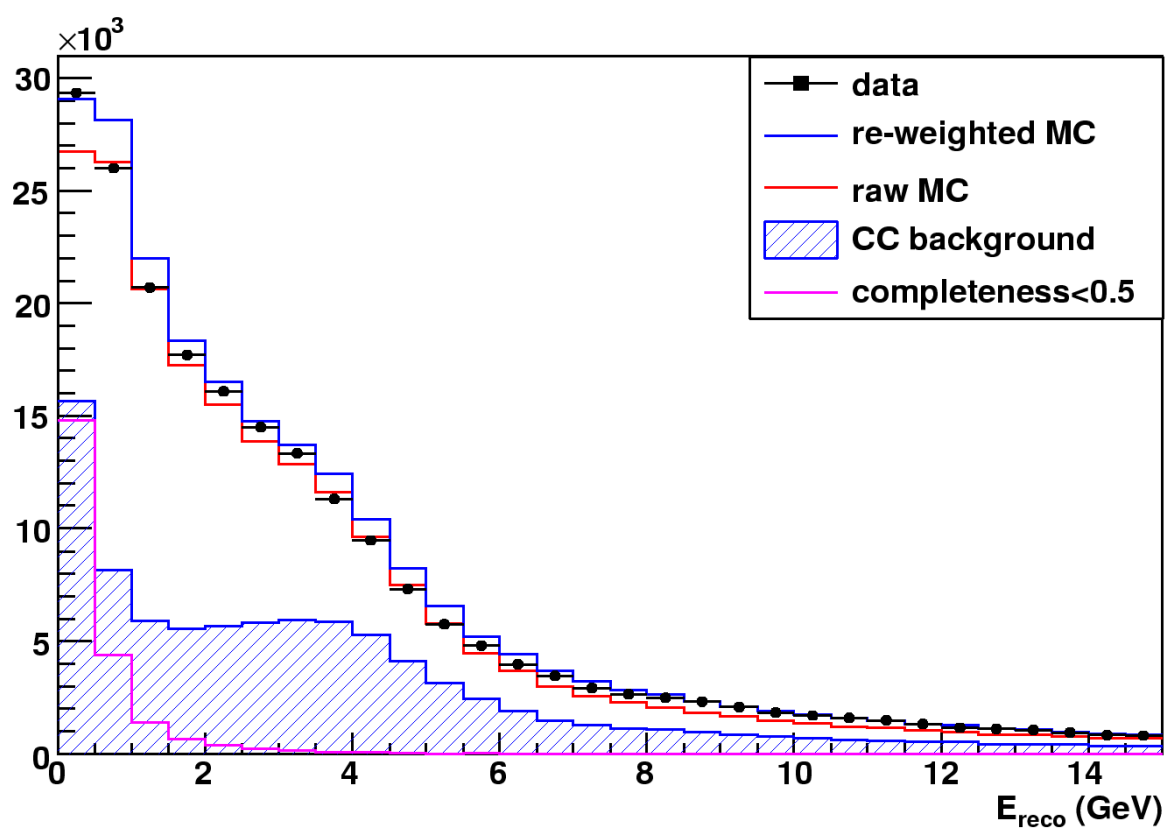

Figure 5.3: Plot showing the NC energy spectrum the Near Detector before application of the cleaning cuts to remove reconstruction pathologies. The hatched blue represents the misidentified CC background while the magenta is the low completeness events. The first two energy bins, $<1 \mathrm{GeV}$, show a significant contribution from both "low-completeness" events as well as charged-current background. The blue and the red lines show the Monte Carlo with and without the SKZPTV beam re-weighting.

background (Figs. 5.3 and 5.4).

\subsubsection{Split Events Pathology}

Split events result in extra reconstructed events at low energy in the Near Detector. Not only does the pathology create a sizable background that alters the neutrino energy spectrum and double counts neutrino interactions, but lower energy events are more likely to be classified as neutral-current events, regardless of their true interaction type.

The size of the pathology is evaluated in the Monte Carlo event set based on "completeness", $c$, of the reconstruction, where $c$ is defined as the ratio of the reconstructed energy of an interaction to the total visible energy of the interaction. 


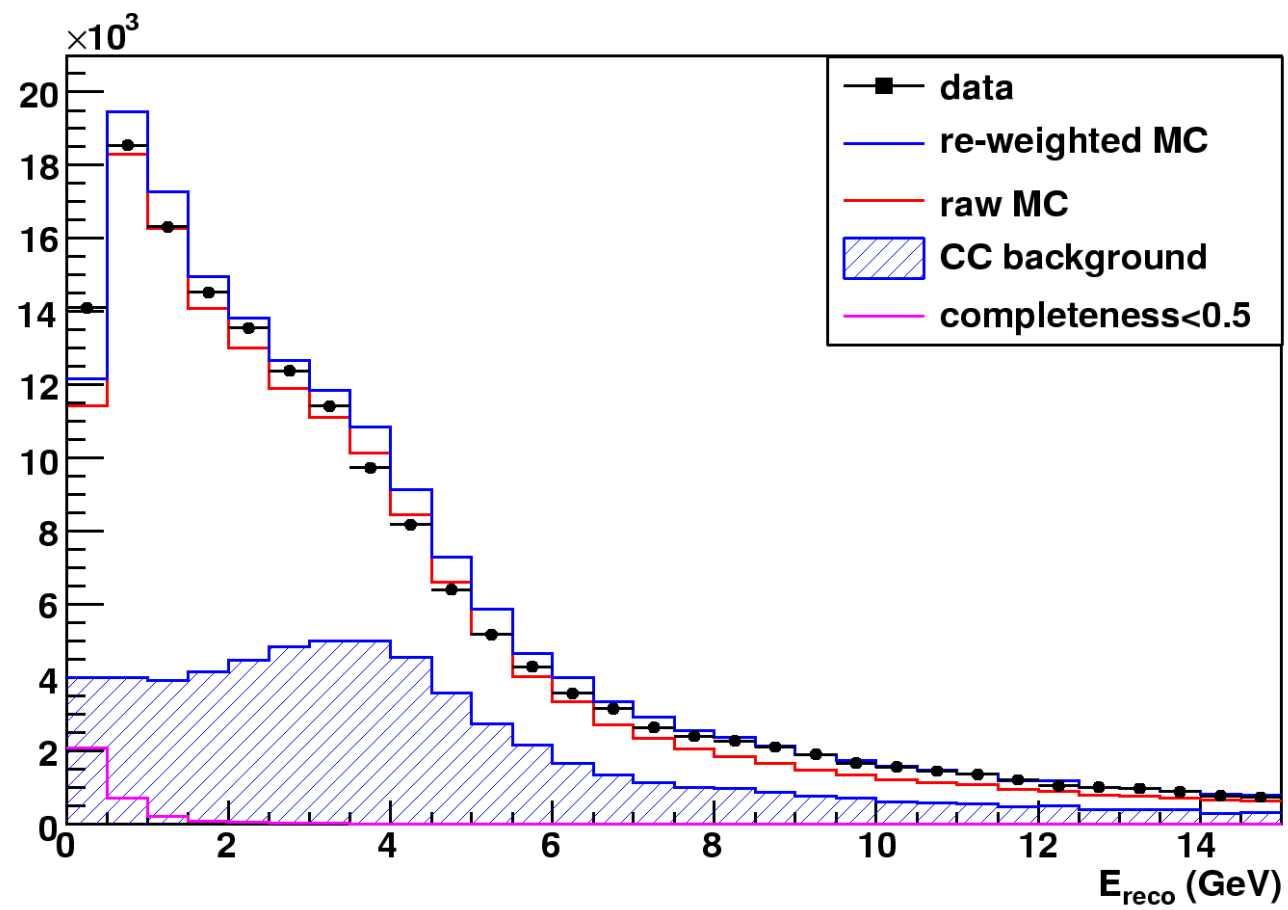

Figure 5.4: Plot showing the NC energy spectrum in the Near Detector after the cleaning cuts are applied. The amount of low completeness events $<1 \mathrm{GeV}$ is dramatically reduced upon application of the cleaning cuts and all but wiped out $>1 \mathrm{GeV}$. The cleaning cuts also produce a reduction in the mis-identified CC background in the region $<2 \mathrm{GeV}$. Further cuts remove background and increase data-MC agreement. 


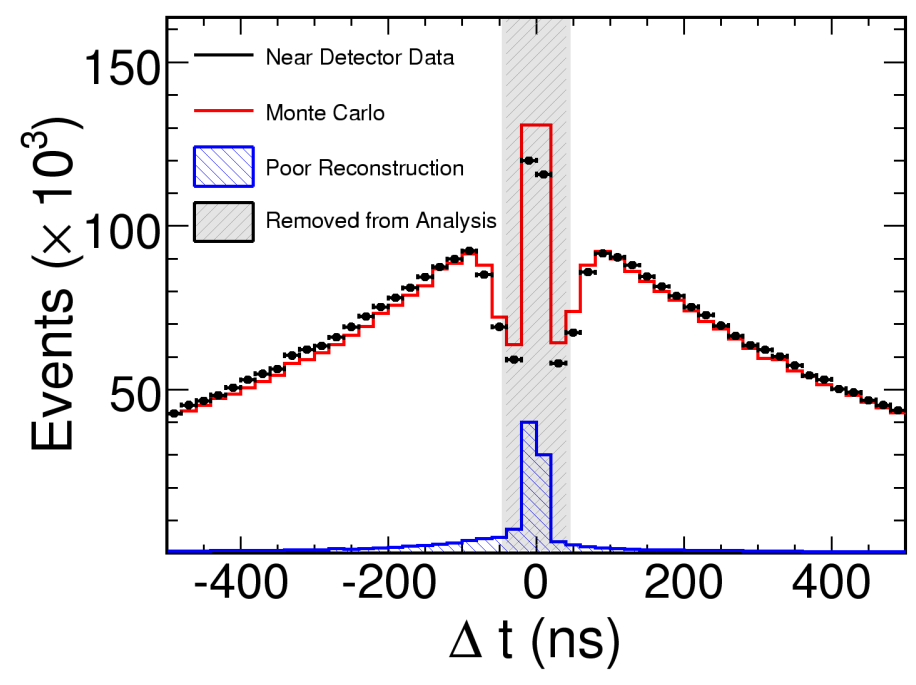

Figure 5.5: Shows the difference in time between reconstructed events. The black points represents the data, red the Monte Carlo and the hatched blue area denotes low completeness events, $c<50 \%$. A large fraction of the low completeness events have $|\Delta t|<40 \mathrm{~ns}$, which corresponds to the Time separation between events cut represented as the grey hatched region. All events in this region are removed from the analysis.

A small fraction, $\sim 4 \%$, of the total events passing the fiducial volume cuts have $c<50 \%$, as shown in Fig. 5.3. In the situation where an event is split into multiple events, each separate event is reconstructed near the other(s) in both time and space. Based upon the low strip count and localization topologies, events are kept if they satisfy the following

- Minimum size - $N_{\text {strips }}>4$

- Time separation between events - $|\Delta t|>40 \mathrm{ns,}$ which is shown in Fig. 5.5

- Spatial separation between events - $|\Delta z|>1 \mathrm{~m}$ if the vertex occurs within $40 \mathrm{~ns}<|\Delta t|<120 \mathrm{~ns}$ of another event

The cuts provide a dramatic reduction in the amount of low completeness events, see Fig. 5.4 


\subsubsection{Vertexing Failure Pathology}

Events that suffer the vertexing failure pathology are predominantly the result of steep, or very vertical oriented, showers producing many strip hits across a sparse number of planes or non-fiducial particles which enter through the uninstrumented portion of the detector. A steepness variable, $s$, is used to characterize very steep showers, where the definition is

$$
\text { steepness }=\frac{\text { event strips per plane }}{\# \text { planes }}
$$

Events are removed from the analysis if $s>1.0$.

A non-fiducial particle which either scatters or decays to create a steep shower within the fiducial volume will often be preceded by activity at the edges of the detector. The sparsely instrumented detector regions outside the fiducial volume can be used to identify edge activity and act as a veto shield for events with true vertexes outside the fiducial region. The veto region is defined in the U-view as > $2.27 \mathrm{~m}$ or $<-0.24 \mathrm{~m}$ and in the $\mathrm{V}$-view $<-2.27 \mathrm{~m}$ or $>0.24 \mathrm{~m}$. Events are classified as having a vertexing failure and removed if they satisfy all of the following conditions:

- Event energy $<5 \mathrm{GeV}$

- Shower length is greater than track length

- Summed energy of strips in veto region, all being $<40 \mathrm{~ns}$ from the start time of the event, exceeds noise threshold

All veto region hits are considered in the calorimeter and all strips are considered, not just those satisfying reconstruction requirements. Inclusion of every hit makes the veto variables a low level quantity and robust to potential pathologies stemming from the slicing or shower reconstruction. 


\subsection{Far Detector Event Selection}

Similar to the Near Detector, establishing a fiducial volume is the first order of business for event selection in the Far Detector. The fiducial region is defined as $>50 \mathrm{~cm}$ from the outside edge of the detector and $>45 \mathrm{~cm}$ from the center of the detector/coil hole. The first 4 planes, or initial $23.28 \mathrm{~cm}$, of the two super-modules are used to veto events and the event vertex must be $>100 \mathrm{~cm}$ away from the end of each super-module in order to provide shower containment. The vertex of each event is the same as the Near Detector, where the shower vertex is used except when the primary track in the event is longer than the shower.

The selection of events in the Far Detector is subject to different pathologies than those present in the Near Detector. The main sources of background from non-neutrino sources in the Far Detector are from light injection, electronics or fiber noise, and cosmic ray muons. Further, backgrounds that are associated with neutrinos are the result of reconstruction failures or events with a timestamp out of the NuMI beam spill window.

\subsubsection{Non-neutrino Pathologies}

The Light Injection (LI) system, described in Sec. 4.2.1, is a calibration tool that measures PMT response to light from an LED pulsing numerous times at linearly increasing pulse heights. Because one of the goals is to measure short-term drifts, the LI system frequently pulses in coincidence with NuMI neutrino spills, creating light in the detector that can erroneously be reconstructed as neutrino events. A set of cuts, together referred to as LISieve, are used to identify Far Detector events generated by the LI system[74]. All the following cuts must be satisfied in order to remove an event: 
- Hits per plane - During LI pulsing, 8 or 10 strips per plane are directly illuminated. Even though only 8 or 10 are pulsed, cross-talk on the PMT can cause non-illuminated strips to record activity. Events that have a mean of more than 3 strips hit per plane are indicative of LI pulsing.

- East-West asymmetry - LI Pulsing occurs on a single side of a fiber during calibration, creating an asymmetry of the light levels between the East and West side. A neutrino event that does not create hits in the exact center of the detector also produces an East-West asymmetry but less than the LI which pulses at the absolute edge of the scintillator planes. To gauge the level of the asymmetry the following discriminator is used,

$$
\mathrm{A}_{W E}=\left|\frac{\sum \mathrm{PH}_{\text {West }}-\sum \mathrm{PH}_{\text {East }}}{\sum \mathrm{PH}_{\text {West }}+\sum \mathrm{PH}_{\text {East }}}\right|
$$

where $\mathrm{PH}_{\text {West }}$ refers to the pulse height at the West side of the WLS fibers. LI pulsing on the West side fiber ends will cause a larger value of $\mathrm{PH}_{W e s t}$ than $\mathrm{PH}_{\text {East }}$ and drive the value of $\mathrm{A}_{W E}$ toward one. Real physics events do not occur at the exact edge of the detector and are likely to have similar pulse heights on both the East and West side, which pushes the value of $\mathrm{A}_{W E}$ towards zero. The power of the variable to suggest LI activity is hampered when the pulsed light reaches a level that saturates the electronics. While the electronics nearest the pulsed strip end maintain maximum and constant readout over increasing light levels the opposite strip end electronics continue to approach saturation, which artificially decreases the asymmetry variable. Ultimately, events with either a saturated light level or an asymmetry value higher than 0.5 are likely caused by LI pulsing.

- Highest pulser box fraction - During LI activation, pulser boxes illuminate contiguous planes of the Far Detector, with each pulser box covering a differ- 
ent region. When an event contains hits across more than $85 \%$ of the planes in the pulser box region the hits may be caused by LI pulsing.

- Pulser box ratio - High energy physics events, specifically muons, produce numerous hits, which potentially span all planes across a pulser box region. Using solely the highest pulser box fraction criterion, long events would be considered indicative of LI pulsing. Because the LI executes in sequence there is only one pulser box flashing at a time. An event caused by LI should therefore have low activity in every other pulser box region of the detector compared to the one being pulsed. Thus, a ratio of the highest pulser box fraction to the second highest pulser box fraction below 0.05 is indicative of LI pulsing.

For all intents and purposes the LISieve cuts remove every event generated by LI, while also preserving all events caused by neutrinos. In a Monte Carlo data set with an exposure of $500 \times 10^{22}$ POT, which constitute $>15$ years of optimum MINOS data taking, the LISieve removed no real neutrino events. The rejection of LI events is $1: 10^{7}$ snarls, which equates to $>1000$ years of optimum MINOS data taking before a LISieve removes a true neutrino event.

The LI system is not the only non-neutrino cause of light within the detectors. Spurious low light in the Far Detector, known as singles noise, raises a problem not in background per se, but in creating dead-time in the electronics when digitizing hits of a non-neutrino nature. Singles noise is present in the Near Detector but does not cause any dead-time because it has continuous readout.

"Singles noise" is the result of electronics, dark noise from the PMTs, or spontaneous photon emission from the WLS fibers and scintillator[75]. Removal of sub-photoelectron(pe) noise from PMTs and electronics is accomplished with a $1 / 3$ pe threshold on the dynode trigger of the PMTs. The noise generated above the $1 / 3$ pe cutoff is very low for the M16 PMTs used in the Far Detector, leaving the 
scintillator and WLS fiber as the largest source of singles noise.

The major cause of the spontaneous scintillator/fiber emission is a confluence of intrinsic radioactivity in the materials as well as stress-induced phosphorescence from the relaxing of the WLS fibers[64]. The fiber noise is removed by a trigger that allows processing of the hits if 2 planes within 36 are above the $1 / 3$ pe threshold in coincidence.

The uncertainty inherent in the hadronic shower modeling as well as reconstruction pathologies lead to a 'Main Event' cut used when a Far Detector snarl contains two reconstructed events. With 1-3 neutrino events a day from $\sim 36,000$ individual NuMI spills, the likelihood of having two events in the same snarl is effectively nil, and signifies either a reconstruction failure or that at least one of the events is generated from a non-neutrino source. Outright exclusion of any snarl containing two events may introduce Monte Carlo and data discrepancies, especially as this pathology appears in unusual events that are difficult to model. A snarl with two events is kept for analysis if one of the events dominates the snarl, where the largest event must contain at least $75 \%$ of the energy in the event.

\subsubsection{Particle Pathology}

During a NuMI spill, all the hits in the Far Detector are digitized and recorded during a continuous time window of $\sim 100 \mu \mathrm{s}$, starting at $40 \mu$ s prior to the predicted beam arrival. With a cosmic muon rate in the Far Detector of $\sim 0.5 \mathrm{~Hz}$, the overlap between a cosmic muon and the spill window is minimal and the beam data will contain a cosmic event in every 20,000 snarls. While the likelihood is low that a cosmic and NuMI neutrino event will coexist in a snarl, topological cuts are applied to ensure that cosmic events are excluded.

The large rock overburden prevents all but the highest energy low angle muons from reaching the detector. Thus, the main feature of a cosmic ray in the Far 
Detector is that it is composed of a muon incident at a large angle to the z-axis. For cosmic rays that do reach the detector, the vertex location will often fall outside the fiducial volume and be excluded from the analysis. The remaining cosmic events are typified by their vertical path and can be largely removed by a cut on track angle. Assigned at the vertex, the track direction cosine is defined as $\left|p_{z} / E\right|$ of the muon, and all events with $\left|p_{z} / E\right|<0.4$ are classified as cosmic rays.

The aforementioned methods of identifying cosmic rays work well when the muon has a reconstructed track. However, the MINOS reconstruction algorithms are optimized to find tracks from the NuMI beam that are predominantly horizontal and often overlook high angle tracks. Failure to form a track causes the reconstruction to classify steep cosmic rays as a shower. The reconstruction failure of high angle tracks also complicates assigning the event vertex, and can introduce a background of non-fiducial volume events. While the reconstruction may mislabel an event and its vertex, the vertical nature is still evident and is a means to identify and remove the event.

A cosmic ray that is mis-reconstructed as a shower will contain a high number of strips hit in relation to planes spanned. To gauge the width to length relationship a transverse root mean sqaured (RMS) variable is created using the position of the strips. The RMS of strip position in meters is calculated separately in the $\mathrm{U}$ and $\mathrm{V}$ view and then added in quadrature to get the transverse RMS. Based on Monte Carlo studies, the following cut is made on the relationship between width, using the RMS of the strips, and length, using the planes spanned, to identify misreconstructed cosmic rays [74]:

transverse $R M S<3 \mathrm{~m}+0.1901 \log _{10}$ (planes in the shower).

The dispersion of neutrino induced showers scales linearly as the logarithm of the number of planes, hence the $\log _{10}$ term in eq.(5.4). For very high angle cosmic 


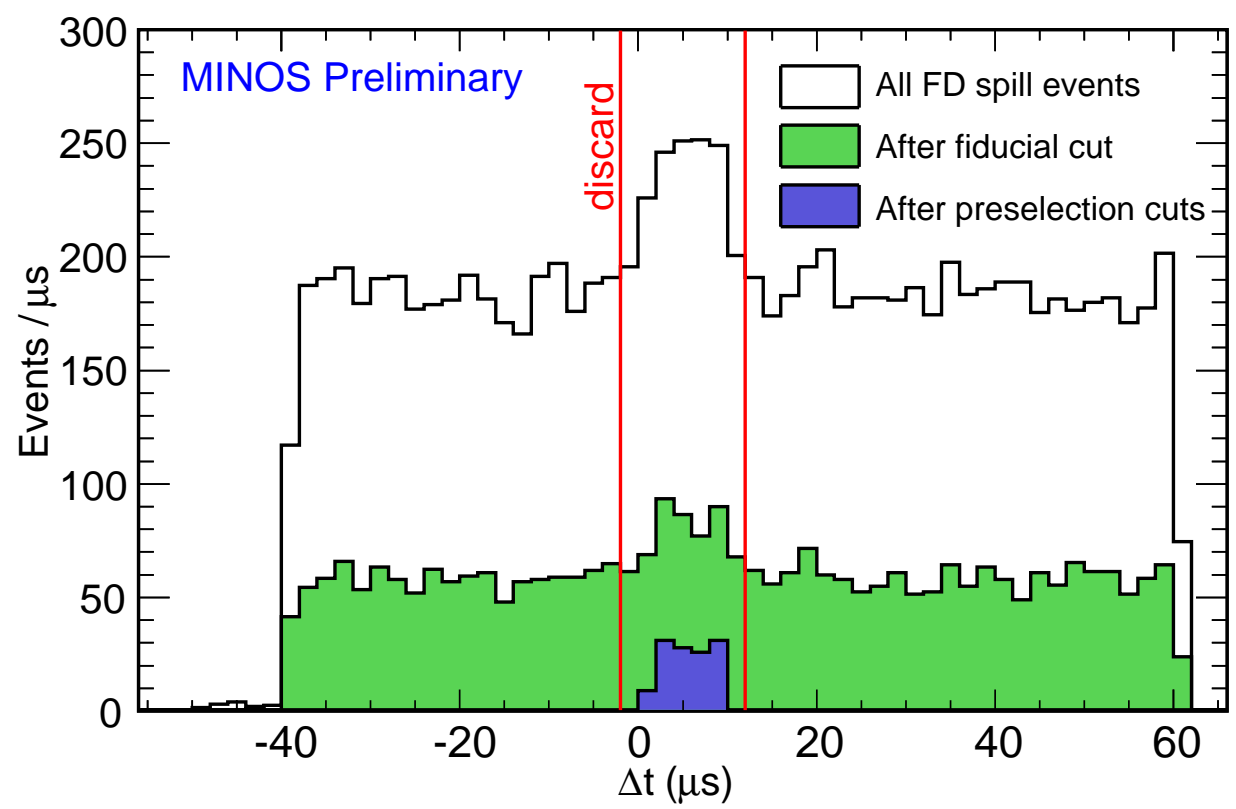

Figure 5.6: Number of neutrino events in the FD after timing, fiducial and preselection cuts. The preselection cuts largely exclude events of a non-NuMI origin. Even though the spill gate to accept events is $100 \mu s$, the quality of the timing shows that selected events lie between 0 and $10 \mu$ s; the predicted arrival time and duration of a NuMI spill.

rays that only hit a small number of planes an alternative width to length variable is used that identifies cosmic rays as

$$
\frac{\text { average strips per plane }}{\# \text { planes }}<1.0
$$

In total, events are kept if they satisfy the following cosmic ray removing criteria:

- Events with tracks whose z direction cosine $\left|p_{z} / E\right|>0.4$. Ensuring that the event has a satisfactory horizontal angle.

- Events whose showers have transverse RMS values that satisfy the condition 'RMS $<3+0.1901 \log _{10}$ (planes in the shower)'.

- The average number of strips per plane in the event divided by the number 
of planes in the event must be $<1.0$.

One of the most effective methods to ensure neutrino events are from a NuMI beam spill is to make selections based on the beam arrival time and duration of NuMI spill. The timing is established at each detector by a Global Positioning System (GPS) receiver which provides agreement between predicted arrival time and actual arrival time at the microsecond level. The duration of the beam spill is $10 \mu \mathrm{s}$, so the timing cuts are made at $-2 \mu \mathrm{s}$ and $+12 \mu$ s relative to the arrival of the beam. The timing cut coupled with fiducial and preselection cuts is shown in Fig. 5.6.

\subsection{Neutral-current/Charged-current Separation}

With good neutrino events in hand, as detailed in Secs. 5.2 and 5.3 , the neutralcurrent events are separated from the charged-current events using a cut based particle identifaction (PID) parameter[76]. The cut-based method was chosen over other separation methods, such as the k-nearest neighbour $(\mathrm{kNN})[77]$ and multiple artifical neural networks (ANN)[78, 79]. All methods provided similar purity as well as efficiency and the cuts based PID was chosen based upon its minimal susceptibility to systematic uncertainties versus the other procedures[80]. To illustrate the difficulty in separating events, Fig. 5.7 shows three distinct neutrino events from Monte Carlo, where distinguishing between a $\mathrm{NC}$ and a $\mathrm{v}_{e} \mathrm{CC}$ event is not trivial.

The separation procedure is as follows:

I. All events crossing $>60$ planes in the detector are classified as CC-like. Muons have a lower energy deposition than the products of an NC induced hadronic shower and therefore produce longer events. 
Transverse vs Z view - U Planes

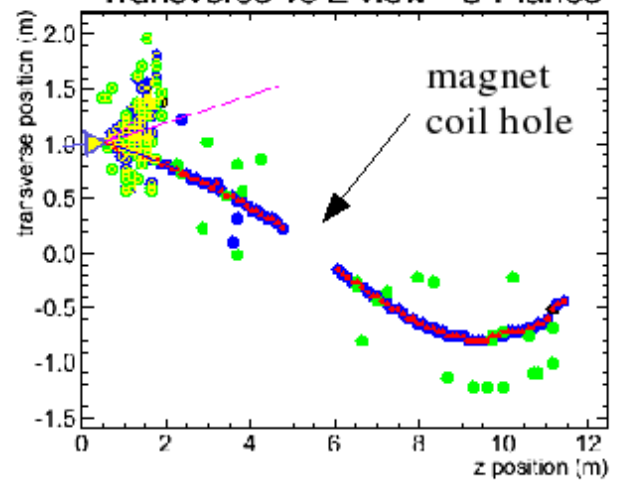

Transverse vs Z view - U Planes

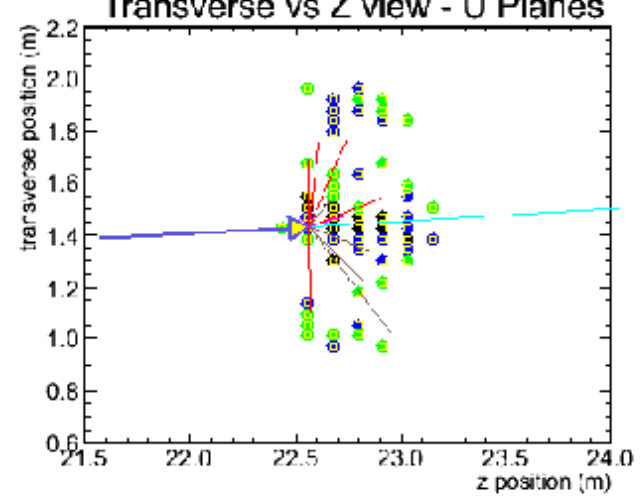

Transverse vs Z view - U Planes

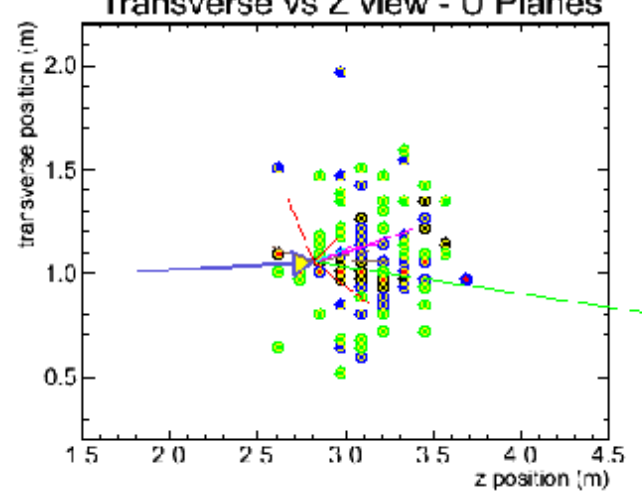

Transverse vs Z view - V Planes

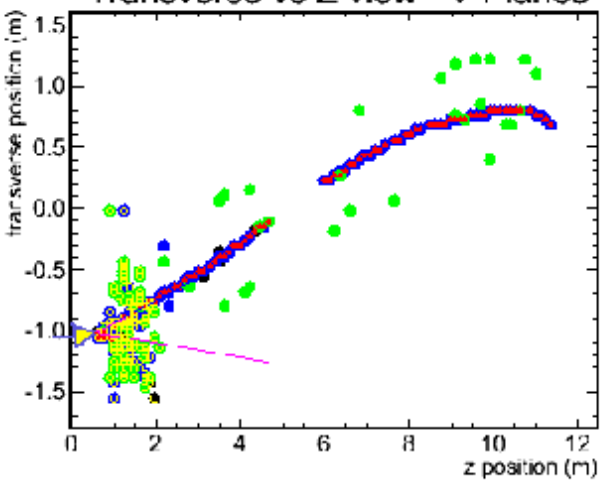

Transverse vs $Z$ view - $V$ Planes
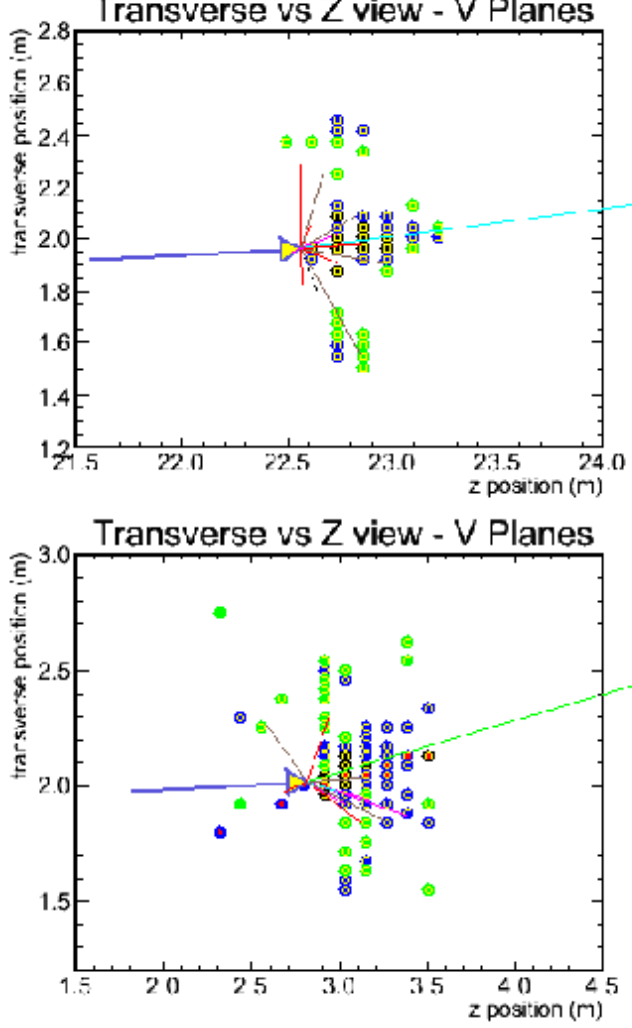

Figure 5.7: Event display in both the $\mathrm{U}$ and $\mathrm{V}$ view of 3 Monte Carlo events. The top plots are from a $22.2 \mathrm{GeV} v_{\mu} \mathrm{CC}$ event. Classic features such as a shower near the vertex and curvature in the presence of the magnetic field are clearly visible. Illustrating the effect of the magnet is the muon changing curvature after passage through the magnetic coil. The middle set of plots show a $11.6 \mathrm{GeV} v_{\mu} \mathrm{NC}$ event, which creates a short stubby shower. Similar to the $v_{\mu} \mathrm{NC}$ event the bottom displays show a $v_{e} \mathrm{CC}$ event. The topographical similarity will cause $v_{e}$ events to be a background of issue in later sections. 

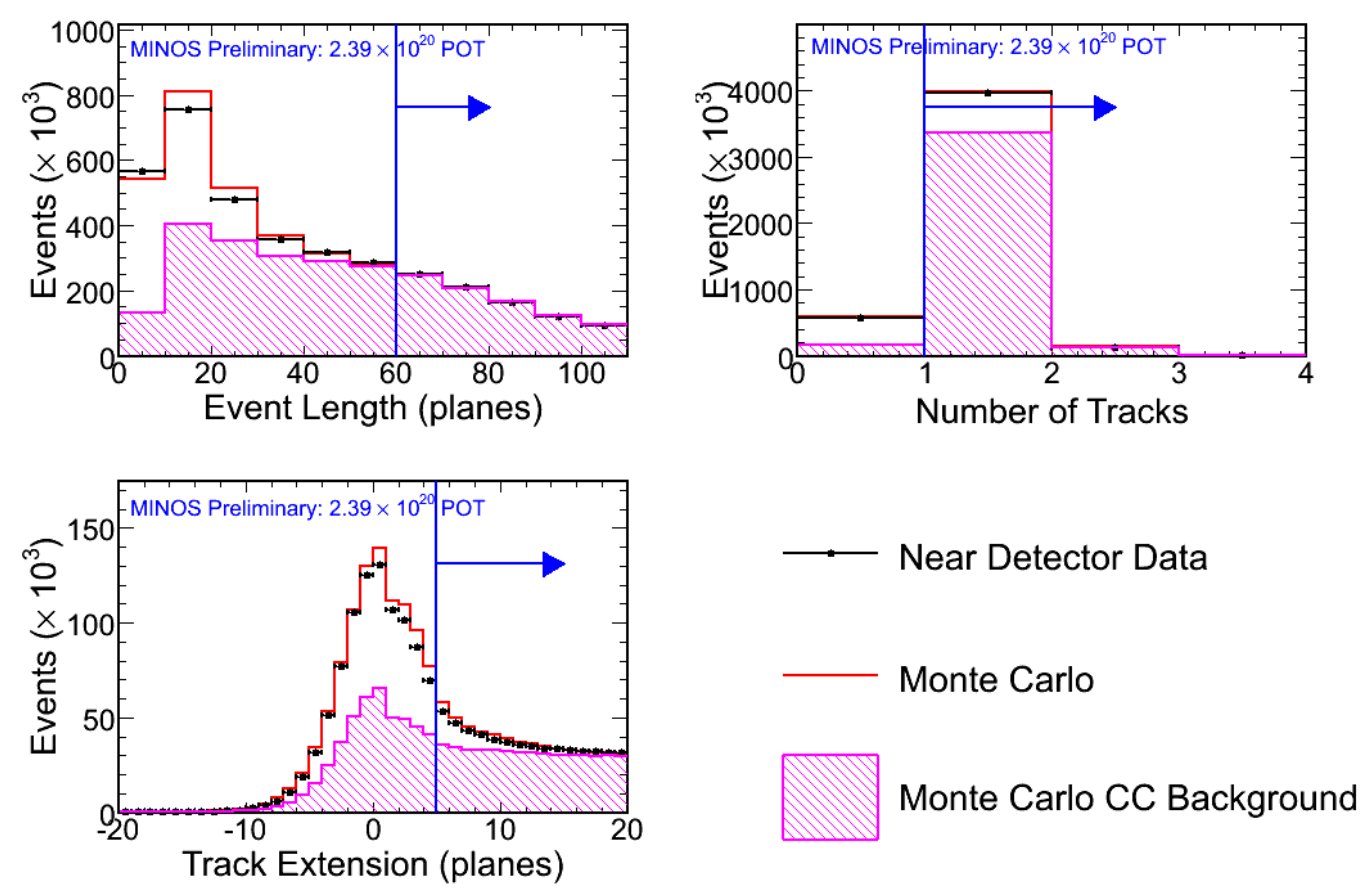

Figure 5.8: Near Detector distributions of the three NC/CC separation variables for both Monte Carlo and data. The blue vertical lines demarcate the values, where events away from the arrow are classified as NC-like.

II. Events crossing $<60$ planes which do not have a reconstructed track are classified as NC-like.

III. Events crossing $<60$ planes containing both a reconstructed shower and track are classified as NC-like based upon the track extension variable, $l$. Events which contain a track that protrudes $<5$ planes out of the shower are classified as NC-like.

IV. Events crossing $<60$ planes which are not classified as NC by the previous cuts are classified as CC-like using the MINOS charged-current PID[71] or discarded from the analysis.

The power of the separation variables are individually plotted in Fig. 5.8. The Near Detector energy spectrum of the NC separated events is shown in Fig. 5.9

The CC PID is a likelihood constructed from a combination of three probability 


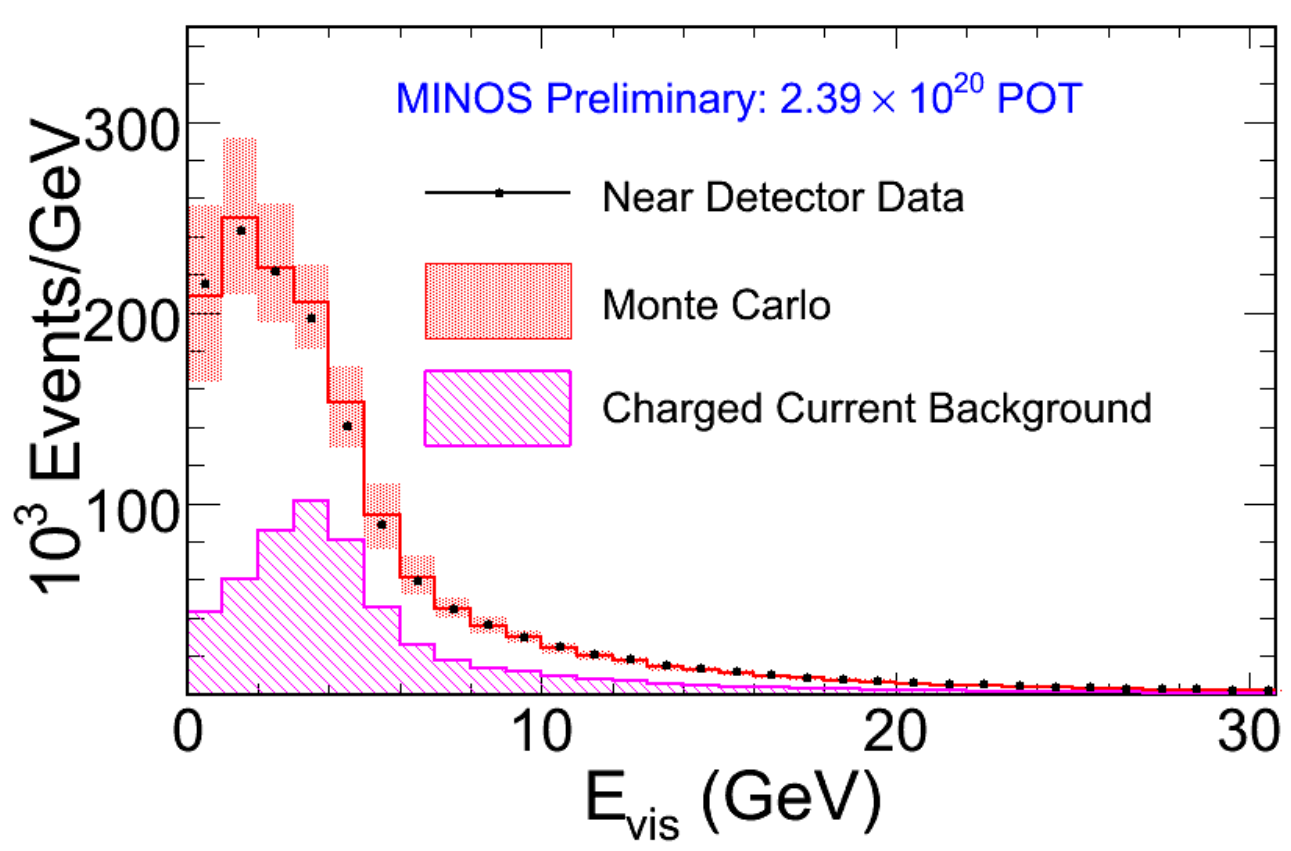

Figure 5.9: The RunI and RunII ND data after all cuts as well as NC/CC event separation. The data points (black) are well within the Monte Carlo error bars (red), which is dominated by hadron production and cross-section uncertainty. Because of the high event rate per POT in the Near Detector, the statistical error bars on the black data points are imperceptible. The Monte Carlo illustrated is generated from a different exposure of POT than the actual data, and is then scaled to data POT. 
density functions (PDF) that makes use of the topological differences between a CC and NC event: long muon track versus short diffuse hadronic shower. The three PDFs are the track pulse height fraction, pulse height per plane, and event length. The separate probabilities for charged and neutral-current classification determined by the CC PID are given by

$$
\begin{aligned}
& P_{C C}=\prod_{i=1}^{3} P D F_{C C_{i}}, \\
& P_{N C}=\prod_{i=1}^{3} P D F_{N C_{i}} .
\end{aligned}
$$

The CC PID parameter itself is then a convolution of the different probabilities designed to range from zero to one given by

$$
P I D=\sqrt{-\log P_{N C}}-\sqrt{-\log P_{C C}}
$$

\subsection{Neutral-current Extrapolation to Far Detector}

The predicted energy spectrum at the Far Detector is achieved by extrapolation from the Near Detector spectrum. The need for an extrapolation in predicting the Far Detector neutrino energy spectrum stems in large part from the intrinsic uncertainty in important Monte Carlo quantities, such as neutrino cross-section and hadron production. A significant strength of the MINOS experiment is that a two detector design allows the Near Detector data to be used to make the Far Detector prediction, thereby unencumbering the prediction from the full extent of Monte Carlo uncertainties. The primary extrapolation for the $\mathrm{NC}$ analysis is the 'far over near' $(\mathrm{F} / \mathrm{N})$ method, where the cross-checking of the extrapolation is done using an implementation of the Marquardt fitting technique[81]. The following chapter 
details the work undertaken in constructing and refining the F/N method.

The discussion begins with the F/N method itself and the implementation in the NC analysis coding framework, NCUtils. The systematic uncertainties affecting the NC analysis will be presented, as will results from Monte Carlo studies and pseudo-experiments.

\subsubsection{Far Over Near Extrapolation}

The F/N extrapolation is the method used by the Neutral-current analysis to predict the Far Detector energy spectrum. It uses the ratio of the Far and Near Detector Monte Carlo in each bin of energy, multiplied by the number of events in the Near Detector data for the prediction. The highlights of the F/N method are that it requires minimal computation time and is robust to energy dependent biases in the MC.

In making the prediction, the extrapolation is a composite of not only the true $\mathrm{NC}$ events, but also the background events and addresses the ways in which the backgrounds change between the Near and Far Detector. The F/N extrapolation can be algebraically written as

$$
F D_{i}^{\text {predicted }}=N D_{i}^{\text {data }}\left(\frac{\sum_{j} F D_{i j}^{M C} P\left(v_{\mu} \rightarrow v_{[\mu, \tau, e]}, E_{j}\right)}{N D_{i}^{M C}}\right)
$$

where $N D_{i}^{d a t a}$ is the number of neutrinos in the Near Detector in the $i($ th) bin of reconstructed energy, $N D_{i}{ }^{M C}$ is the number of neutrinos measured in the Near Detector Monte Carlo in the $i\left(\right.$ th) bin of reconstructed energy, $F D_{i j}{ }^{M C}$ is the number of neutrinos measured in the Far Detector Monte Carlo in the $i($ th) bin of reconstructed energy and $j($ th) bin of true energy, $E$ is the true neutrino energy and $P$ the probability of muon neutrino transition to $v_{\mu}, v_{\tau}$ or $v_{e}$. 
The Far Detector MC is generated with no oscillations, but the extrapolation must take oscillations into account because a large portion of the background to the NC sample is misidentified $v_{[\mu, e, \tau]} \mathrm{CC}$ events. Therefore, in the extrapolation the $\nu_{\mu}$ in the NuMI beam are oscillated, as a function of the neutrinos true energy, and integrated in bins of reconstructed energy to account for the changing background.

Before the extrapolation can be performed, the MC for both detectors must be separated into their constituent components of neutrino flavor and scaled to match the POT exposure of the data. Although eq. (5.9) highlights the $v_{\mu}$ transitions, there remains one other group of events that act as background to the NC signal; intrinsic beam $v_{e}$ generated at Fermilab in the NuMI beam. Addition of the signal $v_{\mu}$ NC events to the backgrounds makes five separate MC data sets $\left(\mathrm{v}_{\mu} \mathrm{CC}, \mathrm{v}_{\tau} \mathrm{CC}, \mathrm{v}_{e} \mathrm{CC}\right.$, beam $v_{e}$ and signal $v_{\mu} \mathrm{NC}$ ) that go into the extrapolation to generate a predicted $\mathrm{NC}$ spectrum after separation. The MC is scaled to match the POT exposure of the data acquired. The Far Detector MC used in the NC analysis is generated using a high POT exposure, $e>1 \mathrm{e} 23$ POT, which provides statistical coverage in low event rate areas and allows studies to proceed using MC as 'fake' data which preserves the blinding process.

The MC data from the five classes of events are used to construct individual 2D histograms of true vs. reconstructed energy, shown in Fig. 5.10(a), where Fig. 5.10(b) illustrates the spread of true neutrino energy values for a single bin of reconstructed energy. Using the assorted $2 \mathrm{D}$ histograms, all the events in an individual bin of true energy are multiplied by the same survival or transition probability. After applying the oscillations, the MC is converted to a reconstructed energy spectrum by integrating across all the true energy bins for each bin of reconstructed energy, producing the quantity $\sum_{j} F D_{i j}{ }^{M C} P\left(v_{\mu} \rightarrow v_{x}, E_{j}\right)$ in eq. 5.9 . The reconstructed energy spectrum for each separate data set, both for signa and mis-identified ackgrounds, are added together into one spectrum. The number of 


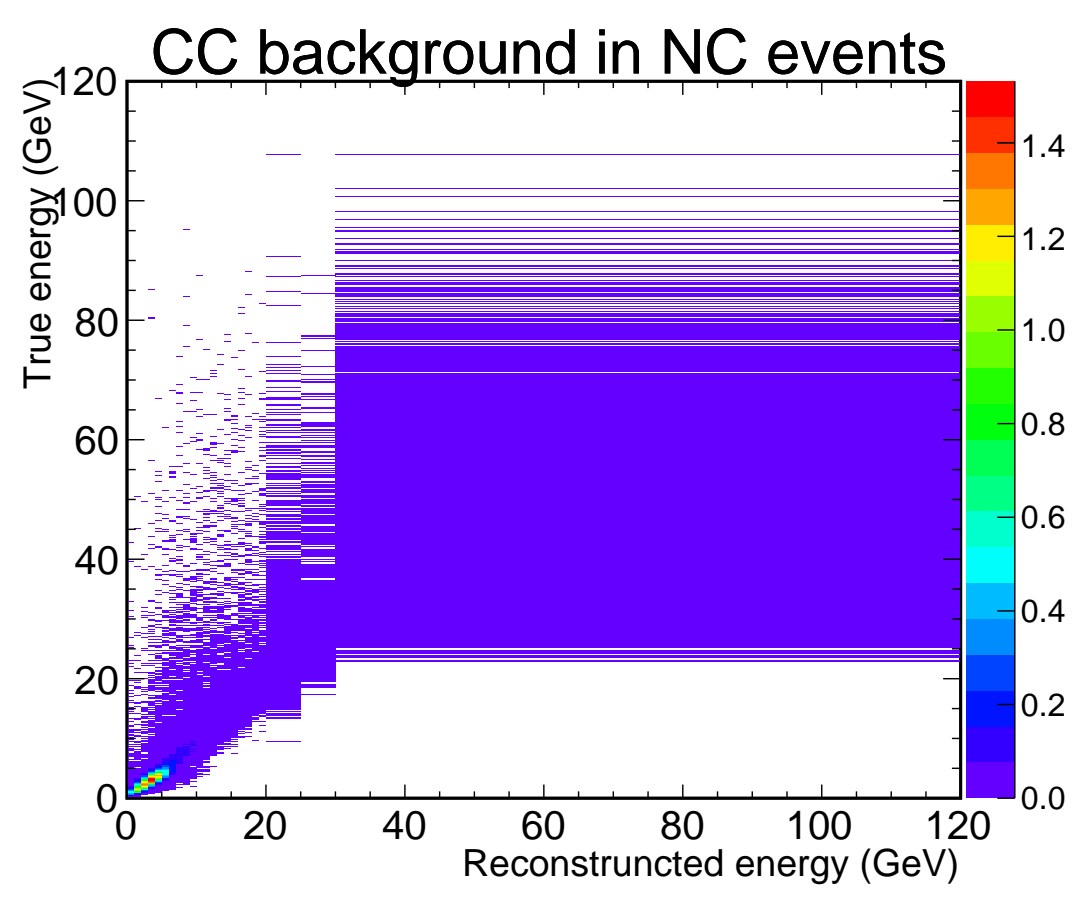

(a)

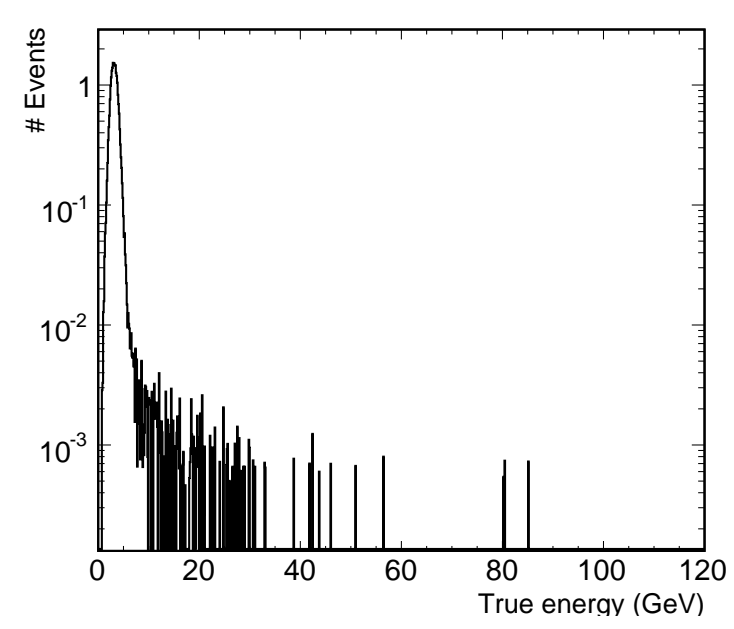

(b)

Figure 5.10: 2D Histogram (a): Monte Carlo true vs. reconstructed energy histogram of the background $v_{\mu}$ charged-current events in the neutral-current event sample. Histogram (b) shows the 1D true energy projection of the 3-4 GeV bin of reconstructed energy. The neutrino survival probability is applied in horizontal slices of (a); in terms of true neutrino energy. The integral of the projection (b) gives the FD MC events in the 3-4 GeV bin after oscillation and POT normalization. 


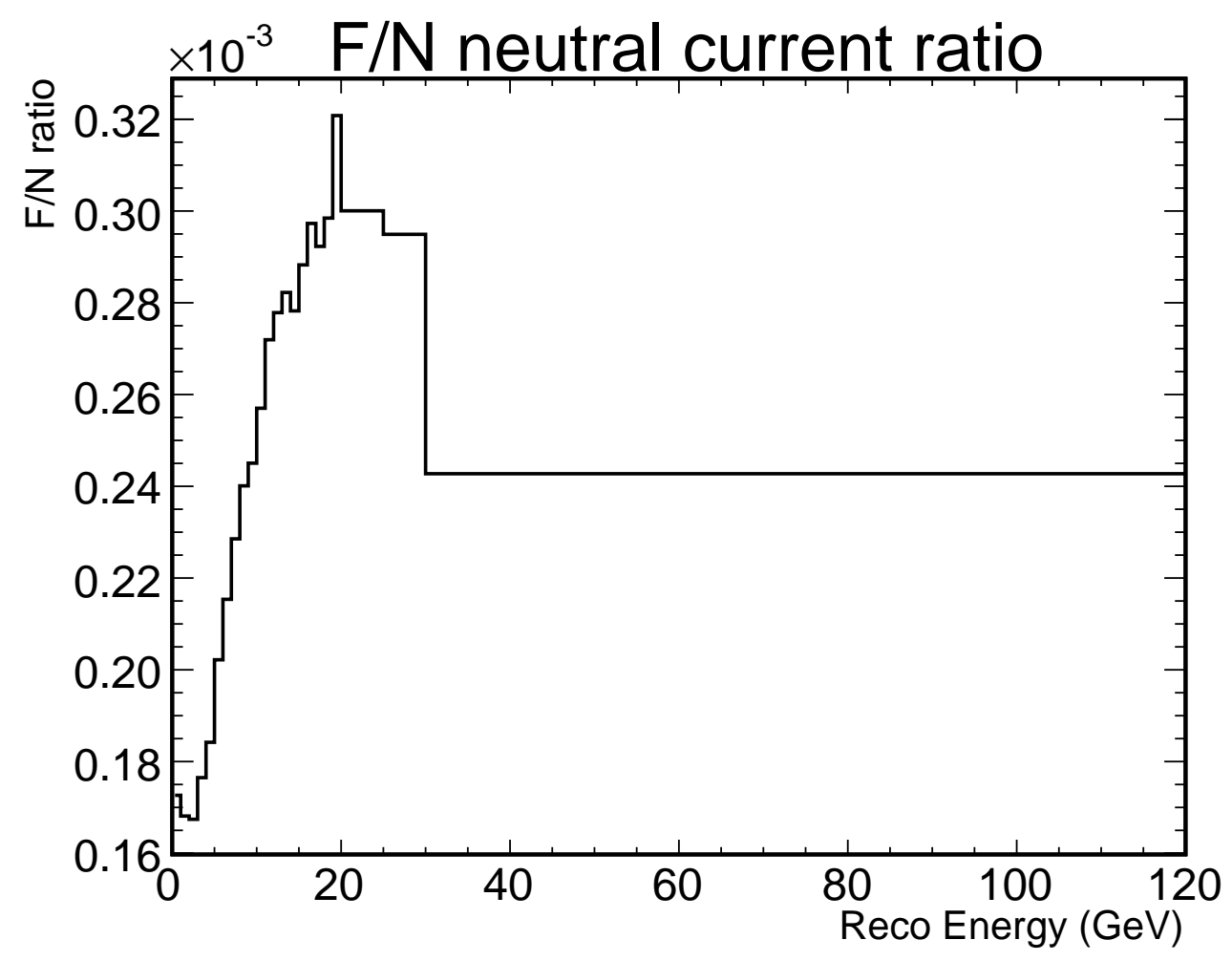

Figure 5.11: F/N ratio for the extrapolation of the NC events from the Near Detector to the Far Detector. The scale of the ratio represents the orders of magnitude difference between the number of events recorded in the Near Detector versus the Far Detector for the same bin of energy. The variable bins of reconstructed energy are also clearly represented: $1 \mathrm{GeV}$ increments for $0-20 \mathrm{GeV}, 5 \mathrm{GeV}$ increments for 20-30 and a single $90 \mathrm{GeV}$ bin for $30-120 \mathrm{GeV}$.

events in each bin of reconstructed energy is then divided by the number of events in the corresponding energy bin in the Near Detector MC to produce the $\mathrm{F} / \mathrm{N}$ ratio, shown in Fig. 5.11. Reflected in the F/N ratio is the POT scaling of both the FD MC to FD data as well as the ND MC to ND data. Beyond the POT scaling, the ratio shows that the number of events in the Near Detector is orders of magnitude larger than the number of events in the Far Detector for the same exposure. With the scaling and F/N ratio calculated, a FD neutrino energy spectrum prediction is the bin by bin product of the F/N ratio and the ND data.

Because neutrinos oscillate as a function of their true neutrino energy, there is a concern that applying the same survival probability to every event in a bin of 
true energy will introduce a systematic uncertainty to the prediction. However, the width of the true energy bins is small enough, $0.12 \mathrm{GeV}$, that no measurable energy shift is introduced in approximating all the neutrinos in a single bin as having the same energy. Along with the bin width, the use of a high exposure MC sample reduces error stemming from applying the survival probabilities in a bin-by-bin fashion.

\subsubsection{Optimization}

The F/N method creates a specific predicted FD spectrum using a specific list of input parameters. With each prediction, a comparison is made to data using a $\chi^{2}$ statistic:

$$
\chi^{2}=\sum_{i} 2\left(\left(\text { expected }_{i}-\text { observed }_{i}\right)+\text { observed }_{i} * \log \left(\frac{\text { observed }_{i}}{\text { expected }_{i}}\right)\right)
$$

A minimizing routine uses the $\chi^{2}$ statistic to 'swim' towards the values producing the greatest data/MC agreement using the $\mathrm{F} / \mathrm{N}$ extrapolation method during each iteration to produce the predicted spectrum. The NC analysis currently uses the MINUIT minimizing routine[82] to find the best fit values.

\subsection{Systematic Uncertainties}

The systematic uncertainties for the NC analysis are the result of event selection, reconstruction and simulation of neutrino events. In order to gauge the magnitude of each systematic, tests are conducted to measure the deviation in best-fit parameters from the $\mathrm{F} / \mathrm{N}$ method using a Monte Carlo set that is altered by the $1 \sigma$ value of a specific systematic uncertainty, while keeping the rest unaltered.

The following sections look at each systematic uncertainty that contributes to the NC analysis, explains the effect on the data, and lists the size of the ensuing 


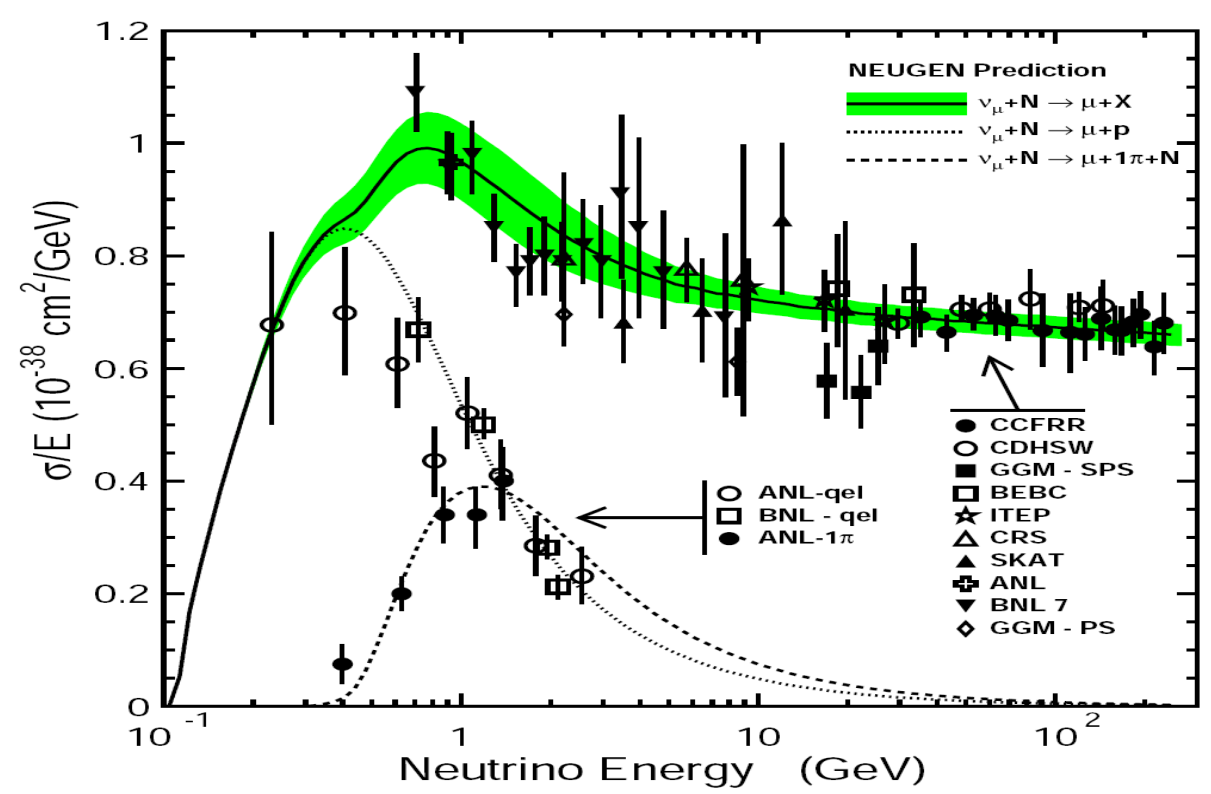

Figure 5.12: NEUGEN-V3 calculation of the muon neutrino charged-current crosssection. The quasi-elastic cross-section is denoted by the dotted line, while the resonance, or single pion, is the dashed line. The solid black line denotes the total cross-section where deep inelastic scattering dominates at energies $>\sim 5 \mathrm{GeV}$. The shaded regions represent the NEUGEN cross-section uncertainties.

uncertainty.

\subsubsection{Neutrino-Nucleus Interaction}

MINOS uses the NEUGEN neutrino-nucleus interaction generator[83] to simulate the cross-sections, shown in Fig. 5.12, and intranuclear rescattering of a neutrino collision. Important to the cross-sections are the poorly measured values of the axial vector mass, $M_{a}$, for both resonant (RES) and quasi elastic (QEL) events. The $M_{a}$ parameter contributes to the size of the cross section and the $M_{a}^{R E S}$ value is $1.12 \pm 0.1485$ and $M_{a}^{Q E L}$ is $0.99 \pm 0.168$. Both $M_{a}^{Q E}$ and $M_{a}^{R E S}$ are combined to produce one parameter known as the charged-current axial vector mass (CCMA), with a single associated systematic uncertainty.

There is a further set of parameters in the NEUGEN generator that are used to adjust the cross section of deep inelastic scattering events(DIS). These parameters, 
known as $k n o_{i j k}$, are values used to scale the exclusive DIS cross-section channel relating to the various neutrino nucleon interaction possibilities, where the components of the total cross section are defined as

$$
\sigma_{\text {total }}=\sigma_{Q E L}+\sum_{k}\left(\sigma_{\text {resonance }}^{k}+k n o_{i j k} \sigma_{D I S}^{k}\right)
$$

The NC analysis is only sensitive to two of the $k n o_{i j k}$ parameters: $k n o_{112}$ and $k n o_{122}$. The subscripts on the parameters relate to possible interaction types where $i=1$ is for CC interactions, $j=1$ is for $v-p$ interactions, $j=2$ is for $v-n$, and $k=2$ is the final state multiplicity. The uncertainty on $k n o_{112}$ is $0.1 \pm 0.33$ and $k_{n o} 122$ is $0.3 \pm 0.33$.

\subsubsection{Beam Modeling}

The uncertainty in the modeling of the beam is the cumulative effect of uncertainties related to beam systematics, hadron production and detector parameters. These three sets of beam uncertainties provide the knobs for the SKZPTV beam parameterization to improve the data/MC agreement. All the SKZPTV parameters are adjusted to their $\pm 1 \sigma$ values in unison to produce the beam modeling uncertainty as discussed in Sec. 3.3.

\subsubsection{Hadronic Energy}

There are two issues related to the hadronic energy determination when comparing NC events between the Near and Far Detector: 1) the absolute hadronic energy resolution and 2) the relative difference in hadronic energy resolution between the two detectors. The uncertainty related to the absolute hadronic energy is produced by examining data from the Calibration Detector, as well as looking at intranuclear effects. 
The MINOS Monte Carlo is tuned to the CalDet data so that the energy response in the detector matches the known energy of the incoming particle (pion or proton). The uncertainties remaining after tuning are listed in table 5.1, and are conservatively added in quadrature to get $5.6 \%$ uncertainty for the absolute hadronic energy scale from calibration.

\begin{tabular}{|l||c|}
\hline \multicolumn{1}{|c||}{ Source } & Energy Scale Uncertainty \\
\hline Tuning hadron(pion) MC to CalDet data & $2.5 \%$ to $5 \%$ (energy dependent) \\
CalDet beam energy uncertainty & $2 \%$ \\
CalDet stopping-muon calibration uncertainty & $1.4 \%$ \\
Tuning stopping-muon MC to data & $1.2 \%$ \\
\hline
\end{tabular}

Table 5.1: Absolute energy scale systematics errors.

Beyond calibration the absolute hadronic energy scale is affected by the uncertainty in the hadronization model and intranuclear effects. NEUGEN-V3 allows hadrons to interact while exiting the incident nucleus, known as "final state interactions", and ultimately this behavior affects the visible energy of the constituents in the hadronic shower[84]. The significant uncertainties in hadron-nucleon interactions are related to the pion behavior and the rate of absorption and energy transfer to nucleon clusters. The contribution of these uncertainties to the absolute hadronic energy scale are calculated by comparing the reconstructed shower energy when the intranuclear rescattering is turned "on" to the shower energy when the simulation is turned "off". This represents the only method to address the two extrema of nuclear re-interaction. There is a $8 \%$ change in the shower reconstructed energy, which coupled with the $5.6 \%$ change from the calibration results in an overall uncertainty in the absolute hadronic energy scale of $10 \%$.

The relative uncertainty in energy resolution between the Near and Far Detector is calculated using a Monte Carlo comparison to both data sets. The uncertainty added in quadrature of the shower energy scale between the MC-ND data and MCFD data results in a total relative uncertainty of $3 \%$. 


\subsubsection{Normalization}

The uncertainty in the amount of neutrinos expected at the Far Detector is especially important because the $\mathrm{NC}$ analysis is a disappearance search.

Large contributors to this uncertainty are the livetime of the detector as well as the fiducial mass. The uptime of the Far Detector is now stable at $>99.5 \%$ while beam is being supplied from NuMI, but during the duration of data taken for the $\mathrm{NC}$ analysis this was not always the case. This contributed $1 \%$ to the uncertainty in the overall normalization. The fiducial volume uncertainty is drawn from the steel in the Far Detector planes, which was drawn from various batches or 'heats' and rolled into 0.5 " slabs. While the density difference across heats is small, the combination of width irregularity contributes a $2 \%$ uncertainty to the total normalization uncertainty from the fiducial mass.

The reconstruction efficiency or bias can be tested by alterations to the level of noise and light associated with an event. In this scheme, the first test is to increase the noise level by a factor of two and then decreased to zero, and compare the number of events to nominal noise. The second test is to increase the light level by $\pm 5 \%$ in the detector simulation. The resulting difference of reconstructed events was $0.5 \%$.

The total uncertainty to event normalization is the sum in quadrature of individual sources, producing a value of $2.3 \%$. The effect of the normalization is one of the most important for an oscillation search and to ensure that no underestimate of this particular systematic uncertainty took place a conservative estimate on the uncertainty for the normalization is set at $4 \%$ for the $\mathrm{NC}$ analysis. 


\subsubsection{Charged-Current Background in the Neutral-Current}

\section{Sample}

The selected sample of NC events contains both true NC as well as background $v_{\mu}$ CC events that are selected as NC. A well constrained value of the $v_{\mu}$ background is essential for an accurate Far Detector prediction because of oscillation. Over the $735 \mathrm{~km}$ beam path, a deficit in the rate of $v_{\mu}$ has been observed[71], creating proportionally less background CC events at the Far versus Near Detector. An unconstrained uncertainty on the amount of $\mathrm{CC}$ background will cause a distortion in any predicted Far Detector spectrum, which would dramatically reduce sensitivity to sterile neutrinos. Not only does the $\mathrm{v}_{\mu} \mathrm{CC}$ background change between detectors, it also constitutes the largest background to the NC selected sample. To gauge the size of the background, as well as the uncertainty, the 'alternate current' method utilized Monte Carlo and data from the various beam configurations.

The alternate current method is a data-driven approach to obtain the $\mathrm{CC}$ contamination in the $\mathrm{NC}$ sample by looking at both the $\mathrm{NC}$ and $\mathrm{CC}$ event ratio between different beam configurations and the standard LE010z185i configuration ${ }^{3}$ In each bin of energy the neutrino sample is a combination of both the true NC and background $v_{\mu} \mathrm{CC}$ events,

$$
N^{L E}=N_{c c}^{L E}+N_{n c}^{L E}
$$

where $N^{L E}$ is the total number of neutrinos in the LE configuration, $N_{c c} L E$ the number of CC events and $N_{n c}{ }^{L E}$ the number of NC events. The total number of neutrinos in each bin of energy for an alternate horn current can be calculated by scaling the number of $\mathrm{CC}$ and $\mathrm{NC}$ events in the LE,

$$
N^{\text {alt }}=r_{c c} N_{c c}^{L E}+r_{n c} N_{n c}^{L E}
$$

\footnotetext{
${ }^{3}$ The LE010z185i NuMI beam configuration will be referred to as the LE configuration for the remainder of the section
} 
where $r_{c c}=N_{c c}^{a l t} / N_{c c}^{L E}$ and $r_{n c}=N_{n c}^{a l t} / N_{n c}^{L E}$, both calculated from Monte Carlo.

When the MC values $N^{\text {alt }}$ and $N^{L E}$ are compared to data via a double ratio, defined as

$$
\mathcal{R}=\frac{N_{\text {data }}^{L E} / N_{\text {data }}^{\text {alt }}}{N_{M C}^{L E} / N_{M C}^{\text {alt }}},
$$

the $r$ values illuminate the accuracy with which the Monte Carlo reflects the amount of $\mathrm{CC}$ and $\mathrm{NC}$ events in the data. Use of a double ratio removes any contributing uncertainties from cross-section, flux or other energy dependent uncertainties and isolates the $r$ values as being the only source of uncertainty. The uncertainty from the double ratio $\mathcal{R}$ is then solely the providence of the uncertainty in the $r$ values, which in turn are reflections on how well the NC and CC fraction are known.

While the uncertainty in $\mathcal{R}$ in eq. (5.14) is for the total number of events, the value is attributed to both the ratios of $\mathrm{NC}$ and $\mathrm{CC}$ events for lack of further information that decouple the two. The final CC background uncertainty is then the POT weighted average of 3 alternate beam configurations compared to the LE beam, where one such comparison is shown in the Fig. 5.13. The alternate current method shows a $\sim 12 \% \mathrm{CC}$ background uncertainty across all neutrino energies, where a final and conservative value of $15 \%$ was selected. The increased uncertainty at higher values of reconstructed energy, seen in Fig. 5.13, motivated the more conservative value for the amount of CC background.

\subsubsection{Event Selection}

The cuts designed to accept/reject quality neutrino events have the potential to contribute systematic uncertainties in the amount of events selected. Of all the cleaning cuts applied to the data, the minimum number of strips cut in Sec. 5.2.1 is the only non-negligible contribution to the uncertainty. While the minimum strip cut provides the only significant uncertainty contribution, it is considered in combination with fiducial volume and noise for completeness. As shown in Table 5.2, changing 

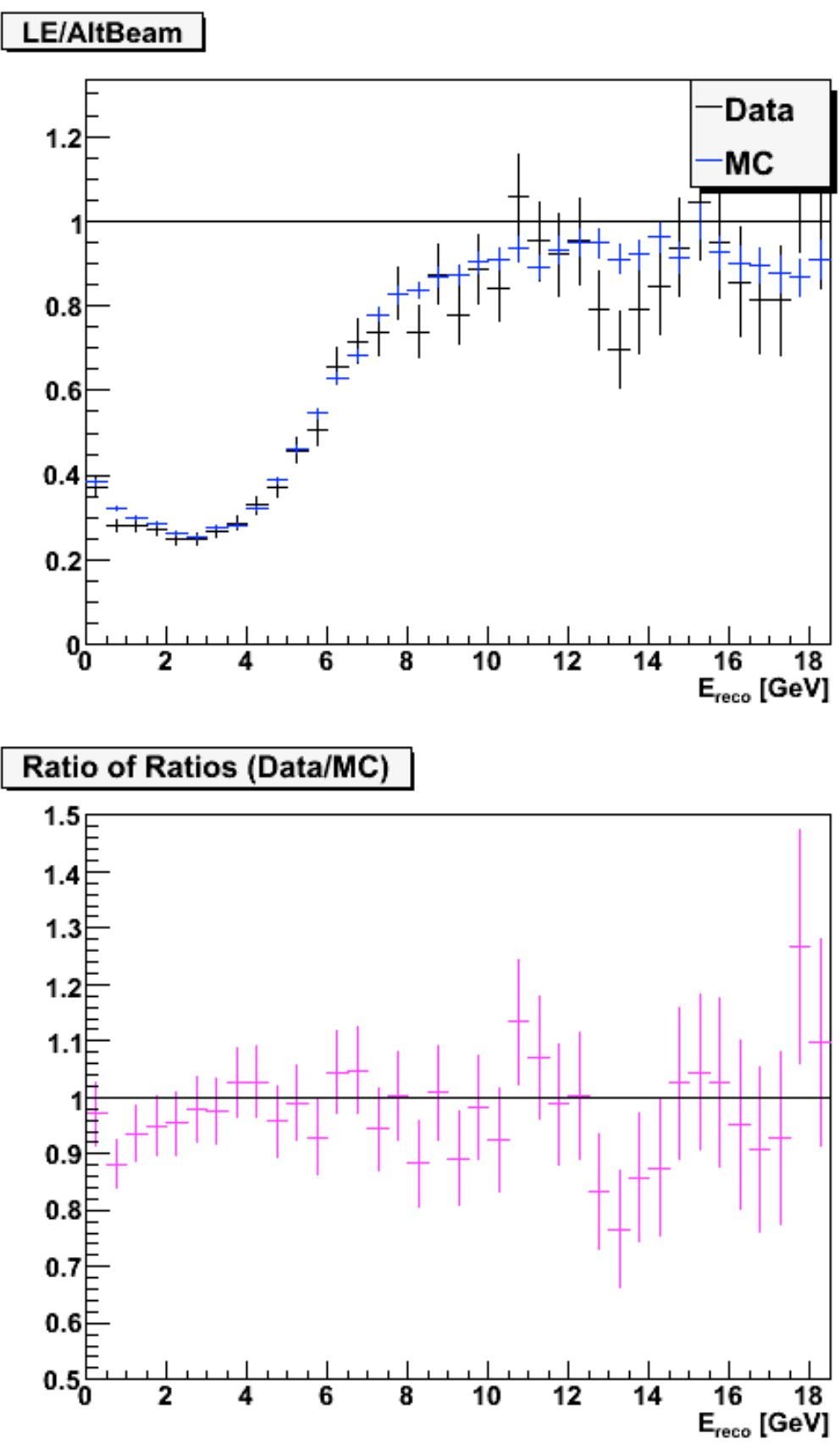

Figure 5.13: The top plot shows the data and MC ratios of the LE divided by alternate beam configuration $N^{L E} / N^{l e 010 z 000 i}$, in this case the same target position as the LE but with no current to the magnetic horns. The number of events used were normalized to protons on target, where the LE Monte Carlo/data exposure was 7.8e19/1.38e 19 and le010z000i was $0.28 \mathrm{e} 19 / 3.29 \mathrm{e} 19$. The difference is illustrated by the size of the respective errors bars. The bottom plot shows the double ratio value $\mathcal{R}$ from eq. (5.14) for the same two beam configurations. 


\begin{tabular}{|c|c|}
\hline Energy Range (GeV) & Uncertainty \\
\hline $0-0.5$ & $15.2 \%$ \\
$0.5-1$ & $2.9 \%$ \\
$1-1.5$ & $0.4 \%$ \\
$1.5+$ & $0 \%$ \\
\hline
\end{tabular}

Table 5.2: Event number uncertainty stemming from the event selection criteria.

all three cuts by their $1 \sigma$ values predominantly affects the number of low energy events selected.

\subsubsection{Electron Appearance Background}

While the NuMI beam begins with a nearly pure beam of $v_{\mu}$, oscillation over the $735 \mathrm{~km}$ baseline changes the composition of neutrinos. The background contribution from the $v_{\mu}, v_{\tau}$ and $v_{e}$ CC events is therefore subject to the likelihood of a $v_{\mu}$ to oscillate into the aforementioned flavors. The particulars of oscillations are governed by a neutrino model where the degree to which the parameters $\Delta \mathrm{m}_{i j}^{2}$ and $\theta_{i j}$ (and their corollary to the mixing matrix elements $\left|U_{\alpha i}\right|^{2}$ for the $3+1$ models) are known has an impact on the anticipated background from charged-current events. Because of the baseline, detector design and muon neutrino beam, MINOS can measure the parameters $\Delta \mathrm{m}_{31}^{2}$ and $\theta_{23}$ via the $v_{\mu}$ charged-current rate and an analysis to measure $\theta_{13}$ is ongoing. In the meantime, the value of $\theta_{13}$ and the other oscillation parameters are taken from complementary solar, reactor and atmospheric neutrino experiments.

The current best limits for the neutrino oscillation parameters are shown in Table 2.1. where the one that makes a significant impact on the NC analysis is value of $\theta_{13}$, which corresponds to $\left|U_{e 3}\right|^{2}$ for the four flavor models. The value of $\theta_{13}$ partially governs the $v_{\mu} \rightarrow v_{e}$ oscillation, and the cause for concern is that $v_{e}$ charged-current events are identified as NC events with a selection efficiency $>95 \%$, see Fig. 5.14 


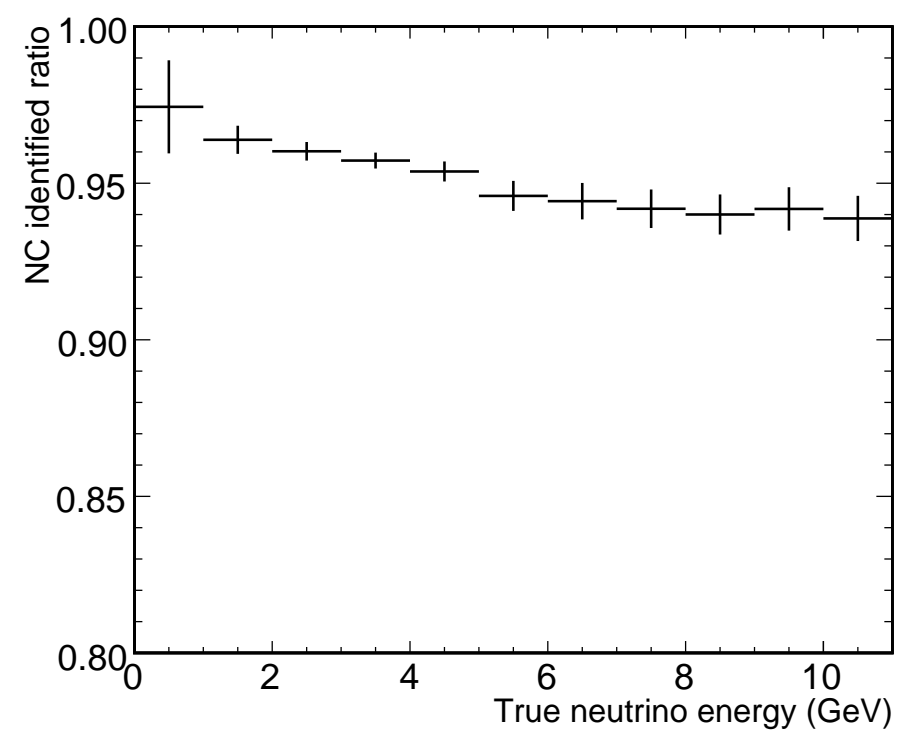

Figure 5.14: Histogram of the efficiency of classifying a true $v_{e}$ charged-current event as a neutral-current event as a function of true neutrino energy. The efficiency remains above $95 \%$ over the $0-5 \mathrm{GeV}$ range where the oscillated and beam $v_{e}$ events have a non-negligible contribution to the NC background.

\begin{tabular}{|c|c|c|c|c|c|}
\hline \multicolumn{3}{|c|}{ 3Flavor } & \multicolumn{3}{c|}{ 4Flavor } \\
\hline \multirow{2}{*}{ Parameter } & \multicolumn{2}{|c|}{ Value } & \multirow{2}{*}{ Parameter } & \multicolumn{2}{c|}{ Value } \\
\cline { 2 - 3 } & $\min$ & $\max$ & & $\min$ & $\max$ \\
\hline$\theta_{13}$ & 0 & 0.21 & $\left|U_{e 3}\right|^{2}$ & 0 & 0.04 \\
$\delta_{C P}$ & 0 & $3 \pi / 2$ & $\delta_{C P}$ & 0 & $3 \pi / 2$ \\
\hline
\end{tabular}

Table 5.3: Values used in calculating the effect of $v_{e}$ appearance. The values in the min column produce no $v_{e}$ appearance, while the max values represent the case of maximum appearance.
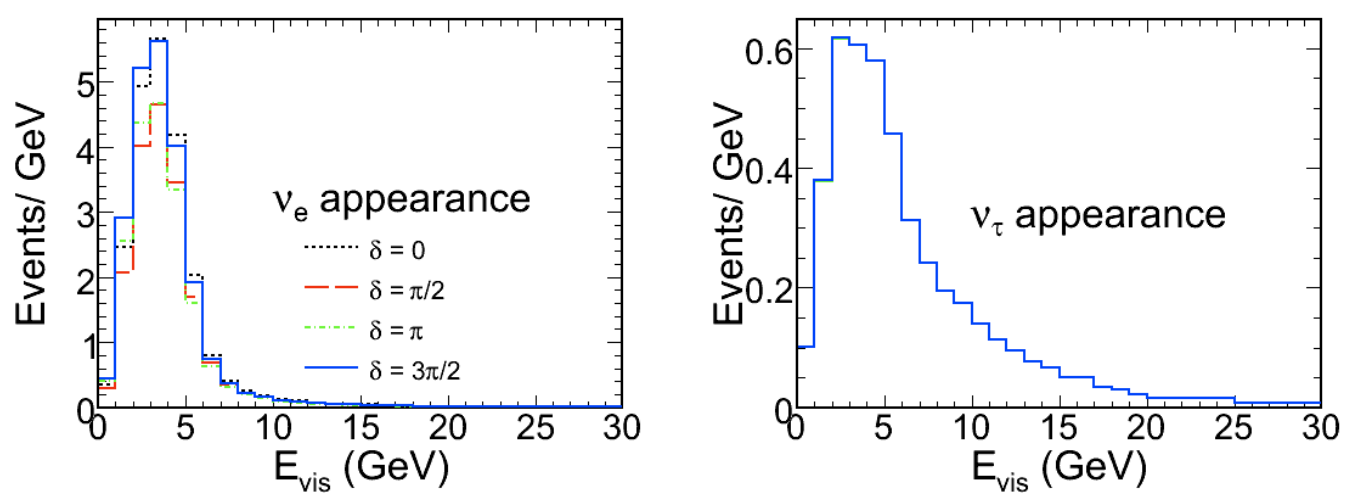

Figure 5.15: Charged-current spectra of the $v_{e}$ and $v_{\tau}$ backgrounds, which change with oscillation. The left plot shows the $v_{e}$ charged-current spectrum setting $\theta_{13}$ to the $\mathrm{CHOOZ}$ limit of 0.21 for different values of the $\mathrm{CP}$ violating phase factor $\delta$. Both spectra are scaled to an exposure of $2.5 \times 10^{20}$ POT. 
In order to address the contribution of an oscillated $v_{e} \mathrm{CC}$ background there are two different scenarios considered: 1) the oscillation parameters are set to values that cause the minimum contribution of oscillated $v_{e}$ events $\left(\delta_{C P}=0\right.$ and $\left.\theta_{13}=0\right)$ and 2) the oscillation parameters are set to the values that cause the maximum contribution $\left(\delta_{C P}=3 \pi / 2\right.$ and $\left.\theta_{13}=0.21\right)$ as summarized in Table 5.3. The oscillated $v_{e}$ spectrum with $\theta_{13}$ set at the CHOOZ limit and maximally enhancing value of $\delta_{C P}$ is shown in Fig. 5.15. The plot also illustrates that the background contribution from $v_{\tau}$ appearance is diminutive in comparison to the $v_{e}$ appearance. Even considering a peak value that is an order of magnitude less than the $v_{e}$ background, the $v_{\tau}$ appearance background is included in the analysis. 


\section{Chapter 6}

\section{Neutral-current Analysis}

The NC analysis uses two different models to test for the existence of a sterile neutrino: the 3Flavor and 4Flavor model. The 3Flavor model uses the current picture of neutrino oscillation covering only the 3 known flavors. An appreciable disagreement between the NC data and a predicted spectrum using the 3Flavor model would be 'suggestive' of a sterile neutrino. The advantages of using the 3Flavor model is that it makes little assumption on the properties of any sterile neutrino, but is not a conclusive test for existence. The 4Flavor model, on the other hand, makes explicit assumptions about the nature of the sterile neutrino, but only for parameter space which coincides with MINOS' sensitivity.

The following section shows the Monte Carlo tests and studies that verify the ability of the NC analysis, and specifically the Far/Near extrapolation method, to establish the existence of a sterile neutrino. The evaluation of the systematic uncertainties will show the effects on the oscillation parameters as well as the uncertainty on the number of neutral-current Far Detector events in MINOS sensitive energy regions. 


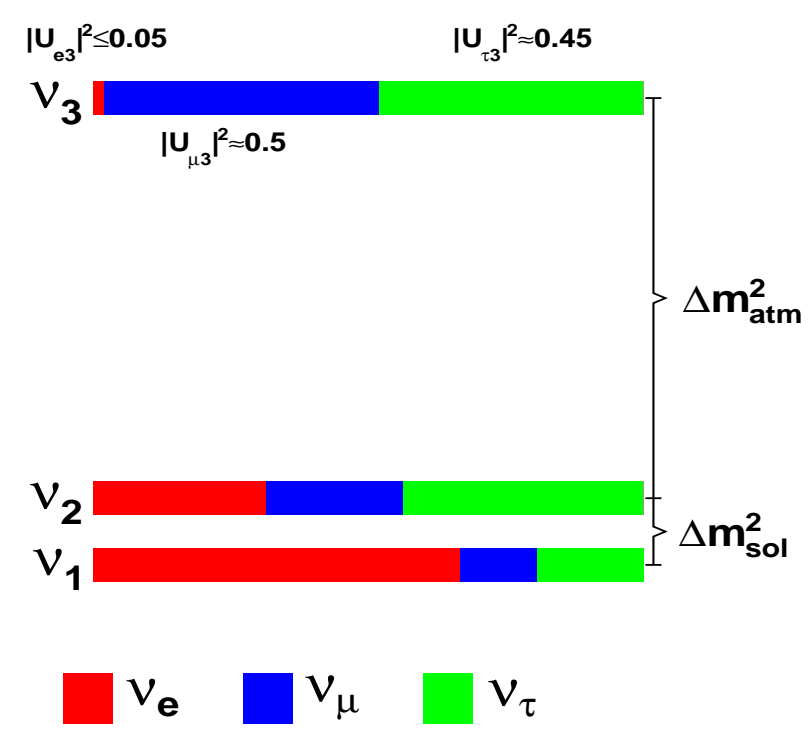

Figure 6.1: Cartoon of the mass squared splittings for the three active neutrinos as well as the respective fractions of $v_{e}, v_{\mu}$ and $v_{\tau}$ in the mass eigenstates $v_{1}, v_{2}$ and $v_{3}$.

\subsection{Flavor Model}

The 3Flavor neutrino model, shown in Fig. 6.1, describes the relationship between the mass and flavor eigenstates as well as the mass squared difference. The first order of testing is to validate that the $\mathrm{F} / \mathrm{N}$ method will reproduce the normal 3 flavor oscillations. Following that will be a discourse on the effect that systematic uncertainties have in shifting the F/N predicted Far Detector spectrum and the corresponding shift in best fit oscillation parameters.

\subsubsection{Initial F/N Validation}

The initial steps of F/N validation is to use Monte Carlo as 'fake' data to check the accuracy with which the F/N method can reproduce the input oscillation parameters. There are two checks:

- V1 - Uses the same FD Monte Carlo for the input into the F/N method and the fake data. 


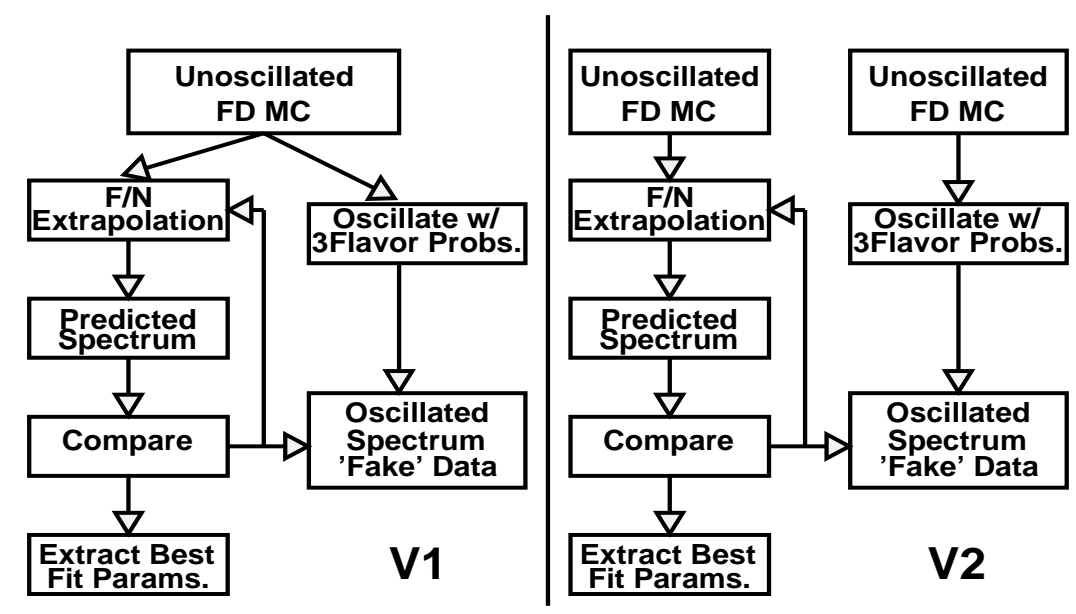

Figure 6.2: Flow diagram of two methods, V1 (left) \& V2 (right), to check the $\mathrm{F} / \mathrm{N}$ method. The V1 method uses the same set of FD Monte Carlo for both F/N prediction and the 'fake' data, removing any statistical variation because the original events are the exact same. The V2 method uses two different sets of FD Monte Carlo in order to test $\mathrm{F} / \mathrm{N}$ methods susceptibility to statistical shifts.

- V2 - Splits the FD Monte Carlo into two distinct sets, where one is used in the $\mathrm{F} / \mathrm{N}$ and the other used as the fake data.

Method V1 and V2 are illustrated in Fig. 6.2.

The V1 method starts by directly oscillating the FD Monte Carlo by user defined parameters and uses this as a fake data set. Then, it uses the same initial unoscillated FD Monte Carlo as input into the F/N method. The F/N method undergoes multiple iterations of producing predicted spectra, while trying to find the oscillation values that minimize the difference between the predicted energy spectrum and the fake data. Because the same FD Monte Carlo is used for both the prediction and fake data, the only difference between the energy spectra comes from the F/N method itself. The results of method V1 are shown in Fig. 6.3, where the input and best fit values are found in Table 6.1.

The V2 method behaves similarly to the V1 with the exception that the FD Monte Carlo is split into two equal sized data sets. One set is used for the fake data, while the other is used as input for the F/N FD Monte Carlo. The V2 method tests the susceptibility of the F/N method to statistical variations. The results using 

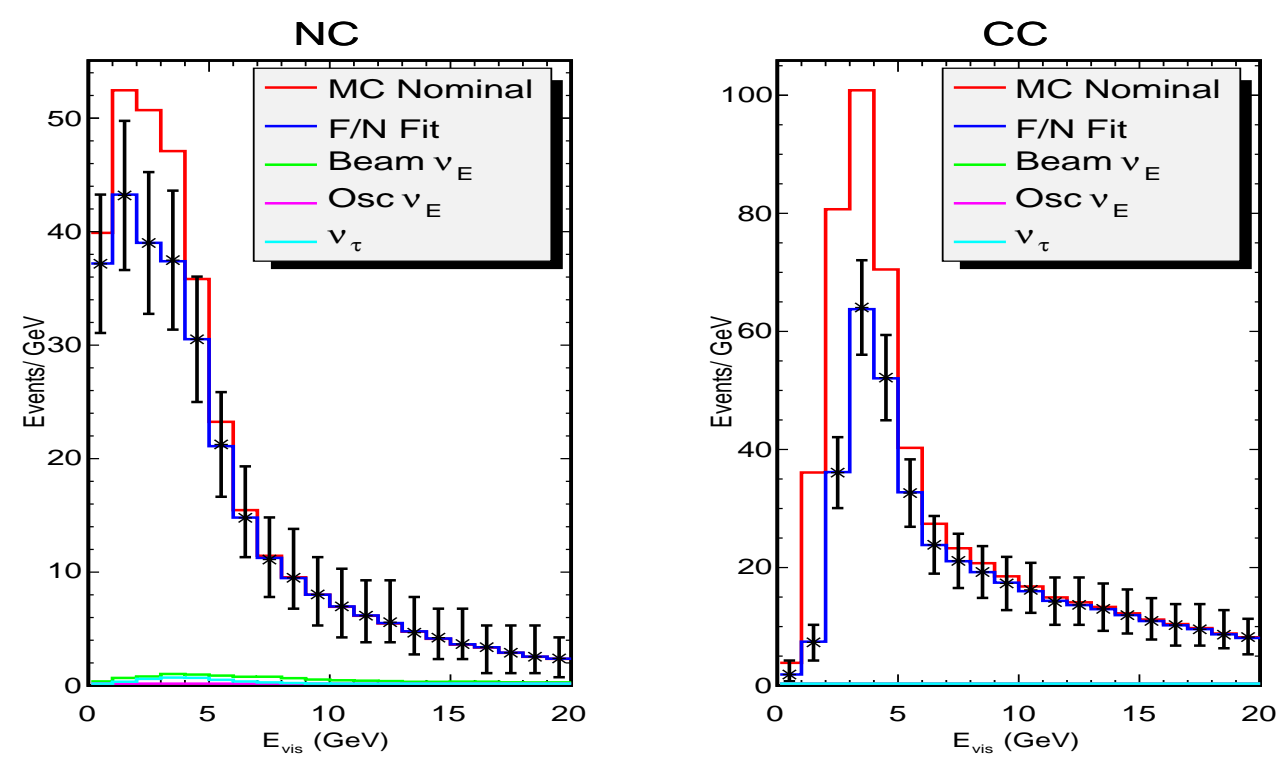

(a)
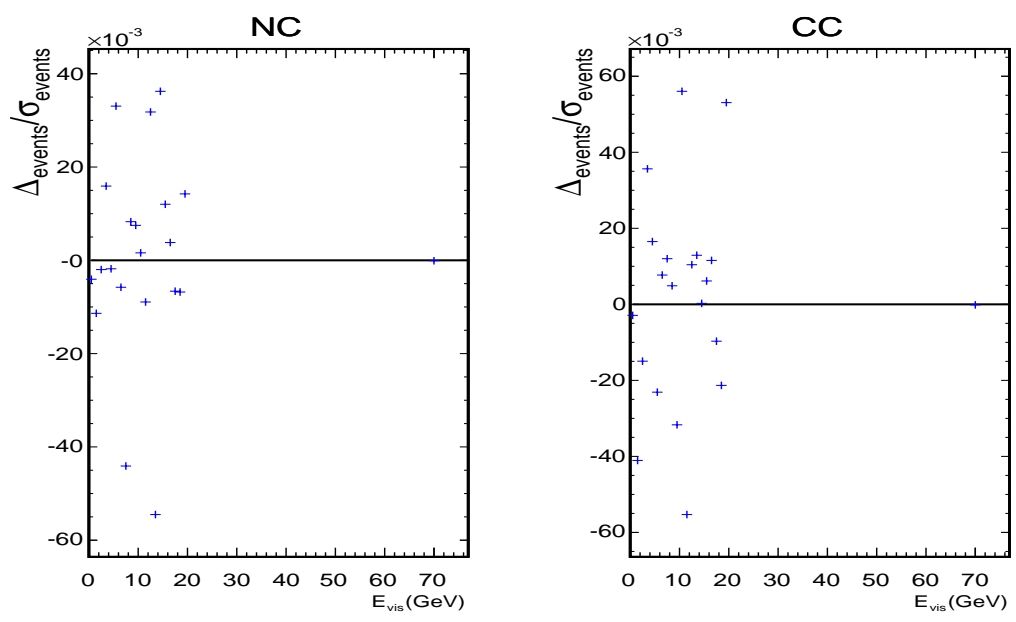

(b)

Figure 6.3: Figure (a) shows the predicted $\mathrm{CC}$ and $\mathrm{NC}$ energy spectrum results of a Monte Carlo test using the 3Flavor model, with $\theta_{13}=0$ and an exposure of $2.5 \times 10^{20}$ POT. The same unoscillated Monte Carlo set was used for both the fake data and the F/N FD Monte Carlo. The difference being that the fake data was oscillated directly event by event while the FitMC results, shown by the blue lines, are the result of the F/N prediction. Figure (b) is the difference between $\mathrm{F} / \mathrm{N}$ prediction and fake date for each bin of energy divided by the statistical uncertainty. 


\begin{tabular}{|c|c|c|c|c|}
\hline \multirow{2}{*}{ Method } & \multicolumn{2}{|c|}{ Input Value } & \multicolumn{2}{c|}{ Best Fit } \\
\cline { 2 - 5 } & $\Delta \mathrm{m}_{31}^{2}\left(10^{-3} \mathrm{eV}^{2}\right)$ & $\theta_{23}$ & $\Delta \mathrm{m}_{31}^{2}\left(10^{-3} \mathrm{eV}^{2}\right)$ & $\theta_{23}$ \\
\hline \multirow{2}{*}{ V1 } & 2.38 & 0.785398 & 2.3826 & 0.785084 \\
V2 & 2.38 & 0.785398 & 2.3824 & 0.751783 \\
\hline
\end{tabular}

Table 6.1: Results from the two different initial methods of validating the $\mathrm{F} / \mathrm{N}$ method. Both tests are done with $\theta_{13}=0$ and an exposure of $2.5 \times 10^{20}$ POT.

the V2 method of validation are shown in Fig. 6.4 and Table 6.1. That the returned values for the oscillation parameters closely match the input values, especially for the V1 method, illustrates a lack of method induced bias in the F/N extrapolation.

Both the V1 and V2 tests show that the F/N extrapolation can accurately and precisely predict the FD energy spectrum and extract the values of the oscillation parameters. Fig. 6.3(a) shows the F/N Fit spectrum following the data. Further supporting the validity of the F/N method is that the discrepancy between the best fit oscillation parameters and true input parameters is partially the result of the optimization process. The MINUIT fitter uses a minimization routine based on the $\chi^{2}$ statistic in eq.5.10, which ends when the difference in $\chi^{2}$ values, $\Delta \chi^{2}$, is small. In a flat region of $\chi^{2}$-space, the $\Delta \chi^{2}$ value may be small enough to stop the minimization process before reaching the true best fit values. The difference is ultimately academic, because the difference in $\Delta \mathrm{m}_{31}^{2}$ and $\theta_{23}$ between the two methods shown in table 6.1 , is small.

\subsubsection{Systematic Uncertainty Shifts}

The work in Sec. 6.1.1 demonstrates that the F/N extrapolation works well in the absence of systematic uncertainty. Including the systematics shows the susceptibility of the F/N method to a shifted energy spectrum. There are in essence two types of systematic uncertainties: those that change the number of events in a given energy bin and those that shift events between energy bins. An uncertainty on the number of events in the bin will translate to an uncertainty in the $\theta_{23}$ value, because 

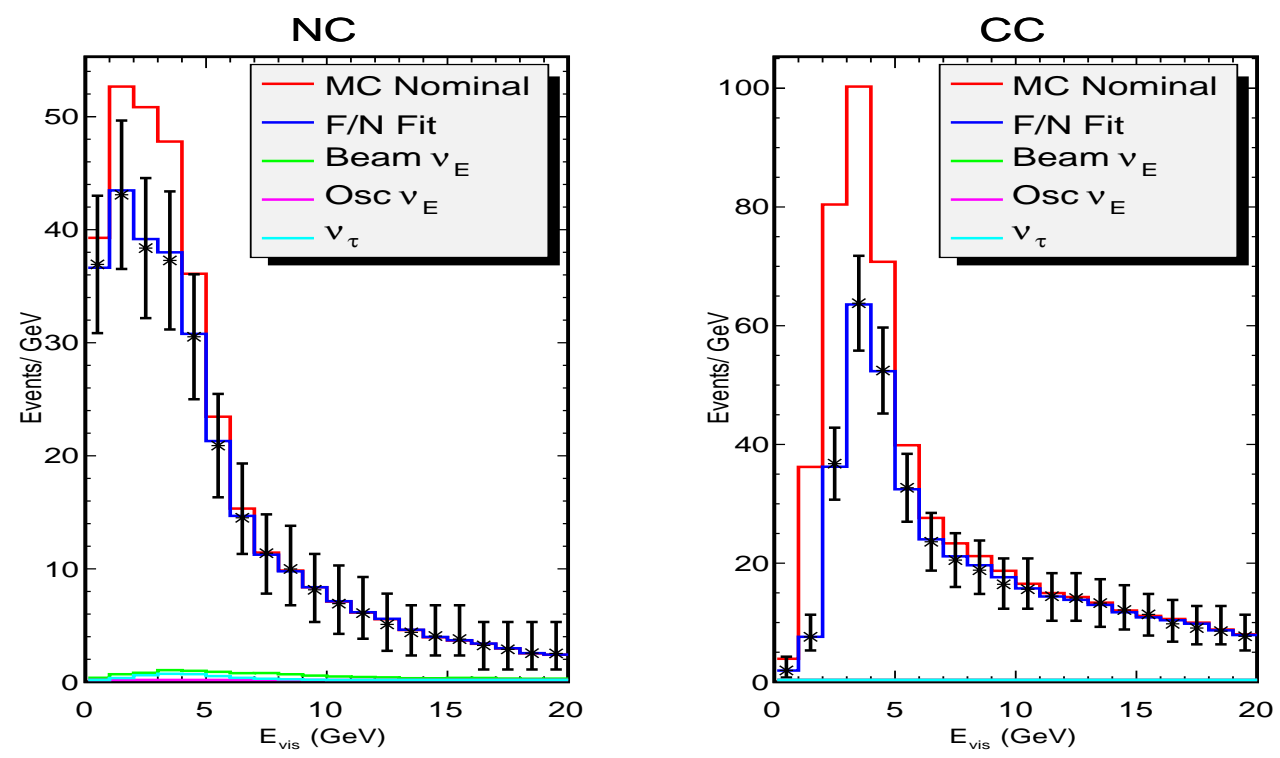

(a)
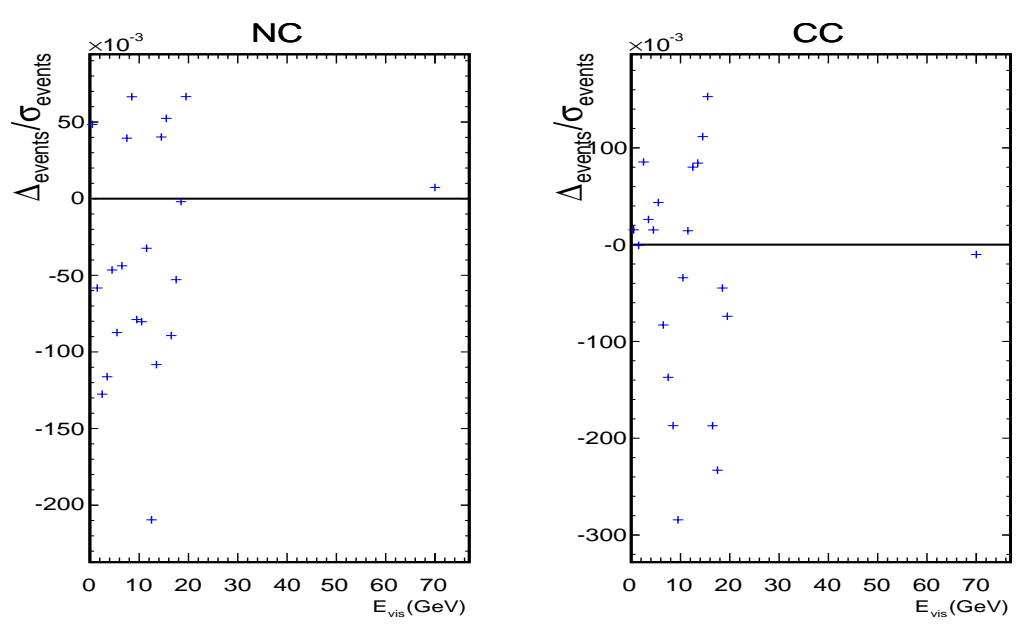

(b)

Figure 6.4: Results of the V2 validation method where figure (a) shows the predicted CC and NC energy spectra results of a Monte Carlo test using the 3Flavor model, setting $\theta_{13}=0$ and an exposure of $2.5 \times 10^{20}$ POT. Figure (b) shows the difference between $\mathrm{F} / \mathrm{N}$ prediction and fake date for each bin of energy divided by the statistical uncertainty, $\Delta_{\text {events }} / \sigma_{\text {events }}$. The V2 method uses two distinct sets of FD Monte Carlo. 
the resolution on the size of the oscillation dip becomes blurred. The location of the oscillation dip determines the $\Delta \mathrm{m}^{2}$ value, so shifting events between bins will contribute an uncertainty to $\Delta \mathrm{m}^{2}$.

To gauge the effect of each systematic uncertainty on the best fit oscillation values, a Monte Carlo test is run with shifted fake data sets. Each systematic is considered independently and the total uncertainty is the sum in quadrature of each individual uncertainty according to

$$
\sigma_{t o t}=\sqrt{\sum_{i}\left[\frac{1}{2}\left(\left|\sigma_{i+}\right|+\left|\sigma_{i-}\right|\right)\right]^{2}+b^{2}}
$$

where $\sigma_{i \pm}$ corresponds to the $\pm 1 \sigma$ shift, and $b$ is the shift in the oscillation parameter due to a combination of the F/N extrapolation and fitting technique. The full results of the uncertainty on the value of $\theta_{23}$ and $\Delta \mathrm{m}^{2}$ are shown in Table 6.2 ,

An example of a shift in absolute hadronic energy of the Monte Carlo is shown in Fig. 6.5, the remaining systematic plots can be found in Appendix B. The $+1 \sigma$ change denotes that the reconstructed energy of the event should be higher than the current value in both the Near and Far Detector, while the true energy of the event stays the same.

Because the first two energy bins coincide with the rising edge of the NC spectrum, more events will be shifted from energy bin $X$ into the next higher energy bin, $\mathrm{X}+1$, than will migrate from bin $\mathrm{X}-1$ to $\mathrm{X}$. On the rising edge the nominal spectrum will have more events than the $+1 \sigma$ shifted spectrum, which drives the nominal/shifted ratio above 1 . Because the peak of the spectrum occurs at $\sim 2 \mathrm{GeV}$, one anticipates that events congregate at the low energy edge of the bin versus the high, and the nominal/shifted ratio would be below 1 . Instead of a ratio crossover at $2 \mathrm{GeV}$, Fig. 6.5 shows the cross-over at $4 \mathrm{GeV}$. The reason lies in the $\mathrm{CC}$ background.

Both the nominal and shifted spectrum are the combination of the NC events 

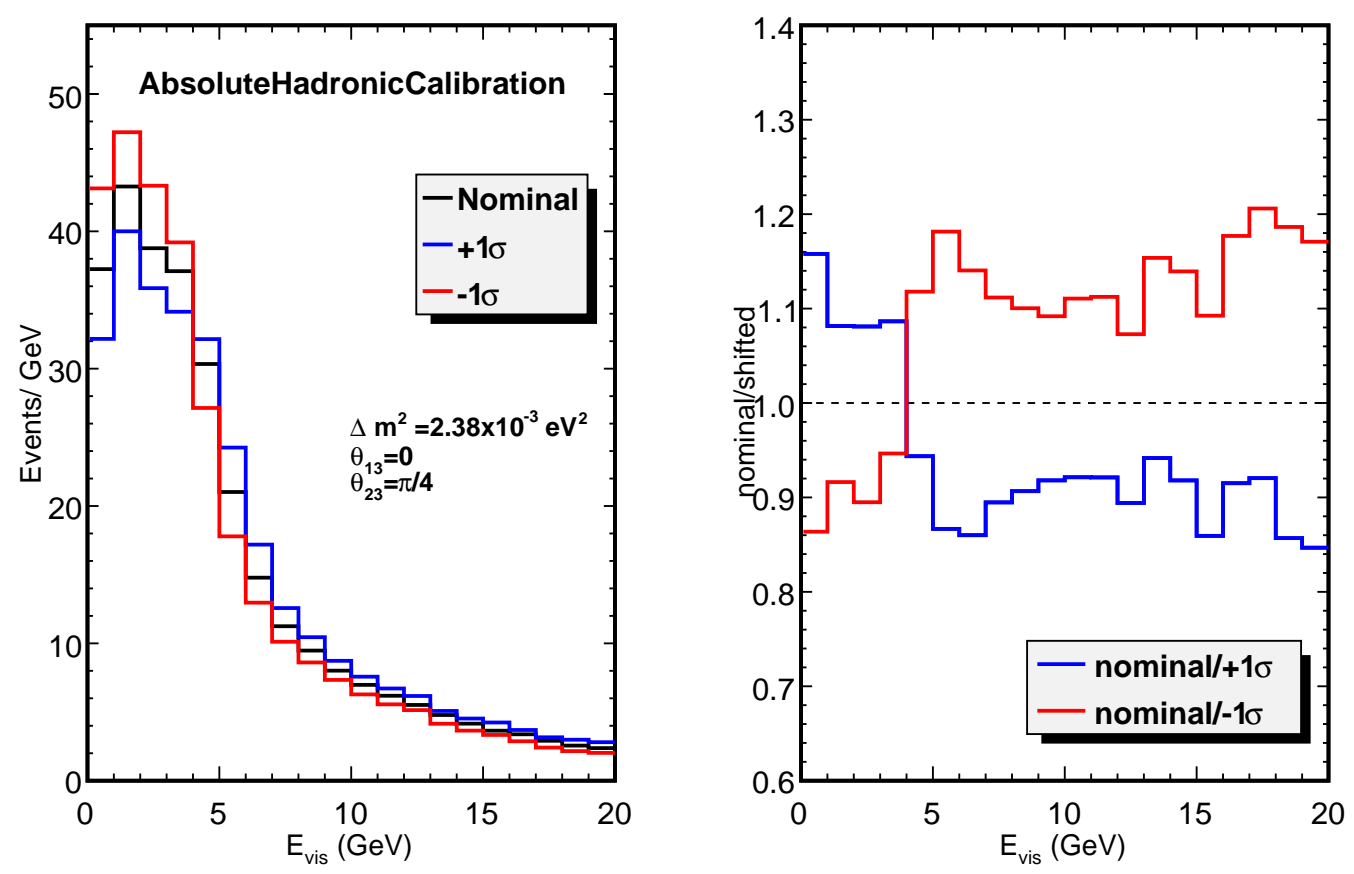

Figure 6.5: Shift in the F/N predicted spectrum when the absolute hadronic energy is shifted by $11 \%$. The plot on the left shows the nominal MC in black, $+1 \sigma$ shifted $\mathrm{MC}$ in red and $-1 \sigma$ shifted MC in black. The plot on the right shows the ratio of the nominal to the shifted predicted Far Detector energy spectrum.

\begin{tabular}{|c|c|c|c|c|c|c|}
\hline \multirow{2}{*}{ Systematic } & \multicolumn{3}{|c|}{$\Delta \mathrm{m}_{31}^{2}\left(10^{-3} \mathrm{eV}^{2}\right)$} & \multicolumn{3}{c|}{$\theta_{23}$} \\
\cline { 2 - 7 } & $+1 \sigma$ & $-1 \sigma$ & $(\text { Avg. Shift })^{2}$ & $+1 \sigma$ & $-1 \sigma$ & (Avg. Shift) $^{2}$ \\
\hline Nominal & - & - & $3.1810 \mathrm{e}-05$ & - & - & $7.35296 \mathrm{e}-08$ \\
Normalization & 2.4789 & 2.2915 & 0.0087797 & 0.78728 & 0.77346 & $4.7769 \mathrm{e}-05$ \\
AbsoluteHadronic & 2.3166 & 2.4484 & 0.0043428 & 0.78430 & 0.76655 & $9.9484 \mathrm{e}-05$ \\
RelativeHadronic & 2.3683 & 2.415 & 0.0005452 & 0.73435 & 0.78540 & $6.5156 \mathrm{e}-04$ \\
CCBackground & 2.3772 & 2.39 & $4.096 \mathrm{e}-05$ & 0.76891 & 0.78618 & $7.4641 \mathrm{e}-05$ \\
LowCompleteness & 2.3828 & 2.3825 & $7.0225 \mathrm{e}-06$ & 0.78508 & 0.78508 & $9.8699 \mathrm{e}-08$ \\
CCMA & 2.3691 & 2.3913 & 0.0001232 & 0.78508 & 0.77550 & $2.60627 \mathrm{e}-05$ \\
NCFarCleanNoise & 2.382 & 2.3833 & $7.0225 \mathrm{e}-06$ & 0.78524 & 0.78477 & $1.54185 \mathrm{e}-07$ \\
NCNearClean & 2.3849 & 2.381 & $8.7025 \mathrm{e}-06$ & 0.77629 & 0.78540 & $2.07495 \mathrm{e}-05$ \\
SKZPTV & 2.378 & 2.3869 & $1.9803 \mathrm{e}-05$ & 0.78493 & 0.78524 & $9.86986 \mathrm{e}-08$ \\
\hline
\end{tabular}

\begin{tabular}{|l|l|l|l|}
\hline Combined Syst. & 0.117925 & Combined Syst. & 0.0303429 \\
\hline
\end{tabular}

Table 6.2: Shifts in the oscillation parameters from the systematics in the 3Flavor model. The input values are $\Delta \mathrm{m}_{31}^{2}=2.38 \times 10^{-3} \mathrm{eV}^{2}, \theta_{23}=\pi / 4$ and $\theta_{13}=0$. The $\pm 1 \sigma$ columns are the results from the systematic shifts and the combined systematic uncertainty is the sum in quadrature of the individual shifts. 
as well as the background CC events, and it is the lack of effect that the uncertainty has on the $\mathrm{CC}$ events that helps push the turnover to $4 \mathrm{GeV}$. In this vein the nominal/shifted Monte Carlo can be thought of in terms of the $\mathrm{F} / \mathrm{N}$ extrapolation below:

$$
\begin{aligned}
\text { nominal/shifted } & =N D_{\text {data }}\left(\frac{F D_{M C}}{N D_{M C}}\right)_{\text {nominal }} / N D_{\text {data }}\left(\frac{F D_{M C}}{N D_{M C}}\right)_{\text {shifted }} \\
& =\left(\frac{F D_{M C}}{N D_{M C}}\right)_{\text {nominal }} /\left(\frac{F D_{M C}}{N D_{M C}}\right)_{\text {shifted }}
\end{aligned}
$$

At the Far Detector the $v_{\mu} \mathrm{CC}$ background is significantly diminished compared to the Near because of oscillation; each FD energy bin $<5 \mathrm{GeV}$ has a higher purity of NC events than the corresponding Near Detector bin 1 . The higher purity of NC events causes the $10 \%$ hadronic shift to impact the FD more than the ND over the energy range $0-5 \mathrm{GeV}$, and helps drive the turnover to $4 \mathrm{GeV}$.

The Absolute Hadronic systematic uncertainty creates a sizeable shift from the nominal spectrum at the $1 \sigma$ level, but a shift that the F/N method can correct. What is more important than the degree of the systematic shift, is the uncertainty in the number of events in each energy bin that the uncertainty introduces. The uncertainty in the number of events is calculated over two energy regions, $0-3 \mathrm{GeV}$ and 0-5 GeV, which correspond to the energies for which MINOS is most sensitive to neutrino oscillations. The total uncertainty is the average of all the $+1 \sigma$ shifts and the $-1 \sigma$ shifts added in quadrature. Table 6.3 shows the uncertainty in the number of events, as well as the relative uncertainty, from all the systematic uncertainties. The systematics that cause a relative difference between detectors, such as the Normalization and RelativeHadronicCalibration, cause the largest uncertaintity in the number of events.

\footnotetext{
${ }^{1} 5 \mathrm{GeV}$ is approximately where the $v_{\mu}$ survival exceeds $90 \%$.
} 


\begin{tabular}{|c|c|c|c|c|}
\hline \multirow{2}{*}{ Systematic $\left(\theta_{13}=0\right)$} & \multicolumn{2}{|c|}{$0-3 \mathrm{GeV}$} & \multicolumn{2}{c|}{$0-5 \mathrm{GeV}$} \\
\cline { 2 - 5 } & Events & Relative & Events & Relative \\
\hline Normalization & 4.41768 & 0.0369753 & 6.21636 & 0.0520299 \\
AbsoluteHadronicCalibration & 0.374104 & 0.00313118 & 0.857914 & 0.0071806 \\
RelativeHadronicCalibration & 3.35053 & 0.0280434 & 3.22549 & 0.0269968 \\
CCBackground & 3.0839 & 0.0258117 & 4.19841 & 0.03514 \\
LowCompleteness & 0.23814 & 0.00199319 & 0.261986 & 0.00219278 \\
CCMA & 1.11502 & 0.00933256 & 0.676464 & 0.00566189 \\
NCFarCleanNoise & 1.04817 & 0.00877301 & 1.03454 & 0.00865891 \\
NCNearClean & 2.62104 & 0.0219376 & 2.65685 & 0.0222374 \\
SKZPTV & 0.146887 & 0.00122942 & 0.240366 & 0.00201182 \\
\hline TOTAL & 7.04858 & 0.0589954 & 8.72483 & 0.0730254 \\
\hline \hline \multirow{2}{|c|}{$0-3 \mathrm{GeV}$} & & $0-5 \mathrm{GeV}$ \\
Systematic $\left(\theta_{13}=0.21\right)$ & Events & Relative & Events & Relative \\
\cline { 2 - 5 } & 3.66662 & 0.030689 & 4.91317 & 0.0411224 \\
Normalization & 0.611263 & 0.00511617 & 0.530388 & 0.00443926 \\
AbsoluteHadronicCalibration & 3.8026 & 0.0318271 & 3.44259 & 0.0288139 \\
RelativeHadronicCalibration & 2.6205 & 0.0219332 & 3.27785 & 0.027435 \\
CCBackground & 0.256357 & 0.00214567 & 0.248619 & 0.0020809 \\
LowCompleteness & 1.09909 & 0.00919923 & 1.21111 & 0.0101368 \\
CCMA & 1.03812 & 0.00868888 & 1.02704 & 0.00859619 \\
NCFarCleanNoise & 2.65181 & 0.0221952 & 2.6037 & 0.0217926 \\
NCNearClean & 0.253742 & 0.00212378 & 0.243444 & 0.00203759 \\
SKZPTV & 6.67775 & 0.0558917 & 7.51254 & 0.0628787 \\
\hline TOTAL & \multicolumn{2}{|c}{} \\
\hline
\end{tabular}

Table 6.3: The uncertaintity on the number of events in the Far Detector neutralcurrent spectrum for each systematic. The values are calculated for both $\theta_{13}=0$ and $\theta_{13}=0.21$, with an exposure of $2.5 \times 10^{20}$ POT. The main contributions are from systematics that change the relative number of events between the Near and Far Detector, specifically the Normalization. 


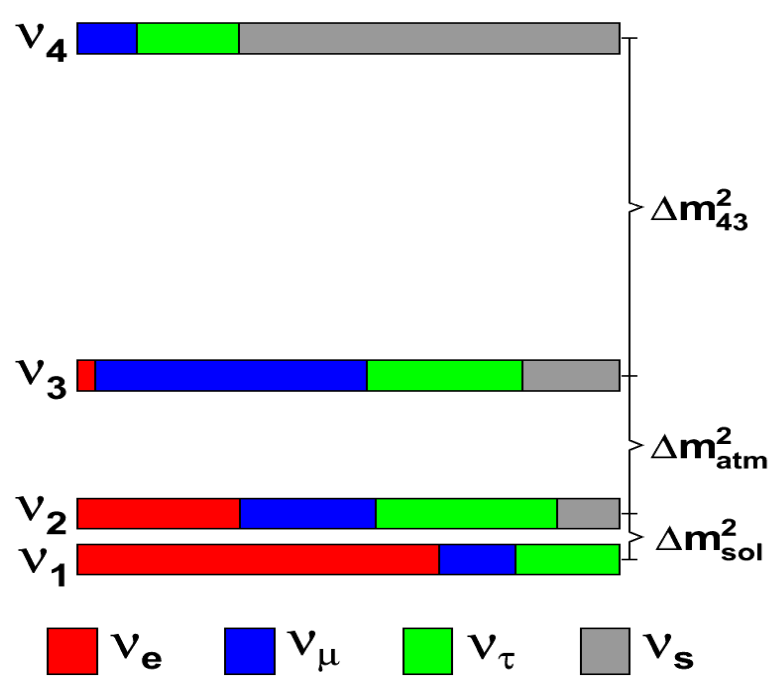

Figure 6.6: 4Flavor Parke model of neutrino oscillation. This is the standard 3Flavor model with the addition of a $4^{\text {th }}$ mass eigenstate that allows for transition to a sterile neutrino. This particular figure is made using the following values: $\left|U_{e 3}\right|^{2}=0.02$ which is $1 / 2$ the CHOOZ limit, $\left|U_{\mu 3}\right|^{2}=0.5$ which is the best fit value from both MINOS and Super-K, $\left|U_{s 3}\right|^{2}=0.18$ and $\left|U_{\tau 3}\right|^{2}=1-\left|U_{e 3}\right|^{2}-$ $\left|U_{\mu 3}\right|^{2}-\left|U_{s 3}\right|^{2}$.

\subsection{Flavor Model}

The 4Flavor model is an extension of the 3Flavor model by addition of a $4^{\text {th }}$ mass eigenstate, which allows not only the normal transitions of the three known neutrino flavors, but also the transition to a sterile neutrino. By including a $v_{\mu} \rightarrow v_{s}$ transition, MINOS can use neutral-current events to measure the PMNS-like matrix elements associated with a sterile neutrino, namely the amount of sterile flavor in the $3^{r d}$ mass eigenstate, $\left|U_{s 3}\right|^{2}$. A measurement of zero does not necessarily exclude the existence of a sterile neutrino, but does exclude it in the unique parameter space available to MINOS.

It is worth noting that while the $v_{1}$ eigenstate has a well measured admixture of $v_{e}, v_{\mu}$ and $v_{\tau}$ flavor, the third mass eigenstate has only two well measured mixings; CHOOZ has limited $\left|U_{e 3}\right|^{2} \leq 0.05$, while atmospheric and accelerator experiments, most notably Super-K, calculates the limit $0.45 \leq\left|U_{\mu 3}\right|^{2} \leq 0.50$. The remainder of the third mass eigenstate is currently assumed to be made up of $v_{\tau}$, but with 
the derth of $v_{\tau}$ appearance experiments, an actual measurement of $\left|U_{\tau 3}\right|^{2}$ remains conspicuously absent from the neutrino picture. Combined with small uncertainty on the amount of $v_{\mu}$ and $v_{\tau}$ flavor in the $2^{\text {nd }}$ mass eigenstate, a sprinkling of sterile flavor in the $3^{\text {rd }}$ mass eigenstate is not beyond the realm of possibility. Concurrent with sterile flavor in the $3^{\text {rd }}$ mass eigenstate, the $4^{\text {th }}$ mass eigenstate would contain small amounts of the $v_{\mu}$ and $v_{\tau}$ flavor. All told, the 4Flavor model, pictured in Fig. 6.6, is consistent with the major neutrino oscillation measurements, and exists as an experimentally viable means to probe the existence of a sterile neutrino.

The structure of the following 4Flavor MC section is similar to that of the 3Flavor, where $\mathrm{MC}$ tests validating that the $\mathrm{F} / \mathrm{N}$ extrapolation works with the new 4Flavor oscillation model and can return input values. Following the validation will be a study on the impact of the systematic uncertainties on the new oscillation parameters.

\subsubsection{F/N Validation using 4Flavor Model}

The F/N extrapolation is tested for the 4Flavor model using the same procedure outlined in Sec. 6.1.1. V1, V2 and systematics. Besides the addition of two more parameters, $\left|U_{\mu 4}\right|^{2}$ and $\left|U_{s 3}\right|^{2}$, the mass and flavor eigenstate relationship is cast in terms of the PMNS matrix elements, whereby $\left|U_{\mu 4}\right|^{2}$ refers to the fractional amount of $v_{\mu}$ flavor in the $4^{\text {th }}$ mass eigenstate and $\left|U_{s 3}\right|^{2}$ is the amount of $v_{s}$ in the $3^{r d}$ mass eigenstate. The plots in Fig. 6.7 show the V2 validation where the input values have a small amount, $10 \%$, of sterile mixing in the $4^{\text {th }}$ mass eigenstate.

The results in Table 6.4 from validating the $\mathrm{F} / \mathrm{N}$ extrapolation with the 4Flavor model using method V1 and V2, show good agreement between the input and best fit values. 

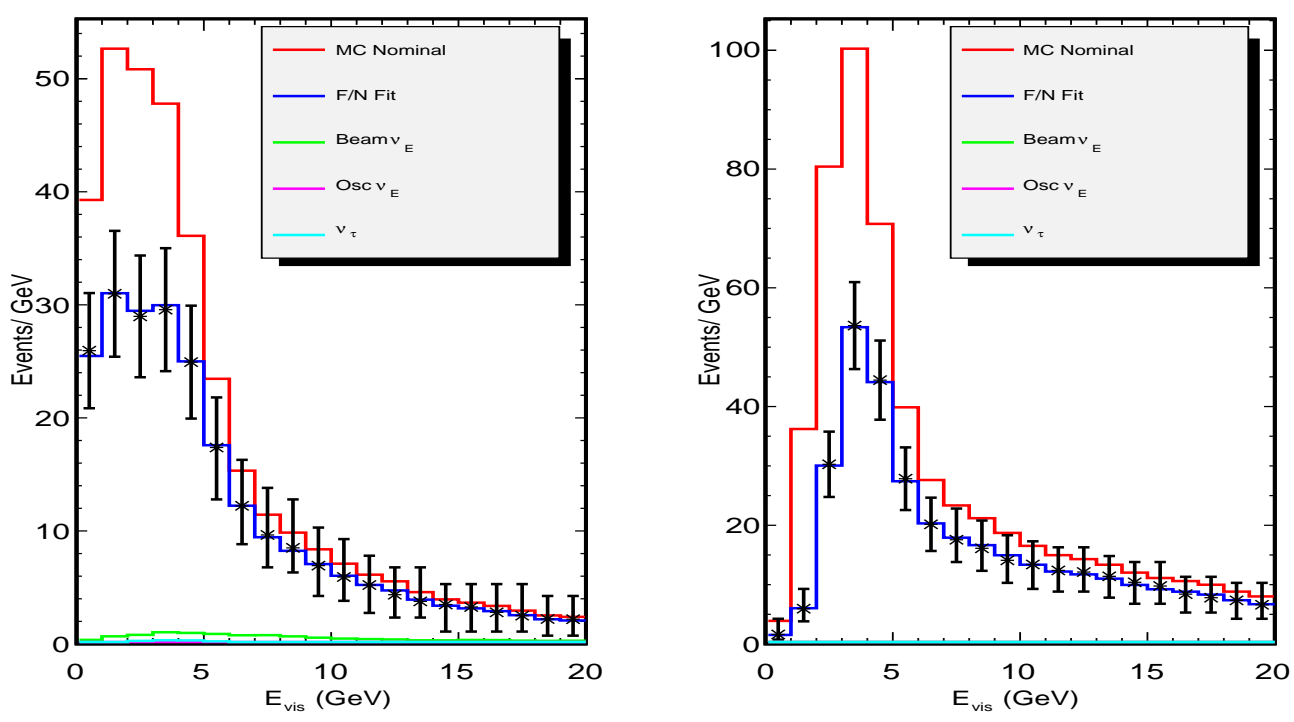

(a)
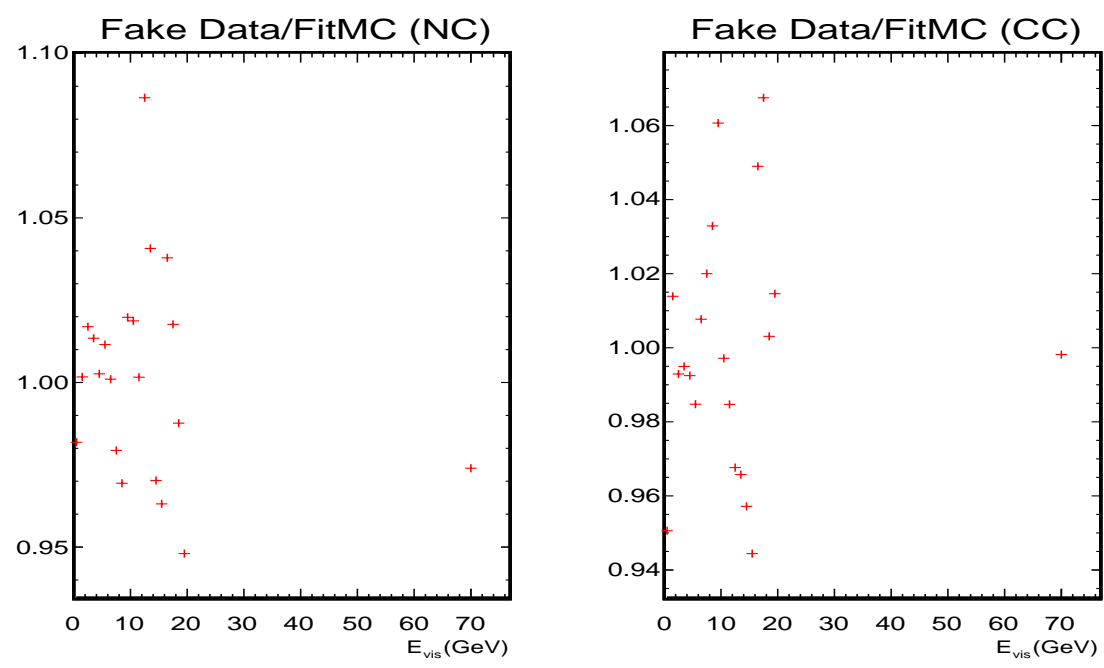

(b)

Figure 6.7: Using the V2 method of having two separate FD MC sets, figure (a) shows the predicted CC and NC energy spectrum results of a Monte Carlo test using the 4Flavor model without systematic uncertainty shifts, setting $\theta_{13}=0, \delta_{C P}=0$ and an exposure of $2.5 \times 10^{20}$ POT. The impact of the non-zero sterile neutrino contribution is largely seen through the peak region $(2-4 \mathrm{GeV})$ of the FD NC spectrum, which is reduced versus the 3Flavor model shown in Fig. 6.3(a). Figure (b) shows the bin by bin ratio of the data to the F/N Fit spectrum. 


\begin{tabular}{|c|c|c|c|c|c|c|c|c|}
\hline \multirow{2}{*}{ Method } & \multicolumn{5}{|c|}{ Input Value } & \multicolumn{5}{c|}{ Best Fit Value } \\
\cline { 2 - 9 } & $\Delta \mathrm{m}_{31}^{2}$ & $\left|U_{\mu 3}\right|^{2}$ & $\left|U_{\mu 4}\right|^{2}$ & $\left|U_{s 3}\right|^{2}$ & $\Delta \mathrm{m}_{31}^{2}$ & $\left|U_{\mu 3}\right|^{2}$ & $\left|U_{\mu 4}\right|^{2}$ & $\left|U_{s 3}\right|^{2}$ \\
\hline V1 & 2.43 & 0.45 & 0.1 & 0.07 & 2.4318 & 0.46523 & 0.09770 & 0.06973 \\
V2 & 2.43 & 0.45 & 0.1 & 0.07 & 2.4065 & 0.42478 & 0.09990 & 0.07533 \\
\hline
\end{tabular}

Table 6.4: Results from the two different methods of validating the F/N method with the 4Flavor model. Both tests are done with $\theta_{13}=0, \delta_{C P}=0$ and an exposure of $2.5 \times 10^{20}$ POT. The units for the $\Delta \mathrm{m}^{2}$ values are in $10^{-3} \mathrm{eV}^{2}$.

\subsubsection{Systematic Uncertainty Shifts}

Evaluating the effect of the systematic uncertainties for the 4Flavor model follows the same procedure as the 3Flavor model in Sec. 6.1.2. The change in oscillation parameters $\Delta \mathrm{m}_{31}^{2}, U_{\mu 3}, U_{s 3}$, and $U_{\mu 4}$ for a $\pm 1 \sigma$ shift is calculated for each systematic and shown in Table 6.5. The addition of two extra fit parameters versus the 3Flavor model contributes to the decrease of the impact of the uncertainties from Table 6.2

\subsection{Data Results}

\subsubsection{Flavor}

The test of sterile neutrino existence using the 3Flavor model is based on the level of agreement between data and prediction in the energy range $<5 \mathrm{GeV}$. This region corresponds to the highest MINOS sensitivity to a neutral-current rate deficit caused by oscillation to a sterile neutrino. However, this energy region also has the drawback that $v_{e}$ appearance becomes a significant background. The impact of this $v_{e}$ background will be discussed later, while Fig. 6.8 compares data and prediction for the case of $\theta_{13}=0$. The charged-current plot illustrates that the $\mathrm{F} / \mathrm{N}$ prediction closely matches the data, where the fit value of $\Delta \mathrm{m}_{31}^{2}=2.60 \times 10^{-3} \mathrm{eV}$ is used to calculate the oscillation of the $\mathrm{CC}$ background in the NC events sample. The effect of oscillation in the $\mathrm{CC}$ background appears in the $\mathrm{NC}$ plot as the difference between the Nominal non-oscillated MC and the F/N Fit. The number of data NC events in 


\section{完 \\ क त्ञ \\ 을 \\ 要}

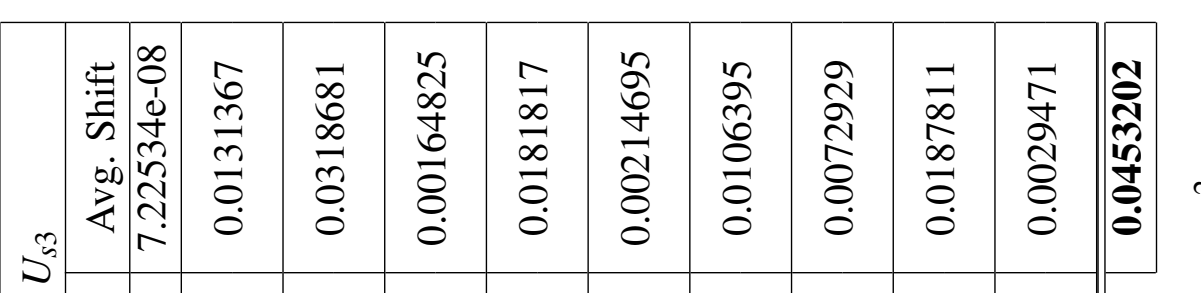

i.

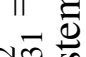

तले क्ष

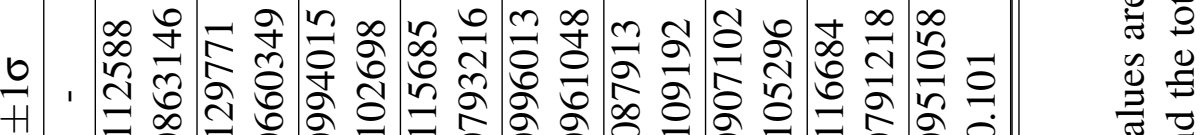

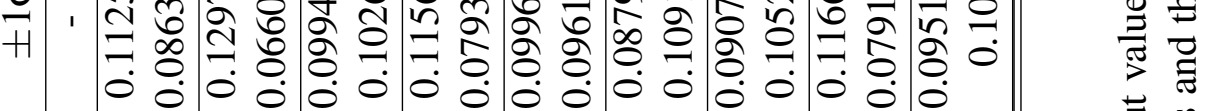

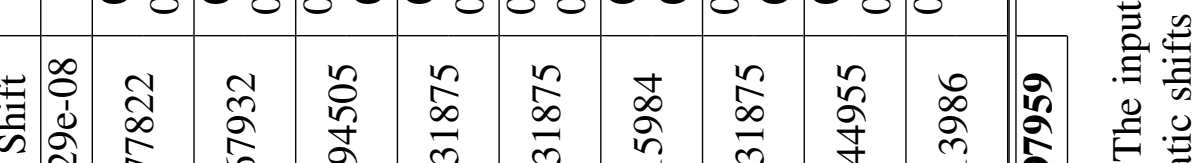

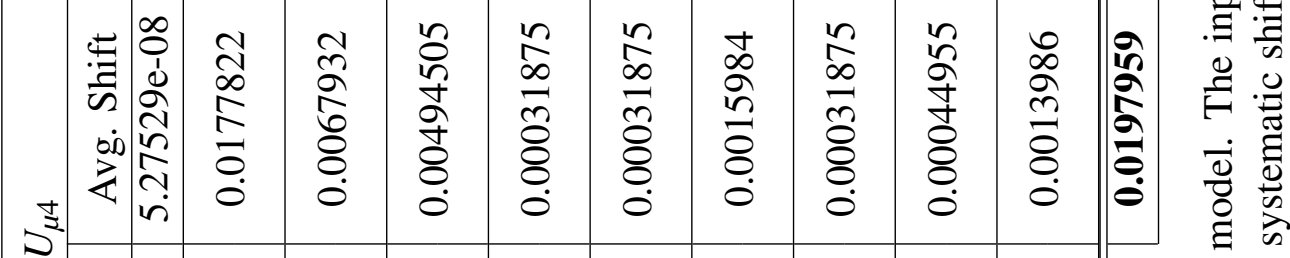

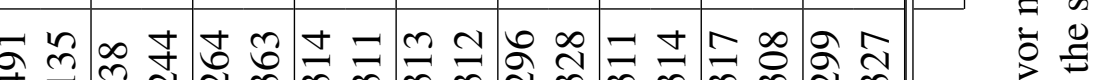

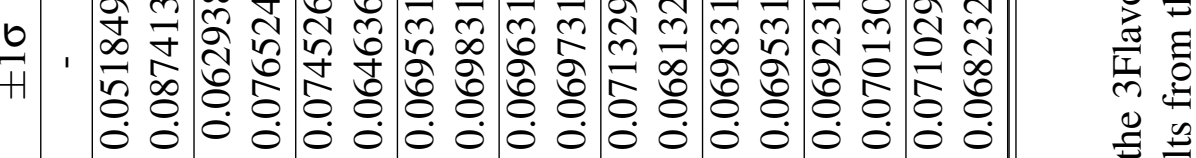

\begin{tabular}{|c|c|c|c|c|c|c|c|c|}
\hline 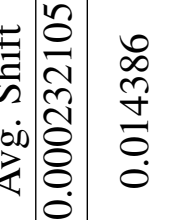 & $\begin{array}{l}n \\
\tilde{n} \\
\infty \\
\stackrel{+}{+} \\
0 \\
0 \\
0\end{array}$ & $\begin{array}{l}\tilde{O} \\
0 \\
\infty \\
0 \\
0 \\
0\end{array}$ & \begin{tabular}{l}
$n$ \\
2 \\
$\infty$ \\
\multirow{8}{+}{} \\
0 \\
0
\end{tabular} & $\begin{array}{l}n \\
\frac{n}{n} \\
\frac{n}{n} \\
0 \\
0\end{array}$ & $\begin{array}{l}2 \\
0 \\
n \\
n \\
0 \\
0 \\
0\end{array}$ & $\begin{array}{l}\infty \\
\infty \\
\stackrel{\infty}{+} \\
\stackrel{0}{0} \\
\dot{0}\end{array}$ & $\begin{array}{l}n \\
2 \\
\infty \\
o \\
o \\
0 \\
0\end{array}$ & $\begin{array}{l}n \\
2 \\
\infty \\
\infty \\
+ \\
0 \\
0\end{array}$ \\
\hline
\end{tabular}

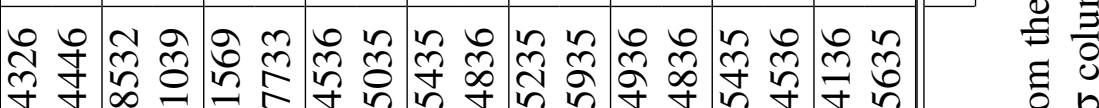

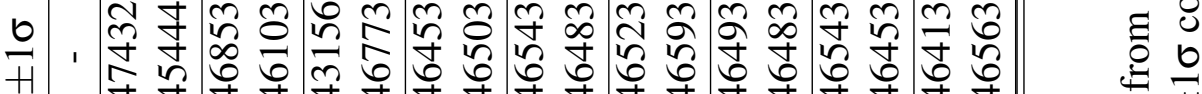

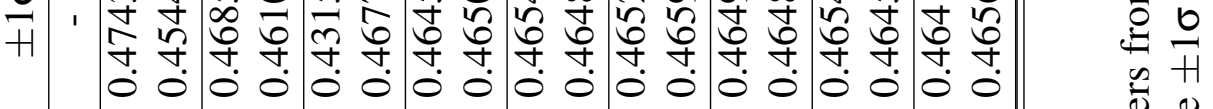

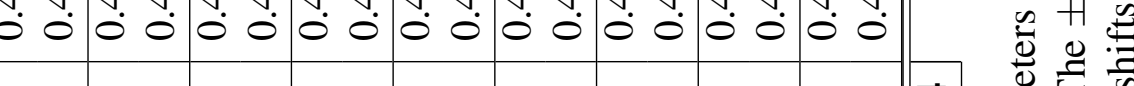

\begin{tabular}{|c|c|c|c|c|c|c|c|c|c|c|c|}
\hline & 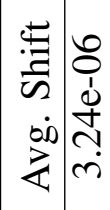 & 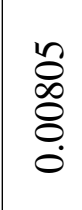 & $\begin{array}{l}\text { ठे. } \\
\text { }\end{array}$ & 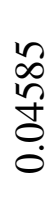 & 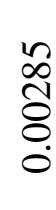 & $\begin{array}{l}\frac{n}{\delta} \\
\frac{\delta}{0} \\
0\end{array}$ & 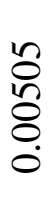 & $\begin{array}{l}\frac{n}{\widetilde{\delta}} \\
\stackrel{0}{0}\end{array}$ & 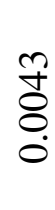 & 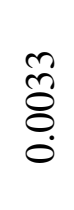 & 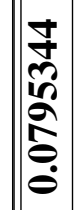 \\
\hline
\end{tabular}

匀

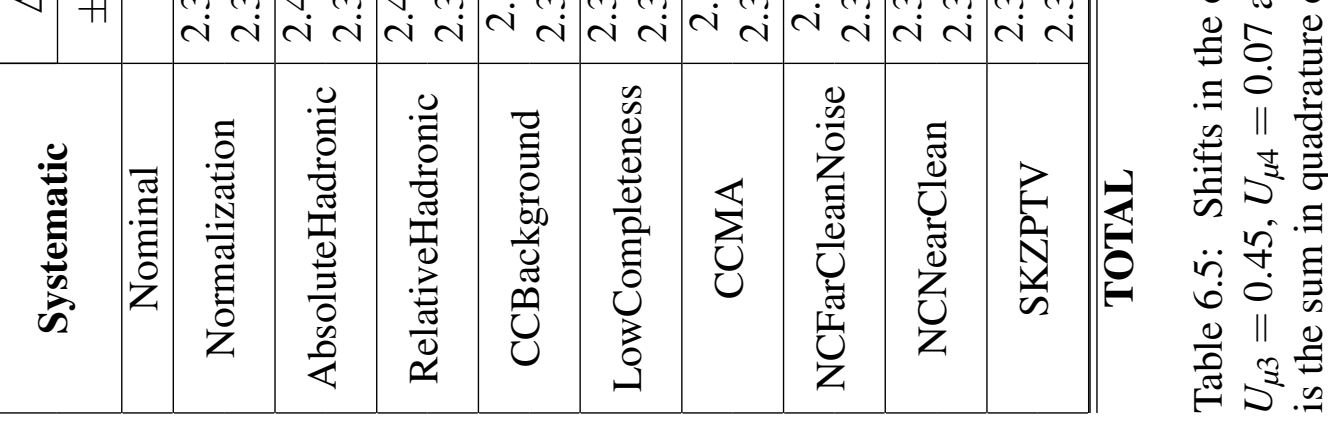



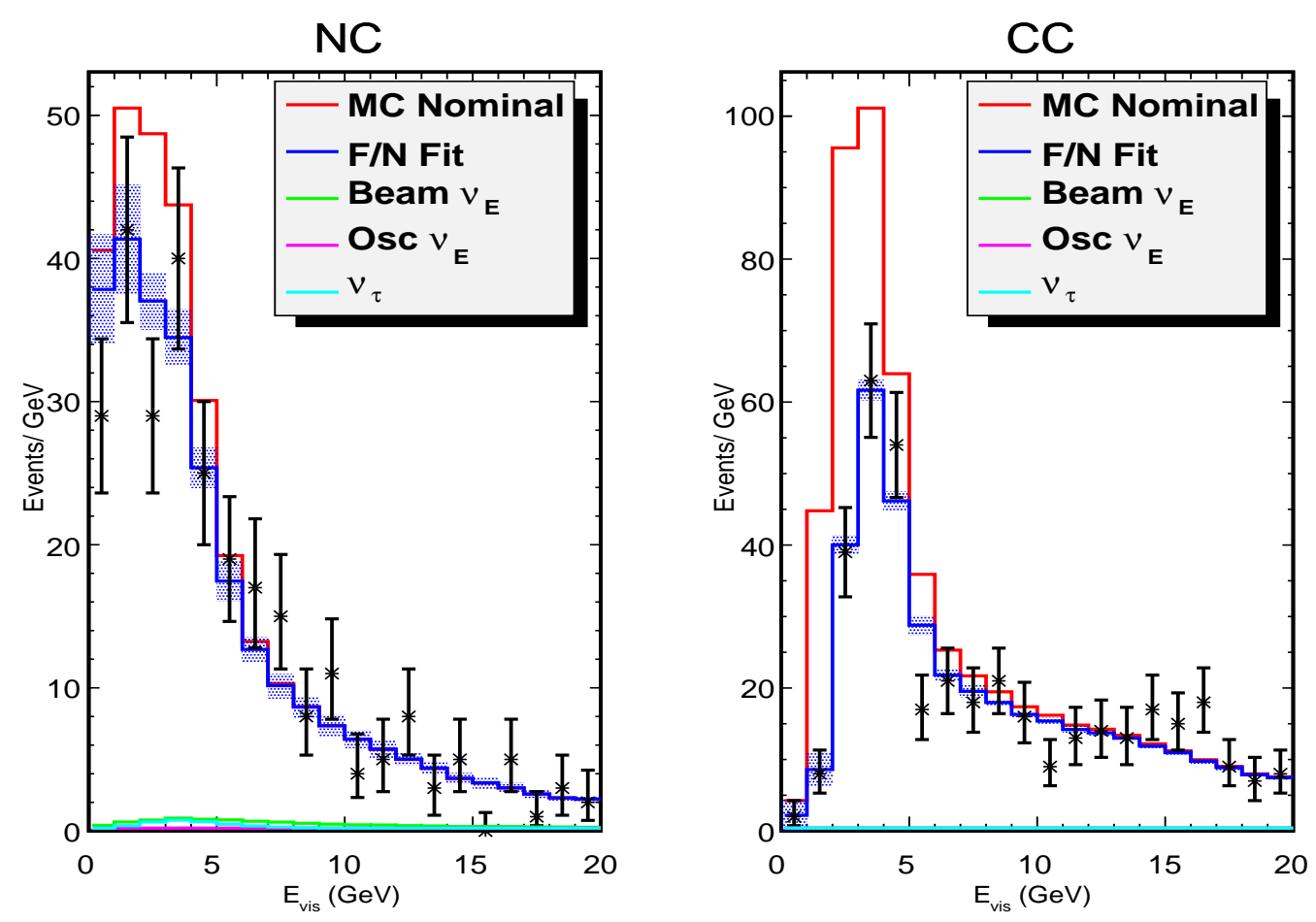

Figure 6.8: 3Flavor neutral-current and charged-current energy spectrum for the combined RunI and RunII data set with a Far Detector exposure of $2.46 \times 10^{20}$ POT. The $\mathrm{F} / \mathrm{N}$ prediction is for no $\mathrm{v}_{e}$ appearance or $\theta_{13}=0$. The unoscillated Monte Carlo is in red, $\mathrm{F} / \mathrm{N}$ prediction is in blue and the data points are in black. The blue error band is the systematic uncertainty in each bin of energy. The plot on the right is the charged-current event energy spectrum, selected using the NC Analysis PID from Sec. 5.4, which is fit to ascertain the value of $\Delta \mathrm{m}_{31}^{2}$ and used to oscillate the $\mathrm{CC}$ background in the NC selected sample.

the $0-1$ and 2-3 GeV energy bins deviates slightly from the F/N prediction, but the rest of the data closely matches the prediction and are within the error bars. The level of agreement over the two aforementioned energy ranges combined with the two extrema of $v_{e}$ appearance are explained in further detail below, with the event rate and $\chi^{2}$ values for the fits shown in Table 6.6. For a further analysis of the CC spectrum see [36].

Maximally enhancing $v_{e}$ appearance, by setting $\theta_{13}=0.21$ and $\delta_{C P}=3 \pi / 2$, introduces $22 v_{e}$ charged-current events that are selected as $\mathrm{NC}$ in the $0-5 \mathrm{GeV}$ energy range. The background increases the lower energy disagreement between 

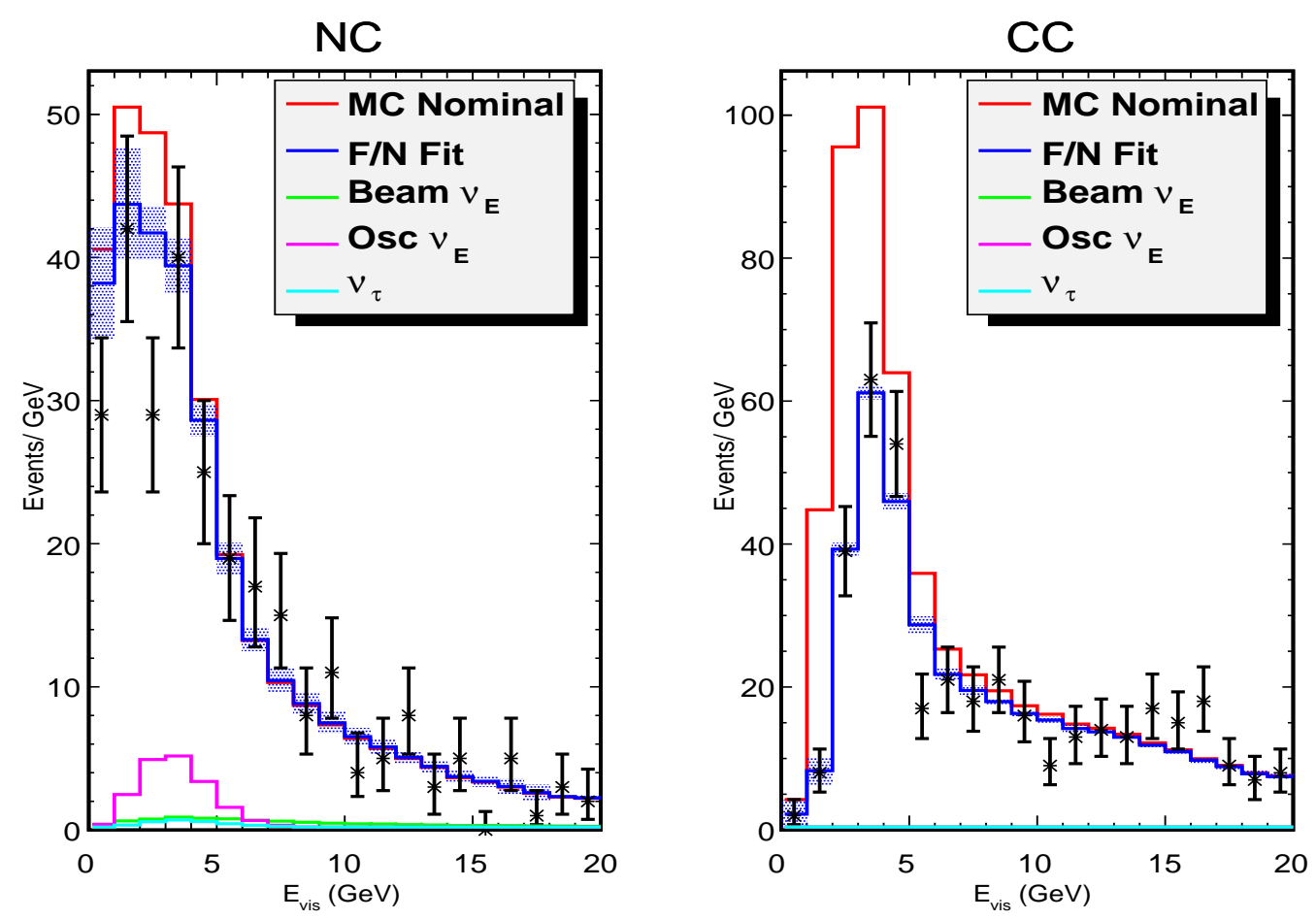

Figure 6.9: 3Flavor neutral-current and charged-current energy spectrum for the combined RunI and RunII data set with a Far Detector exposure of $2.46 \times 10^{20}$ POT. The $v_{\mu} \rightarrow v_{e}$ transition is maximally enhanced by setting $\theta_{13}=0.21$ and $\delta_{C P}=$ $3 \pi / 2$. The unoscillated Monte Carlo is in red, F/N prediction is in blue and the data points are in black.

the NC F/N prediction and the data, shown in Fig. 6.9. Besides directly changing the NC spectrum, the $v_{e}$ background has an indirect influence by affecting the best fit value of $\Delta \mathrm{m}_{31}^{2}$ value. As a direct consequence, to create better agreement between data and MC the first and third energy bin pull $\Delta \mathrm{m}_{31}^{2}$ to a lower value, $2.52 \times 10^{-3} \mathrm{eV}$, than seen in the $\theta_{13}=0$ prediction in order create more CC background disappearance to compensate for the $v_{e}$ appearance.

The level of agreement between prediction and data is quantatively assessed in terms of a significance statistic defined as

$$
\sigma_{s i g}=\frac{N_{\text {data }}-N_{M C}}{\sqrt{N_{M C}+\sigma_{s y s t}^{2}}}
$$




\begin{tabular}{|c|c|c|c|c|c|}
\hline $\begin{array}{c}\text { Energy } \\
(\mathrm{GeV})\end{array}$ & Data & $\begin{array}{c}\text { MC } \\
\left(\theta_{13}=0\right)\end{array}$ & Significance & $\begin{array}{c}\text { MC } \\
\left(\theta_{13}=0.21\right)\end{array}$ & Significance \\
\hline $0-3$ & 100 & $116.21 \pm 7.0486$ & 1.4600 & $123.626 \pm 6.6777$ & 2.0697 \\
$0-5$ & 165 & $176.058 \pm 8.7248$ & 0.813456 & $191.676 \pm 7.5125$ & 1.8901 \\
\hline
\end{tabular}

Table 6.6: Number of events and level of agreement between data and F/N neutralcurrent prediction for the two MINOS sensitive energy regions.

where $N_{\text {data }}$ and $N_{M C}$ are the respective amount of neutral-current events. The results of the significance statistic are found in Table 6.6. The case where $\theta_{13}=0$ shows good agreement between data and prediction, while the prediction at the CHOOZ limit shows $\mathrm{a} \sim 2 \sigma$ deviation across both energy regions.

The two predictions represent the upper and lower bounds of agreement for the combined RunI+RunII data set, where an upcoming measurement of $\theta_{13}[85$ 86, 87] will refine the level of agreement. In terms of a sterile neutrino signature the data/MC comparison using the 3Flavor model is consistent with no sterile neutrinos, and with further statistics and a definite $\theta_{13}$ measurement, future MINOS results measuring the rate of $\mathrm{NC}$ events remain will be improved.

\subsubsection{Flavor}

The NC analysis using the 4Flavor model tests the existence of a sterile neutrino by measuring the amount of sterile flavor in the third mass eigenstate. The $\left|U_{s 3}\right|^{2}$ value is extracted after a $\chi^{2}$ minimization between data and $\mathrm{F} / \mathrm{N}$ prediction, which produces the fit spectrum shown in Fig. 6.10. In comparison to the fit spectrum using the 3Flavor model from Fig. 6.9, there is better agreement with the Monte Carlo, where both include $\mathrm{v}_{e}$ appearance at the CHOOZ limit. But, using the 4Flavor model introduces a new facet versus the basic Data/MC agreement because the model can be used in a fitting routine to return oscillation parameters.

Similar to the case using the 3Flavor model, the 4Flavor model uses the extrema of $v_{e}$ appearance to test the sterile neutrino hypothesis: no appearance and appear- 

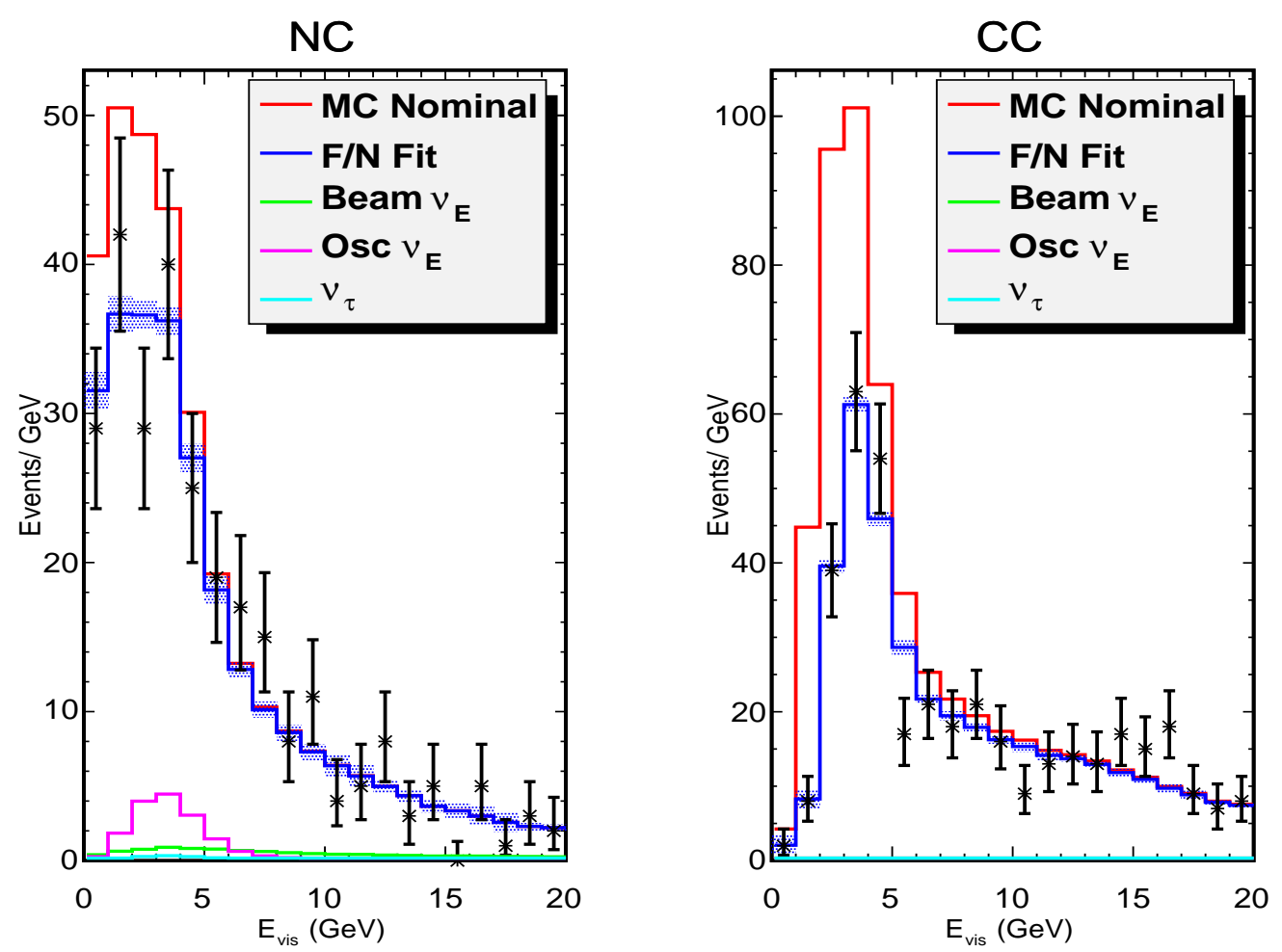

Figure 6.10: 4Flavor neutral-current and charged-current energy spectrum for the combined RunI and RunII data set with a Far Detector exposure of $2.46 \times 10^{20}$ POT. The spectra represent $v_{e}$ appearance at the $\mathrm{CHOOZ}$ limit using the best fit points $\left|U_{\mu 3}\right|^{2}=0.52$ and $\left|U_{s 3}\right|^{2}=0.19$, where the data are represented as the black points.

ance at the $\mathrm{CHOOZ}$ limit. While the difference between the two extrema of $v_{e}$ appearance does not largely affect the value $\left|U_{\mu 3}\right|^{2}$, the $\left|U_{s 3}\right|^{2}$ parameter does possess sensitivity. $\left|U_{\mu 3}\right|^{2}$ is unaffected because it is derived from $C C v_{\mu}$ events, which have a track-like topology that is easily separated from small shower-like events created by CC $v_{e}$ events. The $\left|U_{s 3}\right|^{2}$ parameter is sensitive because the added CC $v_{e}$ background appears in the peak of the NC energy spectrum, 0-5 GeV, which is the same region where a larger value of $\left|U_{s 3}\right|^{2}$ will result in events oscillating to sterile neutrinos.

The confidence limits of the oscillation parameter of main importance, $\left|U_{s 3}\right|^{2}$, are shown in Fig. 6.11 along with the $68 \%$ and $90 \%$ contours of $\left|U_{\mu 3}\right|^{2}$ vs. $\left|U_{s 3}\right|^{2}$. The non-zero $\left|U_{s 3}\right|^{2}$ best fit values for both extrema of $v_{e}$ appearance is the result 
of the NC data, shown in the left energy spectrum plot in 6.10 , being below the best fit expectation. In order to create better agreement between MC and data the fitting procedure removes neutrino events in the peak by having them oscillate to noninteracting sterile neutrinos. The minimal impact on the $\left|U_{\mu 3}\right|^{2}$ parameter from both the $\left|U_{s 3}\right|^{2}$ non-zero best fit values and $v_{e}$ appearance is shown by the tight overlap of the $\chi^{2}$ projections in the top left plot of Fig. 6.11

The test of sterile neutrino existence using the 4Flavor model is the amount of sterile flavor in the $3^{r d}$ mass eigenstate, $\left|U_{s 3}\right|^{2}$. The $\left|U_{\mu 3}\right|^{2}$ versus $\left|U_{s 3}\right|^{2}$ contours in Fig. 6.11 show that for both $v_{e}$ appearance extrema the $90 \%$ confidence interval allows $\left|U_{s 3}\right|^{2}=0$ over a wide range of $\left|U_{\mu 3}\right|^{2}$, which is consistent with no sterile neutrino appearance. From the $\chi^{2}$ projections $\left|U_{s 3}\right|^{2}<0.38(0.45)$ at the $90 \%$ C.L. for $\theta_{13}=0(0.21)$, where the limits are calculated using the associated best fit values of $\left|U_{\mu 3}\right|^{2}$. Both the contours and $\chi^{2}$ projections indicate that the 4 Flavor model fits the data as well as the standard 3Flavor model. 

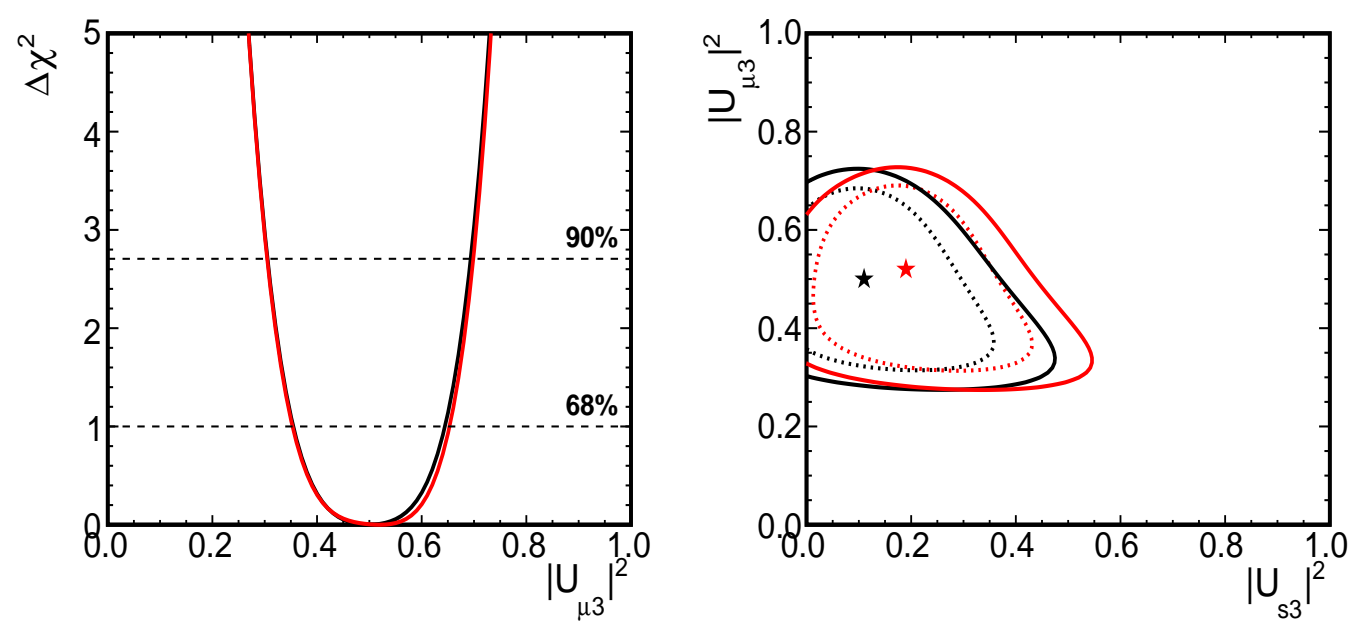

- No $v_{\mathrm{e}}$ appearance

Best fit points: $\left|U_{\mu 3}\right|^{2}=0.50$

$\left|\mathrm{U}_{\mathrm{s} 3}\right|^{2}=0.11$

- $v_{\mathrm{e}}$ appearance at $\mathrm{CHOOZ}$ limit

Best fit points: $\left|\mathrm{U}_{\mu 3}\right|^{2}=0.52$

$$
\left|\mathrm{U}_{\mathrm{s} 3}\right|^{2}=0.19
$$

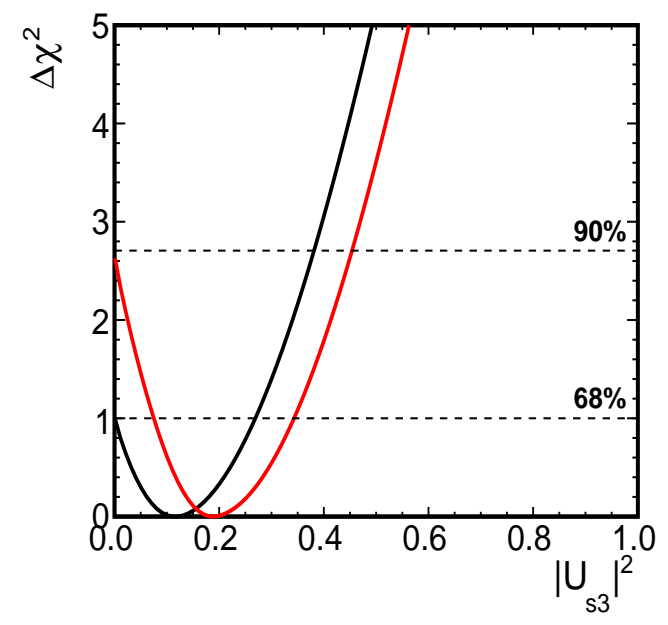

Figure 6.11: Contour plots, 1D projections and fit values of the 4Flavor model for the oscillation parameters $\left|U_{\mu 3}\right|^{2}$ and $\left|U_{s 3}\right|^{2}$. The 1D projections are computed by fixing the corresponding oscillation parameters to their best fit value, i.e. the $\left|U_{\mu 3}\right|^{2}$ plot uses $\left|U_{s 3}\right|^{2}=0.11$ or 0.19 . The vertical abscissa on the $1 \mathrm{D}$ plots are in units of $\Delta \chi^{2}$. The dashed lines in the contour plot represent the $68 \%$ confidence interval while the solid lines represents the $90 \%$ interval. 


\section{Chapter 7}

\section{Conclusion}

Since its discovery, the neutrino has been a source of intriguing physics. In order to understand the properties of neutrino oscillation the MINOS experiment has been constructed to use an accelerator neutrino beam, NuMI, over a long baseline to measure the parameters governing neutrino disappearance.

Two significant concerns for MINOS, and the accelerator neutrino physics community at large, are a calculation of the initial neutrino flux as well as the kinematics of the secondary pions and kaons from proton-target interactions. Because muons and neutrinos are created in a one-to-one relationship from the same parent hadrons, muons can be used to reduce the uncertainty on the flux and secondary hadron kinematics. To this end, a Geant4 NuMI Monte Carlo has been optimized to incorporate all the physical elements between the NuMI target and downstream muon monitors and make predictions of the signal at the muon monitors.

Besides the more conventional measurement of neutrino oscillation parameters, MINOS has the ability to look for sterile neutrinos. Because the $\mathrm{Z}^{0}$ couples only to the three light active flavors of the neutrino, a deficit of neutral-current events over a long baseline would be indicative of a neutrino transition to a non-active or sterile flavor. MINOS tests the existence of a sterile neutrino using the normal 3 flavor neutrino model as well as the 4Flavor Parke model. Using the 3 flavor model 
the level of agreement between the MINOS neutral-current data and prediction is $\sim 2 \sigma$ for the $0-5 \mathrm{GeV}$ and $0-3 \mathrm{GeV}$ range for $v_{e}$ appearance at the $\mathrm{CHOOZ}$ limit and $1.46 \sigma$ and $0.81 \sigma$ for the no $v_{e}$ appearance limit. The MINOS sensitivity to a sterile neutrino in the 4Flavor model comes in terms of the amount of sterile flavor in the third mass eigenstate, $\left|U_{s 3}\right|^{2}$. The NC analysis makes two separate measurements of $\left|U_{s 3}\right|^{2}$ to take into account the effect of $v_{e}$ appearance at the $\theta_{13}=$ 0.21 , the CHOOZ limit, and $\theta_{13}=0$. Joint fits to the observed neutral and chargedcurrent spectra using the 4Flavor oscillation model were consistent with no sterile neutrinos at the $90 \%$ confidence limit, and produced upper bounds of $\left|U_{s 3}\right|^{2}<0.38$ with no $v_{e}$ appearance and $\left|U_{s 3}\right|^{2}<0.45$ at the CHOOZ limit.

For sterile neutrinos, the near future neutrino oscillation experiments, such as NoVA and CNGS, will much the same parameter space to the MINOS experiment due to the similar beam kinematics as well as baselines. Without the advent of significantly higher flux accelerator beams or the development of a high intensity beam of $\tau$ neutrinos, looking for sterile neutrinos as a function of a neutral-current rate deficit will prove little better than MINOS measurements. What upcoming experiments, such as OPERA, may provide though, is a well measured value of $\left|U_{\tau 3}\right|^{2}$, which together with current precision on the values of $\left|U_{\mu 3}\right|^{2}$ and $\left|U_{e 3}\right|^{2}$, may provide a hint of sterile neutrinos if $\left|U_{e 3}\right|^{2}+\left|U_{\mu 3}\right|^{2}+\left|U_{\tau 3}\right|^{2} \neq 1$.

A promising area in the search for sterile neutrinos comes from shockwave development in core collapse supernovae[88]. The initial moments of a supernova from a stellar progenitor less than 20 solar masses contain the creation of a protoneutron star from the iron-nickel core of the original star. The stellar layers of oxygen, silicon, neon etc... fall onto the neutron star and rebound to generate an outward going shockwave. While the shockwave propagates away from the neutron star it encounters infalling matter which would ultimately stall the shockwave, resulting in the lack of an explosion. Ultimately it is the large amounts of neutri- 
nos, $\sim 10^{53} \mathrm{TeV}$, produced behind the shockfront which reenervate the outgoing shockwave through charged and neutral current interactions and allow the explosion. The existence of a sterile neutrino, and most importantly the possibility of a transition from active to sterile, would decrease the amount of energy deposited behind the shockwave and would extend the formation time between core collapse and explosion. While the physics community waits for near earth supernovae, the MINOS experiment will continue to collect data and examine the existence of a sterile neutrino for the upcoming few years. 


\section{Appendix A}

\section{Constraining the 3Flavor model}

\section{probabilities}

Including the matter effects in the 3Flavor Model is achieved through an expansion that while more accurate than the vacuum expression, introduces non-physical transition probabilities at low energies. To maintain physical probabilities all the transitions are constrained to within the physical range $0-1$. The difference between unconstrained and constrained probabilities for different values of $\theta_{13}$ and $\delta_{C P}$ in Table A.1 are shown in the following section.

\begin{tabular}{|c||c|c|c|c|c|c|}
\hline \multicolumn{1}{|c||}{ Set } & \multicolumn{6}{c|}{ Input Parameter } \\
\cline { 2 - 7 } & $\theta_{13}$ & $\theta_{23}$ & $\Delta m_{31}^{2}\left(10^{-3} \mathrm{eV}^{2}\right)$ & $\delta_{C P}$ & $\theta_{12}$ & $\Delta m_{12}^{2}\left(10^{-5} \mathrm{eV}^{2}\right)$ \\
\hline I & 0.21 & $\pi / 4$ & 2.38 & $3 \pi / 2$ & 0.61 & 7.59 \\
II & 0 & $\pi / 4$ & 2.38 & $3 \pi / 2$ & 0.61 & 7.59 \\
III & 0.21 & $\pi / 4$ & 2.38 & 0 & 0.61 & 7.59 \\
IV & 0 & $\pi / 4$ & 2.38 & 0 & 0.61 & 7.59 \\
\hline
\end{tabular}

Table A.1: Input parameters for the 3Flavor model. All values are best fit results from reactor, atmospheric and accelerator neutrino experiments, except for $\delta_{C P}$ which has no constraint between $0-2 \pi$. 


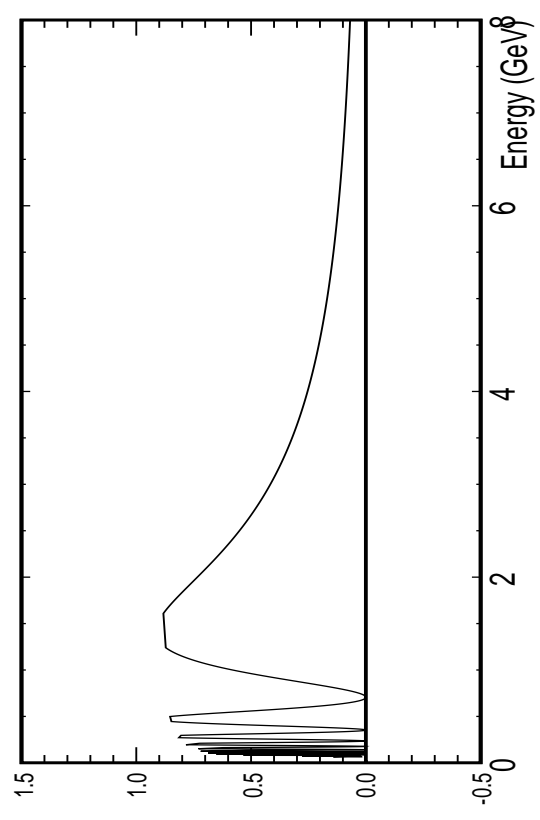

Uo!!! SUEג? ${ }^{2} \wedge$

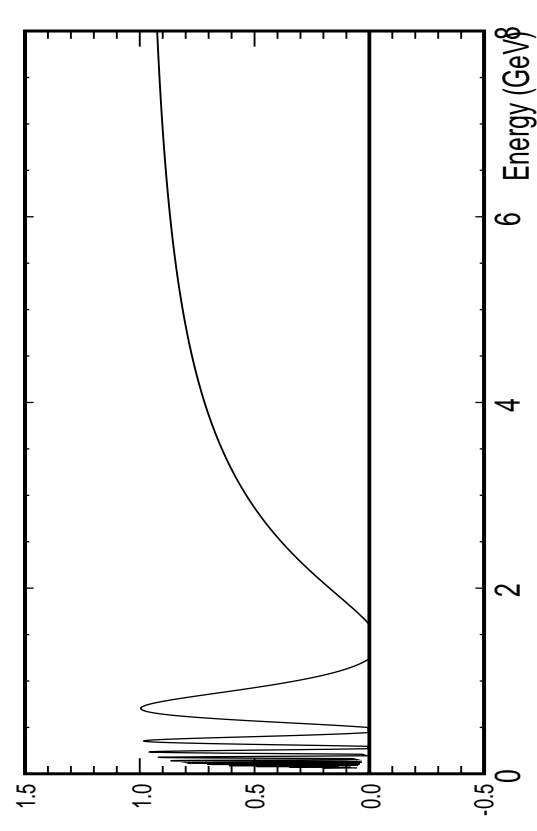

jen!n!uns ${ }^{\mathrm{n}} \mathrm{A}$

¿ 巳

䑻

.

$\sum \stackrel{0}{\Sigma}$

о $\cong$

ปै

类

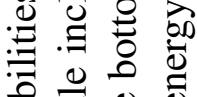

宅 웡

。

ป ป

อ :

氜 氕

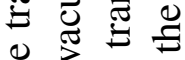

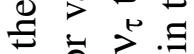

$\underset{4}{0} \uparrow . \overline{0}$

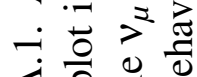

4 일

융 के

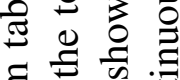

ํㅗㅇ

$\because$ 过

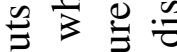

家:

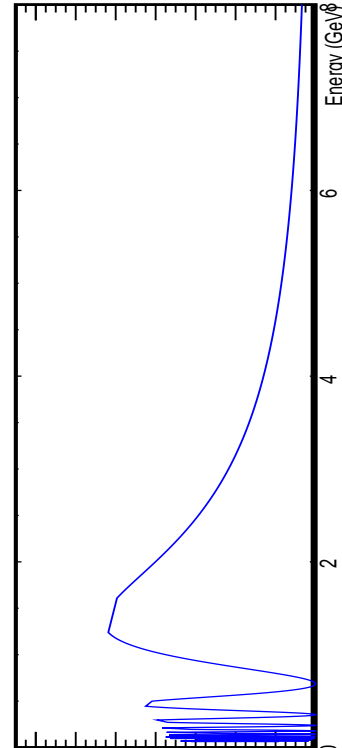

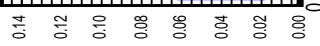
$\left({ }^{\ominus} \wedge \leftarrow{ }^{n} \Lambda\right) d$

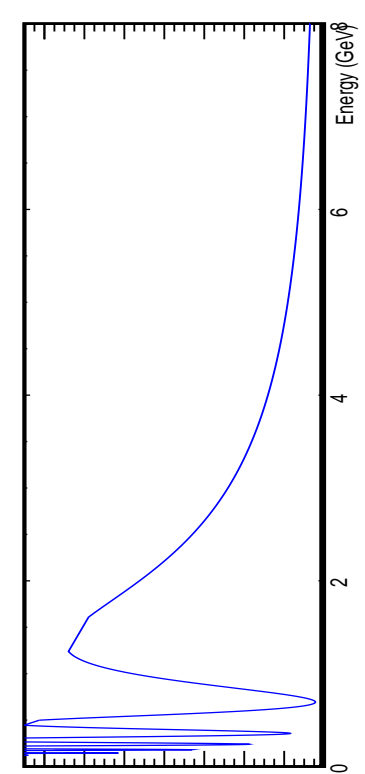

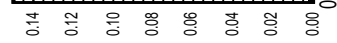
$\left({ }^{\ominus} \wedge \leftarrow{ }^{n} \wedge\right) d$

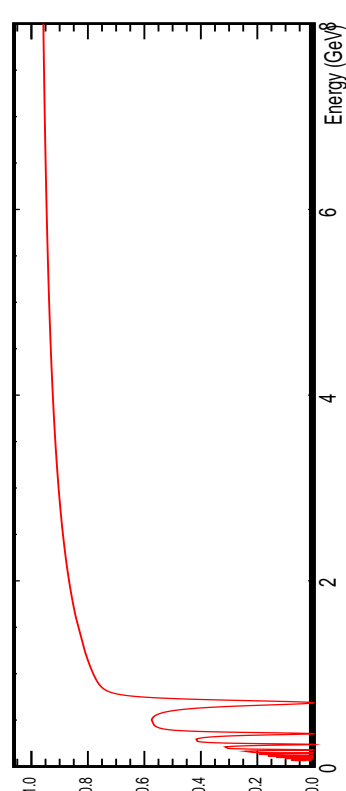

(MSW/Unnoen) o!̣ey
: $\cong$

它 0 2 $\Xi 2$ 范 อ $>\overrightarrow{0}$ 氜

党空

తิ 일 छ

을

ฮี क

ज्ञ

킹

$\exists$ 해유

官江击

竞方

잉

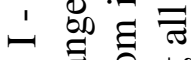

..

远导

콣

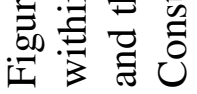




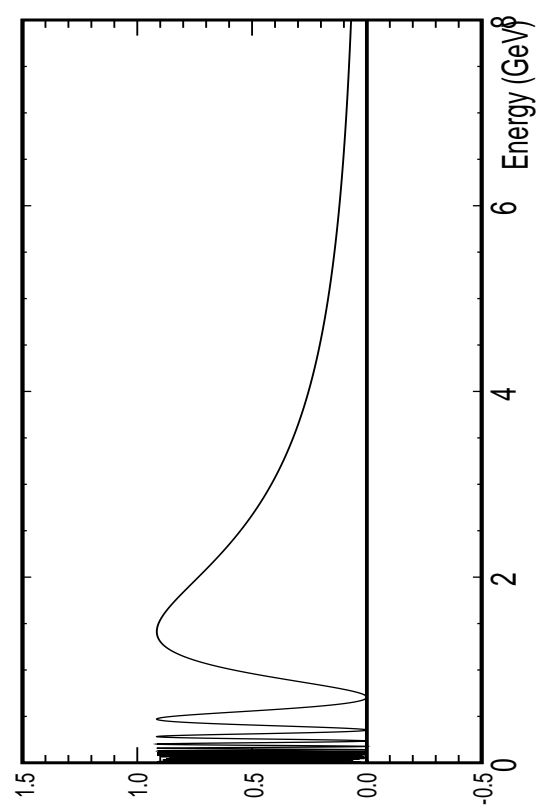

Uo!!! SUEג? ${ }^{2} \wedge$

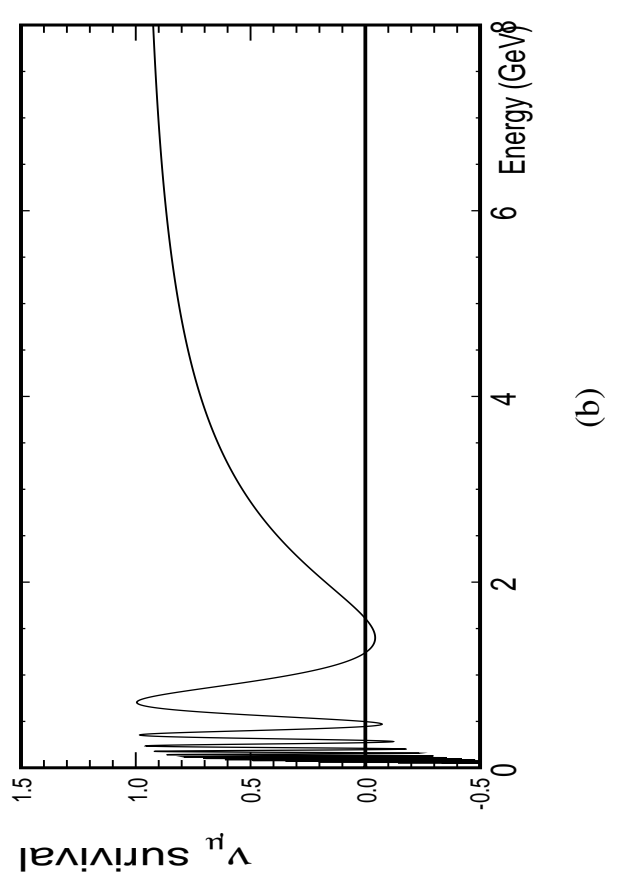

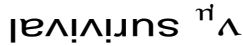

$\stackrel{0}{\stackrel{0}{0} \varangle}$

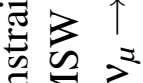

¿ $\sum_{0}^{2}$

일

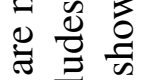

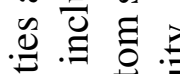

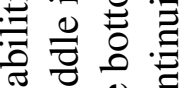

융.

ㅎ․

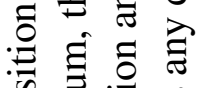

䒕 卷

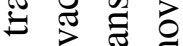
巳

运学

园을 $>$

$<$ 定:

讨

ㅎํㅇำ

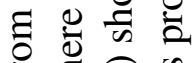

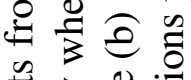

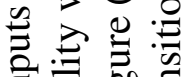

.클

융. $\frac{\pi}{\pi}$

的

둔

ᄒै $\uparrow$ के द्व

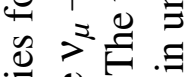

:

ज㱐

త

는

‡Ð

:

:

江。号

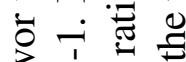

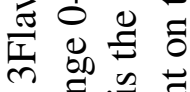

플.

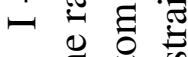

ن롱

¿ $\Xi 80$

롱

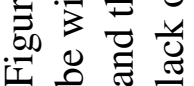




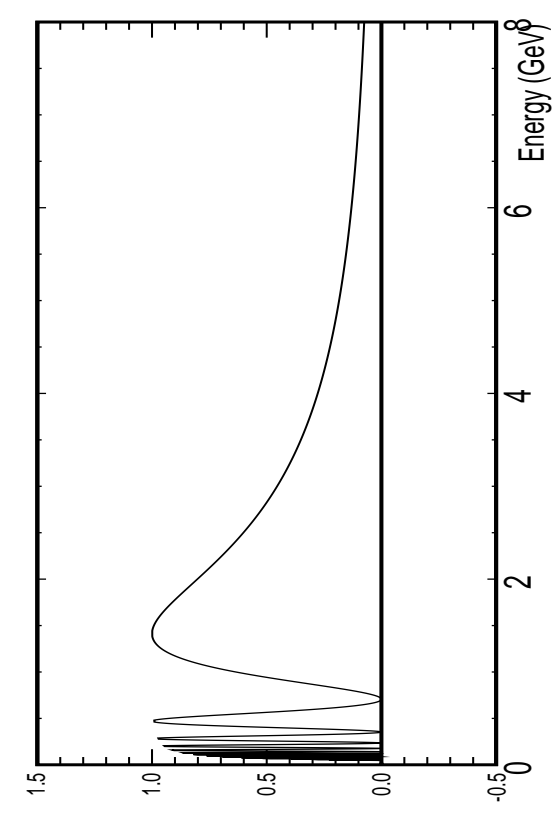

Uо!!!sued? ${ }^{2} \wedge$

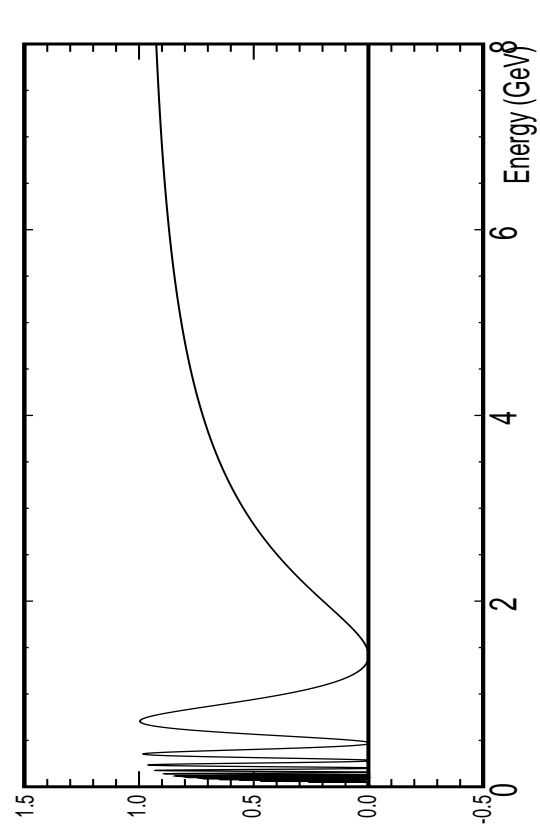

|อง!ก!ıns ${ }^{\mathrm{n}} \wedge$

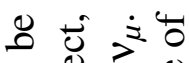

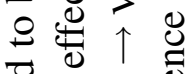
焉 $>^{2}$

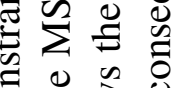
है

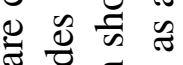
\% $\frac{0}{0} .0$ 氖. 후웡

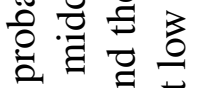
워 : 氜声泀 $\uparrow$ 苛 E

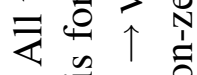

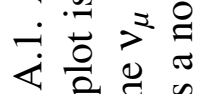
¿ 웜 웡

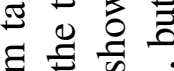
잉 ๑ 0

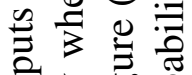
.

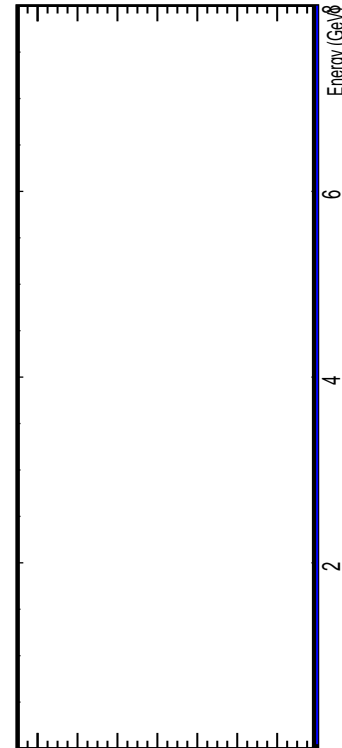

مأسلسلسلسلسلسم: $\left({ }^{\ominus} \wedge \leftarrow{ }^{n} \wedge\right) d$

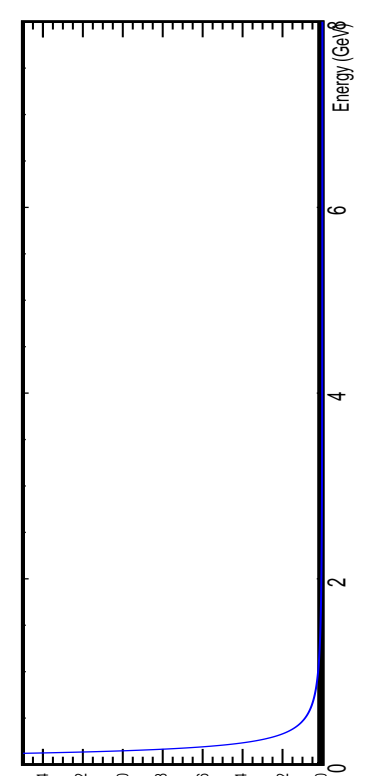

$\left({ }^{a} \wedge \leftarrow{ }^{n} \wedge\right) d$

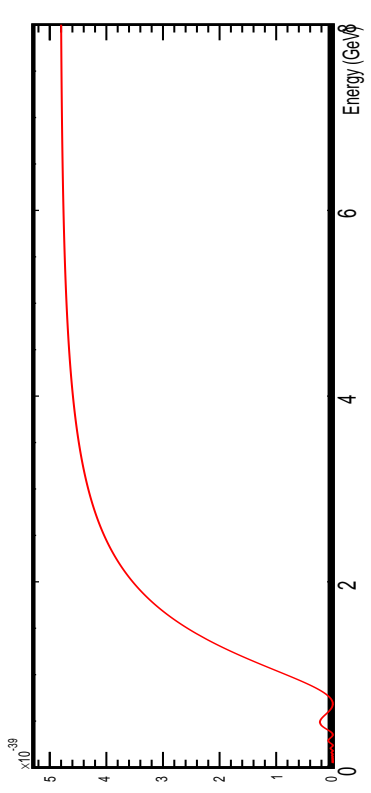

(MSW/unnoen) o!̣ey
四 : 峁 象完:

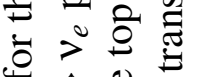
$\underset{0}{0} \uparrow \stackrel{0}{\Xi}$ $:>^{2}: \Xi$

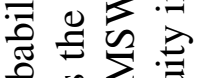
일

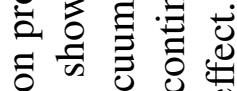
.0ิ ชิ :

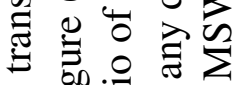

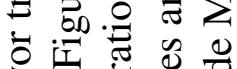

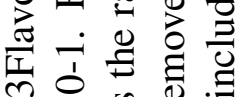
10.0

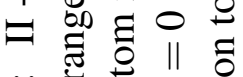
भiष ४ $\doteq 00$

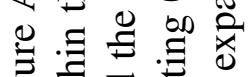

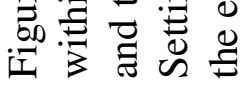




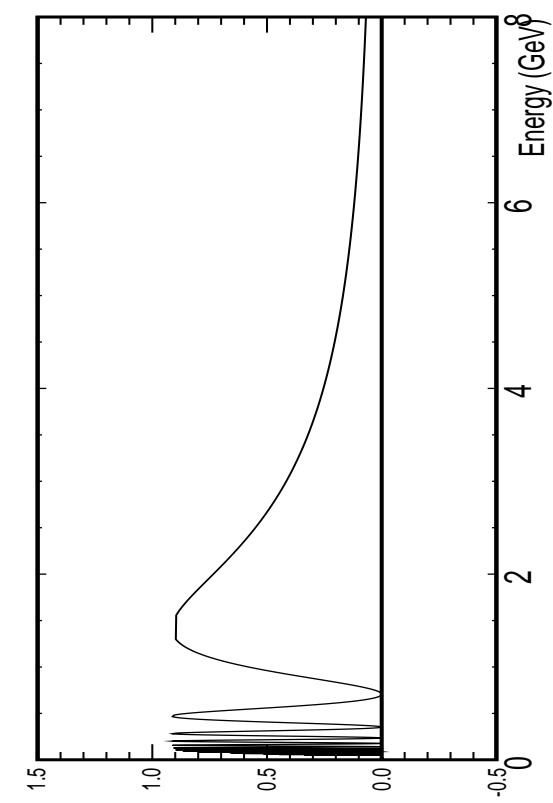

uo!!! SUed? ${ }^{2} \wedge$

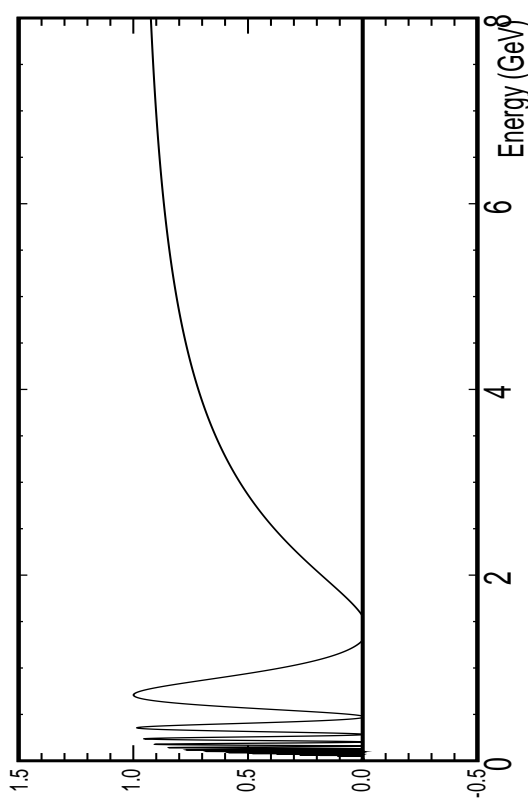

|อง!ก!ıns ${ }^{n} \wedge$

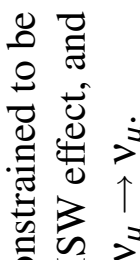
రิ $\sum_{0}^{\infty}$ むै बै :

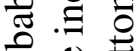
흐웡 ธี่ อ : ฐี 号 元 it 这. $\frac{0}{2} \frac{0}{2}$ ฮิ ڤิ 을 $\stackrel{0}{=} \frac{2}{5}$ .尹 $\frac{0}{3}$

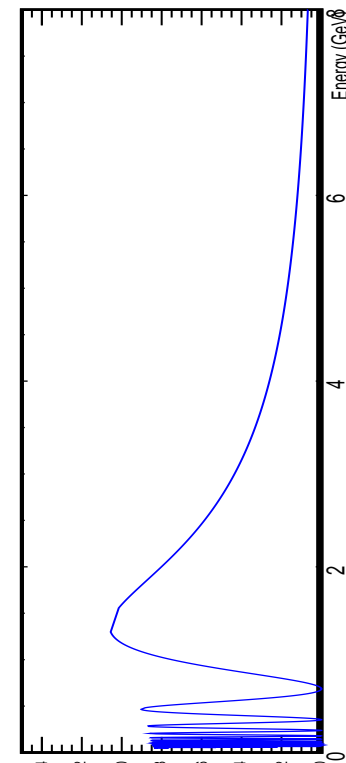

势 $\left({ }^{\ominus} \wedge \leftarrow{ }^{n} \wedge\right) d$

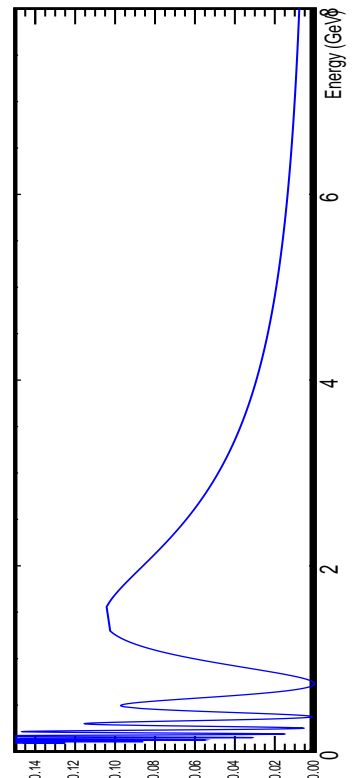

$\left({ }^{\ominus} \wedge \leftarrow{ }^{n} \wedge\right) d$

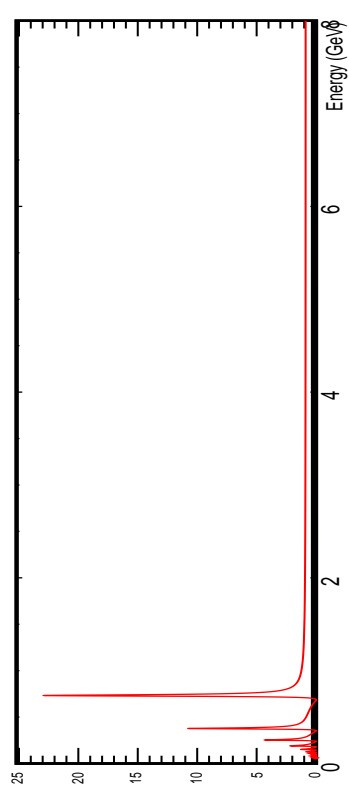

(MSW/unnoen) o!̣ey
三.苛

可

$\nsubseteq \stackrel{\Xi}{0} . \Xi$

흔 흔

.

$>0$

으

త

อั

을

:

(ี)

苛

光理

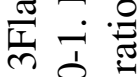

$\stackrel{1}{\exists} 80$

‥ㅈ․

ن்

을

它焉 


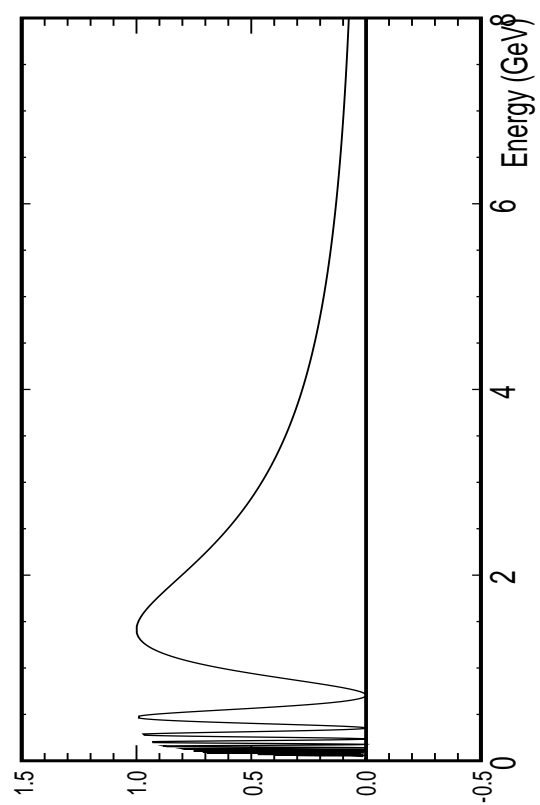

Uo!!!sued? ${ }^{2} \wedge$

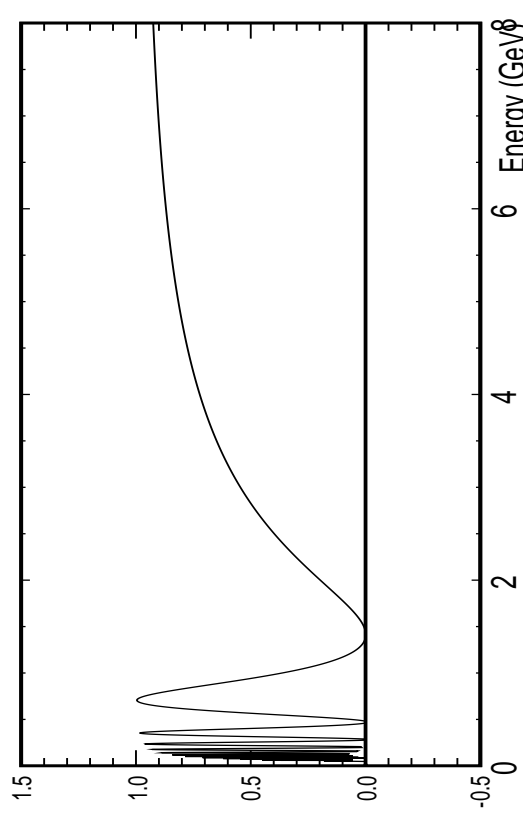

|ел!

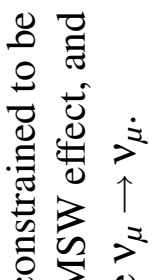

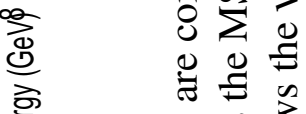

幽造

สิ ฮ

을 亚

드를

อ :

ฐี

孚

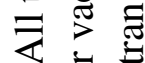

군?

ए।

$\frac{0}{2} \frac{1}{2}$

을

을 吾

哇造

.

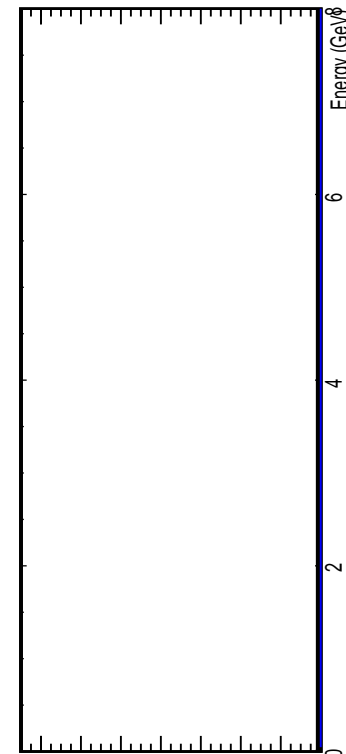

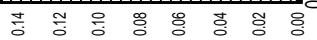
$\left({ }^{\ominus} \wedge \leftarrow{ }^{n} \Lambda\right) d$

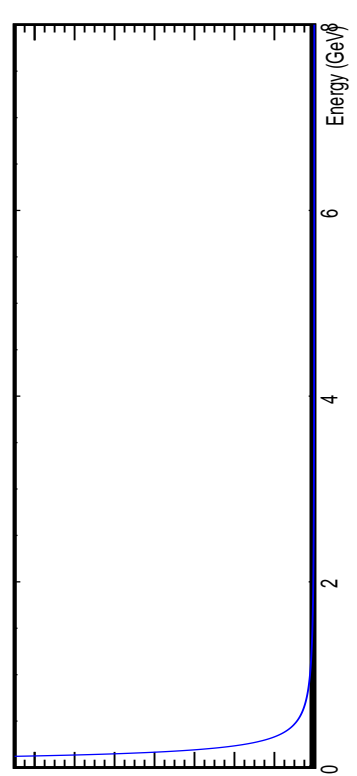

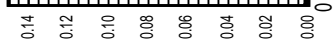
$\left({ }^{\ominus} \wedge \leftarrow{ }^{i n} \wedge\right) d$

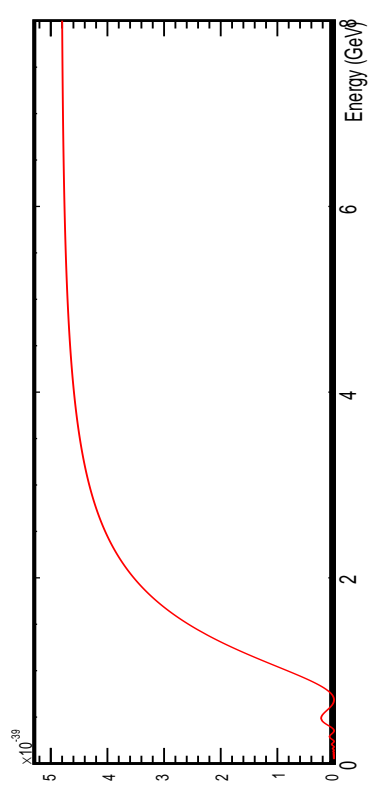

(MSW/unnoes) o!̣ey
之. ․ㅡㄹ

बे

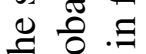

흔 흔

.

$>2$

()

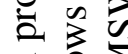

으요

สิ

च

$\exists$

玒

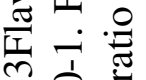

$>80$

$\geq \stackrel{\mathscr{0}}{\tilde{\sigma}}$

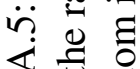

․ㅡㅇ

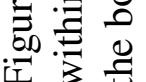




\section{Appendix B}

\section{F/N Systematic Effects}

This section contains the plots of the F/N prediction when a $\pm 1 \sigma$ shift in a systematic error is applied to Far Detector Monte Carlo. The left plot shows the energy spectrum, while the right plot shows the ratio of the predicted/nominal spectrum. The exposure is $2.5 \times 10^{20}$ POT. The F/ $\mathrm{N}$ methods robustness to energy dependent systematics is highlighted by the CCMA uncertainty.

Fig. B.3 shows that the CCMA uncertainty causes a large shift in the energy spectrum at low energies. The magnitude of the shift in the energy spectrum does not translate to a sizeable shift in the oscillation parameters. The average shifts taken from Table 6.2 show that the uncertainy on $\Delta \mathrm{m}_{31}^{2}$ is ${ }_{-0.0109}^{+0.0113}$ and $\theta_{13}$ is ${ }_{-0.00989616}^{+0.000314163}$. The limited affect on the oscillation parameters is due to the CCMA energy dependent effect dividing out in the F/N Monte Carlo ratio. 

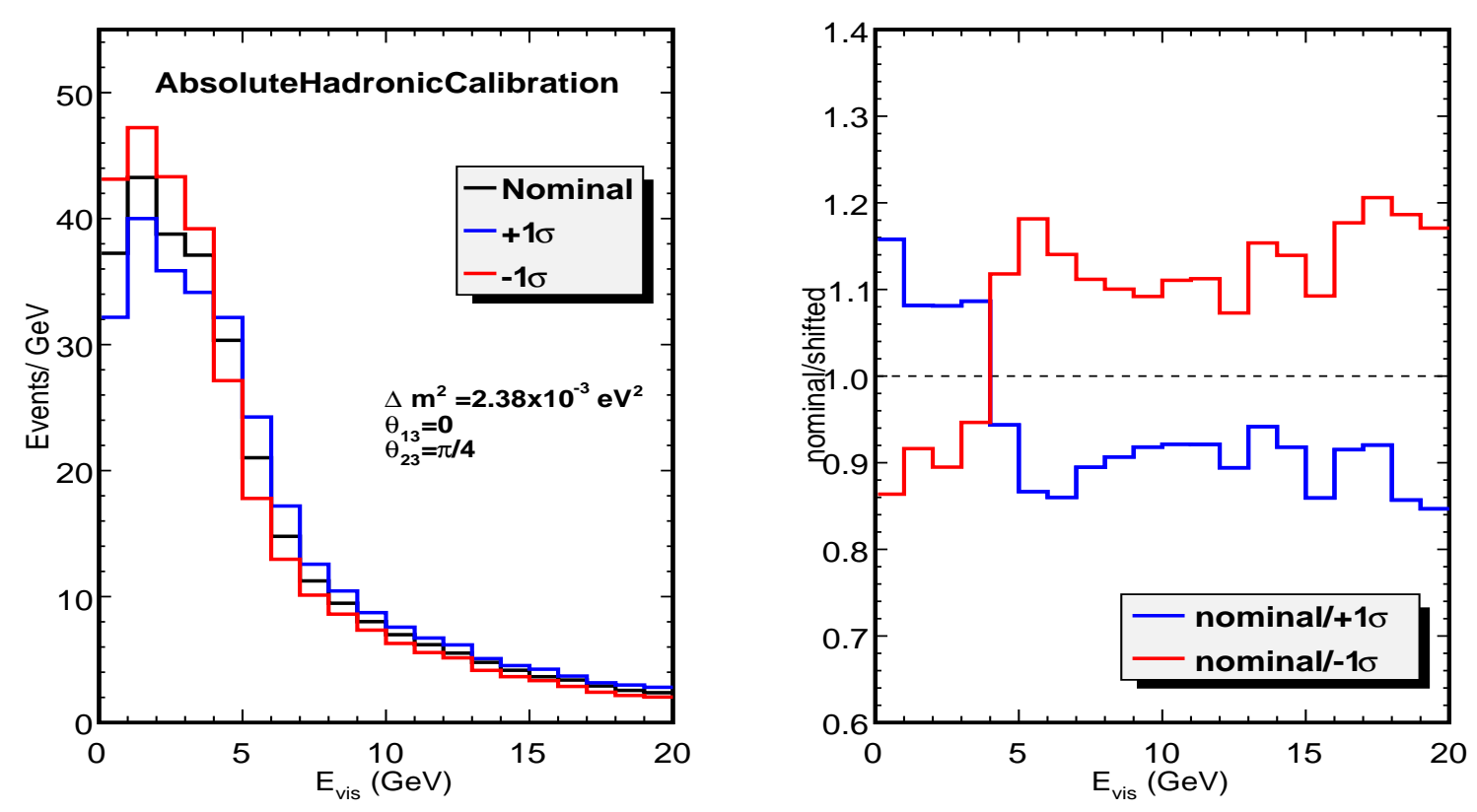

Figure B.1: Energy spectrum shift caused by the changing the absolute hadronic calibration uncertainty by $\pm 1 \sigma$.
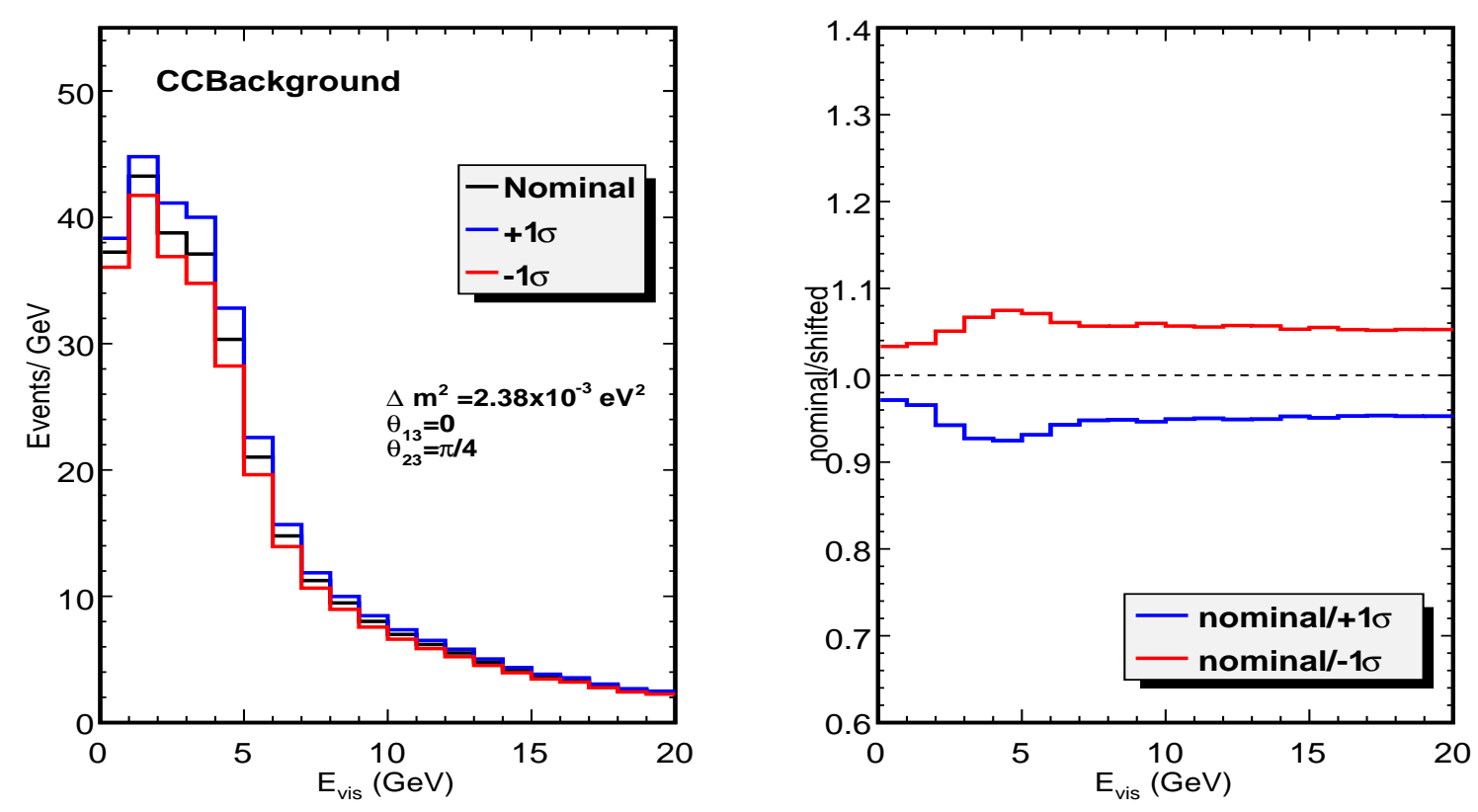

Figure B.2: Energy spectrum shift caused by the changing the $\mathrm{CC}$ background uncertainty by $\pm 1 \sigma$. 

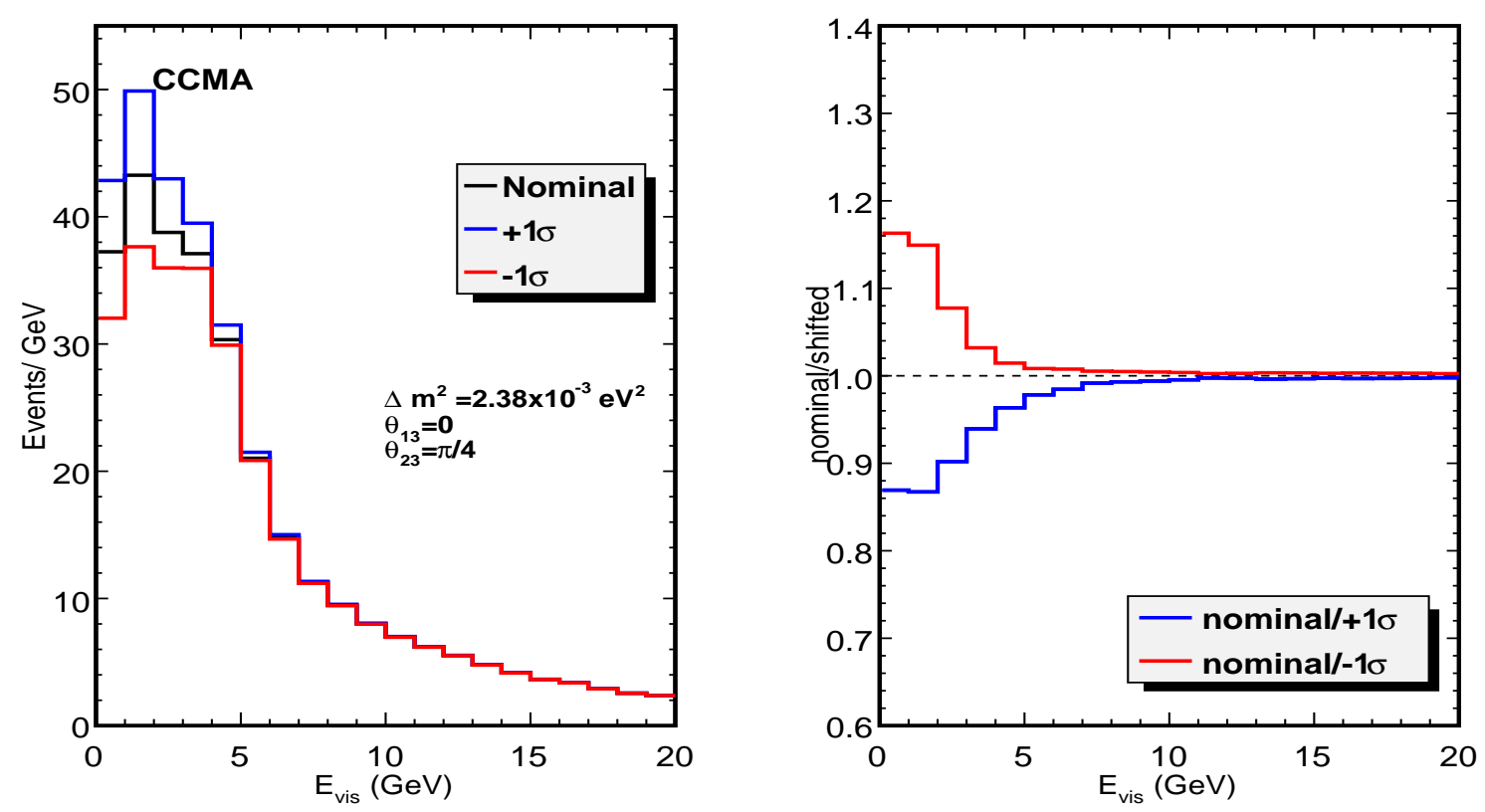

Figure B.3: Energy spectrum shift caused by the changing the CCMA uncertainty by $\pm 1 \sigma$.
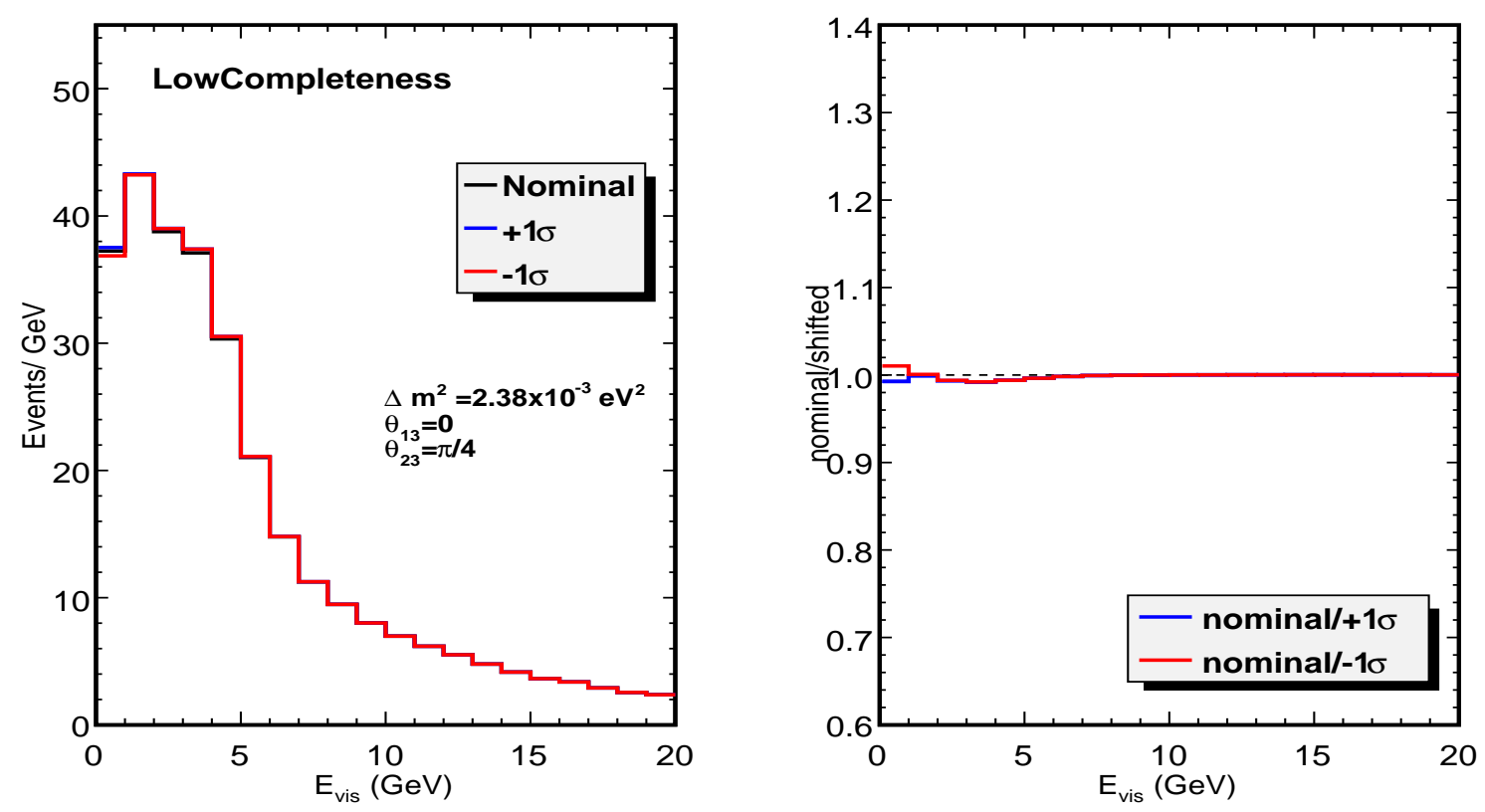

Figure B.4: Energy spectrum shift caused by the changing the uncertainty on the low completeness by $\pm 1 \sigma$. 

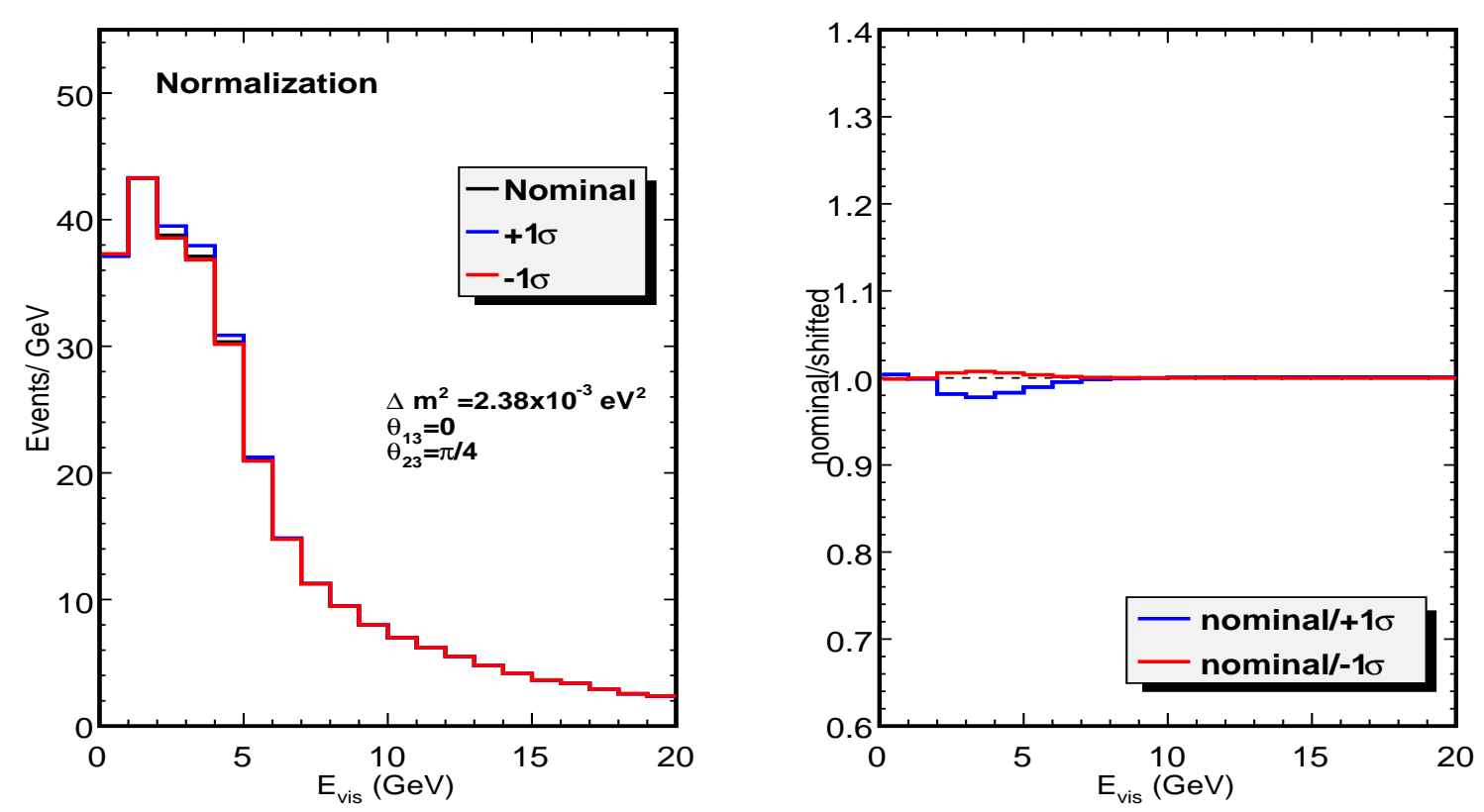

Figure B.5: Energy spectrum shift caused by the changing the normalization uncertainty by $\pm 1 \sigma$.
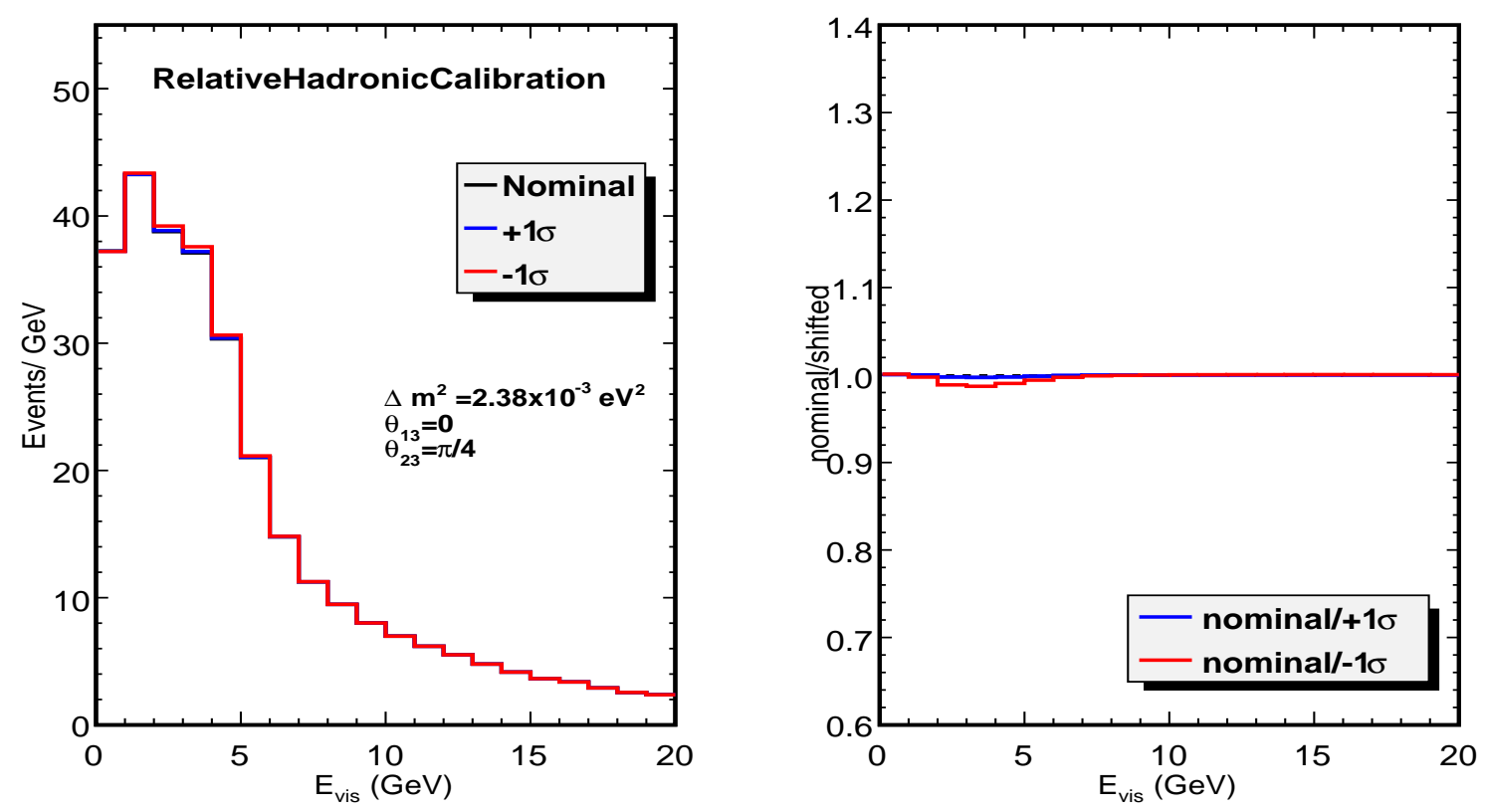

Figure B.6: Energy spectrum shift caused by the changing the relative hadronic calibration uncertainty by $\pm 1 \sigma$. 

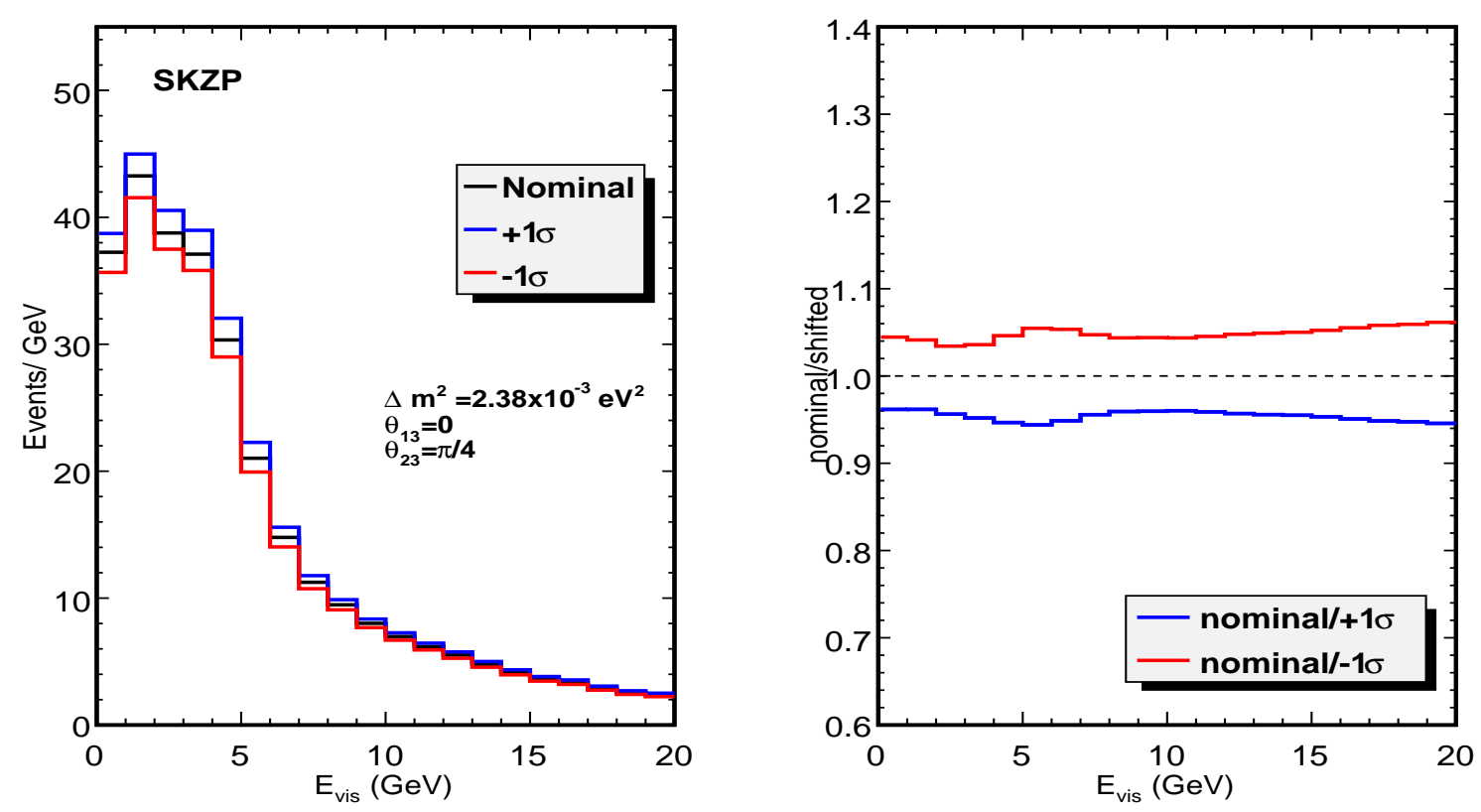

Figure B.7: Energy spectrum shift caused by the changing the uncertainty SKZP beam reweighting by $\pm 1 \sigma$. 


\section{Bibliography}

[1] W. Pauli. Dear radioactive ladies and gentlemen. Phys. Today, 31N9:27, 1978.

[2] F. Reines and C. L. Cowan. A proposed experiment to detect the free neutrino. Phys. Rev., 90:492-493, 1953.

[3] F. Reines and C. L. Cowan. Detection of the free neutrino. Phys. Rev., 92:830$831,1953$.

[4] C. L. Cowan, F. Reines, F. B. Harrison, H. W. Kruse, and A. D. McGuire. Detection of the free neutrino: A Confirmation. Science, 124:103-104, 1956.

[5] Jr. Davis, Raymond, Don S. Harmer, and Kenneth C. Hoffman. Search for neutrinos from the sun. Phys. Rev. Lett., 20:1205-1209, 1968.

[6] P. Anselmann et al. Implications of the GALLEX determination of the solar neutrino flux. Phys. Lett., B285:390-397, 1992.

[7] Dzh. N. Abdurashitov et al. The Russian-American gallium experiment (SAGE) Cr neutrino source measurement. Phys. Rev. Lett., 77:4708-4711, 1996.

[8] R. Becker-Szendy et al. A Search for muon-neutrino oscillations with the IMB detector. Phys. Rev. Lett., 69:1010-1013, 1992. 
[9] M. Ambrosio et al. Measurement of the atmospheric neutrino-induced upgoing muon flux using MACRO. Phys. Lett., B434:451-457, 1998, hepex/9807005.

[10] M. Ambrosio et al. Atmospheric neutrino oscillations from upward throughgoing muon multiple scattering in MACRO. Phys. Lett., B566:35-44, 2003, hep-ex/0304037.

[11] W. W. M. Allison et al. The atmospheric neutrino flavor ratio from a 3.9 fiducial kiloton-year exposure of Soudan 2. Phys. Lett., B449:137-144, 1999, hep-ex/9901024.

[12] M. Sanchez et al. Observation of atmospheric neutrino oscillations in soudan 2. Phys. Rev., D68:113004, 2003, hep-ex/0307069.

[13] K. S. Hirata et al. Observation of a small atmospheric $v_{\mu} / v_{e}$ ratio in Kamiokande. Phys. Lett., B280:146-152, 1992.

[14] B. Pontecorvo. Inverse beta processes and nonconservation of lepton charge. Sov. Phys. JETP, 7:172-173, 1958.

[15] B. Pontecorvo. Neutrino experiments and the question of leptonic-charge conservation. Sov. Phys. JETP, 26:984-988, 1968.

[16] Z. Maki, M. Nakagawa, and S. Sakata. Remarks on the unified model of elementary particles. Prog. Theor. Phys., 28:870, 1962.

[17] John N. Bahcall and S. C. Frautschi. Lepton non-conservation and solar neutrinos. Phys. Lett., B29:623-625, 1969.

[18] Q. R. Ahmad et al. Measurement of the charged current interactions produced by b-8 solar neutrinos at the sudbury neutrino observatory. Phys. Rev. Lett., 87:071301, 2001, nucl-ex/0106015. 
[19] S. N. Ahmed et al. Measurement of the total active B-8 solar neutrino flux at the Sudbury Neutrino Observatory with enhanced neutral current sensitivity. Phys. Rev. Lett., 92:181301, 2004, nucl-ex/0309004.

[20] Y. Fukuda et al. Evidence for oscillation of atmospheric neutrinos. Phys. Rev. Lett., 81:1562-1567, 1998, hep-ex/9807003.

[21] J. Fetter, G. C. McLaughlin, A. B. Balantekin, and G. M. Fuller. Active-sterile neutrino conversion: Consequences for the r-process and supernova neutrino detection. Astropart. Phys., 18:433-448, 2003, hep-ph/0205029.

[22] C. Athanassopoulos et al. Evidence for anti-nu/mu $\rightarrow$ anti-nu/e oscillation from the LSND experiment at the Los Alamos Meson Physics Facility. Phys. Rev. Lett., 77:3082-3085, 1996, nucl-ex/9605003.

[23] A. A. Aguilar-Arevalo et al. A Search for electron neutrino appearance at the $\Delta m^{2} \sim 1 \mathrm{eV}^{2}$ scale. Phys. Rev. Lett., 98:231801, 2007, 0704.1500.

[24] A. A. Aguilar-Arevalo et al. A Search for Electron Antineutrino Appearance at the $\Delta m^{2} \sim 1 \mathrm{eV}^{2}$ Scale. 2009, 0904.1958.

[25] Alexis A. Aguilar-Arevalo et al. A search for muon neutrino and antineutrino disappearance in MiniBooNE. 2009, 0903.2465.

[26] Michele Maltoni and Thomas Schwetz. Sterile neutrino oscillations after first MiniBooNE results. Phys. Rev., D76:093005, 2007, 0705.0107.

[27] Q. R. Ahmad et al. Direct evidence for neutrino flavor transformation from neutral-current interactions in the sudbury neutrino observatory. Phys. Rev. Lett., 89:011301, 2002, nucl-ex/0204008. 
[28] M. Maltoni, T. Schwetz, M. A. Tortola, and J. W. F. Valle. Constraining neutrino oscillation parameters with current solar and atmospheric data. Phys. Rev., D67:013011, 2003, hep-ph/0207227.

[29] Abhijit Bandyopadhyay, Sandhya Choubey, Srubabati Goswami, and D. P. Roy. Implications of the first neutral current data from SNO for solar neutrino oscillation. Phys. Lett., B540:14-19, 2002, hep-ph/0204286.

[30] S. Fukuda et al. Tau neutrinos favored over sterile neutrinos in atmospheric muon neutrino oscillations. Phys. Rev. Lett., 85:3999-4003, 2000, hepex/0009001.

[31] K. Abe et al. A measurement of atmospheric neutrino flux consistent with tau neutrino appearance. Phys. Rev. Lett., 97:171801, 2006, hep-ex/0607059.

[32] K. Kodama et al. Observation of tau-neutrino interactions. Phys. Lett., B504:218-224, 2001, hep-ex/0012035.

[33] A. Leike, T. Riemann, and J. Rose. S matrix approach to the Z line shape. Phys. Lett., B273:513-518, 1991, hep-ph/9508390.

[34] Precision electroweak measurements on the $\mathrm{Z}$ resonance. Phys. Rept., 427:257, 2006, hep-ex/0509008.

[35] C. Amsler et al. Review of particle physics. Phys. Lett., B667:1, 2008.

[36] P. Adamson et al. Measurement of Neutrino Oscillations with the MINOS Detectors in the NuMI Beam. Phys. Rev. Lett., 101:131802, 2008, 0806.2237.

[37] Yuichi Oyama. K2k (kek to kamioka) neutrino oscillation experiment at kekps. 1998, hep-ex/9803014.

[38] D. Duchesneau. The cern - gran sasso neutrino program. eConf, C0209101:TH09, 2002, hep-ex/0209082. 
[39] N. Cabibbo. Unitary Symmetry and Leptonic Decays. Phys. Rev. Lett., 10:531-532, 1963.

[40] Makoto Kobayashi and Toshihide Maskawa. CP Violation in the Renormalizable Theory of Weak Interaction. Prog. Theor. Phys., 49:652-657, 1973.

[41] Boris Kayser. Neutrino mass, mixing, and flavor change. 2002, hep$\mathrm{ph} / 0211134$.

[42] L. Wolfenstein. Neutrino oscillations in matter. Phys. Rev., D17:2369, 1978.

[43] S. P. Mikheev and A. Yu. Smirnov. Resonant amplification of neutrino oscillations in matter and solar neutrino spectroscopy. Nuovo Cim., C9:17-26, 1986.

[44] S. P. Mikheev and A. Yu. Smirnov. Resonance enhancement of oscillations in matter and solar neutrino spectroscopy. Sov. J. Nucl. Phys., 42:913-917, 1985.

[45] A. Cervera et al. Golden measurements at a neutrino factory. Nucl. Phys., B579:17-55, 2000, hep-ph/0002108.

[46] Osamu Yasuda. Three flavor neutrino oscillation analysis of the Superkamiokande atmospheric neutrino data. 1998, hep-ph/9809205.

[47] M. Apollonio et al. Search for neutrino oscillations on a long base-line at the CHOOZ nuclear power station. Eur. Phys. J., C27:331-374, 2003, hepex/0301017.

[48] S. Abe et al. Precision Measurement of Neutrino Oscillation Parameters with KamLAND. Phys. Rev. Lett., 100:221803, 2008, 0801.4589.

[49] A. J. T. Holmes. Negative hydrogen ion beams. Plasma Physics and Controlled Fusion, 34:653-676, May 1992. 
[50] Charles W. Schmidt. Review of negative hydrogen ion sources. 1990. Presented at 1990 Linear Accelerator Conf., Albuquerque, NM, Sep 10-14, 1990.

[51] M. G. Catanesi et al. Measurement of the production cross-section of positive pions in $\mathrm{p} \mathrm{Al} \mathrm{collisions} \mathrm{at} \mathrm{12.9-GeV/c.} \mathrm{Nucl.} \mathrm{Phys.,} \mathrm{B732:1-45,} \mathrm{2006,} \mathrm{hep-}$ ex/0510039.

[52] J. G. Asbury et al. Pion production at 12 degrees and 15 degrees in protonberyllium collisions at 12.5 gev/c. Phys. Rev., 178:2086-2088, 1969.

[53] T. Alber et al. Charged particle production in proton, deuteron, oxygen and sulphur nucleus collisions at 200-GeV per nucleon. Eur. Phys. J., C2:643659, 1998, hep-ex/9711001.

[54] G. Ambrosini et al. Measurement of charged particle production from 450GeV/c protons on beryllium. Eur. Phys. J., C10:605-627, 1999.

[55] Sacha E. Kopp. Accelerator neutrino beams. Phys. Rept., 439:101-159, 2007, physics/0609129.

[56] R. A. Lundy, T. B. Novey, D. D. Yovanovitch, and V. L. Telegdi. pi + /- and K + /- Production Cross Sections for 12.5-BeV Protons on Be. Phys. Rev. Lett., 14:504-507, 1965.

[57] G. J. Marmer et al. Differential production cross-sections of low-momentum particles from 12.3-bev/c protons on beryllium and copper. Phys. Rev., 179:1294-1300, 1969.

[58] M. Bonesini, A. Marchionni, F. Pietropaolo, and T. Tabarelli de Fatis. On particle production for high energy neutrino beams. Eur. Phys. J., C20:1327, 2001, hep-ph/0101163. 
[59] F. Ballarini et al. The FLUKA code: An overview. J. Phys. Conf. Ser., 41:151-160, 2006.

[60] S. Sacha et al. Beam flux position paper. minos-doc 1278, MINOS, December 2005 .

[61] S. Kopp et al. Using gnumi ntuple for muon monitor rates. minos-doc 2449, MINOS, November 2006.

[62] S. Kopp et al. Gnumi weights for muon monitors. minos-doc 2539, MINOS, December 2006.

[63] S. Kopp et al. Physical composition of the numi and g4numi monte carlo hadron absorber and muon monitor alcoves. minos-doc 2769, MINOS, March 2007.

[64] D. G. Michael. The magnetized steel and scintillator calorimeters of the MINOS experiment. 2008, 0805.3170.

[65] R5900-00-M16, http://sales.hamamatsu.com/index.php?id=13189446.

[66] multianode photomultiplier tube R5900-00-M64, january 1999, http://sales.hamamatsu.com/assets/pdf/parts_R/R590000M64.pdf.

[67] P. Adamson et al. The MINOS light injection calibration system. Nucl. Instrum. Meth., A492:325-343, 2002, hep-ex/0204021.

[68] D.J. Koskinen. An overview of the main injector neutrino oscillation search, the rack protection system and method to degauss a large iron calorimeter particle detector. Master's thesis, University of Minnesota Duluth, December 2004.

[69] B. Rebel. Neutrino-Induced Muons in the MINOS Far Detector. PhD thesis, Indiana University, August 2004. 
[70] P. Adamson et al. First observations of separated atmospheric nu/mu and anti-nu/mu events in the MINOS detector. Phys. Rev., D73:072002, 2006, hep-ex/0512036.

[71] P. Adamson et al. A Study of Muon Neutrino Disappearance Using the Fermilab Main Injector Neutrino Beam. Phys. Rev., D77:072002, 2008, 0711.0769.

[72] Gian Luigi Fogli, E. Lisi, and A. Marrone. Four-neutrino oscillation solutions of the atmospheric neutrino anomaly. Phys. Rev., D63:053008, 2001, hep$\mathrm{ph} / 0009299$.

[73] Michel Sorel, Janet M. Conrad, and Michael Shaevitz. A combined analysis of short-baseline neutrino experiments in the $(3+1)$ and $(3+2)$ sterile neutrino oscillation hypotheses. Phys. Rev., D70:073004, 2004, hep-ph/0305255.

[74] Phillip Litchfield. Far detector event preselection (data cleaning) for nc analysis. minos-doc 3377, MINOS, July 2007.

[75] S. Avvakumov et al. Spontaneous light emission from fibers in MINOS. Nucl. Instrum. Meth., A545:145-155, 2005.

[76] T. Osiecki et al. Cuts based neutral current selection for the minos near detector. minos-doc 2589, MINOS, Februrary 2006.

[77] K. Lang and R. Ospanov. A muon identification technique using the k-nearest neighbor algorithm. minos-doc 2737, MINOS, April 2008.

[78] A. Sousa. Nc/cc separation using multivariate discriminant analysis. minosdoc 2794, MINOS, March 2007.

[79] R. Pittam. Ann based neutral current extraction method. minos-doc 3130, MINOS, June 2007. 
[80] R. Pittam and A. Sousa. Pid selection for use in the nc analysis. minos-doc 3907, MINOS, September 2007.

[81] T. Osiecki. A search for sterile neutrinos in MINOS. PhD thesis, The University of Texas at Austin, December 2007.

[82] F. James and M. Roos. Minuit: A System for Function Minimization and Analysis of the Parameter Errors and Correlations. Comput. Phys. Commun., 10:343-367, 1975 .

[83] H. Gallagher. The NEUGEN neutrino event generator. Nucl. Phys. Proc. Suppl., 112:188-194, 2002.

[84] Michael Kordosky. Ramifications of intranuclear re-scattering in MINOS. Nucl. Phys. Proc. Suppl., 159:223-228, 2006, hep-ex/0602029.

[85] Y. Itow et al. The JHF-Kamioka neutrino project. 2001, hep-ex/0106019.

[86] D. S. Ayres et al. NOvA proposal to build a 30-kiloton off-axis detector to study neutrino oscillations in the Fermilab NuMI beamline. 2004, hepex/0503053.

[87] Mayly C. Sanchez. Electron neutrino appearance in the MINOS experiment. AIP Conf. Proc., 981:148-150, 2008.

[88] Alessandro Strumia and Francesco Vissani. Neutrino masses and mixings and. 2006, hep-ph/0606054. 